Document ID:

Revision ID:

\title{
PROCUREMENT SPECIFICATION
}

\section{Three Mile Island-II Back Up Heated VAC Dryer System (TMI-2 Fuel Vacuum Dryer)}


412.15

$02 / 21 / 2000$

Rev. 02
DOCUMENT MANAGEMENT CONTROL SYSTEM (DMCS)

DOCUMENT APPROVAL SHEET
Document Type : PROCUREMENT SPECIFICATION

Title : Three Mile Island-II Back Up Heated VAC Dryer System

Author: Nicholas Z. Hertelendy

Document Owner

Michael S. Edgett
Document Identifier :

SPC-287

Project No. (if applicable) :

N/A

Phone :

526-0975

Phone :

$526-3820$

REVIEW CONCURRENCE AND APPROVAL SIGNATURES

Denote $\mathrm{R}$ for review concurrence, $\mathrm{A}$ for approval, as appropriate.

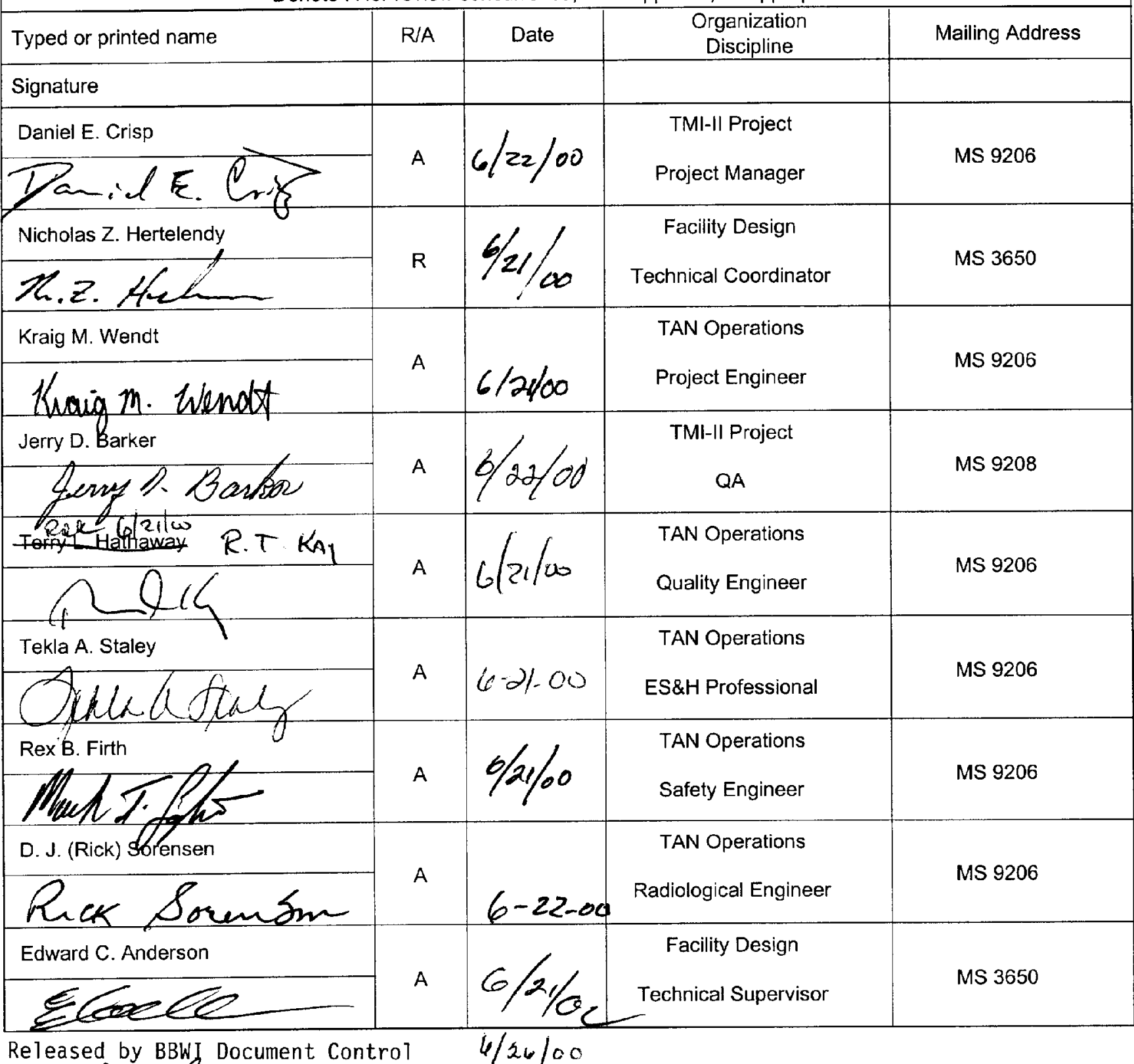

6-1202 Am. Fiah 


\section{TMI-2 Fuel Vacuum Dryer Specification}

The following Procurement Specification was prepared under the responsible charge of the Professional Engineer as indicated by the seal and signature below.

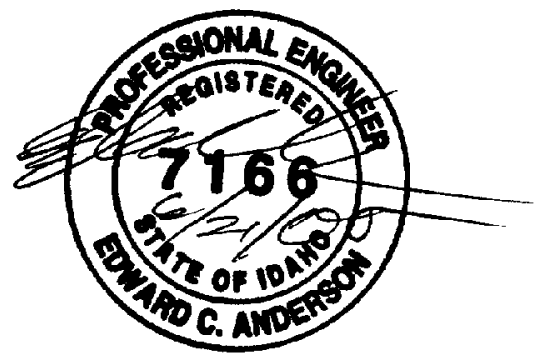


Project Title:

Document Type:

SPC: 287
TMI-2 FUEL VACUUM DRYER SPECIFICATION

Procurement Specification Project Number: N/A

$1.0 \quad$ SCOPE

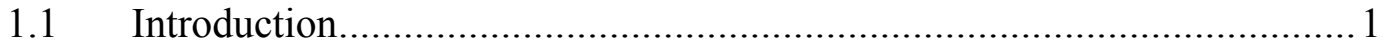

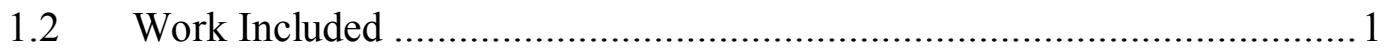

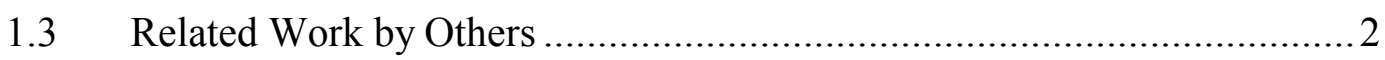

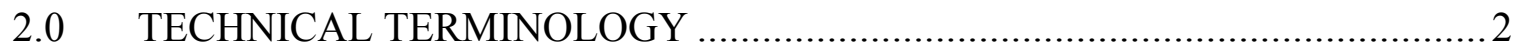

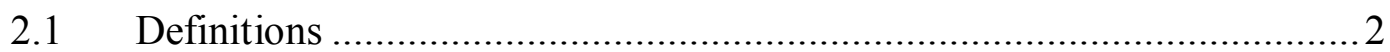

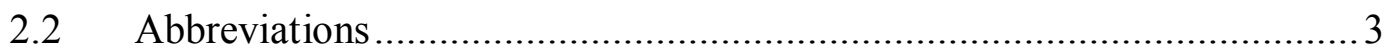

3.0 APPLICABLE CODES, STANDARDS, AND REFERENCES ..........................

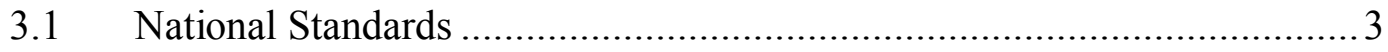

3.2 TMI-2 Canister Heated Vacuum Drying System HVDS Drawing List......5

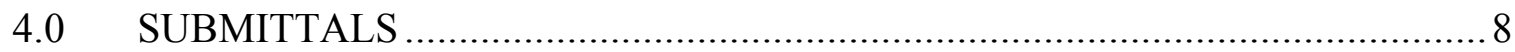

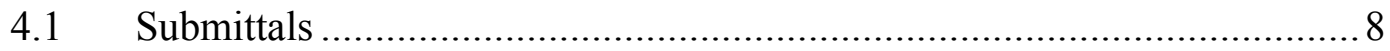

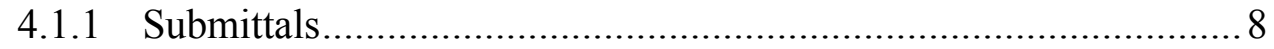

4.1.2 Certificate of Compliance ....................................................... 10

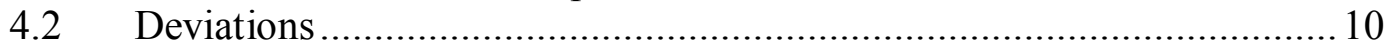

$4.3 \quad$ Release to Ship.............................................................................. 10

5.0 DESIGN

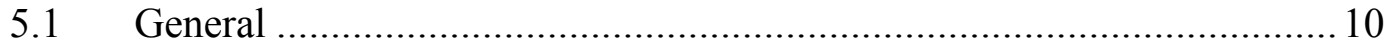

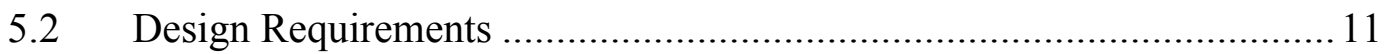

5.2.1 General System Requirements ................................................. 11

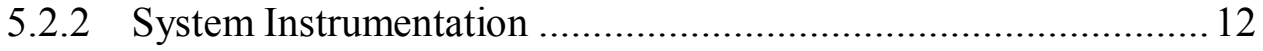

5.2.3 Vacuum Furnace Assembly ........................................................ 13

5.2.4 Condenser Assembly ............................................................ 14

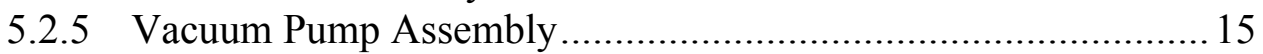

5.2.6 Hose Assemblies and Fittings ……………………………........15

5.2.7 Vacuum Pump and Vacuum Condenser Skid Assemblies............. 16

6.0 ENVIRONMENTAL AND SERVICE CONDITIONS ……............................ 16

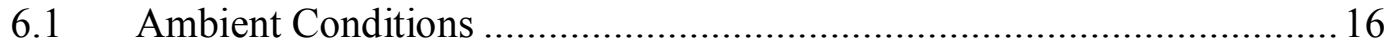

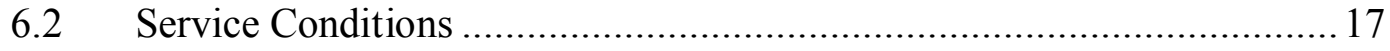

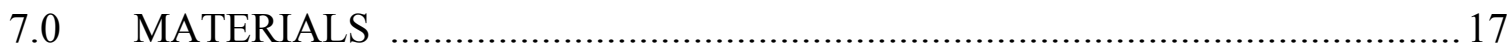


Project Title:

TMI-2 FUEL VACUUM DRYER SPECIFICATION

Document Type:

Procurement Specification

Project Number:

N/A

SPC: 287

8.0 MANUFACTURING/ASSEMBLY

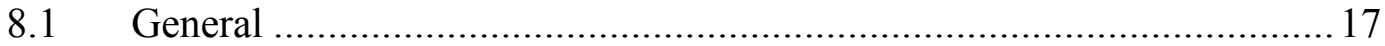

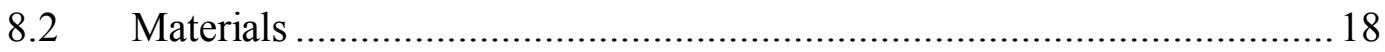

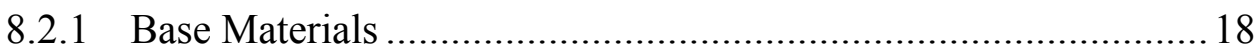

8.2.1.1 Certified Material Test Reports and Certification ............. 18

8.2.2 Welding Material................................................................... 18

8.2.2.1 Welding Filler Metal Certified Material Test Reports......19

8.2.2.2 Welding Material Handling, Storage and Control ............. 19

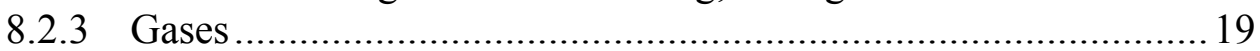

8.2.4 Liquid Penetrant .................................................................. 19

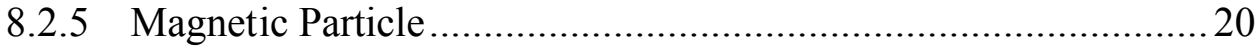

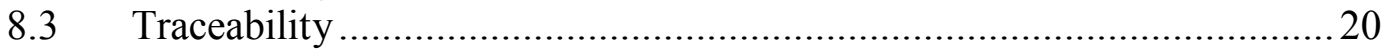

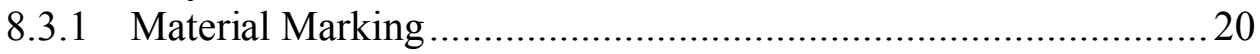

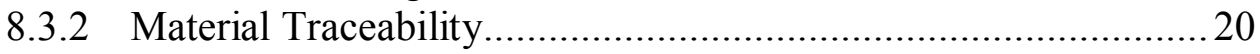

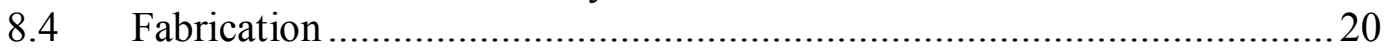

8.4.1 Welding Operations ...............................................................20

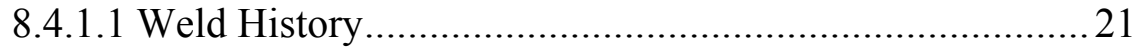

8.4.1.2 Welding Processes .......................................................21

8.4.1.3 Preparation of Base Metal .............................................. 22

8.4.1.4 Welding Requirements ................................................. 22

8.4.1.5 Temporary Welds........................................................22

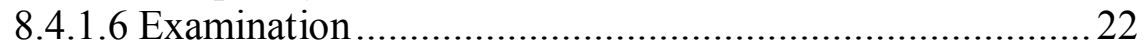

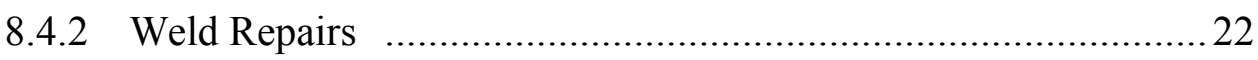

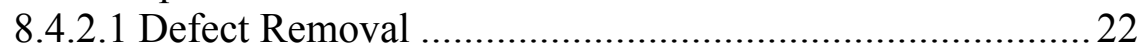

8.4.2.2 Weld Repair Procedures................................................22

8.4.2.3 Weld Repair Examination ..............................................23

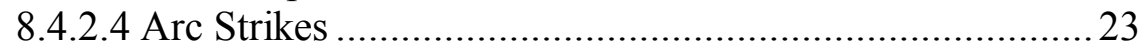

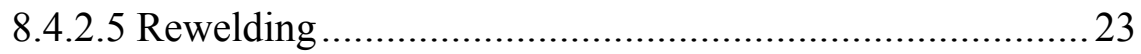

8.4.2.6 Weld Repair Reports ....................................................23

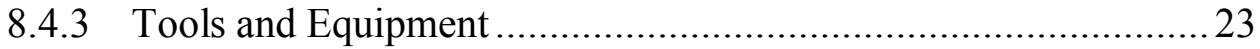

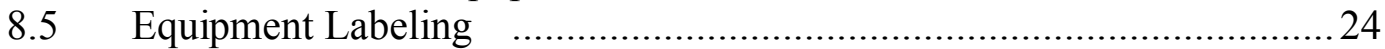

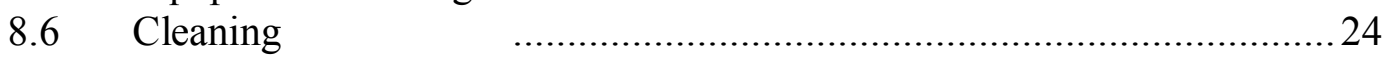

8.6.1 Cleanliness of Piping, Tubing and Vessel .................................2 24

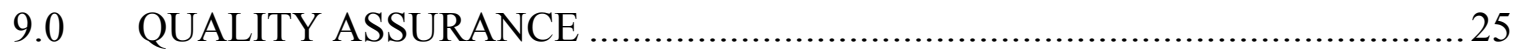

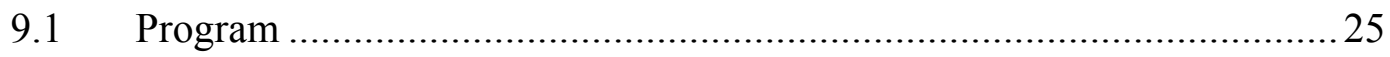

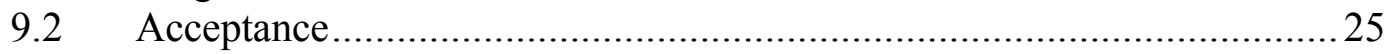

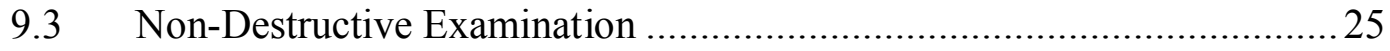

9.3.1 Nondestructive Examination Procedures ………………..............25

9.3.2 Nondestructive Examination Personnel Qualifications ................26

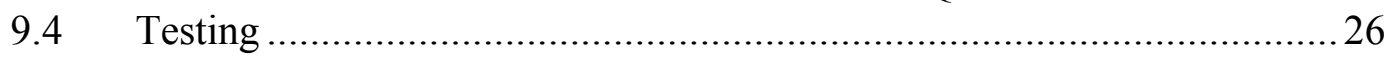

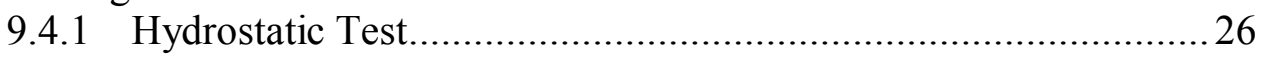


Project Title: $\quad$ TMI-2 FUEL VACUUM DRYER SPECIFICATION

Document Type: Procurement Specification Project Number: N/A

SPC: 287

9.4.2 Leak Test........................................................................... 26

9.4.3 Heater and Thermocouple (TC) Testing .................................22

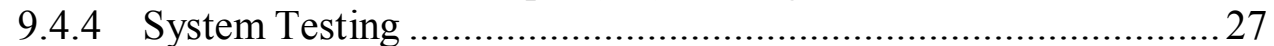

9.5 Inspections and Hold Points ............................................................ 27

9.5.1 Inspection/Test Data ............................................................ 27

9.5.2 Hold Points........................................................................... 28

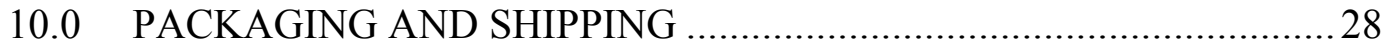

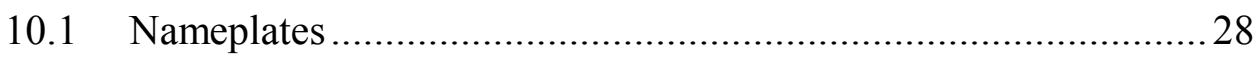

APPENDIX A - VENDOR DATA REQUIREMENTS FORM 414.12A ....

APPENDIX B - DOE-ID AE Standards Appendix M Standard for Piping Materials and Pipe Numbering, Labeling and Color Coding APPENDIX C - Lockheed Martin Idaho Technologies Company Technical Procedures TPR-1187 and TPR1188 


\subsection{SCOPE}

\subsection{Introduction}

This document specifies the minimum requirements for the design, fabrication, testing, supply, system checkout, and delivery of a Heated Vacuum Drying System (HVDS). Any proposed substitutions or exceptions to this specification require the contractor's approval. All of the components of the HVDS can be designed and fabricated from standard off-the-shelf type components and hardware.

The HVDS is used to perform heat and vacuum assisted liquid removal and drying of one to four canisters containing irradiated TMI-2 fuel debris. Each canister may initially contain up to 85 gallons of water but will be dewatered by contractor personnel prior to drying, leaving up to 20 gallons of water to be removed from each of the 342 TMI-2 canisters that will be cycled through the HVDS. However, the HVDS shall be capable of removing 85 gallons of water from each of four canisters concurrently (up to four canisters will be loaded into the HVDS vacuum furnace). The furnace is then sealed and the canisters will then be heated in the vacuum furnace while running the vacuum pump continuously until the required level of dryness is obtained. The fluid removed from the canister(s) is water contaminated with borats, clorides, radionuclides and trace amounts of volatiles. The dried fuel debris canisters will eventually be loaded into a larger dry shielded canister (DSC) for transfer and storage.

This HVDS unit is intended to provide back up assemblies and components for the existing HVDS manufactured by Exolon Systems or it may be installed as a complete parallel system. It shall be functionally identical to the previous unit in all respectsincluding the incorporation of the changes noted in this specification and the referenced sketches and drawings.

\subsection{Work Included}

The work set forth by this specification shall include the following:

- The incorporation of design changes the original HVDS and fabrication of one HVDS complete and ready for use, which meets the performance requirements of this specification. The HVDS shall consist of three assemblies: the vacuum furnace assembly (e.g., vessel, internal spacers, system controller, heating elements, instrumentation, pressure relief, associated hose and fittings); the condenser assembly (e.g., isolation valve, refrigeration unit, associated hose and fittings); the vacuum pump assembly (e.g., motor, filter and instrument panel and ancillary hoses). Design requirements for the three assemblies are given in Section 5. 
- System inspection, operational checkout and acceptance at the subcontractor's shop prior to shipping.

- Documentation including: equipment drawings, as-built shop drawings, data sheets, QA paperwork, PLC (programmable logic controller) and DTAM (operator interface) software and programs in printed and digital format, and operating and maintenance instructions and manuals as specified on Form 414.12A attached.

- Packaging and shipping to the INEEL.

\subsection{Related Work by Others}

The scope of work does not include the following activities or items, which are provided by others:

- Unloading of the HVDS at the INEEL.

- TMI-2 storage or drying system hardware other than the HVDS described in this specification.

- Installation of the HVDS at the INEEL.

\subsection{TECHNICAL TERMINOLOGY}

The following definitions and abbreviations shall apply as used with this specification:

\subsection{Definitions}

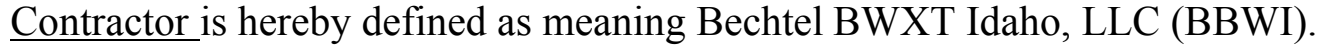

Subcontractor is hereby defined as meaning the entity awarded the contract for furnishing the equipment specified herein.

Work is hereby defined as meaning collectively all work associated with providing the HVDS equipment to be supplied by the subcontractor under the Contract.

Equipment is hereby defined as meaning collectively and all components, materials, items and parts, or portions thereof to be supplied by the subcontractor under the Contract.

Mixed Waste is waste comprised of hazardous and radioactive material as defined in 40CFR 260.10 and Department of Energy Order 0435.1, respectively. 
Commercial Grade Item an item that is:

A. Not subject to design or specification requirements unique to nuclear facilities

B. Used in applications other then nuclear facilities

C. Ordered from manufacture or subcontractor based on specifications published in the manufactures product description (PDD-1; NQA-1-1994, part 1; DOE/RW0333P)

Hold Point is a point which fabrication cannot proceed without contractor approval.

\subsection{Abbreviations}

The abbreviations applicable to this specification are as follows:

$\begin{array}{ll}\text { AISC } & \text { American Institute of Steel Construction } \\ \text { ASME } & \text { American Society of Mechanical Engineers } \\ \text { AWS } & \text { American Welding Society } \\ \text { DSC } & \text { Dry Shielded Canister } \\ \text { HVDS } & \text { Heated Vacuum Drying System } \\ \text { IEC } & \text { International Electrotechnical Commission } \\ \text { NEC } & \text { National Electrical Code } \\ \text { NEMA } & \text { National Electrical Manufacturers Association } \\ \text { NFP(A) } & \text { National Fluid Power Association } \\ \text { NFPA } & \text { National Fire Protection Association } \\ \text { P\&ID } & \text { Piping and Instrumentation Diagram } \\ \text { VDS } & \text { Vacuum Drying System } \\ \text { VFA } & \text { Vacuum Furnace Assembly } \\ \text { TC } & \text { Thermocouple }\end{array}$

\subsection{APPLICABLE CODES, STANDARDS, AND REFERENCES}

All services shall be provided and all equipment and materials shall be furnished and tested in accordance with the codes and standards referenced in this section and/or shown on referenced sketch 1-5. The current issue of the following documents (unless otherwise noted) shall form part of the specification.

\subsection{National Standards}

ANSI AMERICAN NATIONAL STANDARDS INSTITUTE

ANSI Y14.5M Dimensioning and Tollerancing 
Project Title: $\quad$ TMI-2 FUEL VACUUM DRYER SPECIFICATION

Document Type: Procurement Specification Project Number: N/A

SPC: 287

ASME

AMERICAN SOCIETY FOR MECHANICAL ENGINEERS

ASME NQA-1 Quality Assurance Requirements for Nuclear Facility

Applications

ASME B1.20.1 Pipe Threads, General

ASME B31.3 Process Piping

ASME Section V NDE

ASME Section VIII Division 1 Pressure Vessel Code

ASME Section IX Welding and Brazing Qualifications

AISC AMERICAN INSTITUTE OF STEEL CONSTRUCTION

Manual of Steel Construction

ASNT

AMERICAN SOCIETY FOR NONDESTRUCTIVE TESTING

ASNT SNT-TC-1A Personnel Qualification and Certification in

Nondestructive Testing

AWS

AMERICAN WELDING SOCIETY

AWS A2.4 Standard Symbols for Welding, Brazing and

Nondestructive Examination

AWS A3.0

AWS D1.1

Welding Terms and Definitions

AWS QCI

Structural Welding Code Steel

Standard for AWS Certification of Welding Inspectors

NFPA NATIONAL FIRE PROTECTION ASSOCIATION

NFPA 70 NATIONAL FIRE PROTECTION CODE

IEC INTERNATIONAL ELECTROTECHNICAL COMMISSION

NEC NATIONAL ELECTRICAL CODE

DOE-ID Architectural and Engineering Standards

Appendix M Standard for Piping Materials and Pipe Numbering, Labeling, and Color Coding (For labeling and identification only) 
Project Title:

Document Type: SPC: 287
TMI-2 FUEL VACUUM DRYER SPECIFICATION

Procurement Specification Project Number:

N/A

\subsection{TMI-2 Canister Heated Vacuum Drying System HVDS Drawing List}

\begin{tabular}{|c|c|c|c|c|c|c|c|c|}
\hline $\begin{array}{l}\text { Drawing } \\
\text { No. }\end{array}$ & $\begin{array}{l}\text { Drawing } \\
\text { Type }\end{array}$ & Date & Revision & $\begin{array}{l}\text { EXOLON/SPRINGS } \\
\text { FABRICATION } \\
\text { DWG NO. }\end{array}$ & Date & Revision & No of Sheets & Drawing Title \\
\hline $\mathbf{5 0 3 0 8 2}$ & & & $\mathbf{0}$ & & & & 1 & TAN TMI-2 VACUUM FURNACE LIFT FIXTURE \\
\hline 503106 & & & 1 & & & & 3 & TMI-2 VACUUM FURNACE INSTALLATION \\
\hline $\mathbf{5 0 3 1 0 7}$ & & & $\mathbf{0}$ & & & & 1 & $\begin{array}{l}\text { TAN TMI-2 VACUUM FURNACE SPACER } \\
\text { RING(TASK DELETED - DWG NOT ISSUED) }\end{array}$ \\
\hline 503545 & & & 4 & & & & 2 & TMI-2 AIR SWEEP PIENUM ASSEMBLY \\
\hline 503674 & & & 4 & & & & 1 & $\begin{array}{l}\text { TMI-2 HVDS VACUUM FURNACE SUPPORT } \\
\text { BRACES }\end{array}$ \\
\hline 503699 & & & $\mathbf{0}$ & & & & 1 & HVDS P\&ID (NOT RELEASED) \\
\hline 507657 & & & 1 & & & & 5 & TMI-2 FURNACE LID OPENER ASSEMBLY \\
\hline 507658 & & & 2 & & & & 3 & $\begin{array}{l}\text { TMI-2 FURNACE LID OPENER ASSEMBLY } \\
\text { INSTALLATION }\end{array}$ \\
\hline $\mathbf{5 0 7 7 8 2}$ & & & $\mathbf{0}$ & & & & 1 & $\begin{array}{l}\text { TMI-2 VACUUM FURNACE HEATER } \\
\text { PROTECTOR INSTALLATION }\end{array}$ \\
\hline $\mathbf{5 0 7 7 8 3}$ & & & $\mathbf{0}$ & & & & 1 & $\begin{array}{l}\text { TMI-2 VACUUM FURNACE HEATER } \\
\text { PROTECTOR INSTALLATION ASSEMBLY }\end{array}$ \\
\hline 509821 & ED & & $\mathbf{0}$ & & & & 1 & $\begin{array}{l}\text { TMI-2 HEATED VACUUM DRYING SYSTEM } \\
\text { DEMISTER FILTR FLT3 FILTER END CAPS }\end{array}$ \\
\hline 509822 & ED & & $\mathbf{0}$ & & & & 1 & $\begin{array}{l}\text { TMI-2 HVDS DEMISTER FILTER INSTALLATION } \\
\text { AND SPANNER WRENCH }\end{array}$ \\
\hline 511458 & SP & $\begin{array}{l}\text { Not } \\
\text { released }\end{array}$ & $\mathbf{0}$ & & & & 1 & $\begin{array}{l}\text { TMI-2 HEATED VACUUM DRYING SYSTEM } \\
\text { DPIT1A GAUGE INSTALLTION }\end{array}$ \\
\hline 509284 & ED & & $\mathbf{0}$ & & & & 1 & $\begin{array}{l}\text { TMI-2 VACUUM FURNACE CAINSTER HOLE } \\
\text { COVERS }\end{array}$ \\
\hline 507771 & & & $\mathbf{0}$ & & & & 1 & $\begin{array}{l}\text { TMI-2 HVDS FURNACE LID OPENER SYSTEM } \\
\text { BLOCK DIAGRAM }\end{array}$ \\
\hline 507772 & & & $\mathbf{0}$ & & & & 2 & $\begin{array}{l}\text { TMI-2 HVDS FURNACE LID OPENER SYSTEM } \\
\text { PANEL ASSEMBLY }\end{array}$ \\
\hline $\mathbf{5 0 7 7 7 3}$ & & & $\mathbf{0}$ & & & & 1 & $\begin{array}{l}\text { TMI-2 HVDS FURNACE LID OPENER SYSTEM } \\
\text { WIRING DIAGRAM }\end{array}$ \\
\hline
\end{tabular}


Project Title:

Document Type:

SPC: 287
TMI-2 FUEL VACUUM DRYER SPECIFICATION

Procurement Specification Project Number: N/A

\begin{tabular}{|c|c|c|c|c|c|c|c|c|}
\hline $\begin{array}{l}\text { Drawing } \\
\text { No. }\end{array}$ & $\begin{array}{l}\text { Drawing } \\
\text { Type }\end{array}$ & Date & Revision & $\begin{array}{l}\text { EXOLON/SPRINGS } \\
\text { FABRICATION } \\
\text { DWG NO. }\end{array}$ & Date & Revision & No of Sheets & Drawing Title \\
\hline 509455 & & $5 / 4 / 99$ & $\mathbf{0}$ & & & & 2 & $\begin{array}{l}\text { TMI-2 HVDS REMOTE AIR SWEEP POWER } \\
\text { ENCLOSURE ASSEMBLY AND DETAILS (SK-037) }\end{array}$ \\
\hline 509456 & & $5 / 4 / 99$ & $\mathbf{0}$ & & & & 1 & $\begin{array}{l}\text { TMI-2 HVDS REMOTE AIR SWEEP POWER } \\
\text { ENCLOSURE WIRING DIAGRAM (DWS-SK-037) }\end{array}$ \\
\hline 510299 & & $\begin{array}{l}\text { Not } \\
\text { released }\end{array}$ & & & & & 1 & $\begin{array}{l}\text { TMI-2 GAS SAMPLE MONITORING SYSTEM } \\
\text { P\&ID }\end{array}$ \\
\hline $509547 *$ & & $5 / 26 / 99$ & 3.0 & VDS3CPS1' & 1/18/99 & 2.2 & 1 of 4 & PIPING SCHEMATIC \\
\hline $509548 *$ & & $5 / 26 / 99$ & 3.0 & VDS3CPS2' & 1/18/99 & 2.2 & 2 of 4 & PIPING SCHEMATIC \\
\hline 509549* & & $5 / 26 / 99$ & 3.0 & VDS3CPS3' & $6 / 8 / 98$ & 2.1 & 3 of 4 & PIPING SCHEMATIC \\
\hline $509550 *$ & & $5 / 26 / 99$ & 2.0 & VDS33CPS4' & 1/19/99 & 1.6 & 4 of 4 & PIPING SCHEMATIC \\
\hline 509551* & & $5 / 26 / 99$ & 2.0 & VDS300-VPS' & $2 / 13 / 98$ & 1.3 & 1 of 2 & VPS OUTLINE VIEWS \\
\hline $509552 *$ & & $5 / 26 / 99$ & 2.0 & VDS300-VPS' & $1 / 26 / 99$ & 1.4 & 2 of 2 & VPS OUTLINE VIEWS \\
\hline 509539* & & $5 / 26 / 99$ & 2.0 & VDS300-VPS' & $2 / 13 / 98$ & 1.3 & 1 of 5 & VPS OUTLINE VIEWS \\
\hline 509540* & & $5 / 26 / 99$ & 2.0 & VDS300-VPS' & $2 / 13 / 98$ & 1.3 & 2 of 5 & VPS OUTLINE VIEWS \\
\hline 509541* & & $5 / 26 / 99$ & 2.0 & VDS300-VPS' & $2 / 13 / 98$ & 1.3 & 3 of 5 & VPS OUT LINE FIEWS \\
\hline $509542 *$ & & $5 / 26 / 99$ & 2.0 & VDS300-VPS' & $2 / 13 / 98$ & $\mathbf{1 . 3}$ & 4 of 5 & VPS OUT LINE VIEWS \\
\hline 509543* & & $5 / 26 / 99$ & 1.0 & VDS300-VPS' & JULY 98 & $\mathbf{0 . 0}$ & 5 of 5 & VPS OUTLINE VIEWS \\
\hline $\begin{array}{l}\mathbf{5 0 9 9 5 3 7} \\
*\end{array}$ & & $5 / 26 / 99$ & 2.0 & VDS-VPS-CP' & $2 / 13 / 98$ & $\mathbf{1 . 0}$ & 1 of 2 & VACUUM PUMP SKID CONTROL PANEL \\
\hline $\begin{array}{l}\mathbf{5 0 9 9 5 3 8} \\
*\end{array}$ & & $5 / 26 / 99$ & 2.0 & VDS-VPS-CP' & $1 / 26 / 99$ & $\mathbf{1 . 0}$ & 2 of 2 & VACUUM PUMP SKID CONTROL PANEL \\
\hline $509544 *$ & & $5 / 26 / 99$ & 2.0 & VDS-VCS-CP' & $1 / 26 / 99$ & 1.2 & 1 OF 3 & VACUUM CONDENSER SKID CONTROL PANEL \\
\hline $509545 *$ & & $5 / 26 / 99$ & 2.0 & VDS-VCS-CP' & $1 / 26 / 99$ & 1.2 & 2 OF 3 & VACUUM CONDENSER SKID CONTROL PANEL \\
\hline $509546 *$ & & $5 / 26 / 99$ & 2.0 & VDS-VCS-CP' & $6 / 11 / 98$ & 1.1 & 3 OF 3 & VACUUM CONDENSER SKID CONTROL PANEL \\
\hline $509559 *$ & & $5 / 26 / 99$ & $\mathbf{3 . 0}$ & VDS300-ES' & 1/19/99 & 2.3 & $10 F 8$ & ELECTRICAL SCHEMATIC \\
\hline 509560* & & $5 / 26 / 99$ & 3.0 & VDS300-ES' & $6 / 16 / 99$ & 2.1 & 2 OF 8 & ELECTRICAL SCHEMATIC \\
\hline 509561* & & $5 / 26 / 99$ & 3.0 & VDS300-ES' & 1/19/99 & 2.3 & 3 OF 8 & ELECTRICAL SCHEMATIC \\
\hline $509562 *$ & & $5 / 26 / 99$ & 3.0 & VDS300-ES' & 1/19/99 & 2.3 & 4 OF 8 & ELECTRICAL SCHEMATIC \\
\hline 509563* & & $5 / 26 / 99$ & 3.0 & VDS300-ES' & 1/19/99 & 2.3 & 5 OF 8 & ELECTRICAL SCHEMATIC \\
\hline $509564 *$ & & $5 / 26 / 99$ & 3.0 & VDS300-ES' & 1/19/99 & 2.3 & 6 OF 8 & ELECTRICAL SCHEMATIC \\
\hline $509565 *$ & & $5 / 26 / 99$ & 3.0 & VDS300-ES' & 1/19/99 & 2.3 & 7 OF 8 & ELECTRICAL SCHEMATIC \\
\hline $509566 *$ & & $5 / 26 / 99$ & 3 & VDS300-ES' & 1/19/99 & 2.3 & 8 OF 8 & ELECTRICAL SCHEMATIC \\
\hline $510044 *$ & & $3 / 10 / 00$ & 2 & HEPA17-INEL' & $5 / 26 / 99$ & 1 & 1 & HEPA EXHAUST FILTER \\
\hline
\end{tabular}


Project Title:

Document Type: SPC: 287
TMI-2 FUEL VACUUM DRYER SPECIFICATION

Procurement Specification Project Number: N/A

\begin{tabular}{|c|c|c|c|c|c|c|c|c|}
\hline $\begin{array}{l}\text { Drawing } \\
\text { No. }\end{array}$ & $\begin{array}{l}\text { Drawing } \\
\text { Type }\end{array}$ & Date & Revision & $\begin{array}{l}\text { EXOLON/SPRINGS } \\
\text { FABRICATION } \\
\text { DWG NO. }\end{array}$ & Date & Revision & No of Sheets & Drawing Title \\
\hline & & & & HVF8S' & $10 / 29 / 97$ & 0 & 1 & $\begin{array}{l}\text { HIGH VACUUM INLT FILTER MODEL - FTR2- } \\
300\end{array}$ \\
\hline 509553* & & $5 / 26 / 99$ & 8 & $10000051+$ & & 7 & 1 OF 6 & $\begin{array}{l}\text { STAINLESS VACUUM CHAMBER VESSEL } \\
\text { DETAILS }\end{array}$ \\
\hline 509554* & & $5 / 26 / 99$ & 7 & $10000051+$ & & 6 & 2 OF 6 & $\begin{array}{l}\text { STAINLESS VACUUM CHAMBER VESSEL } \\
\text { DETAILS }\end{array}$ \\
\hline 509555* & & $5 / 26 / 99$ & 7 & $10000051+$ & & 6 & 3 OF 6 & $\begin{array}{l}\text { STAINLESS VACUUM CHAMBER VESSEL } \\
\text { DETAILS }\end{array}$ \\
\hline 509556* & & $5 / 26 / 99$ & 7 & $10000051+$ & & 6 & 4 OF 6 & $\begin{array}{l}\text { STAINLESS VACUUM CHAMBER VESSEL } \\
\text { DETAILS }\end{array}$ \\
\hline 509557* & & $5 / 26 / 99$ & 7 & $10000051+$ & & 6 & 5 OF 6 & $\begin{array}{l}\text { STAINLESS VACUUM CHAMBER VESSEL } \\
\text { DETAILS }\end{array}$ \\
\hline 509558* & & $5 / 26 / 99$ & 7 & $10000051+$ & & 6 & 6 OF 6 & $\begin{array}{l}\text { STAINLESS VACUUM CHAMBER VESSEL } \\
\text { DETAILS }\end{array}$ \\
\hline $\begin{array}{l}\text { Sketch } \\
1^{*}\end{array}$ & & $4 / 20 / 00$ & $\overline{\mathbf{0}}$ & & & & 1 OF 5 & Photos of HVDS Mods \\
\hline $\begin{array}{l}\text { Sketch } \\
2 *\end{array}$ & & $4 / 20 / 00$ & $\mathbf{0}$ & & & & 2 OF 5 & Photos of HVDS Mods \\
\hline $\begin{array}{l}\text { Sketch } \\
3 *\end{array}$ & & $4 / 20 / 00$ & $\mathbf{0}$ & & & & 3 OF 5 & Mechanical Drawings of HVDS Mods \\
\hline $\begin{array}{l}\text { Sketch } \\
\text { 4* }\end{array}$ & & $4 / 20 / 00$ & $\mathbf{0}$ & & & & 4 OF 5 & Mechanical Drawings of HVDS Mods \\
\hline $\begin{array}{l}\text { Sketch } \\
5 *\end{array}$ & & $4 / 20 / 00$ & $\mathbf{0}$ & & & & 5 OF 5 & Mechanical Drawings of HVDS Mods \\
\hline
\end{tabular}

* LMITCO Drawing Number

- EXELON Drawing Number

+ Springs Fabrication Drawing Number 


\subsection{SUBMITTALS}

\subsection{Submittals}

The subcontractor shall submit all data described in this Specification including that identified on the Vendor Data Requirements Form 414.12A attached. Submittals shall be made at the times, in the copy quantities, and for the purposes shown on Form 414.12A. Each submittal must identify the submittal item number, specific component and the Purchase Order number.

For data submitted "for information only", the contractor reserves the right to require corrections and resubmittals of vendor data submitted by the subcontractor as necessary to achieve compliance with the requirements of this specification.

Approval by the contractor of documents submitted by the subcontractor or their subtier supplier shall not relieve the subcontractor of the responsibility for correctness of the content of the documents. Responsibility for conformance with all provisions of contract requirements, including compliance with applicable regulations, codes or standards shall rest with the subcontractor.

\subsubsection{Submittals}

The subcontractor shall provide the following documents to the contractor in accordance with the schedules shown on Form 414.12A attached:

- Quality Assurance Program.

- Manufacturing, Inspection and Test Plan, including detailed test procedures (per paragraphs 8.1 of this specification).

- Detailed shop drawings for fabrication of the HVDS. Drawings shall include an electrical schematic and a complete parts list.

- Detailed electrical system control drawings with interconnecting diagrams, block diagrams, single line electrical schematics, cabling, and terminations. The drawings are to identify contacts, overload breakers, and any special electrical features or sensors. Parts lists shall include the individual components used in the system. Labeling and marking shall identify the source and destination connections of individual wires, cables, and controls (see Appendix B, AE Standard for Labeling.)

- Piping and Instrumentation Diagrams (P\&ID) identifying lines, instruments, and equipment. All components shall be labeled physically and identically designated on the P\&ID (see Appendix B). Existing, valves 
Project Title: Document Type:

SPC: 287
TMI-2 FUEL VACUUM DRYER SPECIFICATION

Procurement Specification Project Number: N/A

and other components are to be numbered identically to the corresponding component as the existing system.

- Assembly drawings in sufficient detail such that normal maintenance and repair activities may be conducted on all subcontractor furnished equipment. Specifically, any through-the-wall connections, heater core connections, etc. shall be detailed.

- Actual Certified Material Test Reports for all materials and weld filler materials used in load bearing, pressure boundary, or containment boundary service. For commercial grade items, a certificate of conformance shall be provided.

- Manufacturer's equipment data sheets for all commercial components including, but not limited to, fittings, valves and tubing.

- Welder and inspection personnel qualification records.

- Complete test, inspection and checkout documentation as specified in Sections 9.3, 9.4 and 9.5. The documentation shall include the test procedures used, the testing/checkout results, and any unsatisfactory conditions encountered along with the resolving/correction documentation.

- One complete set of as-built drawings in electronic form, AutoCAD version 14 or newer.

- Detailed printed and digital copies of the PLC (programmable logic controller) with all rung comments, all internal register addresses and I/O addresses fully identified.

- Detailed printed and digital copies of the DTAM (operator interface) program.

- A complete equipment list for all components of the HVDS and a list of all spare parts showing recommended spare parts. This list shall include name of manufacturer, part number for each part and appropriate technical and QA data. Recommended spare parts shall be supplied with the delivered Vendor Data Schedule.

- Tool control procedures.

- Cleaning procedure-describing methods of cleaning, verification of cleanliness, and maintenance of cleanliness through receipt at the INEEL. 
Project Title: $\quad$ TMI-2 FUEL VACUUM DRYER SPECIFICATION

Document Type: Procurement Specification Project Number: N/A

SPC: 287

- Packaging and handling plan.

\subsubsection{Certificate of Compliance}

The subcontractor shall provide a certificate of compliance for the completed HVDS with the following information:

- Identification of the purchased material or equipment by the subcontractor's purchase order number.

- List of specific codes and standards, which the equipment meets.

- Signature by the authorized QA representative responsible for the quality assurance of the purchased equipment.

\subsection{Deviations}

Consideration of any departures from drawings, specifications, or other contractual requirements shall be requested and recorded on a contractor Interface Document, Form 540.16, and shall be submitted to contractor for approval prior to implementation. The subcontractor shall not proceed with incorporation of changes until contractor approval is received.

\subsection{Release To Ship}

The subcontractor shall withhold shipment of items covered by this procurement until a Contractor Quality Release Form, 414.20, is received from the cognizant subcontractor Quality Representative. A copy of the Contractor Quality Release completed by the subcontractor shall be submitted to the Purchaser with the shipment. This release is to confirm completion of source inspection/test and does not indicate final acceptance.

\subsection{DESIGN}

\subsection{General}

The HVDS design shall be the functionally equivalent to the HVDS supplied to VECTRA for Lockheed Martin Idaho Technologies Co., Contract and incorporate changes noted herein and as depicted on BBWI sketches 1 - 5 and referenced drawings. 


\subsection{Design Requirements}

\subsubsection{General System Requirements}

The HVDS shall be capable of satisfying the following design and operational requirements:

- The HVDS shall be functionally equivalent to the existing HVDS.

- The shell of the vacuum furnace shall be made of Type 316L stainless steel; any tubing, pipe, fittings and fasteners shall be 300 series stainless steel. Any variation from materials used in the existing HVDS shall be submitted for contractor approval prior to use of materials.

- All interfaces and connections between assemblies and with facility utilities shall be configured and located identical to the existing system.

- The HVDS system shall be capable of fully automated operation (using programmable logic control [PLC] technology) following manual initiation up to and including canister dryness acceptance testing (provision in the design shall be made for periodic manual acceptance testing).

- Provisions shall be made for remote system operation a minimum of 150 feet from the vacuum and condenser skids.

- The system shall have an automatic data-logging system identical to the existing system.

- The design shall provide for 1 to 4 TMI-2 canisters to be dried concurrently and shall be designed and fabricated to prevent insertion of greater than 4 TMI-2 canisters.

- System design shall preclude unfiltered effluent from entering the atmosphere.

- Liquid effluents from the system shall not exceed $100^{\circ} \mathrm{F}$ and 20 psig.

- The design shall be capable of providing for a minimum mass flow rate of 100-lb water/hour.

- Canister drying temperatures shall be a nominal $900^{\circ} \mathrm{F}$ and shall not exceed $1000^{\circ} \mathrm{F}$. 
Project Title: Document Type:

SPC: 287
TMI-2 FUEL VACUUM DRYER SPECIFICATION

Procurement Specification Project Number: N/A

- The system and its components shall be designed for a minimum of 1200 temperature and pressure cycles based on 120 drying cycles at 100 hours each over a period of two years.

- Special consideration shall be given to preclude the generation of mixed hazardous waste.

- A skid mounted control transformer for 480 VAC 3-phase to 120 VAC singlephase operation shall be provided. The control transformer(s) shall be mounted in the control box and sized by the subcontractor to adequately serve all 120 VAC components in accordance with NEC requirements.

- Motors and motor starters shall be in accordance with NEMA MG-1 or IEC standards and shall have NEMA 4 weatherproof enclosures. All other exposed electrical equipment, which could potentially be a safety hazard, or could be damaged by application of pressurized water, shall be enclosed in NEMA type 4 water-resistant enclosures.

- Each motor starter shall be minimum NEMA size 1.

- The motors and starters shall be rated and configured for 480 volt, 3-phase, 60-hertz operation $100 \%$ duty cycle.

\subsubsection{System Instrumentation}

The following are minimum instrumentation requirements to support the HVDS:

- Means for demonstrating canister dryness shall be such that two unlikely, independent equipment, system, or administrative failures are required to produce a false indication of canister dryness.

- Two pressure gauges (capacitance manometers) shall be mounted on or near the vacuum furnace and interlocked to provide automatic shutdown of the furnace heater when an overpressure condition is sensed. Equivalent equipment and configuration as existing system shall be used.

- Four thermocouples shall be used to monitor the internal temperature of the furnace in the same configuration as existing. Three additional thermocouples shall be installed in furnace locations approximately opposite thermocouples 1, 2, and 3 shown on Drawing SK \#4 and shall be labeled with an A suffix. The added thermocouples shall be for temperature indication only and shall be provided with a separate cable/connector assembly used only for the parallel HVDS system configuration. Duel thermocouples shall be installed in each of the seven locations and shall be wired such that the thermocouples may be 
changed with out removing the HVDS from its shielding cask. Thermocouples shall be installed with all metallic flex conduit to accommodate thermal expansion.

- Provisions shall be made for selected instrumentation, consistent with the existing HVDS unit; to be remotely located a minimum of 150 feet from the drying skid location.

- One compound pressure gauge and one capacitance manometer shall be mounted on the inlet side of the vacuum pump assembly.

- Furnace temperature control shall be included in the PLC with automatic shutdown capability provided.

- Sensors shall be installed at locations indicated on drawings.

- A thermocouple shall be installed in the inlet stream to the condenser assembly to monitor the flow stream temperature.

\subsubsection{Vacuum Furnace Assembly}

The following are minimum requirements for the vacuum furnace:

- The shell of the furnace shall be made of Type 316L stainless steel.

- The furnace shall be insulated. The insulation and jacket materials composition and thicknesses shall be identical to the original furnace.

- The furnace shall be able to heat 1 to 4 TMI-2 canisters concurrently (each canister envelope shall be a 14" nominal outside diameter and 150" in height). The furnace design and fabrication shall preclude insertion of greater than 4 TMI-2 canisters.

- Furnace shall be capable of supporting (4) 2940-lb TMI-2 fuel canisters.

- The furnace shall fit into a shipping cask with internals removed (51.25" I.D.).

- The furnace, except for piping and electrical conduit, shall be 170 " inside height.

- Canisters placed in the furnace shall maintain a 2-1/2" outside to outside minimum spacing in a square lattice. Spacing shall be maintained by spacer disks with a thickness of at least $1 / 2$ " placed within 4 " of the top and bottom of the canisters (canisters are 150" in height). All guide plates are to have 1/8" 
chamfer on both the top and bottom surfaces of the canister openings. The top guide plates shall be drilled as shown on LMITC drawing 507782 for installation of heater protectors by the contractor.

- Heater protectors shown on LMITC drawing 507783 shall be fabricated and installed as shown on LMITC drawing 507782 by the subcontractor.

- Provisions, as shown on referenced sketch 4 , shall be provided for contractor installation of spacer brackets to allow for furnace installation in alternate casks.

- Electric radiant heat shall be provided using durable elements.

- Maximum furnace temperature shall be $1000^{\circ} \mathrm{F}$ with nominal $900^{\circ} \mathrm{F}$ drying temperature.

- Furnace design pressure shall be $+14.7 \mathrm{psig}$ and 0 psia.

- The furnace shall be equipped with a drain line. The drain valve shall be accessible after the vacuum furnace is installed in a shielded vessel.

- The furnace pressure relief shall have a set pressure of 5 psig and be piped to the vacuum pumps HEPA filter inlet. This valve does not have to be ASME rated and stamped.

- The furnace design and fabrication requirements shall be consistent with the rules for ASME Boiler and Pressure Vessel Code, Section VIII, Division 1. The vessel need not be ASME Code stamped.

- The furnace shall be fully compatible with all existing systems.

\subsubsection{Condenser Assembly}

The following are the minimum requirements for the condenser assembly:

- During initial system testing, the condenser shall be verified to remove $90 \%$ by mass of all water vapor leaving the vacuum furnace before it enters the vacuum pump assembly.

- Condenser operation shall not cause an interruption in the heat and vacuum operations of the HVDS.

- The condenser cooler loop shall be a closed loop system. 
- The condenser assembly shall incorporate a form of capacity control such that it shall not cycle more than four starts per hour. The existing system cycles much more frequently, resulting in premature contactor failure. The unit shall be fully compatible with all existing systems.

\subsubsection{Vacuum Pump Assembly}

The following are minimum requirements for the vacuum pump assembly:

- The pump shall have a minimum rating of $300 \mathrm{cfm}$.

- The vacuum pump inlet filter shall be sized for the vacuum pump and have a nominal capacity capable of removing at least $98 \%$ of all particles 4 micron in size or larger.

- Pump sound level shall be maintained below $85 \mathrm{dBA}$ measured 3 feet from the vacuum pump exhaust gas outlet.

- One $1 / 2$ " helium inlet connection and line shall be provided in the same location as the existing unit.

- Vacuum pump assembly instrumentation shall include one compound gauge, one manometer, and a differential pressure-indicating transmitter, Dwyer series 603A, installed per referenced sketches across FLT4 (Vac-Pump HEPA Filter).

- Design shall maintain oil, if required, and intercooler temperatures within manufacturer's specified limits. An oil-less vacuum pump equivalent to the existing oil type pump, requiring no changes to power, control, or operation is a desired option.

- The vacuum skid shall be capable of drawing the HVDS down to an operating pressure of .1 torr under normal operating conditions.

\subsubsection{Hose Assemblies and Fittings}

The following are minimum requirements for hose assemblies:

- $\quad$ Fitting design and selection shall consider ease of installation and disassembly.

- $\quad$ All fittings shall provide for leak tight connections. 
- The hose assembly connecting the vacuum furnace to the condenser shall have a minimum length of 30 feet.

- The subcontractor shall supply a minimum of 25 feet of 1 " drain hose with a male 1"NPT fitting.

- Hoses shall be supported by appropriate strain relief.

- Hoses shall be routed to minimize interference with lid opening apparatus.

\subsubsection{Vacuum Pump and Condenser Skid Assemblies}

The following are minimum requirements for the vacuum pump and vacuum condenser skid:

- All drying equipment, with the exception of the vacuum furnace, shall be skid mounted in same configuration as original system.

- $\quad$ The vacuum pump and vacuum condenser skids shall be limited to a total footprint of $100 \mathrm{ft}^{2}$ combined.

- The vacuum pump and vacuum condenser skid shall be fabricated from carbon steel structural tube, angle, channel, and/or plate and provided with a non-hazardous, pre-approved, durable protective coating.

- $\quad$ The vacuum pump and vacuum condenser skids shall be capable of protecting and supporting all HVDS components other than the vacuum furnace.

- The vacuum pump and vacuum condenser skids shall be designed so that the vacuum pump, filter, and valves are easily accessible and can be lifted using slings or with a fork-lift.

- All moving components such as drive shafts and gears shall be totally enclosed.

\subsection{ENVIRONMENTAL AND SERVICE CONDITIONS}

\subsection{Ambient Conditions}

All HVDS components shall be capable of operating continuously under any combination of the following process flow:

Ambient Conditions $\quad$ Air, steam, and/or helium 
Project Title:

Document Type:

SPC: 287
TMI-2 FUEL VACUUM DRYER SPECIFICATION

Procurement Specification Project Number: N/A
Temperature

Humidity

Pressure $10^{\circ} \mathrm{F}$ to $120^{\circ} \mathrm{F}$

$5 \%$ to $100 \%$

$1 \mathrm{~atm}$

\subsection{Service Conditions}

All HVDS components shall be capable of pumping fluids from the fuel debris canister with the following properties without damage to their operating characteristics:

$\begin{array}{ll}\text { Temperature } & 10^{\circ} \mathrm{F} \text { to } 1000^{\circ} \mathrm{F} \\ \text { Humidity } & 0 \% \text { to } 100 \% \\ \text { Pressure } & 10 \mathrm{e}-6 \text { torr to } 10 \mathrm{psig}\end{array}$

The portion of the HVDS used to pressure test and provide a vacuum for the DSC shall be capable of pumping fluids with the following properties without damage to their operating characteristics:

$\begin{array}{ll}\text { Temperature } & 10^{\circ} \mathrm{F} \text { to } 400^{\circ} \mathrm{F} \\ \text { Humidity } & 0 \% \text { to } 100 \% \\ \text { Pressure } & 10 \mathrm{e}-6 \text { torr to } 22 \mathrm{psig}\end{array}$

The subcontractor shall ensure that all HVDS internals that come into contact with the pumped fluid shall be fabricated from corrosion resistant materials.

\subsection{MATERIALS}

All HVDS components shall be constructed of materials which are suitable for the environment and functional requirements specified herein. Fitting, valve, gauge, and pump seal materials shall be Viton or other suitable material. A vacuum rated, low vapor pressure sealant shall be used on all connections in the vacuum line. Skids shall be equipped with swivel lift eyes and sized for 5:1 safety factor. Subcontractor shall submit vendor data verifying swivel lift eye rating per form 414.12A.

\subsection{MANUFACTURING/ASSEMBLY}

\subsection{General}

Industry standards referenced in Section 3.1 shall be followed during the fabrication and assembly of the HVDS.

The subcontractor shall prepare, maintain and use an Integrated Manufacturing, Inspection and Test Plan incorporating shop travelers which identifies the 
manufacturing, inspection and/or test steps associated with initial material preparation through and including end item delivery. The Integrated Manufacturing, Inspection and Test Plan shall include the schedule for completion of all work with start and finish dates. The plan shall also identify and apply tentative dates to inspection hold points, including hold points specified by the contractor. The Integrated Manufacturing, Inspection and Test Plan shall be submitted to the contractor for inclusion of contractor specified hold points as referenced in Section 9.5.2.

The subcontractor shall maintain a set of redlined as-built configuration drawings, including actual materials used or purchased. The redlined as-built drawings shall be signed by the subcontractor's designated authority as representing the as-built condition and submitted to the contractor. Prior to shipment of the HVDS assembly, the original design drawings shall have the redlined as-built configuration incorporated, be identified as as-built and shall be submitted to the contractor.

\subsection{Materials}

\subsubsection{Base Material}

The subcontractor shall furnish the required materials identified in this Specification or shown on the drawings. Materials shall comply with the specified codes and standards in accordance with the Specifications and drawing callouts.

\subsubsection{Certified Material Test Reports and Certifications}

- The subcontractor shall obtain actual Certified Material Test Reports (CMTRs) for the materials designated for use in load bearing, pressure boundary, or containment boundary service. The CMTRs shall certify that the material has been inspected and tested in accordance with the material specification and meets the chemical and physical requirements of the material specification for the specific grade. The heat number shall be recorded on the CMTRs and on the material. CMTRs shall be submitted to the contractor in accordance with the Vendor Data Requirements Form 414.12A. For commercial grade items, a certificate of conformance shall be provided.

\subsubsection{Welding Material}

Welding filler metals and applicable codes and standards shall be as specified on the drawing and in this Specification. 
Project Title:

Document Type:

SPC: 287
TMI-2 FUEL VACUUM DRYER SPECIFICATION

Procurement Specification Project Number: N/A

\subsubsection{Welding Filler Metal Certified Material Test Reports}

All filler material used in fabrication for load bearing, pressure boundary or containment welds shall comply with the applicable requirements of ASME or AWS and shall have actual CMTRs issued by the original manufacturer or independent laboratory performing material testing for each lot/heat number. The heat number shall be recorded on the CMTR. The CMTR shall certify that the material has been tested in accordance with requirements of the material specification and that the results of the chemical analysis meet the requirements of the material specification for the ASME or AWS material classification specified.

The subcontractor shall submit a completed weld map/history in accordance with Vendor Data Requirements Form 414.12A certifying that the weld filler material used is of the same heat number marked on the CMTRs.

\subsubsection{Welding Material Handling, Storage and Control}

Except as otherwise specified, welding filler metals and fluxes shall be stored, handled and controlled in accordance with manufacturer's recommendations and with contractor approved procedures.

After welding filler metals have been removed from their original package, they shall be protected or stored so that their characteristics and welding properties are not affected. All consumable welding material shall be kept free of oil, grease, and foreign matter. Precautions shall be taken to minimize absorption of moisture. Tractability to heat or lot numbers shall be maintained.

Handling, storage, and control procedures for welding filler metals and backing material shall be submitted to the contractor in accordance with the Vendor Data Requirements Form 414.12A.

\subsubsection{Gases}

Shielding and purge gases shall be in accordance with applicable weld procedure.

\subsubsection{Liquid Penetrant}

Liquid penetrant materials shall meet the requirements of ASME Section 5, Article 6. 
Project Title: $\quad$ TMI-2 FUEL VACUUM DRYER SPECIFICATION

Document Type: Procurement Specification Project Number: N/A

SPC: 287

\subsubsection{Magnetic Particle}

Magnetic particle materials shall meet the requirements of ASME Section 5, Article 7.

\subsection{Traceability}

\subsubsection{Material Marking}

The subcontractor shall mark all items designated for use in load bearing structures or for pressure or containment boundaries with a unique identifier which is traceable to the original Certified Material Test Reports (CMTRs) or other certification documents which document that the specified requirements have been met. The identifier may be the heat, lot or other original material identification.

When material is subdivided, cut, or machined, or when the original mark must be removed, the unique identifier shall be permanently marked on the item in such a way as to maintain tractability. Temporary tags marked with the identifier may be used for items, which are too small for marking, until these items are permanently installed into the assembly.

Each end of straight lengths of bare welding metal shall be flagged, laser imprinted or permanently stamped to show the welding filler metal AWS Classification and heat number. Spooled welding filler metal shall be tagged on the end of the spool to show the welding filler metal AWS material classification and heat number.

\subsubsection{Material Traceability}

Certified Material Test Reports and other material traceability certifications in accordance with Sections 8.2.1.1 and 8.2.2.1, shall be submitted to the contractor per the Vendor Data Requirements Form 414.12A.

\subsection{Fabrication}

The subcontractor shall perform all operations to fabricate the Vacuum Furnace as called for in this Specification and as shown on the referenced drawings.

\subsubsection{Welding Operations}

Welding shall be performed by welders or welding operators qualified to ASME Section IX or AWS D1.1 as applicable. The subcontractor shall have on file documentation, affidavits, and records of testing and test results which 
Project Title: Document Type:

SPC: 287
TMI-2 FUEL VACUUM DRYER SPECIFICATION

Procurement Specification Project Number: N/A

qualify the welder or welding operator for certification. These records shall be submitted to the contractor in accordance with the Vendor Data Requirements Form 414.12A.

The subcontractor shall assign each welder or welding operator with an identifying number, letter, or symbol to identify the welds performed by individual welders or welding operators.

All welding operations shall be performed using welding procedures qualified in accordance with Section 8.4.1.2.

\subsubsection{Weld History}

The subcontractor shall develop a weld map and weld history report, including the results of each inspection, examination and test, for each assembly designated as Quality Level 2. The weld history shall also include:

- Date the weld was made

- Fit-up inspection

- Base material heat number

- Welder or welding operator identification

- Weld filler material used with heat number

- Inspection method

- Acceptance criteria

- Weld procedure

- Weld joint number

- Inspector identification

The weld history shall be submitted to the contractor for approval in accordance with the Vendor Data Requirements Form 414.12A.

\subsubsection{Welding Processes}

All welding shall be performed using welding processes in accordance with ASME Section IX for stainless steel and AWS D1.1 for carbon steel. The weld procedure specifications and procedure qualification records shall be kept on record by the subcontractor, including revisions and effective dates of implementation, and shall be submitted to the contractor in accordance with the Vendor Data Requirements Form 414.12A. 
Project Title:

Document Type:

SPC: 287
TMI-2 FUEL VACUUM DRYER SPECIFICATION

Procurement Specification Project Number: N/A

\subsubsection{Preparation of Base Metal}

Surfaces within 1 inch of any weld location shall be free of any oil, grease, paint, or other material that would prevent proper welding. Plasma arc or laser beam cutting of material is permitted provided the cut surface is machined or ground a minimum of $1 / 16$ inch to bright metal.

\subsubsection{Welding Requirements}

Welds shall provide a surface that is free from cracks, seams, laps, lamination, and porosity in excess of the acceptance criteria established in accordance with Section 8.4.1.6. Defective welds shall be repaired in accordance with Section 8.4.2. Arc strikes shall be removed by grinding in accordance with Section 8.4.2.4.

\subsubsection{Temporary Welds}

Temporary welds shall be subject to the same welding procedure requirements as the final welds. Temporary welds shall be removed unless otherwise permitted by the contractor. Surfaces at removed temporary welds shall be made flush with the original surface and inspected per Section 8.4.2.1.

\subsubsection{Examination}

All welds shall receive in-process examination and be visual and liquid penetrant or magnetic particle (as applicable) examined in accordance with applicable sections of ASME Section VIII, Division 1 for vessels and AWS D1.1 for static structures. Defective welds shall be repaired in accordance with Section 8.4.2.

\subsubsection{Weld Repairs}

\subsubsection{Defect Removal}

Defects shall be completely removed to clean, sound metal by grinding or other methods. Excavated areas shall be examined by liquid penetrant or magnetic particle, as applicable, to assure defect removal.

\subsubsection{Weld Repair Procedures}

Repairs of load bearing structures, or pressure or containment boundary welds to correct weld defects shall be made using the same procedures as for the original welds. Two repair attempts will be 
Project Title:

Document Type:

SPC: 287
TMI-2 FUEL VACUUM DRYER SPECIFICATION

Procurement Specification Project Number: N/A

allowed on any one weld. Subsequent weld repairs shall be made only after receiving written authorization from the contractor and using weld repair procedures approved by the contractor.

Cutting out and rebeveling, then rewelding is considered to be a weld repair.

\subsubsection{Weld Repair Examination}

Repaired areas shall be examined using the same examination methods and procedures by which the defect was originally detected.

\subsubsection{Arc Strikes}

Blemishes caused by arc strikes shall be ground to a smooth contour but no more than 1/32 inch of the base metal shall be removed. Cracks and Arc strikes extending more that 1/32-inch into the base metal shall be considered as a weld defect and repaired in accordance with Sections 8.4.2.

\subsubsection{Rewelding}

Rewelding shall be performed by welders or welding operators qualified in accordance with Section 8.4.1, using welding procedures qualified in accordance with Section 8.4.1.

The surfaces shall be cleaned before rewelding in accordance with Section 8.4.1.3. Rewelds shall meet all of the requirements for welding in this Specification. Defective rewelds can be grounds for rejection of the entire piece or member.

\subsubsection{Weld Repair Reports}

Weld repair reports shall be submitted to the contractor in accordance with Vendor Data Requirements Form 414.12A. The reports shall include all information required in Section 8.4.1.1 plus the examination procedure, the reason for rejection, the number of repairs performed, and documentation that the weld was repaired and accepted.

\subsubsection{Tools and Equipment}

Tools and equipment used by the subcontractor and tools and equipment used in the fabrication of stainless steel alloys shall be free from contamination. The tools shall be maintained free of grease, carbon steel particles, or any foreign matter detrimental 
Project Title: $\quad$ TMI-2 FUEL VACUUM DRYER SPECIFICATION

Document Type: Procurement Specification Project Number: N/A

SPC: 287

to fabrication. Mechanical cleaning tools used for stainless steel shall not cause carbon steel to be embedded into the surface. Grinding wheel material shall be resin bonded. Metal removal tools, wire brushes, and grinding wheels shall not have been previously used on other materials than stainless steels.

The subcontractor shall establish and maintain identification and control procedures for tools and equipment, including wire brushes and grinding wheels used on stainless steel materials. This includes the procedures for isolation, separation, and identification of stainless steel alloy cleaning and grinding tools. These procedures shall be submitted to the contractor in accordance with Vendor Data Requirements Form 414.12A.

The subcontractor shall submit calibration reports that demonstrate traceability to National Institute of Standards and Technology. This requirement shall be applicable to all inspection, test, and measuring equipment used work performed under this procurement.

\subsection{Equipment Labeling}

All components of the HVDS shall be identified on drawings and other submittals and labeled as shown on LMITCO drawings 5099537, 5099538 and 509944 to 509546, 509552, 509543. (EXOLON drawings VDS-VCS-CP, VDS-VPS-CP, VDS300-VCS, VDS300-VPS). Identification of components shall be identical to corresponding components of the existing HVDS. Markings shall be permanent. Metal tags are the preferred method.

\subsection{Cleaning}

\subsubsection{Cleanliness of Piping, Tubing, Vessel, and Components}

Prior to fabrication, all piping or tubing shall be cleaned both internally and externally using ethanol. The piping or tubing shall be maintained visually free of grease, cutting oils, iron oxides and other physical contaminates to NQA-1, Subpart 2.1, Class C cleanliness during fabrication, assembly, and installation.

The vacuum furnace vessel and other system components shall be inspected visually or by wipe test for NQA-1, Subpart 2.1, Class C cleanliness. Dirt, oil residue, metal chips, or other forms of contamination exceeding Class $\mathrm{C}$ limits shall be removed by contractor approved cleaning methods. Any entrapped water shall be removed. All items subject to internal or external corrosion, or contamination shall be suitably protected with all openings sealed with plugs, caps or other suitable means to maintain cleanliness. 
Project Title: $\quad$ TMI-2 FUEL VACUUM DRYER SPECIFICATION

Document Type: Procurement Specification Project Number: N/A

SPC: 287

\subsection{QUALITY ASSURANCE}

\subsection{Program}

The subcontractor shall document, implement and maintain a written quality assurance program (QAP) for the work described in this Specification. The QAP shall include controls for identifying suspect/counterfeit materials to assure that substandard, misrepresented, of fraudulent materials are not used for the fabrication and assembly of items under this contract.

The QAP shall be submitted to the contractor for approval in accordance with Vendor Data Requirements Form 414.12A prior to contract award. The QAP requirements shall be imposed on all subtier suppliers commensurate with the procurement activity.

\subsection{Acceptance}

Final inspection of the HVDS Equipment will be made following successful completion of inspection and system check-outs, and review and approval of all documentation required by this specification.

\subsection{Non-Destructive Examination}

All piping and structural welds shall receive visual (VT) and liquid penetrant (LP) or magnetic particle (MT), as applicable, examination as described in Section 8.4.1.6.

Nondestructive Examination process controls (materials, personnel, procedures and equipment) shall be in accordance with ASME Section V.

Inspections, examinations, and tests for welds and weldments shall be performed by subcontractor approved, qualified personnel in accordance with Section 9.3.2 using procedures in accordance with Section 8.4.1.6. The Inspection/Examination/Test Reports shall be submitted to the contractor in accordance with Vendor Data Requirements Form 414.12A.

The contractor shall have the right to inspect, examine, and test all welds, and to accept, reject, or demand removal of welds, which are in violation of this Specification or of the applicable welding procedure. The subcontractor shall provide access for this activity.

\subsubsection{Nondestructive Examination Procedures}

The subcontractor shall establish detailed examination procedures in accordance with ASME Section V and acceptance criteria for each 
Project Title: Document Type:

SPC: 287
TMI-2 FUEL VACUUM DRYER SPECIFICATION

Procurement Specification Project Number: N/A

nondestructive examination required in accordance with Section 8.4.1.6 of this Specification to ensure conformance of the work to the contractual requirements. The detailed examination procedures shall be submitted to the contractor in accordance with Vendor Data Requirements Form 414.12A.

\subsubsection{Nondestructive Examination Personnel Qualifications}

Nondestructive examination (including visual examination) personnel shall be qualified for the applicable nondestructive testing method in accordance with the requirements of ASNT SNT-TC-1A Levels I, II, or III. Qualification as an AWS certified weld inspector per AWS QC-I is an acceptable alternative for visual examination.

The subcontractor and subtier suppliers shall maintain documentation, affidavits, and records of testing and test results, which qualified the nondestructive examination personnel including records of education, training, and experience, to validate qualification. Records of certification and qualification shall be submitted to the contractor in accordance with Vendor Data Requirements Form 414.12A.

\subsection{Testing}

\subsubsection{Hydrostatic Test}

The vacuum furnace vessel shall be hydrostatically tested at 27 psig in accordance with ASME Section VIII, Division 1 and consistent with the existing vacuum furnace. The subcontractor shall submit test procedure and test reports in accordance with Vendor Data Requirements Form 414.12A. When hydrostatic testing stainless steel components, the testing medium shall not contain more then $50 \mathrm{ppm}$ chlorides.

\subsubsection{Leak Test}

After completion of the vessel and pipe/tubing assembly and prior to installing the heaters, perform the following tests:

- The entire system shall be leak tight to 10-7 atm-cc/sec under a 10-micron vacuum. Any components with indications of leakage shall be repaired or replaced and re-tested.

- Subcontractor shall perform a pressure rise vacuum test at 0.5 Torr or less held constant for one hour. Any components with indications of leakage shall be repaired or replaced and re-tested. 
Project Title: Document Type:

SPC: 287
TMI-2 FUEL VACUUM DRYER SPECIFICATION

Procurement Specification Project Number: N/A

The subcontractor pneumatic test procedures and test reports shall be submitted to the contractor in accordance with Vendor Data Requirements Form 414.12A.

\subsubsection{Heater and Thermocouple (TC) Testing}

After leak testing and after installation of the Heaters and TC's on the Vacuum Furnace Assembly, the heaters and TC's shall be functionally tested and the test documented.

\subsubsection{System Testing}

Prior to shipment, the system shall be assembled and functionally tested to demonstrate operation. The subcontractor shall prepare and submit the system test procedure for contractor approval. As a minimum, the test procedure shall include verification of all specification requirements and the performance tests identified in this specification and in the attached (Appendix C) TMI-2 Canister Drying Testing and Monitoring Technical Procedures, TPR-1187 Assembly/Disassembly and Checkout and TPR-1188 Functional Testing. The tests shall be "bucket" tests in lieu of use of TMI-2 Canisters, shall be performed with the vacuum furnace free-standing without a cask, and shall recover the condensate to demonstrate condenser efficiency. The system test shall include a minimum of three operating cycles.

\subsection{Inspections and Hold Points}

\subsubsection{Inspection/Test Data}

Results of all actual inspections/tests are to be submitted by the subcontractor to the contractor in suitable form. The inspection/test data required may be dimensional inspection data, functional test data, and/or nondestructive test data. In each case, the data must be able to correlate to the material/items being supplied. The data must include the following information, as applicable:

- Part number

- Drawing number/Specification number

- Serial number

- Lot identification number

- Heat/melt number

- Subcontract/purchase order number

- Each characteristic inspected/tested and the inspection/test sequence

- Each characteristic requirement, including drawing or specification Section reference 
Project Title: Document Type:

SPC: 287
TMI-2 FUEL VACUUM DRYER SPECIFICATION

Procurement Specification Project Number: N/A

- Inspection/test results (attributes or variables data)

- Inspection/test equipment used

- Inspection/test set-up employed

- Inspection/test environment

- Inspector's/tester's/interpreter's name/number

- Supervisor's signature

- Test record for the procedure employed

\subsubsection{Hold Points}

The contractor reserves the right to establish and witness on the manufacturing, inspection, test plan, and shop travelers hold-points as deemed appropriate. As a minimum, the following activities shall be witnessed by the contractor's representative and serve as hold points:

- Verification of critical dimensions as defined on drawing 509554.

- Hydrostatic and Leak testing

- Heater testing

- System functional test

The contractor's representative shall be notified at least 10 working days in advance of each activity.

\subsection{PACKAGING AND SHIPPING}

All items shall be inspected for cleanliness to the requirements of section 8.6.

The HVDS assembly shall be packaged to prevent soiling or physical damage during shipping, handling and outdoor storage. Orientation, lifting points, package gross weight and handling and storage precautions shall be clearly marked on the packages. Handling, packaging and shipping procedures and method for off-loading including Professional Engineer (PE) reviewed and approved rigging diagrams, shall be submitted to the contractor in accordance with Vendor Data Requirements Form 414.12A.

\subsection{Nameplates}

Each major HVDS subcomponent (vacuum furnace, condenser, vacuum pump, motor, circulation pump(s), tanks, etc.) shall have the manufacturer's standard identification tag permanently mounted in a visible location. 
Project Title: $\quad$ TMI-2 FUEL VACUUM DRYER SPECIFICATION

Document Type: Procurement Specification Project Number: N/A

SPC: 287 
The Subcontractor shall furnish to the Contractor the specified number of copies of required vendor data for disposition, sufficiently in advance of the date that the material/equipment/service is required to be delivered and/or completed as defined by the Purchase Order. The Vendor Data Requirements Form summarizes the submittal requirements of the Purchase Order and generally specifies the timing for each required submittal. Vendor data for all material and equipment requiring a disposition shall be submitted, reviewed, assigned a disposition code by the Contractor and returned to the Subcontracter. Unless designated as With Shipment, Vendor data shall be submitted under cover of Contractor Form 540.03, Vendor Data Transmittal and Disposition, to:

Bechtel B\&W Idaho (BBWI), LLC.

Procurement Document Control

P. O. Box 1625

Idaho Falls, ID 83415-3521

Vendor Data shall be legible, reproducible, and comply with all applicable Purchase Order requirements. Vendor data submittals shall not be utilized to request deviations from, or changes to, the Purchase Order. Vendor data shall be submitted on a stand-alone basis. Reference to, or review of, previous submittals is prohibited. Vendor data shall clearly identify the submittal item and the submittal number to which it applies.

The Subcontractor and all lower-tier suppliers shall perform no work for which the vendor data has not been reviewed and dispositioned by the Contractor in accordance with the Vendor Data Requirements.

Vendor data causing any change to design details, layouts, calculations, analysis, test methods, procedures, or any other Purchase Order requirements shall be identified to the Contractor utilizing Form 540.16, Interface Document.

Vendor Data disposition codes are:

'A' - (APPROVED), Related work may proceed.

'B' - (APPROVED W/COMMENTS), Related work may proceed ONLY after comments have been incorporated or otherwise reconciled.

'C' - (DISAPPROVED), Related work shall NOT proceed. Resubmit.

'D' - (INFORMATION ONLY SUBMITTAL - RECEIPT ACKNOWLEDGED), No further action is required. 
Requisition No.:

\begin{tabular}{|c|c|c|c|c|c|c|c|c|c|c|c|c|c|c|}
\hline \multirow{7}{*}{$\begin{array}{c}\text { Item } \\
\text { Number }\end{array}$} & \multirow[b]{2}{*}{ Item Description } & \multirow[b]{2}{*}{$\begin{array}{l}\text { Clause/Article } \\
\text { Reference }\end{array}$} & \multirow[b]{2}{*}{$\begin{array}{l}\text { Specification } \\
\text { Reference }\end{array}$} & \multicolumn{4}{|c|}{ Quantity/Schedule } & \multicolumn{6}{|c|}{ Contractor Reviews } & \multirow[b]{2}{*}{$\begin{array}{c}\text { Contractor } \\
\text { Approval } \\
\text { Required/Info } \\
\text { Only }\end{array}$} \\
\hline & & & & $\frac{c}{T^{\prime}}$ & D & $\mathscr{2}$ & 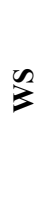 & 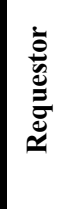 & 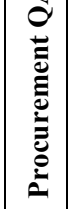 & & 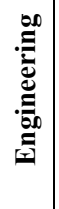 & 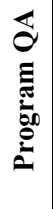 & 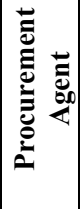 & \\
\hline & Design Verification & & & & & & & & & & & & & \\
\hline & Specification & & & & & & & & & & & & & \\
\hline & Design Calculations & & & & & & & & & & & & & \\
\hline & Characteristic Calculations & & & & & & & & & & & & & \\
\hline & Performance Calculations & & & & & & & & & & & & & \\
\hline \multirow[t]{10}{*}{1} & Test Reports & & $4.1 .1,9.4,9.5$ & & & $\mathrm{X}$ & & $\mathrm{X}$ & $\mathrm{X}$ & $\mathrm{X}$ & $\mathrm{X}$ & $\mathrm{X}$ & $\mathrm{X}$ & A \\
\hline & Correction Calculations & & & & & & & & & & & & & \\
\hline & $\begin{array}{l}\text { Characteristic/Performance Curves - } \\
\square \text { Actual } \square \text { Typical }\end{array}$ & & & & & & & & & & & & & \\
\hline & Characteristic Curves & & & & & & & & & & & & & \\
\hline & Performance Curves & & & & & & & & & & & & & \\
\hline & Test Result Curves & & & & & & & & & & & & & \\
\hline & Calibration Curves & & & & & & & & & & & & & \\
\hline & Operating Curves & & & & & & & & & & & & & \\
\hline & Correction Curves & & & & & & & & & & & & & \\
\hline & Design/Qualification Testing & & & & & & & & & & & & & \\
\hline 2 & Catalog Data & & 4.1.1, 7.0 & & $\mathrm{X}$ & & & $\mathrm{X}$ & $\mathrm{X}$ & $\mathrm{X}$ & $\mathrm{X}$ & $\mathrm{X}$ & $\mathrm{X}$ & A \\
\hline 3 & Engineering Drawings/Reproductions & & 4.1 .1 & $\mathrm{X}$ & & & & $\mathrm{X}$ & $\mathrm{X}$ & $\mathrm{X}$ & $\mathrm{X}$ & $\mathrm{X}$ & $\mathrm{X}$ & A \\
\hline \multirow[t]{3}{*}{4} & Schematics & & 4.1 .1 & $\mathrm{X}$ & & & & $\mathrm{X}$ & $\mathrm{X}$ & $X$ & $\mathrm{X}$ & $\mathrm{X}$ & $X$ & A \\
\hline & Block Diagrams & & & & & & & & & & & & & \\
\hline & Piping Plan & & & & & & & & & & & & & \\
\hline
\end{tabular}

\begin{tabular}{|c|c|c|c|c|c|}
\hline BFR - Before Fabrication Release & $\begin{array}{l}\text { BU - Before Use By } \\
\text { Subcontracter }\end{array}$ & PS - Prior To Shipment & WS - With Shipment & A - Approval Required & I - Information Only \\
\hline
\end{tabular}


Requisition No.:

\begin{tabular}{|c|c|c|c|c|c|c|c|c|c|c|c|c|c|c|}
\hline \multirow[b]{2}{*}{$\begin{array}{l}\text { Item } \\
\text { Number }\end{array}$} & \multirow[b]{2}{*}{ Item Description } & \multirow[b]{2}{*}{$\begin{array}{l}\text { Clause/Article } \\
\text { Reference }\end{array}$} & \multirow[b]{2}{*}{$\begin{array}{l}\text { Specification } \\
\text { Reference }\end{array}$} & \multicolumn{4}{|c|}{ Quantity/Schedule } & \multicolumn{6}{|c|}{ Contractor Reviews } & \multirow[b]{2}{*}{$\begin{array}{c}\text { Contractor } \\
\text { Approval } \\
\text { Required/Info } \\
\text { Only }\end{array}$} \\
\hline & & & & 啚 & ๑ & $\mathscr{E}$ & $\sum^{\infty}$ & 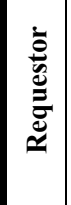 & 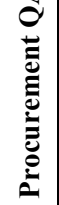 & 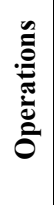 & 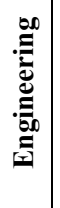 & 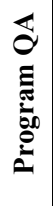 & 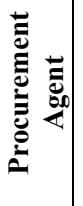 & \\
\hline \multirow[t]{2}{*}{5} & Piping Elevation & & 4.1 .1 & $\mathrm{X}$ & & & & $\mathrm{X}$ & $\mathrm{X}$ & $\mathrm{X}$ & $\mathrm{X}$ & $\mathrm{X}$ & $\mathrm{X}$ & A \\
\hline & Foundation Plan & & & & & & & & & & & & & \\
\hline 6 & Elevation Drawing & & 4.1 .1 & $\mathrm{X}$ & & & & $\mathrm{X}$ & $\mathrm{X}$ & $\mathrm{X}$ & $\mathrm{X}$ & $\mathrm{X}$ & $\mathrm{X}$ & A \\
\hline 7 & General Arrangement Drawings & & 4.1 .1 & $\mathrm{X}$ & & & & $\mathrm{X}$ & $\mathrm{X}$ & $X$ & $\mathrm{X}$ & $\mathrm{X}$ & $\mathrm{X}$ & A \\
\hline 8 & Structural Detail Drawings & & 4.1 .1 & $\mathrm{X}$ & & & & $\mathrm{X}$ & $\mathrm{X}$ & $\mathrm{X}$ & $\mathrm{X}$ & $\mathrm{X}$ & $\mathrm{X}$ & $\mathrm{A}$ \\
\hline 9 & Assembly Drawing & & 4.1 .1 & $\mathrm{X}$ & & & & $\mathrm{X}$ & $\mathrm{X}$ & $\mathrm{X}$ & $\mathrm{X}$ & $\mathrm{X}$ & $\mathrm{X}$ & A \\
\hline 10 & Interface Drawing & & 4.1 .1 & $\mathrm{X}$ & & & & $\mathrm{X}$ & $\mathrm{X}$ & $\mathrm{X}$ & $\mathrm{X}$ & $\mathrm{X}$ & $\mathrm{X}$ & A \\
\hline \multirow[t]{2}{*}{11} & Flow Diagrams (P. \& I.D.) & & 4.1 .1 & $\mathrm{X}$ & & & & $\mathrm{X}$ & $\mathrm{X}$ & $\mathrm{X}$ & $\mathrm{X}$ & $\mathrm{X}$ & $\mathrm{X}$ & A \\
\hline & Panel Cutout Drawings & & & & & & & & & & & & & \\
\hline \multirow[t]{4}{*}{12} & Original As Built Tracings & & 4.1 .1 & & & $\mathrm{X}$ & & $\mathrm{X}$ & $\mathrm{X}$ & $\mathrm{X}$ & $\mathrm{X}$ & $\mathrm{X}$ & $\mathrm{X}$ & I \\
\hline & Weight of Unit & & & & & & & & & & & & & \\
\hline & Descriptive Data & & & & & & & & & & & & & \\
\hline & Connection Drawing & & & & & & & & & & & & & \\
\hline 13 & Weld Map and History & & $4.1 .1,8.4 .1 .1$ & & & $\mathrm{X}$ & & $\mathrm{X}$ & $\mathrm{X}$ & $\mathrm{X}$ & $\mathrm{X}$ & $\mathrm{X}$ & $\mathrm{X}$ & I \\
\hline \multirow[t]{2}{*}{14} & Manufacturing/Inspection/Test Plan & & $4.1 .1,8.1,9.5 .1$ & $\mathrm{X}$ & & & & $\mathrm{X}$ & $\mathrm{X}$ & $X$ & $\mathrm{X}$ & $\mathrm{X}$ & $\mathrm{X}$ & A \\
\hline & Traceability Procedure & & & & & & & & & & & & & \\
\hline 15 & Bill of materials & & 4.1 .1 & $\mathrm{X}$ & & & & $\mathrm{X}$ & $\mathrm{X}$ & $\mathrm{X}$ & $\mathrm{X}$ & $\mathrm{X}$ & $\mathrm{X}$ & A \\
\hline \multirow[t]{3}{*}{16} & Cleaning Procedure & & $4.1 .1,8.6 .1$ & & $\mathrm{x}$ & & & $\mathrm{X}$ & $\mathrm{X}$ & $\mathrm{X}$ & $\mathrm{X}$ & $\mathrm{X}$ & $\mathrm{X}$ & A \\
\hline & Heat Test Procedure & & & & & & & & & & & & & \\
\hline & Sensitive leak Test Procedure & & & & & & & & & & & & & \\
\hline 17 & Hydrostatic Test Procedure & & $4.1 .1,9.4 .1$ & & $\mathrm{X}$ & & & $\mathrm{X}$ & $\mathrm{X}$ & $\mathrm{X}$ & $\mathrm{X}$ & $\mathrm{X}$ & $\mathrm{X}$ & A \\
\hline
\end{tabular}

BFR - Before Fabrication Release $\quad$ BU - Before Use By

PS - Prior To Shipment

WS - With Shipment

A - Approval Required

I - Information Only 
Requisition No.:

\begin{tabular}{|c|c|c|c|c|c|c|c|c|c|c|c|c|c|c|}
\hline \multirow[b]{2}{*}{$\begin{array}{l}\text { Item } \\
\text { Number }\end{array}$} & \multirow[b]{2}{*}{ Item Description } & \multirow[b]{2}{*}{$\begin{array}{l}\text { Clause/Article } \\
\text { Reference }\end{array}$} & \multirow[b]{2}{*}{$\begin{array}{l}\text { Specification } \\
\text { Reference }\end{array}$} & \multicolumn{4}{|c|}{ Quantity/Schedule } & \multicolumn{6}{|c|}{ Contractor Reviews } & \multirow[b]{2}{*}{$\begin{array}{c}\text { Contractor } \\
\text { Approval } \\
\text { Required/Info } \\
\text { Only }\end{array}$} \\
\hline & & & & $\frac{\mathscr{x}}{x}$ & 象 & $\mathscr{E}$ & $\sum$ & 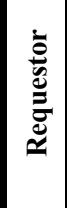 & 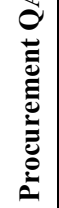 & 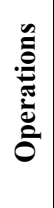 & 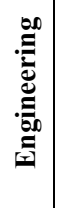 & 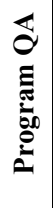 & 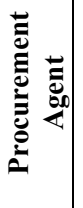 & \\
\hline 18 & Pneumatic Test Procedure & & $4.1 .1,9.4 .2$ & & $\mathrm{X}$ & & & $\mathrm{X}$ & $\mathrm{X}$ & $\mathrm{X}$ & $\mathrm{X}$ & $\mathrm{X}$ & $\mathrm{X}$ & A \\
\hline 19 & Liquid Penetrate Test procedure & & $4.1 .1,9.3 .1$ & & $\mathrm{X}$ & & & $\mathrm{X}$ & $\mathrm{X}$ & & & $\mathrm{X}$ & $\mathrm{X}$ & $\mathrm{A}$ \\
\hline \multirow[t]{3}{*}{20} & Magnetic Particle Test Procedure & & 4.1.1, 9.3.1 & & $\mathrm{X}$ & & & $\mathrm{X}$ & $\mathrm{X}$ & & & $\mathrm{X}$ & $\mathrm{X}$ & A \\
\hline & Radiographic Procedure & & & & & & & & & & & & & \\
\hline & Ultrasonic Test Procedure & & & & & & & & & & & & & \\
\hline 21 & Visual Examination Procedure & & 4.1.1, 9.3.1 & & $\mathrm{X}$ & & & & & & & & & \\
\hline 22 & Weld Procedure/Qualification & & 4.1.1, 8.4.1.2 & & $\mathrm{X}$ & & & $\mathrm{X}$ & $\mathrm{X}$ & & $\mathrm{X}$ & $\mathrm{X}$ & $\mathrm{X}$ & A \\
\hline \multirow[t]{4}{*}{23} & Welder Performance Personnel Certification & & 4.1.1, 8.4.1 & & $\mathrm{X}$ & & & $\mathrm{X}$ & $\mathrm{X}$ & & & $\mathrm{X}$ & $\mathrm{X}$ & A \\
\hline & Hi Pot Dielectric Insulation Test Procedure & & & & & & & & & & & & & \\
\hline & Continuity/Resistance Test Procedure & & & & & & & & & & & & & \\
\hline & Free Iron Test Procedure & & & & & & & & & & & & & \\
\hline \multirow[t]{3}{*}{24} & Calibration Procedure & & $4.11,9.4 .1$ & & $\mathrm{X}$ & & & $\mathrm{X}$ & $\mathrm{X}$ & & $\mathrm{X}$ & $\mathrm{X}$ & & A \\
\hline & Inspection/Test Procedure & & & & & & & & & & & & & \\
\hline & Weld Joint Test Specimens & & & & & & & & & & & & & \\
\hline \multirow[t]{6}{*}{25} & Non Destructive Examination Personnel Certifications & & 4.1.1, 9.3.2 & & $\mathrm{X}$ & & & $\mathrm{X}$ & $\mathrm{X}$ & & & $\mathrm{X}$ & $\mathrm{X}$ & A \\
\hline & Inspector Certifications & & & & & & & & & & & & & \\
\hline & Test Certifications & & & & & & & & & & & & & \\
\hline & Serialization Procedure & & & & & & & & & & & & & \\
\hline & Inspection Test Data & & & & & & & & & & & & & \\
\hline & Limited Shelf Life/Operational Life Data & & & & & & & & & & & & & \\
\hline 26 & Recommended Spares & & 4.1 .1 & & & $\mathrm{X}$ & & $X$ & $X$ & $X$ & $X$ & $X$ & $X$ & I \\
\hline
\end{tabular}

BFR - Before Fabrication Release 
Requisition No.:

\begin{tabular}{|c|c|c|c|c|c|c|c|c|c|c|c|c|c|c|}
\hline \multirow[b]{2}{*}{$\begin{array}{l}\text { Item } \\
\text { Number }\end{array}$} & \multirow[b]{2}{*}{ Item Description } & \multirow[b]{2}{*}{$\begin{array}{l}\text { Clause/Article } \\
\text { Reference }\end{array}$} & \multirow[b]{2}{*}{$\begin{array}{l}\text { Specification } \\
\text { Reference }\end{array}$} & \multicolumn{4}{|c|}{ Quantity/Schedule } & \multicolumn{6}{|c|}{ Contractor Reviews } & \multirow[b]{2}{*}{$\begin{array}{c}\text { Contractor } \\
\text { Approval } \\
\text { Required/Info } \\
\text { Only }\end{array}$} \\
\hline & & & & 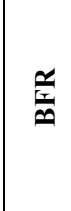 & D & $\mathscr{2}$ & $\sum$ & 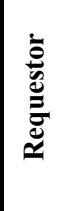 & 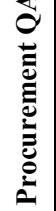 & 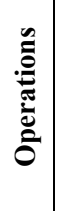 & 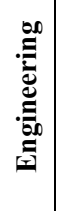 & 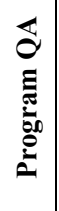 & 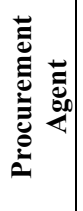 & \\
\hline & Manufacturer's Manuals & & & & & & & & & & & & & \\
\hline & Special Tools List & & & & & & & & & & & & & \\
\hline & Installation Manual (including drawings) & & & & & & & & & & & & & \\
\hline & Maintenance Manual & & & & & & & & & & & & & \\
\hline & Operating Manual & & & & & & & & & & & & & \\
\hline 27 & $\begin{array}{l}\text { Chemical/Physical Test Reports - } \\
\text { xActual } \square \text { Typical }\end{array}$ & & $\begin{array}{l}\text { 4.1.1, 8.2.1.1, } \\
\text { 8.2.2.1 }\end{array}$ & & $\mathrm{X}$ & & & $\mathrm{X}$ & $\mathrm{X}$ & & $\mathrm{X}$ & $\mathrm{X}$ & $\mathrm{X}$ & A \\
\hline 28 & Certificate of Conformance & & $8.2 .1 .1,4.1 .1$ & & $\mathrm{X}$ & & & $\mathrm{X}$ & $\mathrm{X}$ & & $\mathrm{X}$ & $\mathrm{X}$ & $\mathrm{X}$ & A \\
\hline 29 & Special Packaging, Shipping, and Rigging Procedures & & $4.1 .1,10.0$ & & $\mathrm{X}$ & & & $\mathrm{X}$ & $\mathrm{X}$ & $\mathrm{X}$ & $\mathrm{X}$ & $\mathrm{X}$ & $\mathrm{X}$ & A \\
\hline \multirow[t]{3}{*}{30} & Certification of Materials to ASME Code & & $\begin{array}{l}\text { 4.1.1,8.2.4, } \\
8.2 .5\end{array}$ & & $\mathrm{X}$ & & & $\mathrm{X}$ & $\mathrm{X}$ & & & $\mathrm{X}$ & $\mathrm{X}$ & A \\
\hline & Certificate of Disposal or Destruction & & & & & & & & & & & & & \\
\hline & \multicolumn{14}{|l|}{ Additional Submittal Requirements } \\
\hline 31 & Detailed PLC Program (printed \& digital) & & 4.1 .1 & & & $\mathrm{X}$ & & $\mathrm{X}$ & $\mathrm{X}$ & $\mathrm{X}$ & $\mathrm{X}$ & $\mathrm{X}$ & $\mathrm{X}$ & A \\
\hline 32 & $\begin{array}{l}\text { Detailed DTAM (operator interface) Program (printed \& } \\
\text { digital) }\end{array}$ & & 4.1 .1 & & & $\mathrm{X}$ & & $\mathrm{X}$ & $\mathrm{X}$ & $\mathrm{X}$ & $\mathrm{X}$ & $\mathrm{X}$ & $\mathrm{X}$ & A \\
\hline 33 & $\begin{array}{l}\text { Analysis of water for Cl- used in hydrostatic testing of } \\
\text { stainless steel }\end{array}$ & & 4.1.1, 9.4.1 & & $\mathrm{X}$ & & & $\mathrm{X}$ & $\mathrm{X}$ & & $\mathrm{X}$ & $\mathrm{X}$ & & A \\
\hline 34 & System Test Procedure & & $4 \cdot 1 \cdot 1,5 \cdot 2 \cdot 4,9.4 .4$ & & $\mathrm{X}$ & & & $\mathrm{X}$ & $\mathrm{X}$ & $\mathrm{X}$ & $\mathrm{X}$ & $\mathrm{X}$ & $\mathrm{X}$ & A \\
\hline
\end{tabular}


Project Title:

Document Type:

SPC: 287
TMI-2 FUEL VACUUM DRYER SPECIFICATION

Procurement Specification Project Number:

$\mathrm{N} / \mathrm{A}$

\author{
APPENDIX B \\ Standard \\ for \\ Piping Materials \\ and \\ Pipe Numbering, Labeling, and Color Coding
}




\section{STANDARD \\ for \\ PIPING MATERIALS \\ and \\ PIPE NUMBERING, LABELING AND COLOR CODING \\ PREFACE}

This document consists of a Piping Material for each Line Class. The Piping Material Service Code/Line Class/Color Code/Fluid Category Index lists piping system service codes and identifies the Line Class or Classes that are applicable to each service code. New piping systems at INEEL facilities that require a pipe identification system shall use the Index guidelines unless otherwise directed in specific facility or project design criteria. Facilities with other piping identification systems will provide the Design Organization with a labeling scheme to be used on the design documents.

The Piping Material Standards provide a set of standard piping materials and components based upon criteria which has been established for piping systems at ICPP. The standard materials are recommended for new work at other INEEL facilities and are required for new work at SMC. Each service material designated can be referenced to an applicable code. See Sections 1540 through 1543 for the applicable code.

The piping material standards along with the body of the DOE-ID AE Standards and the applicable code provide a basic guideline to specifying piping materials. These standards do not substitute for education, experience and sound engineering judgement. The material standards are to be considered a starting point and in no way relieve the designer from researching original installation specifications on existing facilities and performing all appropriate piping stress analysis on new designs and specifying piping materials accordingly.

Evaluation of a system may require specification of a more appropriate material than those listed in the material standards. At ICPP and SMC lesser material specifications can only be used upon written approval from appropriate facility representatives. For example, at ICPP or SMC, the Design Engineering Organization may approve alternate piping materials.

For new construction at the FAST facility the various FAST piping specifications shall be used for piping design purposes. See ICPP Design Engineering Organization for more information on FAST specifications. 
PIPING MATERIAL

SERVICE CODE / PIPING LINE CLASS / COLOR CODING / FLUID CATEGORY INDEX

\begin{tabular}{|c|c|c|c|c|c|c|}
\hline $\begin{array}{l}\text { SERVICE } \\
\text { CODE }\end{array}$ & SERVICE & $\begin{array}{l}\text { LINE } \\
\text { CLASS }\end{array}$ & MATERIAL & $\begin{array}{c}\text { COLORS } \\
\text { BACKGROUND } \\
\text { LETTER } \\
\end{array}$ & $\begin{array}{l}\text { ASME B31 } \\
\text { FLUID } \\
\text { CATEGORY } \\
\end{array}$ & NOTES \\
\hline AA. & Atmospheric Air & $\mathrm{AR}$ & Stainless Steel 304L & Blue/White & $\mathrm{D}$ & \\
\hline $\mathrm{AC}$ & Acetylene & $\mathrm{AR}$ & Stainless Steel 304L & Yellow/Black & Base & \\
\hline $\mathrm{AH}$ & $\begin{array}{l}\text { Ammonium } \\
\text { Hydroxide }\end{array}$ & $\mathrm{AR}$ & Stainless Steel 304L & Yellow/Black & Base & (1) \\
\hline AM & Air-Methane & $\mathrm{AR}$ & Stainless Steel 304L & Blue/White & $\mathrm{D}$ & (d) \\
\hline AN & Aluminum Nitrate & $\begin{array}{l}\mathrm{AR} \\
\mathrm{HA} \\
\end{array}$ & $\begin{array}{l}\text { Stainless Steel 304L } \\
\text { Hastelloy G-30 R } \\
\end{array}$ & Yellow/Black & Base & (1) \\
\hline $\mathrm{AR}$ & Argon & $\mathrm{AR}$ & Stainless Steel 304L & Blue/White & $\mathrm{D}$ & \\
\hline AS & AMSCO & $\begin{array}{l}\text { AR } \\
\mathrm{NN} \\
\end{array}$ & $\begin{array}{l}\text { Stainless Steel 304L } \\
\text { Carbon Steel }\end{array}$ & Yellow/Black & Base & (D) \\
\hline AW & $\begin{array}{l}\text { Analytical Grade } \\
\text { Water }\end{array}$ & NK & Copper Tubing & Green/White & $\mathrm{D}$ & \\
\hline $\mathrm{BA}$ & Breathing Air & $\begin{array}{l}\mathrm{AM} \\
\mathrm{AR} \\
\mathrm{NN}\end{array}$ & $\begin{array}{l}\text { Stainless Steel Tube } \\
\text { Stainless Steel 304L } \\
\text { Carbon Steel }\end{array}$ & Blue/White & $\mathrm{D}$ & \\
\hline $\mathrm{BB}$ & Boiler Blowdown & $\mathrm{NN}$ & Carbon Steel & Yellow/Black & $\begin{array}{l}\text { ASME } \\
\text { B31.1 } \\
\end{array}$ & \\
\hline $\mathrm{BC}$ & Boiler Chemical & $\mathrm{NN}$ & Carbon Steel & Yellow/Black & Base & \\
\hline $\mathrm{BF}$ & Boiler Feedwater & $\mathrm{NN}$ & Carbon Steel & Yellow/Black & $\begin{array}{l}\text { ASME } \\
\text { B31.1 }\end{array}$ & \\
\hline $\mathrm{BR}$ & Chilled Brine Coolant & $\mathrm{NN}$ & Carbon Steel & Green/White & $\mathrm{D}$ & \\
\hline BW & Basin Water & $\mathrm{AR}$ & Stainless Steel 304L & Yellow/Black & $\mathrm{D}$ & (e) \\
\hline $\mathrm{CB}$ & $\begin{array}{l}\text { Emergency HF Acid } \\
\text { Drain }\end{array}$ & & & Yellow/Black & M & \\
\hline $\mathrm{CC}$ & Cask Coolant & $\mathrm{AR}$ & Stainless Steel 304L & Green/White & $\mathrm{D}$ & (e) \\
\hline $\mathrm{CE}$ & Cation Effluent & NF & $\begin{array}{l}\text { Carbon Steel } \\
\text { Polypropylene Lined }\end{array}$ & Green/White & $\mathrm{D}$ & \\
\hline $\mathrm{CG}$ & Cell Off Gas & $\mathrm{AR}$ & Stainless Steel 304L & Yellow/Black & M & (1)(a) \\
\hline CL & Chlorine & NF & $\begin{array}{l}\text { Carbon Steel } \\
\text { Polypropylene Lined }\end{array}$ & Yellow/Black & M & \\
\hline $\mathrm{CN}$ & $\begin{array}{l}\text { Calcium Nitrate } \\
\text { (Dry and Solution) }\end{array}$ & $\mathrm{AR}$ & Stainless Steel 304L & Yellow/Black & $\mathrm{D}$ & \\
\hline $\mathrm{CO}$ & Chromic Oxide & $\mathrm{AR}$ & Stainless Steel 304L & Yellow/Black & BASE & \\
\hline $\mathrm{CO} 2$ & Carbon Dioxide & $\begin{array}{l}\text { AR } \\
\text { NJ }\end{array}$ & $\begin{array}{l}\text { Stainless Steel 304L } \\
\text { Galv. Carbon Steel } \\
\end{array}$ & Blue/White & $\mathrm{D}$ & $\begin{array}{l}(20) \\
(20)\end{array}$ \\
\hline $\mathrm{CP}$ & Cadmium Sulfate & $\begin{array}{l}\mathrm{AR} \\
\mathrm{CA}\end{array}$ & $\begin{array}{l}\text { Stainless Steel 304L } \\
\text { Carpenter } 20 \mathrm{Cb}^{\mathrm{R}}\end{array}$ & Yellow/Black & BASE & \\
\hline $\mathrm{CR}$ & Chilled Water Return & $\mathrm{AR}$ & Stainless Steel 304L & Green/White & $\mathrm{D}$ & \\
\hline
\end{tabular}


PIPING MATERIAL

SERVICE CODE / PIPING LINE CLASS / COLOR CODING / FLUID CATEGORY INDEX

\begin{tabular}{|c|c|c|c|c|c|c|}
\hline $\begin{array}{l}\text { SERVICE } \\
\text { CODE }\end{array}$ & SERVICE & $\begin{array}{l}\text { LINE } \\
\text { CLASS } \\
\end{array}$ & MATERIAL & $\begin{array}{c}\text { COLORS } \\
\text { BACKGROUND } \\
\text { LETTER } \\
\end{array}$ & $\begin{array}{l}\text { ASME B31 } \\
\text { FLUID } \\
\text { CATEGORY }\end{array}$ & NOTES \\
\hline & & $\begin{array}{l}\text { NK } \\
\text { NN }\end{array}$ & $\begin{array}{l}\text { Copper Tubing } \\
\text { Carbon Steel } \\
\end{array}$ & & & \\
\hline CS & Chilled Water Supply & $\begin{array}{l}\text { AR } \\
\text { NK } \\
\text { NN }\end{array}$ & $\begin{array}{l}\text { Stainless Steel } \\
\text { Copper Tubing } \\
\text { Carbon Steel } \\
\end{array}$ & Green/White & $\mathrm{D}$ & \\
\hline CT & Condensate & $\begin{array}{l}\text { AR } \\
\text { HG } \\
\text { NN } \\
\text { NS } \\
\text { NC } \\
\text { NK }\end{array}$ & $\begin{array}{l}\text { Stainless Steel 304L } \\
\text { Hastelloy G-30 } \\
\text { Carbon Steel } \\
\text { Fiberglass Reinf. } \\
\text { Plastic (FRP) } \\
\text { Carbon Steel - Weld } \\
\text { Fittings } \\
\text { Copper Tubing } \\
\end{array}$ & Yellow/Black & $\mathrm{D}$ & $\begin{array}{l}\text { (1) } \\
\text { (6) (10) }\end{array}$ \\
\hline $\mathrm{CU}$ & Common Utility & AR & Stainless Steel 304L & Yellow/Black & BASE & \\
\hline $\mathrm{CW}$ & $\begin{array}{l}\text { Potable Water } \\
\text { (Hot or Cold) }\end{array}$ & $\begin{array}{l}\text { NJ } \\
\text { NK } \\
\text { NN } \\
\text { NR } \\
\text { ND } \\
\text { NW }\end{array}$ & $\begin{array}{l}\text { Galv. Carbon Steel } \\
\text { Copper Tubing } \\
\text { Carbon Steel } \\
\text { Concrete-Lined } \\
\text { Ductile Iron } \\
\text { CPVC } \\
\text { PVC } \\
\end{array}$ & Green/White & UPC & $\begin{array}{l}\text { (c) } \\
\text { (5) (22) } \\
\text { (4) } \\
(3)\end{array}$ \\
\hline CWR & Cooling Water Return & $\begin{array}{l}\text { AR } \\
\text { NK } \\
\text { NN }\end{array}$ & $\begin{array}{l}\text { Stainless Steel 304L } \\
\text { Copper Tubing } \\
\text { Carbon Steel } \\
\end{array}$ & Green/White & $\mathrm{D}$ & (F) \\
\hline CWS & Cooling Water Supply & $\begin{array}{l}\text { AR } \\
\text { NK } \\
\text { NN }\end{array}$ & $\begin{array}{l}\text { Stainless Steel 304L } \\
\text { Copper Tubing } \\
\text { Carbon Steel }\end{array}$ & Green/White & D & (F) \\
\hline DA & Drying Air & $\mathrm{NN}$ & Carbon Steel & Blue/White & $\mathrm{D}$ & \\
\hline $\mathrm{DC}$ & $\begin{array}{l}\text { Decontaminant } \\
\text { Solution }\end{array}$ & $\begin{array}{l}\mathrm{AB} \\
\mathrm{AR} \\
\mathrm{BD} \\
\mathrm{HC} \\
\mathrm{HG} \\
\end{array}$ & $\begin{array}{l}\text { Nitronic } 50^{\mathrm{R}} \\
\text { Stainless Steel } 304 \mathrm{~L} \\
\text { Stainless Steel } 304 \mathrm{~L} \\
10,000 \text { psi rated } \\
\text { Hastelloy C-4 } \\
\text { or C-22 } \\
\text { Hastelloy G-30 } \\
\end{array}$ & Yellow/Black & $\begin{array}{l}\text { BASE OR } \\
\text { M }\end{array}$ & $\begin{array}{l}(15)(f)(g) \\
(1)\end{array}$ \\
\hline $\mathrm{DE}$ & Diatomaceous Earth & $\begin{array}{l}\mathrm{AR} \\
\mathrm{NJ}\end{array}$ & $\begin{array}{l}\text { Stainless Steel 304L } \\
\text { Galv. Carbon Steel }\end{array}$ & Green/White & $\mathrm{D}$ & \\
\hline DG & Dissolver Off-Gas & $\begin{array}{l}\text { AA } \\
\text { AR }\end{array}$ & $\begin{array}{l}\text { Monel } 400^{\mathrm{R}} \\
\text { Stainless Steel 304L } \\
\end{array}$ & Yellow/Black & M & $\begin{array}{l}\text { (a) } \\
\text { (1) } \\
\end{array}$ \\
\hline DL & Diesel oil & $\mathrm{NN}$ & Carbon Steel & Yellow/Black & BASE & \\
\hline DN & Denitrator Product & $\begin{array}{l}\mathrm{AC} \\
\mathrm{AD}\end{array}$ & $\begin{array}{l}\text { Stainless Steel } 347 \\
\text { Stainless Steel Sch } 40 \\
\text { and Sch } 80304 \mathrm{~L}\end{array}$ & Yellow/Black & M & (b) \\
\hline DO & Dodecane & $\mathrm{AR}$ & Stainless Steel 304L & Yellow/Black & BASE & \\
\hline DP & DOP Aerosol & AR & Stainless Steel 304L & Yellow/Black & $\mathrm{M}$ or $\mathrm{D}$ & (h)(a) \\
\hline DW & Demineralized Water & AR & Stainless Steel 304L & Green/White & $\mathrm{D}$ & (1) \\
\hline
\end{tabular}


PIPING MATERIAL

SERVICE CODE / PIPING LINE CLASS / COLOR CODING / FLUID CATEGORY INDEX

\begin{tabular}{|c|c|c|c|c|c|c|}
\hline $\begin{array}{l}\text { SERVICE } \\
\text { CODE }\end{array}$ & SERVICE & $\begin{array}{l}\text { LINE } \\
\text { CLASS }\end{array}$ & MATERIAL & $\begin{array}{c}\text { COLORS } \\
\text { BACKGROUND } \\
\text { LETTER }\end{array}$ & $\begin{array}{l}\text { ASME B31 } \\
\text { FLUID } \\
\text { CATEGORY }\end{array}$ & NOTES \\
\hline EA & Exhaust Air & $\mathrm{AR}$ & Stainless Steel 304L & Blue/White & $\mathrm{D}$ & $(\mathrm{G})(\mathrm{e})$ \\
\hline ED & $\begin{array}{l}\text { Ethylene Diamine } \\
\text { Tetracetic Acid }\end{array}$ & $\begin{array}{l}\mathrm{AR} \\
\mathrm{AD}\end{array}$ & $\begin{array}{l}\text { Stainless Steel 304L } \\
\text { Stainless Steel Sch } 40 \\
\text { and Sch. } 80 \text { 304L }\end{array}$ & Yellow/Black & BASE & \\
\hline FA & Fluidizing Air & $\begin{array}{l}\text { AR } \\
\text { NN }\end{array}$ & $\begin{array}{l}\text { Stainless Steel 304L } \\
\text { Carbon Steel }\end{array}$ & Blue/White & $\mathrm{D}$ & \\
\hline FB & Fluoboric Acid & $\begin{array}{l}\text { CA } \\
\text { HC } \\
\text { NB }\end{array}$ & $\begin{array}{l}\text { Carpenter } 20 \mathrm{Cb}-3^{\mathrm{R}} \\
\text { Hastelloy C- } 4^{\mathrm{R}} \text { or C- } \\
22^{\mathrm{R}} \\
\text { Carbon Steel ECTFE- } \\
\text { Lined }\end{array}$ & Yellow/Black & M & \\
\hline $\mathrm{FL}$ & Fuel Oil & $\begin{array}{l}\text { AR } \\
\text { NN }\end{array}$ & $\begin{array}{l}\text { Stainless Steel 304L } \\
\text { Carbon Steel }\end{array}$ & Yellow/Black & BASE & \\
\hline FM & $\begin{array}{l}\text { Fire Extinguishing } \\
\text { Foam }\end{array}$ & $\mathrm{NN}$ & Carbon Steel & Red/White & NFPA & \\
\hline FP & Filtered Product & AR & Stainless Steel 304L & Yellow/Black & M & (b) \\
\hline FR & Freon & NM & $\begin{array}{l}\text { Copper Tubing } \\
\text { (Refrigerant) }\end{array}$ & Yellow/Black & $\mathrm{D}$ & \\
\hline FW & Fire Water & $\begin{array}{l}\text { AR } \\
\text { NJ } \\
\text { NN } \\
\text { NR } \\
\text { NA }\end{array}$ & $\begin{array}{l}\text { Stainless Steel 304L } \\
\text { Galvanized Carbon } \\
\text { Steel } \\
\text { Carbon Steel } \\
\text { Ductile Iron } \\
\text { PVC per AWWA } \\
\text { C900 }\end{array}$ & Red/White & NFPA & $\begin{array}{l}(21) \\
(14)(21) \\
(5)(14)(21) \\
(14) \\
(19)\end{array}$ \\
\hline GL & $\begin{array}{l}\text { Glycol Solution } \\
\text { Supply }\end{array}$ & $\mathrm{NN}$ & Carbon Steel & Green/White & $\mathrm{D}$ & \\
\hline GR & $\begin{array}{l}\text { Glycol Solution } \\
\text { Return }\end{array}$ & $\mathrm{NN}$ & Carbon Steel & Green/White & $\mathrm{D}$ & \\
\hline GS & Gasoline & $\begin{array}{l}\mathrm{NJ} \\
\mathrm{NN}\end{array}$ & $\begin{array}{l}\text { Galv. Carbon Steel } \\
\text { Carbon Steel }\end{array}$ & Yellow/Black & BASE & \\
\hline HA & $\begin{array}{l}\text { High Pressure Air } \\
100 \#\end{array}$ & $\begin{array}{l}\mathrm{AC} \\
\mathrm{AM} \\
\mathrm{AR} \\
\mathrm{NN}\end{array}$ & $\begin{array}{l}\text { Stainless Steel } 347 \\
\text { Stainless Steel Tube } \\
\text { Stainless Steel 304L } \\
\text { Carbon Steel }\end{array}$ & Blue/White & $\mathrm{E}$ & (1) \\
\hline HB & $\begin{array}{l}\text { High Pressure Air } \\
150 \# \\
\text { (Dry) }\end{array}$ & $\begin{array}{l}\mathrm{AM} \\
\mathrm{AR} \\
\mathrm{NN}\end{array}$ & $\begin{array}{l}\text { Stainless Steel Tube } \\
\text { Stainless Steel 304L } \\
\text { Carbon Steel }\end{array}$ & Blue/White & $\mathrm{D}$ & \\
\hline $\mathrm{HE}$ & Helium & AR & Stainless Steel 304L & Blue/White & $\mathrm{D}$ & \\
\hline $\mathrm{HF}$ & Hydrofluoric Acid & $\begin{array}{l}\mathrm{AA} \\
\mathrm{HC} \\
\mathrm{NB} \\
\mathrm{NF} \\
\mathrm{NU}\end{array}$ & $\begin{array}{l}\text { Monel } 400^{\mathrm{R}} \\
\text { Hastelloy } \mathrm{C}-4^{\mathrm{R}} \text { or C- } \\
22^{\mathrm{R}} \\
\text { Carbon Steel ECTFE } \\
\text { Lined } \\
\text { Carbon Steel } \\
\text { Polypropylene Lined }\end{array}$ & Yellow/Black & M & \\
\hline
\end{tabular}


PIPING MATERIAL

SERVICE CODE / PIPING LINE CLASS / COLOR CODING / FLUID CATEGORY INDEX

\begin{tabular}{|c|c|c|c|c|c|c|}
\hline $\begin{array}{l}\text { SERVICE } \\
\text { CODE }\end{array}$ & SERVICE & $\begin{array}{l}\text { LINE } \\
\text { CLASS }\end{array}$ & MATERIAL & $\begin{array}{c}\text { COLORS } \\
\text { BACKGROUND } \\
\text { LETTER } \\
\end{array}$ & $\begin{array}{l}\text { ASME B31 } \\
\text { FLUID } \\
\text { CATEGORY } \\
\end{array}$ & NOTES \\
\hline & & NV & $\begin{array}{l}\text { Solid PVDF } \\
\text { Carbon Steel PVDF } \\
\text { Lined } \\
\end{array}$ & & & \\
\hline $\mathrm{HG}$ & Halon Gas & $\mathrm{AR}$ & Stainless Steel 304L & Red/White & NFPA & \\
\hline $\mathrm{HH}$ & Hydrogen & $\begin{array}{l}\mathrm{AR} \\
\mathrm{NN}\end{array}$ & $\begin{array}{l}\text { Stainless Steel } \\
\text { Carbon Steel }\end{array}$ & Yellow/Black & BASE & \\
\hline HI & Instrument Air 100\# & $\begin{array}{l}\mathrm{AM} \\
\mathrm{AR} \\
\mathrm{NN}\end{array}$ & $\begin{array}{l}\text { Stainless Steel Tube } \\
\text { Stainless Steel 304L } \\
\text { Carbon Steel } \\
\end{array}$ & Blue/White & $\mathrm{D}$ & \\
\hline HS & High Pressure Steam & $\begin{array}{l}\mathrm{AM} \\
\mathrm{AR} \\
\mathrm{NC} \\
\mathrm{NN}\end{array}$ & $\begin{array}{l}\text { Stainless Steel Tube } \\
\text { Stainless Steel 304L } \\
\text { Carbon Steel Weld } \\
\text { Fittings } \\
\text { Carbon Steel }\end{array}$ & Yellow/Black & $\mathrm{D}$ & $\begin{array}{l}(9)(6)(10) \\
(1)\end{array}$ \\
\hline HW & High Level Waste & $\mathrm{AR}$ & Stainless Steel 304L & Yellow/Black & M & $\begin{array}{l}\text { (a) }(1) \text { See } \\
\text { ALSO PW }\end{array}$ \\
\hline HWR & Hot Water Return & $\begin{array}{l}\mathrm{AD} \\
\mathrm{AR} \\
\mathrm{NN}\end{array}$ & $\begin{array}{l}\text { Stainless Steel Sch. } 40 \\
\text { and Sch. } 80 \text { 304L } \\
\text { Stainless Steel 304L } \\
\text { Carbon Steel }\end{array}$ & Green/White & $\mathrm{D}$ & \\
\hline HWS & Hot Water Supply & $\begin{array}{l}\mathrm{AD} \\
\mathrm{AR} \\
\mathrm{NN}\end{array}$ & $\begin{array}{l}\text { Stainless Steel Sch. } 40 \\
\text { and Sch. } 80 \text { 304L } \\
\text { Stainless Steel 304L } \\
\text { Carbon Steel }\end{array}$ & Green/White & $\mathrm{D}$ & \\
\hline HX & Hexone & $\begin{array}{l}\text { AR } \\
\mathrm{NN} \\
\end{array}$ & $\begin{array}{l}\text { Stainless Steel 304L } \\
\text { Carbon Steel }\end{array}$ & Yellow/Black & BASE & (1) \\
\hline HY & Hydraulic Fluid & $\begin{array}{l}\mathrm{AR} \\
\mathrm{NN}\end{array}$ & $\begin{array}{l}\text { Stainless Steel 304L } \\
\text { Carbon Steel }\end{array}$ & Green/White & BASE & $\begin{array}{l}(1) \\
(11) \\
\end{array}$ \\
\hline JV & Jet Vent & $\begin{array}{l}\text { AR } \\
\text { NN }\end{array}$ & $\begin{array}{l}\text { Stainless Steel 304L } \\
\text { Carbon Steel }\end{array}$ & Yellow/Black & M & $\begin{array}{l}\text { (a) } \\
\text { (16 }\end{array}$ \\
\hline JW & $\begin{array}{l}\text { Boric Acid or } \\
\text { Borated Water }\end{array}$ & $\mathrm{AR}$ & Stainless Steel 304L & Yellow/Black & $\mathrm{D}$ & \\
\hline KR & Kerosene & $\begin{array}{l}\text { AR } \\
\text { NN }\end{array}$ & $\begin{array}{l}\text { Stainless Steel 304L } \\
\text { Carbon Steel }\end{array}$ & Yellow/Black & BASE & \\
\hline LA2 & Low Pressure Air 20\# & $\begin{array}{l}\mathrm{AM} \\
\mathrm{AR} \\
\mathrm{NN}\end{array}$ & $\begin{array}{l}\text { Stainless Steel Tubing } \\
\text { Stainless Steel 304L } \\
\text { Carbon Steel }\end{array}$ & Blue/White & $\mathrm{D}$ & \\
\hline LA5 & Low Pressure Air 50\# & $\begin{array}{l}\mathrm{AM} \\
\mathrm{AR} \\
\mathrm{NN}\end{array}$ & $\begin{array}{l}\text { Stainless Steel Tubing } \\
\text { Stainless Steel 304L } \\
\text { Carbon Steel }\end{array}$ & Blue/White & $\mathrm{D}$ & \\
\hline LH & Air (Humidified) & $\begin{array}{l}\mathrm{AM} \\
\mathrm{AR}\end{array}$ & $\begin{array}{l}\text { Stainless Steel Tubing } \\
\text { Stainless Steel 304L }\end{array}$ & Blue/White & $\mathrm{D}$ & \\
\hline LI2 & Instrument Air 20\# & $\begin{array}{l}\mathrm{AM} \\
\mathrm{AR} \\
\mathrm{NN} \\
\mathrm{NP}\end{array}$ & $\begin{array}{l}\text { Stainless Steel Tubing } \\
\text { Stainless Steel 304L } \\
\text { Carbon Steel } \\
\text { Copper Tube }\end{array}$ & Blue/White & $\mathrm{D}$ & (8) \\
\hline
\end{tabular}


PIPING MATERIAL

SERVICE CODE / PIPING LINE CLASS / COLOR CODING / FLUID CATEGORY INDEX

\begin{tabular}{|c|c|c|c|c|c|c|}
\hline $\begin{array}{l}\text { SERVICE } \\
\text { CODE }\end{array}$ & SERVICE & $\begin{array}{l}\text { LINE } \\
\text { CLASS }\end{array}$ & MATERIAL & $\begin{array}{c}\text { COLORS } \\
\text { BACKGROUND } \\
\text { LETTER } \\
\end{array}$ & $\begin{array}{c}\text { ASME B31 } \\
\text { FLUID } \\
\text { CATEGORY } \\
\end{array}$ & NOTES \\
\hline & & $\mathrm{NQ}$ & Aluminum Tube & & & $(8)$ \\
\hline LI5 & Instrument Air 50\# & $\begin{array}{l}\text { AM } \\
\text { AR } \\
\text { NN } \\
\text { NP } \\
\text { NQ }\end{array}$ & $\begin{array}{l}\text { Stainless Steel Tubing } \\
\text { Stainless Steel 304L } \\
\text { Carbon Steel } \\
\text { Copper Tube } \\
\text { Aluminum Tube }\end{array}$ & Blue/White & $\mathrm{D}$ & $\begin{array}{l}(8) \\
(8) \\
\end{array}$ \\
\hline LM & Limestone & $\mathrm{NN}$ & Carbon Steel & Green/White & $\mathrm{D}$ & \\
\hline LN & Liquid Nitrogen & $\begin{array}{l}\mathrm{AM} \\
\mathrm{AR} \\
\mathrm{NN}\end{array}$ & $\begin{array}{l}\text { Stainless Steel Tube } \\
\text { Stainless Steel 304L } \\
\text { Carbon Steel }\end{array}$ & Yellow/Black & BASE & \\
\hline LO & Lube Oil & $\begin{array}{l}\text { AR } \\
\text { NN }\end{array}$ & $\begin{array}{l}\text { Stainless Steel 304L } \\
\text { Carbon Steel }\end{array}$ & Green/White & BASE & \\
\hline LS & $\begin{array}{l}\text { Low Pressure Steam } \\
\text { ( } 50 \# \text { or less) }\end{array}$ & $\begin{array}{l}\mathrm{AM} \\
\mathrm{AR} \\
\mathrm{NC} \\
\mathrm{NN}\end{array}$ & $\begin{array}{l}\text { Stainless Steel Tube } \\
\text { Stainless Steel 304L } \\
\text { Carbon Steel Weld } \\
\text { Fittings } \\
\text { Carbon Steel } \\
\end{array}$ & Yellow/Black & $\mathrm{D}$ & $\begin{array}{l}(9) \\
(1) \\
(6)(10) \\
\end{array}$ \\
\hline $\mathrm{ME}$ & Methane & & & Yellow/Black & BASE & \\
\hline MG & Makeup Off-Gas & $\begin{array}{l}\mathrm{AB} \\
\mathrm{AD} \\
\mathrm{AR} \\
\mathrm{CA} \\
\mathrm{HC} \\
\mathrm{NF} \\
\mathrm{NV}\end{array}$ & $\begin{array}{l}\text { Nitronic } 50^{\mathrm{R}} \\
\text { Stainless Steel 304L } \\
\text { Stainless Steel 304L } \\
\text { Carpenter } 20 \mathrm{Cb}-3^{\mathrm{R}} \\
\text { Hastelloy C- } 4^{\mathrm{R}} \text { or C- } \\
22^{\mathrm{R}} \\
\text { Carbon Steel } \\
\text { Polypropylene-Lined } \\
\text { Carbon Steel - PVDF } \\
\text { Lined }\end{array}$ & Yellow/Black & $\mathrm{D}$ & \\
\hline $\mathrm{MN}$ & Mercuric Nitrate & $\mathrm{AR}$ & Stainless Steel 304L & Yellow/Black & BASE & $(1)$ \\
\hline NA & Nitric Acid & $\mathrm{AR}$ & Stainless Steel 304L & Yellow/Black & $\begin{array}{l}\text { BASE OR } \\
\mathrm{M}\end{array}$ & $(1)(f)$ \\
\hline NB & Ammonium Nitrate & $\mathrm{AR}$ & Stainless Steel 304L & Yellow/Black & BASE & \\
\hline $\mathrm{NC}$ & $\begin{array}{l}\text { Noncondensibles } \\
\text { (from Evaporator) }\end{array}$ & $\begin{array}{l}\mathrm{AD} \\
\mathrm{NC}\end{array}$ & $\begin{array}{l}\text { Stainless Steel Sch. } 40 \\
\text { and Sch. } 80 \text { 304L } \\
\text { Carbon Steel, Weld } \\
\text { Fittings }\end{array}$ & Yellow/Black & $\mathrm{D}$ & \\
\hline NG & Inert Gas & $\begin{array}{l}\text { AR } \\
\text { AA }\end{array}$ & $\begin{array}{l}\text { Stainless Steel 304L } \\
\text { Monel } 400^{\mathrm{R}}\end{array}$ & Blue/White & $\mathrm{D}$ & (A) \\
\hline NH & $\begin{array}{l}\text { Nitrogen Gas } \\
\text { (Humidified) }\end{array}$ & $\begin{array}{l}\mathrm{AM} \\
\mathrm{AR} \\
\mathrm{NN}\end{array}$ & $\begin{array}{l}\text { Stainless Steel Tube } \\
\text { Stainless Steel 304L } \\
\text { Carbon Steel }\end{array}$ & Blue/White & $\mathrm{D}$ & \\
\hline NM & Ammonium Nitrate & $\mathrm{AR}$ & Stainless Steel 304L & Yellow/Black & $\mathrm{D}$ & $(\mathrm{C})$ \\
\hline $\mathrm{NO}$ & Nitrous Oxide $\left(\mathrm{N}_{2} 0\right)$ & $\mathrm{AR}$ & Stainless Steel 304L & Yellow/Black & $\mathrm{D}$ & \\
\hline NPH & $\begin{array}{l}\text { Normal Paraffin } \\
\text { Hydrocarbon }\end{array}$ & AR & Stainless Steel 304L & Yellow/Black & BASE & \\
\hline
\end{tabular}


PIPING MATERIAL

SERVICE CODE / PIPING LINE CLASS / COLOR CODING / FLUID CATEGORY INDEX

\begin{tabular}{|c|c|c|c|c|c|c|}
\hline $\begin{array}{l}\text { SERVICE } \\
\text { CODE }\end{array}$ & SERVICE & $\begin{array}{l}\text { LINE } \\
\text { CLASS }\end{array}$ & MATERIAL & $\begin{array}{c}\text { COLORS } \\
\text { BACKGROUND } \\
\text { LETTER } \\
\end{array}$ & $\begin{array}{l}\text { ASME B31 } \\
\text { FLUID } \\
\text { CATEGORY } \\
\end{array}$ & NOTES \\
\hline NR & Nitrogen Gas (Dry) & $\begin{array}{l}\mathrm{AM} \\
\mathrm{AR} \\
\mathrm{NN}\end{array}$ & $\begin{array}{l}\text { Stainless Steel Tube } \\
\text { Stainless Steel 304L } \\
\text { Carbon Steel } \\
\end{array}$ & Blue/White & $\mathrm{D}$ & (1) \\
\hline NW & $\begin{array}{l}\text { Nuclear Poisoned } \\
\text { Water }\end{array}$ & $\mathrm{AR}$ & Stainless Steel 304L & Yellow/Black & $\mathrm{D}$ & \\
\hline $\mathrm{ON}$ & Oxygen & $\mathrm{AR}$ & Stainless Steel 304L & Yellow/Black & $\mathrm{D}$ & \\
\hline OW & Organic Waste & $\mathrm{AD}$ & Stainless Steel 304L & Yellow/Black & $\mathrm{M}$ & (a) \\
\hline PA & Process Ash & $\begin{array}{l}\text { AR } \\
\mathrm{NU} \\
\mathrm{NV}\end{array}$ & $\begin{array}{l}\text { Stainless Steel 304L } \\
\text { Solid PVDF } \\
\text { Carbon Steel, PVDF } \\
\text { Lined }\end{array}$ & Yellow/Black & M & (a) \\
\hline PB & Process Bed Material & $\begin{array}{l}\text { AR } \\
\text { NU } \\
\text { NV }\end{array}$ & $\begin{array}{l}\text { Stainless Steel 304L } \\
\text { Solid PVDF } \\
\text { Carbon Steel PVDF } \\
\text { Lined }\end{array}$ & Yellow/Black & M & (a) \\
\hline $\mathrm{PC}$ & $\begin{array}{l}\text { Process Solution } \\
\text { Complexed w/HF } \\
\text { Exposure }\end{array}$ & $\begin{array}{l}\mathrm{AB} \\
\mathrm{AR} \\
\mathrm{HC}\end{array}$ & $\begin{array}{l}\text { Nitronic } 50^{\mathrm{R}} \\
\text { Stainless Steel } 304 \mathrm{~L} \\
\text { Hastelloy C- } 4^{\mathrm{R}} \text { or C- } \\
22^{\mathrm{R}}\end{array}$ & Yellow/Black & M & (a) \\
\hline PE & $\begin{array}{l}\text { Process Equipment } \\
\text { Waste }\end{array}$ & $\begin{array}{l}\mathrm{AR} \\
\mathrm{HC}\end{array}$ & $\begin{array}{l}\text { Stainless Steel } 304 \mathrm{~L} \\
\text { Hastelloy C- } 4^{\mathrm{R}} \text { or C- } \\
22^{\mathrm{R}}\end{array}$ & Yellow/Black & M & (1)(a)(i) \\
\hline PEF & $\begin{array}{l}\text { Process Equipment } \\
\text { Waste w/HF Exposure }\end{array}$ & $\begin{array}{l}\mathrm{AR} \\
\mathrm{HC}\end{array}$ & $\begin{array}{l}\text { Stainless Steel } 304 \mathrm{~L} \\
\text { Hastelloy C-4 } \\
22^{\mathrm{R}} \text { or C- }\end{array}$ & Yellow/Black & M & (a)(i)(H) \\
\hline $\mathrm{PF}$ & Process - Fluoride & & & Yellow/Black & M & (a) \\
\hline PL & $\begin{array}{l}\text { Process Waste Low } \\
\text { Level w/HF Exposure }\end{array}$ & $\begin{array}{l}\text { AR } \\
\mathrm{HC} \\
\mathrm{HG} \\
\mathrm{NL}\end{array}$ & $\begin{array}{l}\text { Stainless Steel 304L } \\
\text { Hastelloy C-4R or C- } \\
22^{\mathrm{R}} \\
\text { Hastelloy G-30 } \\
\text { Polypropylene }\end{array}$ & Yellow/Black & M & (1)(a) \\
\hline POG & Process Off-Gas & & & Yellow/Black & $\mathrm{M}$ & (a) (l) \\
\hline PP & Propane & $\mathrm{NN}$ & Carbon Steel & Yellow/Black & BASE & \\
\hline PR & $\begin{array}{l}\text { Process Heating/ } \\
\text { Cooling }\end{array}$ & $\mathrm{AR}$ & Stainless Steel 304L & Green/White & $\mathrm{D}$ & \\
\hline PS & $\begin{array}{l}\text { Process Solution } \\
\text { (Uncomplexed) }\end{array}$ & $\begin{array}{l}\text { AA } \\
\text { AB } \\
\text { AR } \\
\text { HC } \\
\text { NU } \\
\text { NV }\end{array}$ & $\begin{array}{l}\text { Monel } 400^{\mathrm{R}} \\
\text { Nitronic } 50^{\mathrm{R}} \\
\text { Stainless Steel 304L } \\
\text { Hastelloy C-4 }{ }^{\mathrm{R}} \text { or C- } \\
22^{\mathrm{R}} \\
\text { Solid PVDF } \\
\text { Carbon Steel PVDF } \\
\text { Lined }\end{array}$ & Yellow/Black & M & $\begin{array}{l}\text { (a) } \\
\text { (1) }\end{array}$ \\
\hline PSF & $\begin{array}{l}\text { Process Waste to } \\
\text { Calcination Process }\end{array}$ & & & Yellow/Black & M & (a) \\
\hline PSG & Process System Off- & & & Yellow/Black & M & $(\mathrm{E})(\mathrm{a})$ \\
\hline
\end{tabular}


PIPING MATERIAL

SERVICE CODE / PIPING LINE CLASS / COLOR CODING / FLUID CATEGORY INDEX

\begin{tabular}{|c|c|c|c|c|c|c|}
\hline $\begin{array}{l}\text { SERVICE } \\
\text { CODE }\end{array}$ & SERVICE & $\begin{array}{l}\text { LINE } \\
\text { CLASS }\end{array}$ & MATERIAL & $\begin{array}{c}\text { COLORS } \\
\text { BACKGROUND } \\
\text { LETTER } \\
\end{array}$ & $\begin{array}{c}\text { ASME B31 } \\
\text { FLUID } \\
\text { CATEGORY } \\
\end{array}$ & NOTES \\
\hline & Gas & & & & & \\
\hline PSL & $\begin{array}{l}\text { Process Liquid } \\
\text { Sample }\end{array}$ & $\begin{array}{l}\text { AD } \\
\text { AR } \\
\mathrm{HC} \\
\mathrm{HG}\end{array}$ & $\begin{array}{l}\text { Stainless Steel Sch. } 40 \\
\text { and Sch. } 80304 \mathrm{~L} \\
\text { Stainless Steel } 304 \mathrm{~L} \\
\text { Hastelloy C-4 }{ }^{\mathrm{R}} \text { or C- } \\
22^{\mathrm{R}} \\
\text { Hastelloy G- } 30^{\mathrm{R}}\end{array}$ & Yellow/Black & M & (a) \\
\hline PSS & $\begin{array}{l}\text { Process Scrub } \\
\text { Solution }\end{array}$ & $\mathrm{AR}$ & Stainless Steel 304L & Yellow/Black & M & (a) \\
\hline PU & Purge & $\mathrm{AR}$ & Stainless Steel 304L & Blue/White & $\mathrm{D}$ & \\
\hline PW & $\begin{array}{l}\text { Process Waste High } \\
\text { Level }\end{array}$ & $\begin{array}{l}\mathrm{AB} \\
\mathrm{AR}\end{array}$ & $\begin{array}{l}\text { Nitronic } 50^{\mathrm{R}} \\
\text { Stainless Steel 304L }\end{array}$ & Yellow/Black & M & (a) \\
\hline PWL & $\begin{array}{l}\text { Process Waste Low } \\
\text { Level }\end{array}$ & $\mathrm{AR}$ & Stainless Steel 304L & Yellow/Black & M & (a) \\
\hline $\mathrm{RC}$ & $\begin{array}{l}\text { Recycle Water } \\
\text { (Process) }\end{array}$ & $\mathrm{AR}$ & Stainless Steel 304L & Yellow/Black & M & (a) \\
\hline $\mathrm{RD}$ & Refrigerant Hot Gas & $\begin{array}{l}\text { AR } \\
\text { NN }\end{array}$ & $\begin{array}{l}\text { Stainless Steel 304L } \\
\text { Carbon Steel }\end{array}$ & Yellow/Black & $\mathrm{D}$ & \\
\hline RE & $\begin{array}{l}\text { Analytical Reagent } \\
\text { Chemical }\end{array}$ & $\mathrm{AR}$ & Stainless Steel 304L & Yellow/Black & M & \\
\hline RG & Rare Gas & $\mathrm{AR}$ & Stainless Steel 304L & Yellow/Black & $\begin{array}{l}\text { BASE OR } \\
\mathrm{M}\end{array}$ & (a)(e) \\
\hline RL & Refrigerant Liquid & $\begin{array}{l}\text { AR } \\
\text { NM } \\
\text { NN }\end{array}$ & $\begin{array}{l}\text { Stainless Steel 304L } \\
\text { Copper Tube } \\
\text { (Refrigerant) } \\
\text { Carbon Steel }\end{array}$ & Yellow/Black & $\mathrm{D}$ & \\
\hline RS & Refrigerant Suction & $\begin{array}{l}\text { AR } \\
\text { NM } \\
\text { NN }\end{array}$ & $\begin{array}{l}\text { Stainless Steel 304L } \\
\text { Copper Tube } \\
\text { (Refrigerant) } \\
\text { Carbon Steel }\end{array}$ & Yellow/Black & $\mathrm{D}$ & \\
\hline RW & Raw Water & $\begin{array}{l}\text { AR } \\
\text { NN } \\
\text { NR }\end{array}$ & $\begin{array}{l}\text { Stainless Steel 304L } \\
\text { Carbon Steel } \\
\text { Ductile Iron }\end{array}$ & Green/White & $\mathrm{D}$ & $\begin{array}{l}(14) \\
(5)(14) \\
(14) \\
\end{array}$ \\
\hline SA & Sulfuric Acid & $\begin{array}{l}\mathrm{CA} \\
\mathrm{NF}\end{array}$ & $\begin{array}{l}\text { Carpenter } 20 \mathrm{Cb}^{\mathrm{R}} \\
\text { Carbon Steel } \\
\text { Polypropylene Lined }\end{array}$ & Yellow/Black & BASE & \\
\hline $\mathrm{SC}$ & Sodium Carbonate & $\mathrm{AR}$ & Stainless Steel 304L & Green/White & $\mathrm{D}$ & \\
\hline SFA & Sulfamic Acid & $\begin{array}{l}\mathrm{AD} \\
\mathrm{AR}\end{array}$ & $\begin{array}{l}\text { Stainless Steel Sch. } 40 \\
\text { and Sch. } 80 \text { 304L } \\
\text { Stainless Steel 304L }\end{array}$ & Yellow/Black & BASE & \\
\hline SG & Sampler Off-Gas & $\begin{array}{l}\text { AR } \\
\mathrm{HC} \\
\mathrm{HG}\end{array}$ & $\begin{array}{l}\text { Stainless Steel } 304 \mathrm{~L} \\
\text { Hastelloy C- } 4^{\mathrm{R}} \text { or C- } \\
22^{\mathrm{R}} \\
\text { Hastelloy G- } 30^{\mathrm{R}}\end{array}$ & Yellow/Black & M & (1)(a) \\
\hline SGC & CAM Vacuum System & $\mathrm{AM}$ & Stainless Steel Tube & Yellow/Black & $\mathrm{D}$ & \\
\hline
\end{tabular}


PIPING MATERIAL

SERVICE CODE / PIPING LINE CLASS / COLOR CODING / FLUID CATEGORY INDEX

\begin{tabular}{|c|c|c|c|c|c|c|}
\hline $\begin{array}{l}\text { SERVICE } \\
\text { CODE }\end{array}$ & SERVICE & $\begin{array}{l}\text { LINE } \\
\text { CLASS }\end{array}$ & MATERIAL & $\begin{array}{c}\text { COLORS } \\
\text { BACKGROUND } \\
\text { LETTER }\end{array}$ & $\begin{array}{l}\text { ASME B31 } \\
\text { FLUID } \\
\text { CATEGORY }\end{array}$ & NOTES \\
\hline & & $\mathrm{AR}$ & Stainless Steel 304L & & & \\
\hline SH & Sodium Hydroxide & $\begin{array}{l}\text { AR } \\
\text { NN }\end{array}$ & $\begin{array}{l}\text { Stainless Steel 304L } \\
\text { Carbon Steel }\end{array}$ & Yellow/Black & BASE & \\
\hline SP & $\begin{array}{l}\text { Span Gas } \\
\text { (for Calibration) }\end{array}$ & AR & Stainless Steel 304L & Yellow/Black & See notes & (j) \\
\hline SS & $\begin{array}{l}\text { Sample Station } \\
\text { Utilities }\end{array}$ & $\begin{array}{l}\mathrm{AM} \\
\mathrm{AR}\end{array}$ & $\begin{array}{l}\text { Stainless Steel Tube } \\
\text { Stainless Steel 304L }\end{array}$ & Yellow/Black & BASE & \\
\hline ST & Starting Air & $\mathrm{NN}$ & Carbon Steel & Blue/White & $\mathrm{D}$ & \\
\hline SV & Sanitary Vent & $\begin{array}{l}\text { NJ } \\
\text { NO } \\
\text { NT } \\
\text { NY }\end{array}$ & $\begin{array}{l}\text { Galv. Steel } \\
\text { PVC - DWV } \\
\text { Cast Iron } \\
\text { ABS }\end{array}$ & Blue/White & UPC & $\begin{array}{l}(7)(\mathrm{c}) \\
(11)(12)(13) \\
(11)(12)(13)\end{array}$ \\
\hline SW & Service Waste & $\begin{array}{l}\text { AM } \\
\text { NF } \\
\text { NH } \\
\text { NN } \\
\text { NS }\end{array}$ & $\begin{array}{l}\text { Stainless Steel Tube } \\
\text { Carbon Steel } \\
\text { Polypropylene Lined } \\
\text { Polyethylene } \\
\text { Carbon Steel } \\
\text { FRP }\end{array}$ & Green/White & $\mathrm{D}$ & (e) \\
\hline TA & Transport Air & $\begin{array}{l}\mathrm{AB} \\
\mathrm{AR}\end{array}$ & $\begin{array}{l}\text { Nitronic } 50^{\mathrm{R}} \\
\text { Stainless Steel 304L }\end{array}$ & Blue/White & $\mathrm{D}$ & $\begin{array}{l}(18)(e) \\
(17)\end{array}$ \\
\hline TP & Tributyl Phosphate & $\mathrm{AR}$ & Stainless Steel 304L & Yellow/Black & BASE & \\
\hline TR & Tartaric Acid & $\mathrm{NJ}$ & Galv. Carbon Steel & Green/White & $\mathrm{D}$ & \\
\hline TW & Treated Water & $\begin{array}{l}\text { AM } \\
\text { NJ } \\
\text { NN }\end{array}$ & $\begin{array}{l}\text { Stainless Steel Tube } \\
\text { Galv. Carbon Steel } \\
\text { Carbon Steel }\end{array}$ & Green/White & $\mathrm{D}$ & (1) \\
\hline UV & Utility Vent & $\begin{array}{l}\text { AR } \\
\text { NN }\end{array}$ & $\begin{array}{l}\text { Stainless Steel 304L } \\
\text { Carbon Steel }\end{array}$ & Blue/White & $\mathrm{D}$ & (e) \\
\hline VA & Vacuum Air & $\begin{array}{l}\text { AR } \\
\text { NN }\end{array}$ & $\begin{array}{l}\text { Stainless Steel 304L } \\
\text { Carbon Steel }\end{array}$ & Blue/White & $\mathrm{D}$ & (G) \\
\hline VG & Vessel Off Gas & $\begin{array}{l}\text { AC } \\
\text { AR } \\
\text { HC } \\
\text { HG } \\
\text { NB }\end{array}$ & $\begin{array}{l}\text { Stainless Steel } 347 \\
\text { Stainless Steel } 304 \mathrm{~L} \\
\text { Hastelloy C-4 } 4^{\mathrm{R}} \text { or C- } \\
22^{\mathrm{R}} \\
\text { Hastelloy G-30 } \\
\text { Carbon Steel ECTFE- } \\
\text { Lined }\end{array}$ & Yellow/Black & M & (a)(i) \\
\hline WQ & Sanitary Waste & $\begin{array}{l}\text { NE } \\
\text { NH } \\
\text { NO } \\
\text { NT } \\
\text { NY } \\
\text { NI }\end{array}$ & $\begin{array}{l}\text { Vitrified Clay } \\
\text { Polyethylene } \\
\text { PVC-DWV } \\
\text { Cast Iron } \\
\text { ABS } \\
\text { Concrete }\end{array}$ & Yellow/Black & UPC & (c) \\
\hline WR & Cooling Water Return & & & Green/White & $\mathrm{D}$ & $(\mathrm{B})(\mathrm{F})$ \\
\hline WS & Wash Solution & $\begin{array}{l}\text { AR } \\
\text { NU } \\
\text { NV }\end{array}$ & $\begin{array}{l}\text { Stainless Steel 304L } \\
\text { Solid PVDF } \\
\text { Carbon Steel PVDF }\end{array}$ & Green/White & $\mathrm{D}$ & (F) \\
\hline
\end{tabular}


PIPING MATERIAL

SERVICE CODE / PIPING LINE CLASS / COLOR CODING / FLUID CATEGORY INDEX

\begin{tabular}{|c|c|c|c|c|c|c|}
\hline $\begin{array}{l}\text { SERVICE } \\
\text { CODE }\end{array}$ & SERVICE & $\begin{array}{l}\text { LINE } \\
\text { CLASS }\end{array}$ & MATERIAL & $\begin{array}{c}\text { COLORS } \\
\text { BACKGROUND } \\
\text { LETTER } \\
\end{array}$ & $\begin{array}{c}\text { ASME B31 } \\
\text { FLUID } \\
\text { CATEGORY }\end{array}$ & NOTES \\
\hline & & & Lined & & & \\
\hline XW & Chemical Waste & $\begin{array}{l}\text { AR } \\
\text { ND } \\
\text { NF } \\
\text { NS }\end{array}$ & $\begin{array}{l}\text { Stainless Steel } \\
\text { CPVC } \\
\text { Carbon Steel } \\
\text { Polypropylene Lined } \\
\text { FRP }\end{array}$ & Yellow/Black & M & (1)(i) \\
\hline
\end{tabular}




\section{PIPING MATERIAL \\ SERVICE CODE / PIPING LINE CLASS / COLOR CODING / FLUID CATEGORY INDEX}

\section{GENERAL NOTES:}

(I) In cases of cross connects, such as utility lines to process lines, where the less severe service piping can be contaminated by backfilling from the more severe service, the line class shall follow that of the more severe service back to and including the first normally closed valve on the less severe service line.

(II) Where a line class has not been specified for a specific service code, contact the Facility Design Engineering Organization for recommendations.

(III) For higher pressures the designer has the option of using heavier schedule pipe.

(IV) For corrosion allowance information on piping contact ICPP Materials Development and Technology Group.

(V) This is not a closed listing. New codes will be devised and this Appendix will be updated when needed.

\section{SERVICE CODE/COLOR CODE NOTES:}

(A) This service code shall not be used in the future. Use AR for Argon or NR for Nitrogen.

(B) This service code has been replaced by CWR.

(C) NB shall be the preferred service code for ammonium nitrate in the future.

(D) AS is the accepted service code for AMSCO. AS was also used as a code for ash in Rover, however, this code shall not be used in the future for ash.

(E) POG shall be the preferred service code for process off-gas in the future.

(F) Color code dependent on chemical additives - contact Industrial Safety for guidance.

(G) Color code dependent on contamination levels - contact Health Physics for guidance.

(H) PE was used at FDP for Process Equipment Waste w/ HF Exposure in the original installation, however, to be consistent with the rest of the plant where PE refers to Process Equipment Waste (without HF exposure) all future PEW lines w/ HF exposure shall be coded PEF.

\section{LINE CLASS NOTES:}

(1) Original Plant - Type 347, Since 1956 - Type 304L

(2) AR shall be used on all new installation in NWCF instead of existing NWCF spec. classes AD and $\mathrm{AF}$, however, the designer is cautioned to research the original NWCF spec. and upgrade the requirements of line class AR if required for the particular service. See also the preface to this appendix and notes under line class AR for direction to the designer for upgrading for the intended service.

(3) Cold water outside buildings only. 


\section{PIPING MATERIAL SERVICE CODE / PIPING LINE CLASS / COLOR CODING / FLUID CATEGORY INDEX}

(4) Hot $\left(180^{\circ} \mathrm{F}\right.$ max. $)$ and cold water inside and outside buildings.

(5) For underground utility piping it is suggested that C.S. pipe and welded fittings be used to ensure electrical continuity. Buried pipe to be coated and protected per Cathodic Protection Section of this Standard. All buried utility lines under concrete slabs shall be all welded construction.

(6) All underground steam and condensate piping shall be C.S. with welded fittings. Buried pipe to be protected per Cathodic Protection Section of this Standard.

(7) Galvanized steel pipe shall not be used for sanitary vents underground and shall be kept at least $6 "$ above the ground.

(8) Where tubing and fittings are used in applications having direct or indirect communication with either radioactive or corrosive chemical, they shall be fabricated of austenitic stainless steel as a minimum or a suitable corrosion resistant material.

(9) Steam tracing only.

(10) Carbon steel pipelines that carry steam or condensate and are buried or located in inaccessible areas shall be of Schedule 80 wall thickness and heavy wall fittings shall be used.

(11) 4" pipe size and less.

(12) Limited to structures where combustible construction is allowed.

(13) Not for use under buildings.

(14) For buried fire water lines and buried raw water lines supplying fire water mains, mechanical joints shall be used except under concrete slabs. Raw water lines which supply fire water mains shall be designed using the same design criteria as for fire water lines. See individual line class specification sheets for more detail. Note that mechanical joints must be bonded to pipe for electrical continuity per this standard.

(15) For FPR high pressure decon jumpers only.

(16) Some portions of the jet vent header systems have used carbon steel piping in the past. All future installations on jet vent header systems shall use only austenitic stainless steel piping.

(17) In-cell solids-free.

(18) Containing solids.

(19) For use in underground pressurized fire water lines only. 


\section{PIPING MATERIAL SERVICE CODE / PIPING LINE CLASS / COLOR CODING / FLUID CATEGORY INDEX}

(20) NJ and AR line classes shall be used in general, however, the designer shall modify these line classes for this service code per the requirements of Kidde-Fenwal CO2 Fire Protection System Manual "Standard Specification for Pipe and Fittings Conveying High Pressure Carbon Dioxide". For line class NJ use the specification for "Steel Pipe and Fittings". For line class AR use the equivalent pipe and fitting pressure ratings as found in the specification for "Steel Pipe and Fittings".

(21) For use in aboveground pressurized fire water lines only.

(22) For all new and replacement metallic piping use Class NJ or NK. Class NN shall not be used for any future work.

\section{FLUID CATEGORIZATION NOTES:}

(a) Dependent on location. In limited access areas such as process cells this service can be considered base category and takes precedence over notes (b), (e), (f) and (h).

(b) Although this service does not fit the definition of category $M$ in ASME B31.3 it shall be considered category $\mathrm{M}$ for ICPP application due to the fissionable material hazard.

(c) UPC is the Uniform Plumbing Code.

(d) Equal to or less than $10 \%$ methane for RAL x-ray spectrometer.

(e) GENERAL CATEGORIZATION NOTE: Services with a normal radiological contamination level above $3.6 \times 10^{-6} \mu \mathrm{c} / \mathrm{ml}$ solution shall be considered category $\mathrm{M}$.

(f) Hot nitric acid (above 100EF) shall be considered category M.

(g) May contain a variety of fluids. When the service contains hot nitric acid see note (f).

(h) Category $\mathrm{M}$ on contaminated side of valve. DOP aerosol supply on uncontaminated side of valve is category D.

(i) Some services may have very low contamination and/or chemical concentration levels and could therefore be considered a lower category than indicated. Contact design engineering organization for these cases.

(j) Dependent on particular service being sampled. See fluid category for service being sampled.

(k) GENERAL CATEGORIZATION NOTE: Services in this listing which are within the confines of power/steam generating facilities shall be ASME B31.1.

(m) Service code POG can be considered category D fluid service if threshold contamination limit described in note (e) above is not exceeded. 


\section{PIPING MATERIAL SERVICE CODE / PIPING LINE CLASS / COLOR CODING / FLUID CATEGORY INDEX}

The following is a list of the line classes used in the index. For a complete description of the piping specifications, see individual piping material specification sheets.

$\underline{\text { Line Class }}$

$\underline{\text { Code }}$

AA

$\mathrm{AB}$

$\mathrm{AC}$

$\mathrm{AD}$

$\mathrm{AE}$

$\mathrm{AF}$

AJ

$\mathrm{AM}$

AR

$\mathrm{BD}$

$\mathrm{CA}$

$\mathrm{HC}$

HG

NA

NB

NC

ND

NE

NF

$\mathrm{NG}$

$\mathrm{NH}$

NI

NJ

NK

NL

NM

$\mathrm{NN}$

NO

NP

NQ

NR

NS

NT

NU

NV

NW

NX

NY

NZ $\underline{\text { Material }}$

Monel $400^{\mathrm{R}}$

Nitronic $50^{\mathrm{R}}(11)$

347 SST

304L SST Piping Sch 40 and Sch 80 (1)

Inactive (3)

Inactive (2)

Inactive (4)

304 SST Tubing

304L SST Pipe Sch 40

304L SST Pipe - 10,000 PSI Rated (8)

Carpenter $20 \mathrm{Cb}-3 \mathrm{SST}^{\mathrm{R}}$ (11)

Hastelloy C-4 ${ }^{\mathrm{R}}$ or C-22 ${ }^{\mathrm{R}}$ (10) (11)

Hastelloy G-30 ${ }^{\mathrm{R}}$ (10) (11)

PVC per AWWA C900

Carbon Steel ECTFE-Lined (Halar ${ }^{\mathrm{R}}$ )

Carbon Steel w/SW or BW Fittings

CPVC (6)

Vitrified Clay (7) (11)

Carbon Steel Polypropylene - Lined

Inactive (5)

Polyethylene (11)

Concrete (11)

Galvanized Carbon Steel

Copper Tubing - Type L or K

Polypropylene (11)

Copper Tubing (Refrigerant Service)

Carbon Steel w/SCRD, SW, BW or Flanged Fittings

PVC-DWV (11)

Copper Tubing - Type K

Aluminum Tubing

Ductile Iron, Concrete - Lined (11)

Fiberglass Reinforced Plastic (FRP) (11)

Cast Iron (11)

Solid PVDF $\left(\right.$ Kynar $\left.^{\mathrm{R}}\right)$ (11)

Carbon Steel, PVDF - Lined $\left(\mathrm{Kynar}^{\mathrm{R}}\right)$

PVC

Transite (Prohibited in all new installations) (11)

ABS Plastic Drain, Waste, and Vent (11)

Inactive (9)

\section{NOTES:}

1. $\quad \mathrm{AD}$ was also a line class used on original construction at NWCF. It included both Sch 40 and Sch 80 304L SST pipe. See NWCF Spec. SP-453504-50-3 for reference. It was incorporated 


\section{PIPING MATERIAL SERVICE CODE / PIPING LINE CLASS / COLOR CODING / FLUID CATEGORY INDEX}

into line classes $\mathrm{AD}$ and $\mathrm{AR}$. In general where Sch 80 pipe was used in a line class use line class AD. Use line class AR for Sch 40. At FPR class AD is used for in-cell piping and class AR for out-of-cell piping. Class AD provides for Sch 80 pipe in the 1/2" to 2" pipe sizes to attain corrosion allowances for the 40 year design life of the facility.

2. AF was a line class used on original construction at NWCF. It included both Sch 40 and Sch 80 304L SST pipe. See NWCF Spec. SP-453504-50-3 for reference. It was incorporated into line classes $\mathrm{AD}$ and $\mathrm{AR}$. In general where Sch 80 pipe is used in a line use line class AD. Use line class AR for Sch 40.

3. AE was a line class used on original construction at NWCF. It included Sch 10, 40 and Sch 80 304L SST pipe for oxygen service. See NWCF Spec. SP-453504-50-3 for reference. It was incorporated into line classes $\mathrm{AD}$ and $\mathrm{AR}$.

4. $\quad \mathrm{AJ}$ was an Inconel $^{\mathrm{R}}$ line class. Inconel pipe is no longer used at ICPP.

5. NG was an aluminum line class. Aluminum pipe is no longer used at ICPP.

6. Line class ND, CPVC, shall be used for hot water services in systems where line class NW, $\mathrm{PVC}$, is used for potable water.

7. NE is the designated line class for vitrified clay. NE was used at NWCF for cast iron, however, NT is the designated line class for cast iron. All future work at NWCF shall use NT for cast iron.

8. $\quad$ BD is a line class used at FPR only for jumpers at the cell wall for high pressure decon. This line class is not anticipated to be used elsewhere in the future. No piping specification is provided here as this line class is inactive for future work. See FPR Spec. Package CP-5B Div. 15 Subdivision 15.18 for more details.

9. $\quad \mathrm{NZ}$ was a line class designation for concrete pipe. NI is the preferred line class designation for concrete pipe.

10. HG has been used as a line class designation for Hastelloy C-4 ${ }^{\mathrm{R}} / \mathrm{C}-22^{\mathrm{R}}$ in CPP-601 E-Cell and at FDP. All new installations shall use $\mathrm{HC}$ line class designation for Hastelloy $\mathrm{C}-4^{\mathrm{R}} / \mathrm{C}-22^{\mathrm{R}}$.

11. These materials as well as Nitronic $60^{\mathrm{R}}$ used for bolting materials are not listed in ASME/ANSI B 31.3 for the service intended, however, they are approved for service based on prior experience and additional testing per paragraph 304.7.2. In addition, some of these may be Water Supply and Sewage piping and are listed in the Uniform Plumbing Code. 


\section{PIPING MATERIAL \\ SERVICE CODE / PIPING LINE CLASS / COLOR CODING / FLUID CATEGORY INDEX}

The following are trademarks used throughout the material specification sheets:

Blue Brute - J.M. Manufacturing Co., Inc.

Carpenter $20 \mathrm{Cb} 3$ - Carpenter Technology Corp.

DOW Plastic - Lined Valves, Fittings and Pipe - DOW Chemical Company

Driscopipe - Philips Driscopipe, Inc.

Fluoroflex-K - Resistoflex Corp.

Grafoil - Union Carbide

Gylon - Garlock, Inc.

Halar - Allied Chemical Co., Inc.

Hastelloy C-4 - Haynes International, Inc.

Hastelloy C-22 - Haynes International, Inc.

Hastelloy G-30 - Haynes International, Inc.

Hypalon - E.I. DuPont De Nemours and Co.

Inconel - Huntington Alloys Inc.

Kynar - Pennwalt Corp.

Monel 400 - Huntington Alloys, Inc.

Nitronic 50 - Armco, Inc.

Nitronic 60 - Armco, Inc.

Nupro - Crawford Fitting Company

R-Con Pipe Connector - R-Con International, Inc.

Superproline - Asahi/America Inc.

Swagelok - Crawford Fitting Company

Tefzel - E.I. DuPont De Nemours and Co.

Triple-Lok - Parker-Hannifin Corp.

Viton - E.I. DuPont De Nemours and Co.

Whitey - Crawford Fitting Company 
PIPING MATERIAL STANDARDS

\section{Monel $400^{R}, 150$ psi Service Rating, Line Class AA}

\section{Design Notes}

1. All external components for valves shall be 304, 316, or approved equal Stainless Steel.

2. Where corrosion or erosion will be excessive or higher pressures or other pipe stresses are encountered, the designer has the option of selecting heavier walled pipe and higher rated valves and fittings.

3. When using spiral wound gaskets, see Rules for Bolted Flange Connections, ASME Section VIII Div.1 Appendix 2 or ASME Section VIII Div.2 Appendix 3 and ASME Section III Div. 1, Subsection ND, Section ND-3658 (for equivalent pressure approach) to ensure that the gaskets are applicable for the particular service intended. In cases where spiral wound gaskets are incompatible with the above requirements contact ICPP Design Engineering Organization for further guidance.

\begin{tabular}{|c|c|c|c|}
\hline TYPE & PIPE SIZE & MATERIAL DESCRIPTION & CODE \\
\hline \multirow[b]{2}{*}{ Pipe } & 2" \& Smaller & Seamless Monel $400^{\mathrm{R}}$, Sch $80 \mathrm{~S}$, Annealed. & ASTM B165 UNS NO4400 \\
\hline & $2-1 / 2^{\prime \prime}-8^{\prime \prime}$ & Seamless or welded Monel $400^{\mathrm{R}}$, Sch 40S, Annealed. & ASTM B165 UNS NO4400 \\
\hline \multirow[b]{2}{*}{ Fittings } & 2" \& Smaller & $\begin{array}{l}\text { Seamless Monel } 400^{\mathrm{R}} \text {, Sch } 80 \text { S Butt-Weld Ends, } \\
\text { Annealed. }\end{array}$ & $\begin{array}{l}\text { ASTM B366 WPNCS UNS NO4400 } \\
\text { Dimensions per ANSI B16.9 }\end{array}$ \\
\hline & $2-1 / 2^{\prime \prime}-8^{\prime \prime}$ & $\begin{array}{l}\text { Seamless Monel } 400^{\mathrm{R}} \text {, Sch } 40 \text { S Butt-Weld Ends, } \\
\text { Annealed. }\end{array}$ & $\begin{array}{l}\text { ASTM B3166 WPNCS UNS } \\
\text { NO4400 Dimensions per ANSI } \\
\text { B16.9 }\end{array}$ \\
\hline \multicolumn{2}{|c|}{ Branches } & \multicolumn{2}{|l|}{ Use full or reducing tees or saddles. } \\
\hline \multicolumn{2}{|c|}{ Bolting } & $\begin{array}{l}\text { Use } 304 \text { SST bolts ASTM A } 193 \text { Gr B } 8 \text { and galling } \\
\text { resistant Nitronic } 60^{\mathrm{R}} \text { nuts ASTM A194 Gr } 8 \text { S (UNS } \\
\text { S21800). }\end{array}$ & $\begin{array}{l}\text { ASTM A193 Gr. B8 ASTM A194 } \\
\text { Gr. 8S UNS S21800 }\end{array}$ \\
\hline \multirow[b]{2}{*}{ Flanges } & 2" \& Smaller & $\begin{array}{l}\text { 150\# ANSI B16.5, R.F., Weld Neck, Sch 80S, Monel } \\
400^{\mathrm{R}} \text {, ASTM B127 UNS NO4400, Finished per ASTM } \\
\text { A182. }\end{array}$ & $\begin{array}{l}\text { ASTM B127 UNS NO4400 } \\
\text { Dimensions per ANSI B16.5 Finish } \\
\text { per ASTM A182 }\end{array}$ \\
\hline & $2-1 / 2^{\prime \prime}-8^{\prime \prime}$ & $\begin{array}{l}\text { 150\# ANSI B16.5, R.F., Weld Neck, Sch 40S, Monel } \\
400^{\mathrm{R}} \text {, ASTM B127 UNS NO4400, Finished per ASTM } \\
\text { A182. }\end{array}$ & $\begin{array}{l}\text { ASTM B127 UNS NO4400 } \\
\text { Dimensions per ANSI B16.5 Finish } \\
\text { per ASTM A182 }\end{array}$ \\
\hline \multirow[b]{2}{*}{ Gaskets } & All Sizes & $\begin{array}{l}\text { Spiral Wound Metallic Type, Monel } 400^{\mathrm{R}} \text { Spiral } \\
\text { Windings, Monel } 400^{\mathrm{R}} \text { Centering Guide. }\end{array}$ & API 601 Note 3 \\
\hline & All Sizes & \multicolumn{2}{|l|}{ Select for service } \\
\hline
\end{tabular}




\section{PIPING MATERIAL STANDARDS}

\section{Stainless Steel Nitronic 50 ${ }^{R}, 150$ psi Service Rating, Line Class AB}

\section{Design Notes}

1. All external components for valves shall be 304, 316, or approved equal Stainless Steel.

2. Where corrosion or erosion will be excessive or higher pressures or other pipe stresses are encountered, the designer has the option of selecting heavier walled pipe and higher rated valves and fittings.

3. When using spiral wound gaskets, see Rules for Bolted Flange Connections, ASME Section VIII Div.1 Appendix 2 or ASME Section VIII Div. 2 Appendix 3 and ASME Section III Div.1, Subsection ND, Section ND-3658 (for equivalent pressure approach) to ensure that the gaskets are applicable for the particular service intended. In cases where spiral wound gaskets are incompatible with the above requirements contact ICPP Design Engineering Organization for further guidance.

4. Full port valves are preferred in fouling service.

5. Nitronic $50^{\mathrm{R}}$ preferred, $316 \mathrm{~L}$ only when Nitronic $50^{\mathrm{R}}$ not available.

6. Alternate disc materials which are suitable for the intended service may be substituted to minimize galling between the seat and disc with prior approval of ICPP Design Engineering Organization.

\begin{tabular}{|c|c|c|c|}
\hline TYPE & PIPE SIZE & MATERIAL DESCRIPTION & CODE \\
\hline \multirow[b]{3}{*}{ Pipe } & 2" \& Smaller & $\begin{array}{l}\text { Seamless Nitronic } 50^{\mathrm{R}} \text { Stainless Steel, Sch. 80, Beveled } \\
\text { Ends }\end{array}$ & ASTM-A312 Grade TP XM-19 \\
\hline & $\begin{array}{l}2-1 / 2 " \text { and } \\
\text { Larger }\end{array}$ & Seamless or welded Nitronic $50^{\mathrm{R}}$ Stainless Steel, Sch. 40 . & Same as above \\
\hline & 5" \& Larger & Nitronic $50^{\mathrm{R}}$ Stainless Steel, Sch 40 , Centrifugally Cast & ASTM-A-351 Grade CG6MMN \\
\hline \multicolumn{2}{|c|}{ Fittings } & $\begin{array}{l}\text { Seamless Wrought Stainless Steel, Butt Welding Ends. } \\
\text { Note: } 90 \mathrm{E} \text { and } 45 \mathrm{E} \text { Ells to be used only where space } \\
\text { prohibits the use of bends. Wall thickness shall match } \\
\text { pipe. }\end{array}$ & $\begin{array}{l}\text { ASTM A } 403 \text { Class WP-S Grade } \\
\text { XM-19 ANSI B16.9 }\end{array}$ \\
\hline \multirow[b]{2}{*}{$\begin{array}{c}\text { Gate } \\
\text { Valves } \\
\end{array}$} & $\begin{array}{l}2^{\prime \prime} \text { and } \\
\text { Smaller }\end{array}$ & $\begin{array}{l}\text { 150\# Class BW or SW Ends, OS\&Y, Bolted Bonnet, } \\
\text { Integral Seat, Body, Bonnet and Disc Nitronic } 50^{\mathrm{R}} \text {, } \\
\text { Casting ASTM A351, Grade CG6MMN or } 316 \mathrm{SST} \text {, } \\
\text { Casting ASTM A351, Grade CF8M, Forgings ASTM } \\
\text { A182, Grade F316L. Select packing and gaskets for } \\
\text { service. }\end{array}$ & $\begin{array}{l}\text { ASTM A351 Grade CG6MMN (5) } \\
\text { ASTM A351 Grade CF8M ASTM } \\
\text { A182 Grade F316 ANSI B16.34 } \\
\text { Notes } 1 \text { and } 6\end{array}$ \\
\hline & $\begin{array}{l}1 / 2^{\prime \prime} \text { and } \\
\text { Larger }\end{array}$ & $\begin{array}{l}\text { 150\# Class, 1/2" and RF, OS\&Y, Bolted Bonnet, Integral } \\
\text { Seat, Body Bonnet, and Disc Nitronic 50R } \text {, Casting ASTM } \\
\text { A351, Grade CG6MMN or } 316 \text { SST, Casting ASTM } \\
\text { A351, Grade CF8M, Forgings ASTM A182, Grade F316. } \\
\text { Select packing and gaskets for service. }\end{array}$ & $\begin{array}{l}\text { ASTM A351 Grade CG6MMN (5) } \\
\text { ASTM A351 Grade CF8M ASTM } \\
\text { A182 Grade F316 ANSI B16.34 } \\
\text { Notes } 1 \& 6\end{array}$ \\
\hline $\begin{array}{l}\text { Globe } \\
\text { Valves }\end{array}$ & $1 / 2 "$ to $2^{\prime \prime}$ & $\begin{array}{l}\text { 150\# Class, BW Ends, RF, OS\&Y, Bolted Bonnet, Integral } \\
\text { Seat Body, Bonnet, and Disc Nitronic } 50^{\mathrm{R}} \text {, Casting ASTM } \\
\text { A351, Grade CG6MMN or 316L Stainless Steel, Castings } \\
\text { ASTM A351, Grade CF3M, Forgings ASTM A182, Grade } \\
\text { F316L. Select packing and gaskets for service. }\end{array}$ & $\begin{array}{l}\text { ASTM A351, Grade CG6MMN (5) } \\
\text { ASTM A351 Grade CF3M ASTM } \\
\text { A182 Grade F316L ANSI B16.34 } \\
\text { Notes } 1 \& 6\end{array}$ \\
\hline
\end{tabular}


PIPING MATERIAL STANDARDS

Stainless Steel Nitronic $50^{R}, 150$ psi Service Rating, Line Class AB

\begin{tabular}{|c|c|c|c|}
\hline TYPE & PIPE SIZE & MATERIAL DESCRIPTION & CODE \\
\hline & $\begin{array}{l}1 / 2^{\prime \prime} \text { and } \\
\text { Larger }\end{array}$ & $\begin{array}{l}\text { 150\# Class, RF OS\&Y, Bolted Bonnet, Integral Seat } \\
\text { Body, Bonnet, and Disc Nitronic 50R } \text {, Casting ASTM } \\
\text { A351, Grade CG6MMN or } 316 \text { SST Castings ASTM } \\
\text { A351 Grade CF8M Forgings ASTM A182, Grade F316. } \\
\text { Select packing and gaskets for service. }\end{array}$ & $\begin{array}{l}\text { ASTM A35.1, Grade CG6MMN (5) } \\
\text { ASTM A351 Grade CF8M ASTM } \\
\text { A182 Grade F316 ANSI B16.34 } \\
\text { Notes } 1 \& 6\end{array}$ \\
\hline & $\begin{array}{l}1 / 2^{\prime \prime} \text { and } \\
\text { Larger }\end{array}$ & $\begin{array}{l}\text { 150\# Class, BW ends, SST Bellows-Seal Type, OS\&Y, } \\
\text { Bolted Bonnet, Integral Seat, Body, Bonnet and Disc } \\
\text { Nitronic } 50^{\mathrm{R}} \text {, Casting ASTM A351, Grade CG6MMN or } \\
\text { 316L Stainless Steel. Castings ASTM A351, Grade } \\
\text { CF3M, Forgings ASTM A182, Grade F316L, 3" Long } \\
\text { Minimum Pipe Stub Each End. }\end{array}$ & $\begin{array}{l}\text { ASTM A351 Grade CG6MMN (5) } \\
\text { ASTM A351 Grade CF3M ASTM } \\
\text { A182 Grade F316L ANSI B16.34 } \\
\text { Notes } 1 \& 6\end{array}$ \\
\hline & $1 / 2^{\prime \prime}-2^{\prime \prime}$ & $\begin{array}{l}\text { 160\# Class, BW Ends, Swing Type, Bolted Bonnet, } \\
\text { Integral Seat, Body Bonnet and Disc Nitronic } 50^{\mathrm{R}} \text {, Casting } \\
\text { ASTM A351, Grade CF3M. Forgings ASTM A182, } \\
\text { Grade F316L. }\end{array}$ & $\begin{array}{l}\text { ASTM A351 Grade CG6MMN (5) } \\
\text { ASTM A351 Grade CF3M ASTM } \\
\text { A182 Grade F316L ANSI B16.34 } \\
\text { Notes } 1 \& 6\end{array}$ \\
\hline $\begin{array}{l}\text { Check } \\
\text { Valves }\end{array}$ & $\begin{array}{l}1 / 2^{\prime \prime} \text { and } \\
\text { Larger }\end{array}$ & $\begin{array}{l}\text { 150\# Class, RF, Swing Type, Bolted Bonnet, Integral Seat, } \\
\text { Body, Bonnet and Disc Nitronic 50 }{ }^{\mathrm{R}} \text {, Casting ASTM } \\
\text { A351, Grade CG6MMN or } 316 \text { SST, Castings ASTM } \\
\text { A351, Grade CF8M, Forgings ASTM A182, Grade F316. }\end{array}$ & $\begin{array}{l}\text { ASTM A351 Grade CG6MMN (5) } \\
\text { ASTM A351 Grade CF3M ASTM } \\
\text { A182 Grade F316L ANSI B16.34 } \\
\text { Notes } 1 \& 6 \\
\end{array}$ \\
\hline & $1 / 2^{\prime \prime}-2^{\prime \prime}$ & $\begin{array}{l}\text { 150\# Class, BW Ends, 3-Piece, Solid Body or Split-Body, } \\
\text { Top Entry, Body Nitronic } 50^{\mathrm{R}} \text {, Casting ASTM A351, } \\
\text { Grade CG6MMN or } 316 \mathrm{~L} \text { Stainless Steel, Castings ASTM } \\
\text { A351, Grade CF3M, Forgings ASTM A182, Grade F316L, } \\
\text { Nitronic } 50^{\mathrm{R}} \text {, or } 316 \text { SST Ball and Stem. Seats to be } \\
\text { determined for particular service }\end{array}$ & $\begin{array}{l}\text { ASTM A351 Grade CG6MMN (5) } \\
\text { ASTM A351 Grade CF3M ASTM } \\
\text { A182 Grade F316L See Notes } 1 \& 4 \\
\text { ANSI B16.34 }\end{array}$ \\
\hline Ball Valves & $\begin{array}{l}1 / 2 " \text { and } \\
\text { Larger }\end{array}$ & $\begin{array}{l}\text { 150\# Class, RF, Body, Ball and Stem Nitronic } 50^{\mathrm{R}} \text {, } \\
\text { Casting ASTM A351, Grade CG6MMN or } 316 \mathrm{SST} \text {, } \\
\text { Castings ASTM A351, Grade CF8M Forgings ASTM } \\
\text { A182 Grade F316. Seats to be determined for particular } \\
\text { service. }\end{array}$ & $\begin{array}{l}\text { ASTM A351 Grade ASTM A351 } \\
\text { Grade CF8M ASTM A182 Grade } \\
\text { F316 See notes } \underline{1} \text { \& } 4 \text { ANSI B16.34 }\end{array}$ \\
\hline $\begin{array}{l}\text { Butter-fly } \\
\text { Values }\end{array}$ & $2 "$ and Larger & $\begin{array}{l}\text { 150\# Class, RF or Wafer Type, Body and Disc Nitronic } \\
50^{\mathrm{R}} \text { Casting ASTM A351, Grade CG6MMN or } 316 \text { SST, } \\
\text { Castings Grade CF8M, Forgings ASTM A182, Grade } \\
\text { F316, 17-4 PH Stem, Lever-Lock Handle, Seal Rings to be } \\
\text { determined for Particular service. }\end{array}$ & $\begin{array}{l}\text { ASTM A351 Grade CG6MMN (5) } \\
\text { ASTM A351 Grade CF8M ASTM } \\
\text { A182 Grade F316 ANSI B16.34 } \\
\text { Note } 1\end{array}$ \\
\hline & $1 / 2 "-2^{\prime \prime}$ & $\begin{array}{l}\text { 150\# Class, BW Ends, Body, Cover, and Plug Nitronic } \\
50^{\mathrm{R}} \text {, Casting ASTM A351, Grade CG6MMN or } 316 \text { SST, } \\
\text { Castings ASTM A351, Grade CF3M, Forgings ASTM } \\
\text { A182, Grade F316L, Sleeve Material to be determined for } \\
\text { particular service. }\end{array}$ & $\begin{array}{l}\text { ASTM A351 Grade CG6MMN (5) } \\
\text { ASTM A351 Grade CF3M ASTM } \\
\text { A182 Grade F316L ANSI B16.34 } \\
\text { Note } 1\end{array}$ \\
\hline $\begin{array}{l}\text { Plug } \\
\text { Values }\end{array}$ & $\begin{array}{l}1 / 2^{\prime \prime} \text { and } \\
\text { Larger }\end{array}$ & $\begin{array}{l}\text { 150\# Class, RF, Body, Cover and Plug Nitronic } 50^{\mathrm{R}} \text {, } \\
\text { Castings ASTM A351, Grade CG6MMN or } 316 \mathrm{SST} \text {, } \\
\text { Castings ASTM A351, Grade CF8M, Forgings, ASTM } \\
\text { A182, Grade F316, Sleeve Material to be determined for } \\
\text { particular service. }\end{array}$ & $\begin{array}{l}\text { ASTM A351, Grade CG6MMN (5) } \\
\text { ASTM A351 Grade CF8M, ASTM } \\
\text { A182 Grade F316 ANSI B16.34 } \\
\text { Note } 1\end{array}$ \\
\hline Flanges & All Sizes & $\begin{array}{l}\text { 150\# ANSI B16.5, RF, Weld-neck, Nitronic } 50^{\mathrm{R}} \text { Stainless } \\
\text { Steel }\end{array}$ & $\begin{array}{l}\text { ASTM A351 Grade CG6MMN } \\
\text { ASTM A182 Grade FXM-19 ANSI } \\
\text { B16.5 Note } 3\end{array}$ \\
\hline \multicolumn{2}{|c|}{ Orifice Flanges } & 300\# ANSI B16.5, Raised Face Weld-Neck Type, Nitronic & ASTM A351 Grade CG6MMN \\
\hline
\end{tabular}


PIPING MATERIAL STANDARDS

Stainless Steel Nitronic 50 ${ }^{R}, 150$ psi Service Rating, Line Class AB

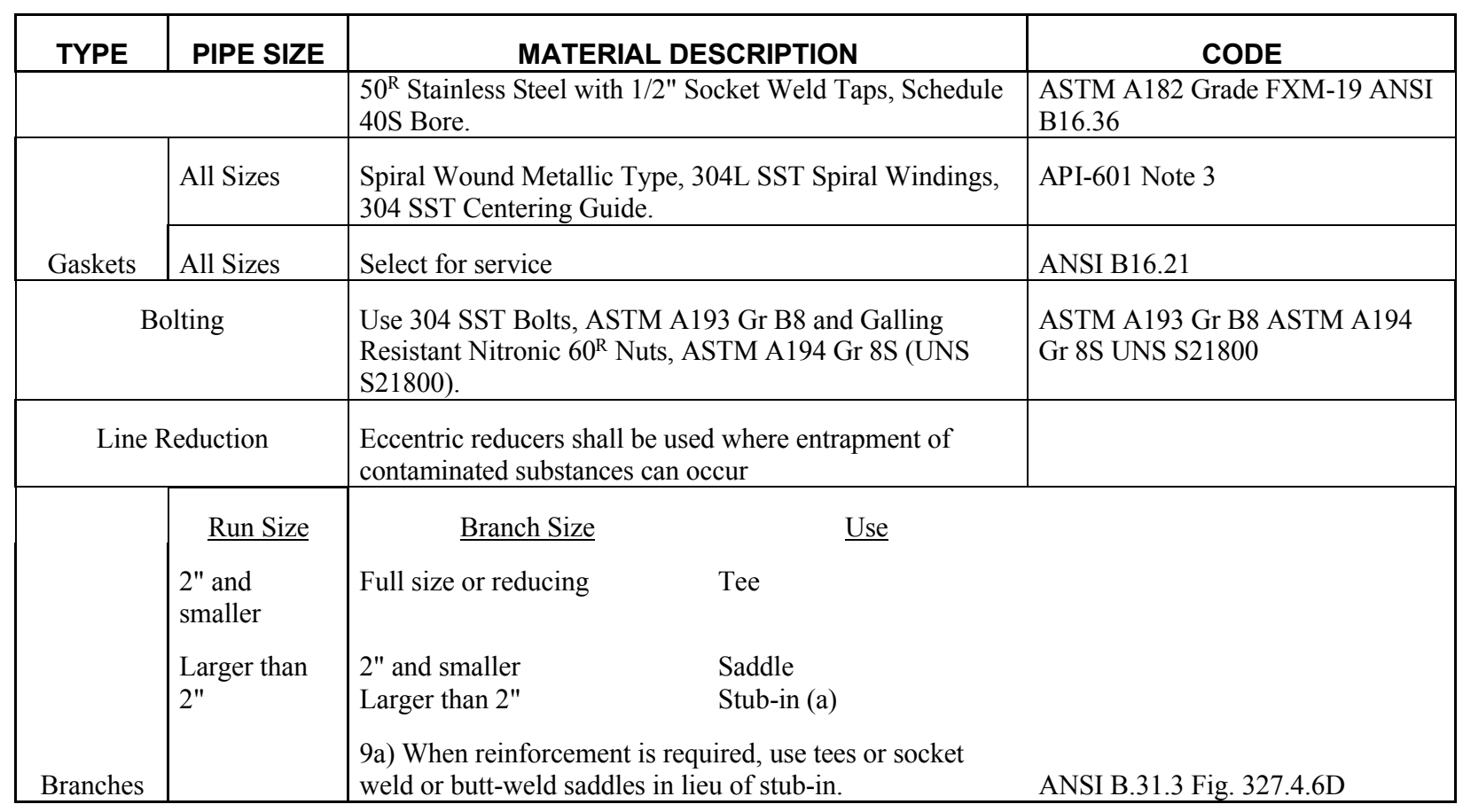




\section{PIPING MATERIAL STANDARDS}

\section{Stainless Steel, 300 psi Service Rating, Line Class AC}

\section{Design Notes}

1. This specification shall be used in lieu of $\mathrm{AD}$ and $\mathrm{AR}$ for high temperature service. Temperature limit $1000^{\circ} \mathrm{F}$.

2. All external components for valves shall be 304,316 , or approved equal SST.

3. Where corrosion will be excessive or higher pressures or other pipe stresses are encountered, the designer has the option of selecting heavier walled pipe and higher rated valves and fittings.

4. When using spiral wound gaskets, see Rules for Bolted Flange Connections, ASME Section VIII Div.1 Appendix 2 or ASME Section VIII Div. 2 Appendix 3 and ASME Section III Div.1, Subsection ND, Section ND-3658 (for equivalent pressure approach) to ensure that the gaskets are applicable for the particular service intended. In cases where spiral wound gaskets are incompatible with the above requirements contact ICPP Design Engineering Organization for further guidance.

5. Full port valves are preferred in fouling services.

6. Alternate disc materials which are suitable for the intended service may be substituted to minimize galling between the seat and disc with prior approval of ICPP Design Engineering Organization.

\begin{tabular}{|c|c|c|c|}
\hline TYPE & PIPE SIZE & MATERIAL DESCRIPTION & CODE \\
\hline & $\begin{array}{l}2 " \text { and } \\
\text { Smaller }\end{array}$ & $\begin{array}{l}\text { Seamless Stainless Steel, ASTM A312, Grade TP347 } \\
\text { Schedule } 80 \text { S, Beveled Ends. }\end{array}$ & ASTM A312 Grade TP347 \\
\hline Pipe & $\begin{array}{l}2-1 / 2 " \text { and } \\
\text { Larger }\end{array}$ & $\begin{array}{l}\text { Seamless Stainless Steel, ASTM A312, Grade TP347, } \\
\text { Schedule 40S, Beveled Ends. }\end{array}$ & ASTM A312 Grade TP347 \\
\hline & $\begin{array}{l}2 " \text { and } \\
\text { smaller }\end{array}$ & $\begin{array}{l}\text { Seamless Wrought Stainless Steel, BW Ends, ASTM A403, } \\
\text { Class WP-S Grade 347, Schedule 80S, Beveled Ends or } \\
\text { Forged Stainless Steel, SW Ends, 6000\#, ASTM A182 } \\
\text { Grade F } 347 .\end{array}$ & $\begin{array}{l}\text { ASTM A403 Class WP-S Grade } \\
347 \text { ASTM A182 Grade F } 347 \\
\text { Dimensions Per ANSI B16.9 or } \\
\text { B16.11 }\end{array}$ \\
\hline Fittings & $\begin{array}{l}2-1 / 2^{\prime \prime} \text { and } \\
\text { Larger }\end{array}$ & $\begin{array}{l}\text { Seamless Wrought Stainless Steel, BWE, ASTM A403 } \\
\text { Class WP-S Grade 347, Schedule 40S Beveled Ends. }\end{array}$ & $\begin{array}{l}\text { ASTM A403 Class WP-S Grade } \\
347 \text { Dimensions per ANSI B16.9 }\end{array}$ \\
\hline \multirow[b]{3}{*}{$\begin{array}{l}\text { Gate } \\
\text { Valves }\end{array}$} & $\begin{array}{l}2 " \text { and } \\
\text { Smaller }\end{array}$ & $\begin{array}{l}\text { 300\# class BW or SW Ends, OS\&Y, Bolted Bonnet, } \\
\text { Integral Seat, Body, Bonnet and Disc } 347 \text { SST, Casting } \\
\text { ASTM A351, Grade CF8C, Forgings ASTM A182, Grade } \\
\text { F347, Packing Grafoil }^{\mathrm{R}} \text {, Bonnet Gasket Grafoil }{ }^{\mathrm{R}} \text {. }\end{array}$ & $\begin{array}{l}\text { ASTM A351 Grade CF8C ASTM } \\
\text { A182 Grade F347 ANSI B16.34 } \\
\text { Note } 2 \& 6\end{array}$ \\
\hline & $\begin{array}{l}2-1 / 2^{\prime \prime} \text { and } \\
\text { Larger }\end{array}$ & 300\# Class, Same as above except BW Ends. & Same as above \\
\hline & $\begin{array}{l}1 / 2^{\prime \prime} \text { and } \\
\text { Larger }\end{array}$ & 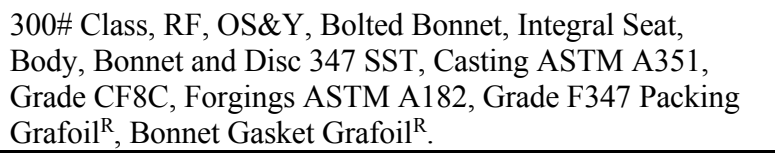 & $\begin{array}{l}\text { ASTM A351 Grade CF8C ASTM } \\
\text { A182 Grade F347 ANSI B16.34 } \\
\text { Note } 2 \& 6\end{array}$ \\
\hline \multirow[b]{2}{*}{$\begin{array}{l}\text { Globe } \\
\text { Valves }\end{array}$} & $\begin{array}{l}2^{\prime \prime} \text { and } \\
\text { Smaller }\end{array}$ & $\begin{array}{l}\text { 300\# Class, BW or SW Ends, OS\&Y, Bolted Bonnet, Body, } \\
\text { Bonnet and Disc } 347 \text { Stainless Steel, Integral Seat, Castings } \\
\text { ASTM A351, Grade CF8C, Forgings ASTM A182, Grade } \\
\text { F347, Grafoil }{ }^{\mathrm{R}} \text { Packing, Bonnet Gasket Grafoil }{ }^{\mathrm{R}} \text {. }\end{array}$ & $\begin{array}{l}\text { ASTM A } 351 \text { Grade CF8C ASTM } \\
\text { A182 Grade F347 ANSI B16.34 } \\
\text { Note } 2 \& 6\end{array}$ \\
\hline & $\begin{array}{l}2-1 / 2 " \text { and } \\
\text { Larger }\end{array}$ & Same as above except for BWE. & Same as above \\
\hline
\end{tabular}


PIPING MATERIAL STANDARDS

347 Stainless Steel, 300 psi Service Rating, Line Class AC

\begin{tabular}{|c|c|c|c|}
\hline TYPE & PIPE SIZE & MATERIAL DESCRIPTION & CODE \\
\hline & $\begin{array}{l}1 / 2^{\prime \prime} \text { and } \\
\text { Larger }\end{array}$ & $\begin{array}{l}\text { 300\# Class, RF, OS\&Y, Bolted Bonnet, Body, Bonnet and } \\
\text { Disc } 347 \text { SST, Integral Seat, Castings ASTM A351, Grade } \\
\text { CF8C, Forgings ASTM A182, Grade F347, Grafoil }{ }^{\mathrm{R}} \\
\text { Packing, Bonnet Gasket Grafoil }^{\mathrm{R}} \text {. }\end{array}$ & $\begin{array}{l}\text { ASTM A351 Grade CF8C ASTM } \\
\text { A182 Grade F347 ANSI B16.34 } \\
\text { Note } 2 \& 6\end{array}$ \\
\hline & $\begin{array}{l}1 / 2 " \text { and } \\
\text { Larger } \\
\text { Bellows- } \\
\text { Seal }\end{array}$ & $\begin{array}{l}\text { 300\# Class, BW Ends, SST Bellows Seal Type, OS\&Y, } \\
\text { Bolted Bonnet, Integral Seat, Body and Bonnet } 347 \\
\text { Stainless Steel, Castings ASTM A351, Grade CF8C, } \\
\text { Forgings ASTM A182, Grade F347, Disc } 316 \text { Stainless } \\
\text { Steel, Packing Grafoil }{ }^{\mathrm{R}} \text {, Bonnet Gasket Grafoil }{ }^{\mathrm{R}} \text {, 3" Long } \\
\text { Minimum Pipe Stub each end. }\end{array}$ & $\begin{array}{l}\text { ASTM A351 Grade CF8C ASTM } \\
\text { A182 Grade F347 ANSI B16.34 } \\
\text { Note } 2 \& 6\end{array}$ \\
\hline & $1 / 2^{\prime \prime}-2^{\prime \prime}$ & $\begin{array}{l}\text { 300\# Class, BW or SW Ends, Swing Type, Bolted Bonnet, } \\
\text { Body, Bonnet and Disc } 347 \text { Stainless Steel, Integral Seat, } \\
\text { Castings ASTM A351, Grade CF8C, Forgings ASTM } \\
\text { A182, Grade F347, Bonnet Gasket Grafoil }{ }^{\mathrm{R}} \text {. }\end{array}$ & $\begin{array}{l}\text { ASTM A351 Grade CF8C ASTM } \\
\text { A182 Grade F347 ANSI B16.34 } \\
\text { Note } 2 \& 6\end{array}$ \\
\hline & $\begin{array}{l}2 " \text { and } \\
\text { Larger }\end{array}$ & Same as above except for BWE. & Same as above \\
\hline $\begin{array}{l}\text { Check } \\
\text { Valves }\end{array}$ & $\begin{array}{l}1 / 2^{\prime \prime} \text { and } \\
\text { Larger }\end{array}$ & $\begin{array}{l}\text { 300\# Class, RF, Swing Type, Bolted Bonnet, Body, Bonnet } \\
\text { and Disc } 347 \text { SST, Integral Seat, Castings ASTM A351, } \\
\text { Grade CF8C, Forgings ASTM A182 Grade F347, Bonnet } \\
\text { Gasket Grafoil }{ }^{\text {. }} \text {. }\end{array}$ & $\begin{array}{l}\text { ASTM A351 Grade CF8C ASTM } \\
\text { A182 Grade F347 ANSI B16.34 } \\
\text { Note } 2 \& 6\end{array}$ \\
\hline \multirow[b]{3}{*}{ Ball Valves } & $1 / 2 "$ - 2" & $\begin{array}{l}\text { 300\# Class, BW or SW Ends, } 3 \text { Piece, Split-Body or Top } \\
\text { Entry, Body, Ball and Stem } 347 \text { Stainless Steel Castings } \\
\text { ASTM A351, Grade CF8C, Forgings ASTM A182, Grade } \\
\text { F347, Seal and Gasket Grafoil }{ }^{\mathrm{R}} \text {, seats to be determined for } \\
\text { particular service. }\end{array}$ & $\begin{array}{l}\text { ASTM A351 Grade CF8C ASTM } \\
\text { A182 Grade F347 See Note } 5 \text { ANSI } \\
\text { B16.34 }\end{array}$ \\
\hline & $\begin{array}{l}2 " \text { and } \\
\text { Larger }\end{array}$ & Same as above except BWE. & Same as above \\
\hline & $\begin{array}{l}1 / 2^{\prime \prime} \text { and } \\
\text { Larger }\end{array}$ & $\begin{array}{l}\text { 300\# Class, RF, Body, Ball and Stem } 347 \text { SST, Castings } \\
\text { ASTM A351, Grade CF8C, Forgings ASTM A182 Grade } \\
\text { F347, Seal and Gasket Grafoil }{ }^{\mathrm{R}} \text {, Seats to be determined for } \\
\text { particular service. }\end{array}$ & $\begin{array}{l}\text { ASTM A351 Grade CF8C ASTM } \\
\text { A182 Grade F347 See Note } 5 \text { ANSI } \\
\text { B16.34 }\end{array}$ \\
\hline \multirow[b]{3}{*}{$\begin{array}{c}\text { Plug } \\
\text { Valves }\end{array}$} & $1 / 2 "$ - 2" & $\begin{array}{l}\text { 300\# Class, BW or SW Ends, Body, Cover, and Plug } 347 \\
\text { SST, Castings ASTM A351, Grade F8C, Forgings ASTM } \\
\text { A182, Grade F347, Sleeve material to be determined for } \\
\text { particular service. }\end{array}$ & $\begin{array}{l}\text { ASTM A351 Grade CF8C ASTM } \\
\text { A182 Grade F347 ANSI B16.34 }\end{array}$ \\
\hline & $\begin{array}{l}2 " \text { and } \\
\text { Larger }\end{array}$ & Same as above except BWE. & Same as above \\
\hline & $\begin{array}{l}1 / 2^{\prime \prime} \text { and } \\
\text { Larger }\end{array}$ & $\begin{array}{l}\text { 300\# Class, RF, Body, Cover and Plug } 347 \text { SST, Castings } \\
\text { ASTM A351, Grade CF8C, Forgings ASTM A182, Grade } \\
\text { F347, Sleeve material to be determined for particular } \\
\text { service. }\end{array}$ & $\begin{array}{l}\text { ASTM A351 Grade CF8C ASTM } \\
\text { A182 Grade F347 ANSI B16.34 }\end{array}$ \\
\hline Flanges & All Sizes & $\begin{array}{l}\text { 300\# ANSI B16.5, RF, Slip-on, Weld-neck, or Lap Joint } \\
\text { and Stub-end, Forged } 347 \text { SST. }\end{array}$ & $\begin{array}{l}\text { ASTM A182 Grade F347 ANSI } \\
\text { B16.5 }\end{array}$ \\
\hline $\begin{array}{l}\text { Orifice } \\
\text { Flanges }\end{array}$ & All Sizes & $\begin{array}{l}\text { 300\# ANSI B16.5, Raised Face Weld-neck Type, Forged } \\
347 \text { Stainless Steel with 1/2" Socket Weld Taps, Schedule } \\
\text { 40S Bore. }\end{array}$ & $\begin{array}{l}\text { ASTM A182 Grade F347 } \\
\text { Dimensions Per ANSI B16.36 }\end{array}$ \\
\hline
\end{tabular}


PIPING MATERIAL STANDARDS

347 Stainless Steel, 300 psi Service Rating, Line Class AC

\begin{tabular}{|c|c|c|c|c|}
\hline TYPE & PIPE SIZE & \multicolumn{2}{|c|}{ MATERIAL DESCRIPTION } & CODE \\
\hline & All Sizes & \multicolumn{2}{|c|}{$\begin{array}{l}\text { Spiral Wound Metallic Type, 304L SST Spiral Windings } \\
\text { with Grafoil }^{\mathrm{R}} \text { Filler, } 304 \text { SST Centering Guide. }\end{array}$} & API-601 Note 4 \\
\hline Gaskets & All Sizes & \multicolumn{2}{|l|}{ Grafoil $^{\mathrm{R}}$} & ANSI B16.21 \\
\hline \multicolumn{2}{|c|}{ Bolting } & \multicolumn{2}{|c|}{$\begin{array}{l}\text { Use } 304 \text { SST Bolts ASTM A193 Gr B8 and Galling } \\
\text { Resistant Nitronic } 60^{\mathrm{R}} \text { Nuts ASTM A194 Gr 8S (UNS } \\
\text { S21800). }\end{array}$} & $\begin{array}{l}\text { ASTM A193 Gr B8 ASTM A194 } \\
\text { Gr 8S UNS S21800 }\end{array}$ \\
\hline \multicolumn{2}{|c|}{ Line Reduction } & \multicolumn{2}{|c|}{$\begin{array}{l}\text { Eccentric Reducers shall be used where entrapment of } \\
\text { contaminated substances can occur. }\end{array}$} & \\
\hline & $\underline{\text { Run Size }}$ & $\underline{\text { Branch Size }}$ & $\underline{\text { Use }}$ & \\
\hline Branches & $\begin{array}{l}2 " \text { and } \\
\text { smaller } \\
\text { Larger than } \\
2^{\prime \prime}\end{array}$ & $\begin{array}{l}\text { Full size or reducing } \\
\text { Full Size } \\
2 " \text { and smaller } \\
\text { Larger than 2" } \\
\text { (a) When reinforcem } \\
\text { or butt-weld saddles i }\end{array}$ & $\begin{array}{l}\text { Tee } \\
\text { Tee } \\
\text { Saddle } \\
\text { Stub-in (a) } \\
\text { quired, use tees or socket weld } \\
\text { stub-in. }\end{array}$ & ANSI B.31.3 Fig. 3327.4.6D \\
\hline
\end{tabular}




\section{PIPING MATERIAL STANDARDS}

\section{Stainless Steel - 304L, 150 - 300 psi Service Rating, Line Class AD}

\section{Design Notes}

1. This specification shall be used instead of Line Class AR when increased corrosion allowance is required.

2. This line class shall be used in lieu of all or portions of the following line classes. See the Piping Material Specification Index and the specific project specification for more details.

o NWCF Spec. SP-453504-50-3 line classes AD, AE and AF.

3. For oxygen service all valves shall be prepared, packaged and tagged "For Oxygen Service".

4. For oxygen service all piping shall be carefully cleaned to be completely free of oil, grease, dirt, or other readily oxidizable foreign material.

5. NPT Fittings shall be used in cell only for instruments and equipment (such as pressure gages, pumps, etc) where welded ends are not available. Out-of-Cell NPT fittings may be used when upstream of the cell wall block valve with prior written approval of ICPP Design Engineering Organization.

6. Three hole flanges, fabricated in accordance with Dwg. 1550-CPP-666-P-600, shall be used where remote removal of equipment or components is required.

\begin{tabular}{|c|l|l|l|}
\hline TYPE & PIPE SIZE & \multicolumn{1}{|c|}{ MATERIAL DESCRIPTION } & \multicolumn{1}{c|}{ CODE } \\
\hline \multirow{2}{*}{ Pipe } & $\begin{array}{l}2 " \& \\
\text { Smaller }\end{array}$ & Seamless 304L SST, Sch 80S, Beveled Ends. & ASTM A312 Grade TP304L \\
\cline { 2 - 4 } & Larger & 304L SST, Sch 40S, Beveled Ends. & ASTM A814 Grade TP304L \\
\hline \multirow{2}{*}{ Fittings } & \begin{tabular}{l} 
Smaller \\
\cline { 2 - 4 }
\end{tabular} & $\begin{array}{l}\text { Leamless Wrought SST, Sch 80S, BW Ends, ASTM A403 } \\
\text { Class WP-S Grade 304L or Forged SST, 6000\#, SW Ends, } \\
\text { ASTM A182. }\end{array}$ & $\begin{array}{l}\text { ASTM A403L Class WP-S Grade } \\
\text { Grade F 304L Dimensions per } \\
\text { ANSI B16.9 or B16.11 }\end{array}$ \\
\cline { 2 - 5 } & & $\begin{array}{l}\text { Seamless Wrought SST, Sch 40S BW Ends. } \\
\text { For remainder of specification see Line Class AR Spec. All } \\
\text { butt-weld valves shall match the bore of pipe and fittings. }\end{array}$ & $\begin{array}{l}\text { ASTM A403 Class WP-S Grade } \\
\text { 304L Dimensions per ANSI B16.9 }\end{array}$ \\
\hline
\end{tabular}


PIPING MATERIAL STANDARDS

\section{Stainless Steel Tubing, 150 - 300 psi Service Rating, Line Class AM}

\section{Design Notes}

1. This specification can be used on original plant process installations where tubing was used (where organics in cells prevent welding of piping components). Note restrictions on use of tubing in 15432.3 of this AE Standard.

2. Where corrosion will be excessive or higher pressures or other stresses are encountered, the designer has the option of selecting thicker walled (.065" wall) tubing.

3. Only 304 SST or 316 SST seats will be acceptable for in-cell valves or on steam valves. No TFE in cell.

4. Swagelok ${ }^{R}$ compression fittings are required without or equal substitution for make-up of all tubing except where components must be compatible with existing flare fittings (Parker Triple-Lok ${ }^{\mathrm{R}}$ ). Parker Triple-Lok ${ }^{\mathrm{R}}$ flare fittings may be used in this case only. The intent is to eliminate all flare fittings where possible. See also1543 2.3.

5. Use tube to pipe weld connectors for fit-up.

6. Alternate disc materials which are suitable for the intended service may be substituted to minimize galling between the seat and disc with prior approval of ICPP Design Engineering Organization.

\begin{tabular}{|c|c|c|c|}
\hline TYPE & PIPE SIZE & MATERIAL DESCRIPTION & CODE \\
\hline Tube & $1 / 8^{\prime \prime}-1 "$ & $\begin{array}{l}\text { Stainless Steel MIL-T-8504 Composition } 304 \text { or ASTM } \\
\text { A269 TP 304, .035" Wall, Seamless. }\end{array}$ & $\begin{array}{l}\text { ASTM A269 TP304 or MIL-T-8504 } \\
\text { Comp. } 304\end{array}$ \\
\hline \multirow[b]{2}{*}{ Fittings } & $1 / 8^{\prime \prime}-1 "$ & $\begin{array}{l}\text { Stainless Steel, ASTM A276 TP316, Swagelok }{ }^{\mathrm{R}} \\
\text { Compression Fittings. }\end{array}$ & ASTM A276 TP 316 \\
\hline & $\begin{array}{l}1 / 8^{\prime \prime} \text { and } \\
\text { Larger }\end{array}$ & Parker Triple Lock ${ }^{\mathrm{R}}$ flare Fittings & See Note 4 \\
\hline \multirow[b]{3}{*}{$\begin{array}{l}\text { Globe } \\
\text { Valves }\end{array}$} & $\begin{array}{l}1 / 8^{\prime \prime}-1 " \\
\text { Bellows- } \\
\text { Seal }\end{array}$ & $\begin{array}{l}\text { 150\# - 300\# Class, Bellows Sealed BW, 316L Stainless } \\
\text { Steel Body, } 316 \text { Stainless Steel Disc, Integral Seat, Grafoil }{ }^{\mathrm{R}} \\
\text { Gasket and Packing. }\end{array}$ & $\begin{array}{l}\text { ASTM A351 Grade CF3M ASTM } \\
\text { A182 Grade F316L Notes } 5 \text { \& } 6\end{array}$ \\
\hline & $1 / 8^{\prime \prime}-1 "$ & $\begin{array}{l}\text { 150\# - 300\# Class, SW, OS\&Y, BB, 316L Stainless Steel } \\
\text { Body and Integral Seat, } 316 \text { SST Disc, Grafoil }{ }^{\mathrm{R}} \text { Gasket and } \\
\text { Packing. }\end{array}$ & Same as above \\
\hline & $\begin{array}{l}3 / 4^{\prime \prime} \\
\text { Bellows } \\
\text { Seal }\end{array}$ & $\begin{array}{l}\text { Nupro }{ }^{\mathrm{R}} 12 \mathrm{U} \text { Series, Pipe Butt Weld Ends, 316L SST Body, } \\
316 \text { SST Stem Insert. For use as cell wall valve. }\end{array}$ & \\
\hline \multirow[t]{2}{*}{ Needle } & Valves & $\begin{array}{l}\text { Stainless Steel Type } 316 \text { forged Body, } 3000 \text { PSI Rating, } \\
\text { Swagelok }{ }^{\mathrm{R}} \text { X Swagelok }{ }^{\mathrm{R}} \text { Ends, Whitey }{ }^{\mathrm{R}} \text {. For out-of-cell } \\
\text { use only. }\end{array}$ & \\
\hline & $1 / 2^{\prime \prime}-1 "$ & $\begin{array}{l}\text { 150\# - 300\# Class, Butt Weld and Socket Weld Ends, } \\
\text { Swing Type, Bolted Cover, 316L Stainless Steel Body, } \\
\text { Integral Seats, } 316 \text { Stainless Steel Disc. }\end{array}$ & $\begin{array}{l}\text { ASTM A351 Grade CF3M ASTM } \\
\text { A182 Grade F316L Notes } 5 \& 6\end{array}$ \\
\hline $\begin{array}{l}\text { Check } \\
\text { Valves }\end{array}$ & $1 / 8^{\prime \prime}-1 "$ & $\begin{array}{l}3000 \#, \text { Swagelok }{ }^{\mathrm{R}} \text { X Swagelok }{ }^{\mathrm{R}} \text { Ends, Spring Return, } 316 \\
\text { SST. For Out-of-Cell Use Only. }\end{array}$ & \\
\hline \multicolumn{2}{|c|}{ Tubing Bends } & Bends shall be minimum 5 times nominal tube diameter. & \\
\hline
\end{tabular}




\section{PIPING MATERIAL STANDARDS}

\section{Stainless Steel - 304L, 150 psi Service Rating, Line Class AR}

\section{Design Notes}

1. This specification at the $150 \#$ service rating will form the bulk of all process and in-cell utility piping at ICPP.

2. Where corrosion will be excessive or higher pressure or other pipe stresses are encountered, the designer has the option of selecting heavier walled pipe and higher rated valves \& fittings. See also line class AD for heavier walled pipe.

3. All external components for valves shall be 304,316 , or approved equal stainless steel.

4. Do not use plug, diaphragm, or butterfly valves for steam service.

5. This line class shall be used in lieu of all or portions of the following line classes. See the Piping Material Specification Line Class List and the specific project specifications for more details.

o NWCF Spec. SP-453504-50-3 line classes AD, AE, and AF.

6. Full port ball valves are preferred in fouling services.

7. When using spiral wound gaskets, see Rules for Bolted Flange Connections, ASME Section VIII Div. 1 Appendix 2 or ASME Section VIII Div. 2 Appendix 3 and ASME Section III Div.1, Subsection ND, Section ND-3658 (for equivalent pressure approach) to ensure that the gaskets are applicable for the particular service intended. In cases where spiral wound gaskets are incompatible with the above requirements contact ICPP Design Engineering Organization for further guidance.

8. FPR Line Class AF (304L SST Pipe Sch 10) shall be considered Line Class AR for future installations. Line Class AS in FPR shall be considered Line Class AR for future installations but shall be upgraded to: (Accountability sample bias control governs this wall thickness).

1/2" Pipe Sch 160

3/4" Pipe Sch 80

9. Material of construction for cell wall block valve bodies on utility lines not contacting process solutions going into cells can be 316 SST or 304 SST instead of 316L SST. (ASTM A351 Grade CF8M or Grade CF8 ASTM A182 Grade F 316 or Grade F 304)

10. Note that in some portions of the original plant process installations, tubing was used. Organics in these in-cell areas may prevent welding on piping components. See line class AM for these cases.

11. For oxygen service all valves shall be prepared, packaged, and tagged "For Oxygen Service."

12. For oxygen service all piping shall be carefully cleaned to be completely free of oil, grease, dirt, or other readily oxidizable foreign material.

13. When ball valves are to be used for cryogenic services use only valves designed specifically for cryogenic service.

14. Examples: R-Con ${ }^{\mathrm{R}}$ International R-Con ${ }^{\mathrm{R}}$ connector, Swing-bolt type.

15. Fittings and equipment in this line class for firewater lines or raw water lines supplying fire water mains shall be specified per NFPA-13 and Factory Mutual Data Sheet 2-8N for above ground piping and NFPA 24 and Factory Mutual Data Sheet 3-10 for underground piping in lieu of the fittings and equipment specified herein. 


\section{PIPING MATERIAL STANDARDS}

\section{Stainless Steel - 304L, 150 psi Service Rating, Line Class AR}

16. NPT fittings shall be used in-cell only for instruments and equipment (such as pressure gages, pumps, valve actuators etc.) where weld ends are not normally available. Out-of-Cell NPT fittings may be used when upstream of the cell-wall block valve with prior written approval of ICPP Design Engineering Organization.

17. Where required diaphragm valves shall be oriented to allow for free draining.

18. Three hole flanges, fabricated in accordance with Dwg. 1550-CPP-666-P-600, shall be used where remote removal of equipment or components is required.

19. Alternate disc materials which are suitable for the intended service may be substituted to minimize galling between the seat and disc with prior approval of ICPP Design Engineering Organization.

\begin{tabular}{|c|c|c|c|}
\hline TYPE & PIPE SIZE & MATERIAL DESCRIPTION & CODE \\
\hline \multirow[b]{2}{*}{ Pipe } & $1 / 4 "-2-1 / 2 "$ & $\begin{array}{l}\text { Seamless Stainless Steel, ASTM A } 312 \text { Grade TP304L, Sch. } \\
40 \text { S, Beveled Ends for BW, Plain Ends for SW, or } \\
\text { Screwed. }\end{array}$ & ASTM A312 TP304L \\
\hline & 3" - 12" & $\begin{array}{l}\text { Stainless Steel, ASTM A814 Grade TP304L, Sch. 40, } \\
\text { Beveled Ends for BW, Plain Ends for SW or Screwed. }\end{array}$ & ASTM A814 TP304L \\
\hline \multirow[b]{2}{*}{ Fittings } & $\begin{array}{l}2 " \text { and } \\
\text { Smaller }\end{array}$ & $\begin{array}{l}\text { Seamless Wrought SST, BW Ends, Sch 40S, ASTM A403 } \\
\text { Class WP-S Grade 304L or Forged SST, SW Ends, 3000\#, } \\
\text { ASTM A } 182 \text { Grade F304L }\end{array}$ & $\begin{array}{l}\text { ASTM A403 Class WP-S Grade } \\
\text { 304L ASTM A182 Grade F 304L } \\
\text { Dimensions per ANSI B16.9 or } \\
\text { B16.11 }\end{array}$ \\
\hline & $\begin{array}{l}2-1 / 2 " \text { and } \\
\text { Larger }\end{array}$ & Seamless Wrought SST, BWE, Sch 40S. & $\begin{array}{l}\text { ASTM A403 Class WP-S Grade } \\
\text { 304L Dimensions per ANSI B16.9 }\end{array}$ \\
\hline \multirow[b]{3}{*}{$\begin{array}{l}\text { Gate } \\
\text { Valves }\end{array}$} & $\begin{array}{l}2 " \text { and } \\
\text { Smaller }\end{array}$ & $\begin{array}{l}\text { 150\# Class, BW or SW Ends, OS\&Y, Bolted Bonnet, } \\
\text { Integral Seat, Body and Bonnet 316L SST, Casting ASTM } \\
\text { A351, Grade CF3M Forgings ASTM A182, Grade F316L } \\
\text { Packing Grafoil }^{\mathrm{R}} \text {, Bonnet Gasket Grafoil }{ }^{\mathrm{R}} \text { Disc } 316 \text { SST. }\end{array}$ & $\begin{array}{l}\text { ASTM A351 Grade CF3M ASTM } \\
\text { A182 Grade F316L ANSI B16.34 } \\
\text { See Notes } 3,9, \& 19\end{array}$ \\
\hline & $\begin{array}{l}2-1 / 2 " \text { and } \\
\text { Larger }\end{array}$ & 150\# Class, Same as above except BW Ends. & Same as above \\
\hline & $\begin{array}{l}1 / 2 \text { and } \\
\text { Larger }\end{array}$ & $\begin{array}{l}\text { 150\# Class, RF, OS\&Y, Bolted Bonnet, Integral Seat, Body } \\
\text { and Bonnet } 316 \text { SST Casting ASTM A351, Grade CF8M, } \\
\text { Forgings ASTM A182, Grade F316, Packing Grafoil }^{\mathrm{R}} \\
\text { Bonnet Gasket Grafoil }^{\mathrm{R}} \text {, Disc } 316 \text { SST. }\end{array}$ & $\begin{array}{l}\text { ASTM A351 Grade CF8M ASTM } \\
\text { A182 Grade F316 See Notes 3, 7, } 9 \text {, } \\
\text { \& } 19 \text { ANSI B16.34 }\end{array}$ \\
\hline \multirow[b]{3}{*}{$\begin{array}{l}\text { Globe } \\
\text { Valves }\end{array}$} & $\begin{array}{l}2 " \text { and } \\
\text { Smaller }\end{array}$ & $\begin{array}{l}\text { 150\# Class, BW or SW Ends, OS\&Y, Bolted Bonnet, Body } \\
\text { and Bonnet 316L Stainless Steel, Integral Seat, Castings } \\
\text { ASTM A351, Grade CF3M, Forgings ASTM A182, Grade } \\
\text { F316L, 316 SST Disc, Grafoil }{ }^{\mathrm{R}} \text { Packing, Bonnet Gasket } \\
\text { Grafoil }^{\mathrm{R}} \text {. }\end{array}$ & $\begin{array}{l}\text { ASTM A351 Grade CF3M ASTM } \\
\text { A182 Grade F316L See Notes 3, } 9 \text {, } \\
\& 19 \\
\text { ANSI B16.34 }\end{array}$ \\
\hline & $\begin{array}{l}2-1 / 2 " \text { and } \\
\text { Larger }\end{array}$ & Same as above except for BWE & Same as above \\
\hline & $\begin{array}{l}1 / 2^{\prime \prime} \text { and } \\
\text { Larger }\end{array}$ & $\begin{array}{l}\text { 150\# Class, RF, OS\&Y, Bolted Bonnet, Body and Bonnet } \\
316 \text { SST, Integral Seat, Castings ASTM A351, Grade } \\
\text { CF8M, Forgings ASTM A182, Grade F316, } 316 \text { SST Disc, } \\
\text { Grafoil }^{\mathrm{R}} \text { Packing, Bonnet Gasket Grafoil }{ }^{\mathrm{R}} \text {. }\end{array}$ & $\begin{array}{l}\text { ASTM A351 Grade CF8M ASTM } \\
\text { A182 Grade F316 See Notes 3, 7, 9, } \\
\& 19 \text { ANSI B16.34 }\end{array}$ \\
\hline
\end{tabular}


PIPING MATERIAL STANDARDS

Stainless Steel - 304L, 150 psi Service Rating, Line Class AR

\begin{tabular}{|c|c|c|c|}
\hline TYPE & PIPE SIZE & MATERIAL DESCRIPTION & CODE \\
\hline & $\begin{array}{l}1 / 2 " \text { and } \\
\text { Larger } \\
\text { Bellows } \\
\text { Seal }\end{array}$ & $\begin{array}{l}\text { 150\# Class, BW Ends, SST Bellows Seal Type, OS\&Y } \\
\text { Bolted Bonnet, Integral Seat, Body and Bonnet 316L } \\
\text { Stainless Steel, Castings ASTM A351, Grade CF3M, } \\
\text { Forgings ASTM A182, Grade F316L, Disc 316 Stainless } \\
\text { Steel, Grafoil }{ }^{\mathrm{R}} \text { Packing, Bonnet Gasket Grafoil }{ }^{\mathrm{R}} \text { 3" Long } \\
\text { Minimum Pipe Stub Each End. }\end{array}$ & $\begin{array}{l}\text { ASTM A351 Grade CF3M ASTM } \\
\text { A182 Grade F316L See Notes 3, } 9 \\
\& 19 \\
\text { ANSI B16.34 }\end{array}$ \\
\hline \multirow[b]{3}{*}{$\begin{array}{l}\text { Check } \\
\text { Valves }\end{array}$} & 1/2" - 2" & $\begin{array}{l}\text { 150\# Class, BW or SW Ends, Swing Type, Bolted Bonnet, } \\
\text { Body and Bonnet 316L Stainless Steel, Integral Seat, } \\
\text { Castings ASTM A351, Grade CF3M, Forgings ASTM } \\
\text { A182 Grade F316L, Bonnet Gasket Grafoil }{ }^{\mathrm{R}} \text {. Disc } 316 \\
\text { Stainless Steel. }\end{array}$ & $\begin{array}{l}\text { ASTM A351 Grade CF3M ASTM } \\
\text { A182 Grade F316L See Notes 3, } 9 \text {, } \\
\& 19 \\
\text { ANSI B16.34 }\end{array}$ \\
\hline & $\begin{array}{l}2 " \text { and } \\
\text { Larger }\end{array}$ & Same as above except for BWE. & Same as above \\
\hline & $\begin{array}{l}1 / 2 " \text { and } \\
\text { Larger }\end{array}$ & $\begin{array}{l}\text { 150\# Class, RF, Swing Type, Bolted Bonnet, Body and } \\
\text { Bonnet 316 SST, Integral Seat, castings ASTM A351, } \\
\text { Grade CF8M, Forgings ASTM A182, Grade F316, Bonnet } \\
\text { Gasket Grafoil }{ }^{\mathrm{R}} \text {, Disc } 316 \text { SST. }\end{array}$ & $\begin{array}{l}\text { ASTM A351 Grade CF8M ASTM } \\
\text { A182 Grade F316 Dimensions per } \\
\text { ANSI B16.34 See Notes } 3,7,9, \& \\
19\end{array}$ \\
\hline \multirow[b]{3}{*}{ Ball Valves } & 1/2" - 2" & $\begin{array}{l}\text { 150\# Class, BW or SW Ends, } 3 \text { Piece, Split Body or Top } \\
\text { Entry, Body 316L Stainless Steel, Castings ASTM A351, } \\
\text { Grade CF3M, Forgings ASTM A182, Grade F316L, 316 } \\
\text { SST Ball and stem, Seal and Gasket Grafoil }{ }^{\mathrm{R}} \text {, Seats to be } \\
\text { determined for particular service. }\end{array}$ & $\begin{array}{l}\text { ASTM A351 Grade CF3M ASTM } \\
\text { A182 Grade F316L See Notes 3, } 6 \text {, } \\
\text { 13, \& } 9 \text { ANSI B16.34 }\end{array}$ \\
\hline & $\begin{array}{l}2 " \text { and } \\
\text { Larger }\end{array}$ & Same as above except BWE. & Same as above \\
\hline & $\begin{array}{l}1 / 2 " \text { and } \\
\text { Larger }\end{array}$ & $\begin{array}{l}\text { 150\# Class, RF, Body, Ball and Stem } 316 \text { SST, Castings } \\
\text { ASTM A351, Grade CF8M, Forgings ASTM A182 Grade } \\
\text { F316, Seal and Gasket Grafoil, Seats to be determined for } \\
\text { particular service. }\end{array}$ & $\begin{array}{l}\text { ASTM A351 Grade CF8M ASTM } \\
\text { A182 Grade F316 See Notes 3, } 6 \text {, } \\
\text { 13, \& } 9 \text { ANSI B16.34 }\end{array}$ \\
\hline $\begin{array}{c}\text { Butterfly } \\
\text { Valves }\end{array}$ & $2 "$ and larger & $\begin{array}{l}\text { 150\# Class, RF, or Wafer Type Body and Disc 316 SST, } \\
\text { Castings ASTM A351, Grade CF8M, Forgings ASTM } \\
\text { A182, Grade F316, 17-4 PH Stem, Lever-Lock Handle, } \\
\text { Seal Rings to be determined for particular service. }\end{array}$ & $\begin{array}{l}\text { ASTM A351 Grade CF8M ASTM } \\
\text { A182 Grade F316 ANSI B16.34 See } \\
\text { Notes } 3,4,7, \& 9\end{array}$ \\
\hline \multirow[b]{3}{*}{$\begin{array}{c}\text { Plug } \\
\text { Valves }\end{array}$} & 1/2" - 2" & $\begin{array}{l}\text { 150\# Class, BW or SW Ends, Body, Cover, and Plug 316L } \\
\text { SST, Castings ASTM A351, Grade CF3M, Forgings ASTM } \\
\text { A182, Grade F316L, Sleeve Material to be determined for } \\
\text { particular service. }\end{array}$ & $\begin{array}{l}\text { ASTM A351 Grade CF3M ASTM } \\
\text { A182 Grade F316L See Notes 3, } 4 \\
\& 9 \\
\text { ANSI B16.34 }\end{array}$ \\
\hline & $\begin{array}{l}2 " \text { and } \\
\text { Larger }\end{array}$ & Same as above except BWE. & Same as above \\
\hline & $\begin{array}{l}1 / 2 " \text { and } \\
\text { Larger }\end{array}$ & $\begin{array}{l}\text { 150\# Class, RF, Body, Cover, and Plug } 316 \text { SST, Castings } \\
\text { ASTM A351, Grade CF8M, Forgings, ASTM A182, Grade } \\
\text { F316, Sleeve Material to be determined for particular } \\
\text { service. }\end{array}$ & $\begin{array}{l}\text { ASTM A351 Grade CF8M ASTM } \\
\text { A182 Grade F316 ANSI B16.34 See } \\
\text { Notes } 3,4,7, \& 9\end{array}$ \\
\hline Diaphragm & 1/2" - 2" & $\begin{array}{l}\text { 150\# Class, BW or SW Ends, Body and Cover 316L SST, } \\
\text { Castings ASTM A351, Grade CF3M, Forgings ASTM } \\
\text { A182, Grade F316L, Diaphragm Material and packing to be } \\
\text { determined for particular service. }\end{array}$ & $\begin{array}{l}\text { ASTM A351 Grade CF3M ASTM } \\
\text { A182 Grade F316L ANSI B16.34 } \\
\text { See Notes } 3,4,9, \& 17\end{array}$ \\
\hline
\end{tabular}


PIPING MATERIAL STANDARDS

Stainless Steel - 304L, 150 psi Service Rating, Line Class AR

\begin{tabular}{|c|c|c|c|c|}
\hline TYPE & PIPE SIZE & \multicolumn{2}{|c|}{ MATERIAL DESCRIPTION } & CODE \\
\hline & $\begin{array}{l}2 " \text { and } \\
\text { Larger }\end{array}$ & \multicolumn{2}{|c|}{ Same as above except BWE. } & Same as above \\
\hline & $\begin{array}{l}1 / 2^{\prime \prime} \text { and } \\
\text { Larger }\end{array}$ & \multicolumn{2}{|c|}{$\begin{array}{l}\text { 150\# Class, RF, Body and Cover } 316 \text { SST, Castings ASTM } \\
\text { A351, Grade CF8M, Forgings ASTM A182, Grade F316, } \\
\text { Diaphragm and Packing Material to be determined for } \\
\text { particular service. }\end{array}$} & $\begin{array}{l}\text { ASTM A351 Grade CF8M ASTM } \\
\text { A182 Grade F316 ANSI B16.34 See } \\
\text { Notes } 3,4,7, \& 17\end{array}$ \\
\hline $\begin{array}{l}\text { Remote } \\
\text { Connectors }\end{array}$ & All Sizes & \multicolumn{2}{|c|}{$\begin{array}{l}\text { 150\# Class, Remotely Operated R-Con }{ }^{\mathrm{R}} \text { Type Connector, } \\
\text { 304L or } 316 \mathrm{~L} \text { Stainless Steel Butt Weld Hubs, } 316 \text { Stainless } \\
\text { Steel Seal Rings and Clamps, Nitronic } 60^{\mathrm{R}} \text { Screw, Schedule } \\
\text { 40S Bore. }\end{array}$} & Note 14 \\
\hline Flanges & All Sizes & \multicolumn{2}{|c|}{$\begin{array}{l}\text { 150\# ANSI B16.5, RF, Slip-On, Weld Neck, or Lap Joint } \\
\text { and Stub-End Forged 304L SST. }\end{array}$} & $\begin{array}{l}\text { ASTM A182 Grade F304L Note } 7 \\
\& 18 \text { ANSI B16.5 }\end{array}$ \\
\hline $\begin{array}{l}\text { Orifice } \\
\text { Flanges }\end{array}$ & All Sizes & \multicolumn{2}{|c|}{$\begin{array}{l}\text { 300\# ANSI B16.5, Raised Face Weld-Neck, Type, Forged } \\
\text { Stainless Steel with } 1 / 2 \text { " Socket Weld Taps, Schedule } 40 \text { S } \\
\text { Bore. }\end{array}$} & $\begin{array}{l}\text { ASTM A182 Grade F304L ANSI } \\
\text { B16.36 }\end{array}$ \\
\hline & All Sizes & \multicolumn{2}{|c|}{$\begin{array}{l}\text { Spiral Wound Metallic Type, 304L SST Spiral Windings } \\
\text { with Grafoil }^{\mathrm{R}} \text { Filler, } 304 \text { SST Centering Guide. }\end{array}$} & $\begin{array}{l}\text { ASTM A182 Grade F304L API-601 } \\
\text { Note } 7\end{array}$ \\
\hline Gaskets & All Sizes & \multicolumn{2}{|l|}{ Grafoil $^{\mathrm{R}}$} & ANSI B16.21 \\
\hline \multicolumn{2}{|c|}{ Bolting } & \multicolumn{2}{|c|}{$\begin{array}{l}\text { Use } 304 \text { SST Bolts ASTM A } 193 \text { Gr B8 and Galling } \\
\text { Resistant Nitronic } 60^{\mathrm{R}} \text { Nuts ASTM A194 Gr 8S (UNS } \\
\text { S21800). }\end{array}$} & $\begin{array}{l}\text { ASTM A193 Gr B8 ASTM A194 } \\
\text { GR 8S UNS S21800 }\end{array}$ \\
\hline \multicolumn{2}{|c|}{ Line Reduction } & \multicolumn{2}{|c|}{$\begin{array}{l}\text { Eccentric Reducers Shall be used where entrapment of } \\
\text { contaminated substances can occur }\end{array}$} & \\
\hline \multirow{3}{*}{ Branches } & $\underline{\text { Run Size }}$ & \multirow{2}{*}{$\begin{array}{l}\text { Branch Size } \\
\text { Full size or reducing } \\
\text { Full size } \\
\text { 2" and smaller } \\
\text { Larger than 2" }\end{array}$} & $\underline{\text { Use }}$ & \\
\hline & $\begin{array}{l}\text { Run Size 2" } \\
\text { and smaller } \\
\text { Larger than } \\
2^{\prime \prime}\end{array}$ & & $\begin{array}{l}\text { Tee } \\
\text { Tee } \\
\text { Saddle } \\
\text { Stub-in (a) }\end{array}$ & ANSI B.31.3 Fig. $327.4 .6 \mathrm{D}$ \\
\hline & & \multicolumn{2}{|c|}{$\begin{array}{l}\text { (a) When reinforcement is required use tees or socket-weld } \\
\text { or butt-weld saddles in lieu of stub-in. }\end{array}$} & \\
\hline
\end{tabular}


PIPING MATERIAL STANDARDS

\section{Carpenter $20 \mathrm{Cb}^{\mathrm{R}}$ Stainless Steel, 150 psi Service Rating, Line Class CA}

\section{Design Notes}

1. Examples: R-Con ${ }^{\mathrm{R}}$ Connector, R-Con ${ }^{\mathrm{R}}$ International, Swing-bolt Type.

2. Plug valves shall not be used in steam service.

3. Three hole flanges, fabricated in accordance with Dwg. 1550-CPP-666-P-600 shall be used where remote removal of equipment or components is required.

4. All external components for valves shall be 304, 316, or approved equal SST.

5. When using spiral wound gaskets, See Rules for Bolted Flange Connections, ASME Section VIII Div. 1 Appendix 2 or ASME Section VIII Div. 2 Appendix 3 and ASME Section III Div.1, Subsection ND Section ND-3658 (for equivalent pressure approach) to ensure that the gaskets are applicable for the particular service intended. In cases where spiral wound gaskets are incompatible with the above requirements contact ICPP Design Engineering Organization for further guidance.

6. Pipe stub wall thickness shall be the same as connecting pipe.

7. Alternate disc materials which are suitable for the intended service may be substituted to minimize galling between the seat and disc with prior approval of the ICPP Design Engineering Organization.

\begin{tabular}{|c|c|c|c|}
\hline TYPE & PIPE SIZE & MATERIAL DESCRIPTION & CODE \\
\hline \multirow[b]{2}{*}{ Pipe } & $1 / 4 "$ - 3/4" & $\begin{array}{l}\text { Carpenter } 20 \mathrm{Cb}-3^{\mathrm{R}} \text {, Seamless SCH } 80 \mathrm{~S} \\
\text { UNS NO8020 }\end{array}$ & ASTM 729 \\
\hline & $\begin{array}{l}1 " \text { and } \\
\text { Larger }\end{array}$ & Carpenter $20 \mathrm{Cb}-3^{\mathrm{R}}$, Seamless SCH $40 \mathrm{~S}$. & ASTM 729 UNS NO8020 \\
\hline \multirow[b]{2}{*}{ Fittings } & $1 / 4^{\prime \prime}-3 / 4^{\prime \prime}$ & Carpenter $20 \mathrm{Cb}-3^{\mathrm{R}}, \mathrm{BW}, \mathrm{SCH} 80 \mathrm{~S}$ & ASTM B366 Grade WP20CB \\
\hline & $\begin{array}{l}1 " \text { and } \\
\text { Larger }\end{array}$ & Carpenter $20 \mathrm{Cb}-3^{\mathrm{R}}, \mathrm{BW}, \mathrm{SCH} 40 \mathrm{~S}$. & Same as above \\
\hline \multirow[b]{2}{*}{$\begin{array}{l}\text { Gate } \\
\text { Valves }\end{array}$} & All Sizes & $\begin{array}{l}150 \# \text { Class, BW, OS\&Y, BB, Carpenter } 20 \mathrm{Cb}-3^{\mathrm{R}} \text { Body, } \\
\text { Bonnet and Disc, Integral Seat Grafoil }{ }^{\mathrm{R}} \text { Packing and } \\
\text { Gasket. }\end{array}$ & $\begin{array}{l}\text { ASTM B462 UNS NO8020 ASTM } \\
\text { A351 Grade CN7M ANSI B16.34 } \\
\text { See Notes } 4,6, \& 7\end{array}$ \\
\hline & $\begin{array}{l}1 / 2^{\prime \prime} \text { and } \\
\text { Larger }\end{array}$ & $\begin{array}{l}\text { 150\# Class, RF, OS\&Y, BB, Carp. } 20 \text { Cb- } 3^{\mathrm{R}} \text { Body, Bonnet } \\
\text { and Disc, Integral Seat, Grafoil }{ }^{\mathrm{R}} \text { Packing and Gasket. }\end{array}$ & See Notes $4,5, \& 7$ \\
\hline \multirow[b]{3}{*}{$\begin{array}{l}\text { Globe } \\
\text { Valves }\end{array}$} & All Sizes & $\begin{array}{l}\text { 150\#, Class, BW, OS\&Y, BB Carp. } 20 \mathrm{Cb}-3^{\mathrm{R}} \text { Body, Bonnet } \\
\text { and Disc, Integral Seat, Grafoil }{ }^{\mathrm{R}} \text { Packing and Gasket. }\end{array}$ & $\begin{array}{l}\text { Same as above } \\
\text { ANSI B16.34 See Notes } \\
4,6, \& 7\end{array}$ \\
\hline & $\begin{array}{l}1 / 2 " \text { and } \\
\text { Larger } \\
\text { Bellows- } \\
\text { Seal }\end{array}$ & $\begin{array}{l}\text { 150\# Class, BW, Bellows Sealed OS\&Y, BB, Carp. } 20 \\
\text { Cb- } 3^{\mathrm{R}} \text { Body, Bonnet and Disc, Integral Seat, and Bellows, } \\
\text { Grafoil }^{\mathrm{R}} \text { Gasket. Shall be provided with factory welded } 3 \\
\text { inch long Carpenter } 20 \mathrm{Cb}-3^{\mathrm{R}} \text { pipe stub on each end. }\end{array}$ & Same as above \\
\hline & $\begin{array}{l}1 / 2^{\prime \prime} \text { and } \\
\text { Larger }\end{array}$ & $\begin{array}{l}\text { 150\# Class, RF, OS\&Y, BB, Carp. } 20 \mathrm{Cb}-3^{\mathrm{R}} \text { Body, Bonnet } \\
\text { and Disc, and Integral Seat, Grafoil }{ }^{\mathrm{R}} \text { Gasket. }\end{array}$ & $\begin{array}{l}\text { Same as above } \\
\text { ANSI B16.34 See Notes } 4,5, \& 7\end{array}$ \\
\hline $\begin{array}{l}\text { Check } \\
\text { Valves }\end{array}$ & All Sizes & $\begin{array}{l}\text { 150\# Class, BW, Swing Type, Carp. } 20 \mathrm{Cb}-3^{\mathrm{R}} \text { Body, } \\
\text { Bonnet and Disc, Integral Seat, Grafoil }{ }^{\mathrm{R}} \text { Gasket. }\end{array}$ & $\begin{array}{l}\text { Same as above } \\
\text { ANSI B16.34 See Notes } 4,6, \& 7\end{array}$ \\
\hline
\end{tabular}


PIPING MATERIAL STANDARDS

\section{Carpenter $20 \mathrm{Cb}^{\mathrm{R}}$ Stainless Steel, 150 psi Service Rating, Line Class CA}

\begin{tabular}{|c|c|c|c|}
\hline TYPE & PIPE SIZE & MATERIAL DESCRIPTION & CODE \\
\hline & $\begin{array}{l}1 / 2 " \text { and } \\
\text { Larger }\end{array}$ & $\begin{array}{l}\text { 150\# Class, RF, Wafer Check, Carp. } 20 \text { Cb-3 } 3^{\mathrm{R}} \text { Body, Trim } \\
\text { and Plates, Metal to Metal Seal, S/S Spring. }\end{array}$ & ANSI 16.34 See Notes $4 \& 5$ \\
\hline \multirow[b]{2}{*}{ Ball Valves } & All Sizes & $\begin{array}{l}\text { 150\# Class, BW, Carpenter } 20 \mathrm{Cb}-3^{\mathrm{R}} \text { Body, Ball and Stem, } \\
\text { Lever Operated. Seats and Body Seals to be determined for } \\
\text { particular service. }\end{array}$ & $\begin{array}{l}\text { Same as above } \\
\text { ANSI B16.34 See Notes } 4 \& 6\end{array}$ \\
\hline & $\begin{array}{l}1 / 2 " \text { and } \\
\text { Larger }\end{array}$ & $\begin{array}{l}150 \# \text { Class, RF, Carp. } 20 \mathrm{Cb}-3^{\mathrm{R}} \text { Body, Ball and Stem, Lever } \\
\text { Operated. Seats and Seals to be Determined for particular } \\
\text { service. }\end{array}$ & $\begin{array}{l}\text { ASTM B462 UNS NO8020 Grade } \\
\text { CN7M UNS NO802 ANSI B16.34 } \\
\text { See Notes } 4 \text { \& } 5\end{array}$ \\
\hline $\begin{array}{l}\text { Butterfly } \\
\text { Valves }\end{array}$ & All Sizes & $\begin{array}{l}\text { 150\# Class, Single Flanged Lug Type, Carp. } 20 \text { Cb- } 3^{\mathrm{R}} \text { Body } \\
\text { and Disc, } 17-4 \text { pH Stem, Grafoil }{ }^{\mathrm{R}} \text { Seal Rings, Grafoil }{ }^{\mathrm{R}} \\
\text { Packing. }\end{array}$ & $\begin{array}{l}\text { Same as above } \\
\text { ANSI B16.34 See Notes } 4 \& 5\end{array}$ \\
\hline \multirow[b]{2}{*}{$\begin{array}{l}\text { Plug } \\
\text { Valves }\end{array}$} & All Sizes & $\begin{array}{l}\text { 150\# Class, BW, Carp. } 20 \mathrm{Cb}-3^{\mathrm{R}} \text { Body, Cover, and Plug, } \\
\text { Wrench Operated, } 4 " \text { and Larger Gear Operated. Sleeve and } \\
\text { Diaphragm Material to be determined for particular service. }\end{array}$ & $\begin{array}{l}\text { Same as above } \\
\text { ANSI B16.34 See Notes } 2,4, \& 6\end{array}$ \\
\hline & $\begin{array}{l}1 / 2 " \text { and } \\
\text { Larger }\end{array}$ & $\begin{array}{l}150 \# \text { Class, RF, Carp. } 20 \mathrm{Cb}-3^{\mathrm{R}} \text { Body, Cover, and Plug, } 4 " \\
\text { and Larger Gear Operated. Sleeve and Diaphragm Material } \\
\text { to be determined for particular service. }\end{array}$ & $\begin{array}{l}\text { Same as above } \\
\text { ANSI B16.34 See Notes } 2,4, \& 5\end{array}$ \\
\hline \multirow[b]{2}{*}{$\begin{array}{l}\text { Diaphragm } \\
\text { Valves }\end{array}$} & All Sizes & $\begin{array}{l}\text { 150\# Class, BW, Carp. } 20 \mathrm{Cb}-3^{\mathrm{R}} \text { Body, Bonnet, and Stem, } \\
\text { Weir Type, Diaphragm Material to be determined for } \\
\text { particular service. }\end{array}$ & ANSI B16.34 See Notes 4 \& 6 \\
\hline & $\begin{array}{l}1 / 2^{\prime \prime} \text { and } \\
\text { Larger }\end{array}$ & $\begin{array}{l}\text { 150\# Class, RF, Carp. } 20 \mathrm{Cb}-3^{\mathrm{R}} \text { Body, Bonnet and Stem, } \\
\text { Weir Type, Diaphragm Material to be determined for } \\
\text { particular service. }\end{array}$ & ANSI B16.34 See Notes $4 \& 5$ \\
\hline \multicolumn{2}{|c|}{ Remote Connectors } & $\begin{array}{l}\text { 150\# Class, Remotely Operated R-Con }{ }^{\mathrm{R}} \text { Type Connector, } \\
\text { Carpenter Cb- } 3^{\mathrm{R}} \text { Butt Weld Hubs and Seal Rings, } 316 \\
\text { Stainless Steel Clamps, Nitronic } 60^{\mathrm{R}} \text { Screw. }\end{array}$ & See Note 1 \\
\hline \multicolumn{2}{|c|}{ Flanges } & $\begin{array}{l}\text { 150\# ANSI B16.5, Carp. } 20 \text { Cb-3 } 3^{\mathrm{R}} \text {, RF, Weld neck or Lap } \\
\text { Joint and Stub End. }\end{array}$ & $\begin{array}{l}\text { ASTM B462 UNS NO8020 ANSI } \\
\text { B16.5 See Note } 3\end{array}$ \\
\hline \multicolumn{2}{|c|}{ Orifice Flanges } & $\begin{array}{l}\text { 300\# ANSI Raised Face Weld-neck Type, Forged, } \\
\text { Carpenter } 20 \mathrm{Cb}-3^{\mathrm{R}} \text {, ASTM B } 462 \text { with 1/2" SCRD Taps. }\end{array}$ & $\begin{array}{l}\text { ASTM B462 UNS NO8020 ANSI } \\
\text { B16.36 }\end{array}$ \\
\hline \multirow[b]{2}{*}{ Gaskets } & & Grafoil $^{\mathrm{R}}$ Flange Gaskets, Flat Ring, 1/16" Thick. & ANSI B16.21 \\
\hline & All Sizes & $\begin{array}{l}\text { Spiral Wound Metallic Type, Carpenter } 20 \mathrm{Cb}-3^{\mathrm{R}} \text { Spiral } \\
\text { Windings with Grafoil }^{\mathrm{R}} \text { Filler, } 304 \text { SST Centering Guide. }\end{array}$ & $\begin{array}{l}\text { ASTM B } 366 \text { Grade WP 20CB API- } \\
601 \text { Note } 5\end{array}$ \\
\hline \multicolumn{2}{|c|}{ Bolting } & $\begin{array}{l}\text { Use } 304 \text { SST Bolts ASTM A } 193 \text { Gr B8 and Galling } \\
\text { Resistant Nitronic } 60^{\mathrm{R}} \text { Nuts ASTM A194 Gr 8S (UNS } \\
\text { S21800). }\end{array}$ & $\begin{array}{l}\text { ASTM A193 Gr B8 ASTM A } 194 \\
\text { Gr 8S UNS S } 21800\end{array}$ \\
\hline Branches & All Sizes & Use full or reducing tees. & \\
\hline \multicolumn{2}{|c|}{ Line Reductions } & \multicolumn{2}{|l|}{$\begin{array}{l}\text { Use reducers. Eccentric reducers shall be used where } \\
\text { entrapment of contaminated substances can occur. }\end{array}$} \\
\hline
\end{tabular}


PIPING MATERIAL STANDARDS

\section{Hastelloy $C-4^{R}$ or $C-22^{R}, 150$ psi Service Rating, Line Class HC}

\section{Design Notes}

1. These pipe wall thicknesses are suggested only. The designer shall choose specific pipe wall thicknesses for the particular corrosion service and pressure rating and other piping stresses.

2. Due to lack of availability of C-4 and increased corrosion resistance, Hastelloy C- $22^{\mathrm{R}}$ shall replace C-4 where applicable.

$$
\begin{aligned}
& \text { Hastelloy C-4 }{ }^{\mathrm{R}} \text { (UNS NO6455) } \\
& \text { Hastelloy C-22 }
\end{aligned}
$$

3. Examples: R-Con International R-Con ${ }^{\mathrm{R}}$ Connector, swing-bolt type.

4. NDE requirements on all line class HC systems shall be $100 \%$ radiography on all butt weld joints and $100 \%$ LP examination on all socket weld joints.

\begin{tabular}{|c|c|c|c|}
\hline TYPE & PIPE SIZE & MATERIAL DESCRIPTION & CODE \\
\hline \multirow[b]{3}{*}{ Pipe } & $1 / 8^{\prime \prime}-3 / 4^{\prime \prime}$ & $\begin{array}{l}\text { Hastelloy C- } 4^{\mathrm{R}} \text { or C-22R } 2^{\mathrm{R}} \text { Solution Annealed Beveled Ends } \\
\text { SCH } 160 \text { Seamless. }\end{array}$ & $\begin{array}{l}\text { ASTM B622 UNS NO6455 UNS } \\
\text { NO6022 }\end{array}$ \\
\hline & $1^{\prime \prime}-2^{\prime \prime}$ & $\begin{array}{l}\text { Hastelloy C- } 4^{\mathrm{R}} \text { or C- } 22^{\mathrm{R}} \text { Solution Annealed Beveled Ends } \\
\text { SCH } 80 \text { Seamless. }\end{array}$ & $\begin{array}{l}\text { ASTM B622 UNS NO6455 UNS } \\
\text { NO6022 }\end{array}$ \\
\hline & $\begin{array}{l}2-1 / 2 " \text { and } \\
\text { Larger }\end{array}$ & $\begin{array}{l}\text { Hastelloy C- } 4^{\mathrm{R}} \text { or C- } 22^{\mathrm{R}} \text { Solution Annealed Beveled Ends } \\
\text { SCH } 40 \text { Seamless. }\end{array}$ & $\begin{array}{l}\text { ASTM B } 622 \text { UNS NO6455 UNS } \\
\text { NO6022 }\end{array}$ \\
\hline \multicolumn{2}{|c|}{ Orifice Flanges } & $\begin{array}{l}\text { 300\# ANSI B16.5, RF, Hastelloy } \mathrm{C}-4^{\mathrm{R}} \text { or } \mathrm{C}-22^{\mathrm{R}} \text { Weld Neck } \\
\text { Type to Match Pipe Schedule with Socket Weld Taps. } \\
\text { Dimensions per ANSI B16.5. }\end{array}$ & $\begin{array}{l}\text { ASTM B574 UNS NO6455 UNS } \\
\text { NO6022 ASTM B575 UNS N06455 } \\
\text { UNS N06022 ANSI B16.36 } \\
\end{array}$ \\
\hline \multicolumn{2}{|c|}{ Gaskets } & Grafoil $^{\mathrm{R}}$ Flange Gasket, Flat Ring 1/16" & ANSI B16.21 \\
\hline \multicolumn{2}{|c|}{ Bolting } & $\begin{array}{l}\text { Use } 304 \text { SST Bolts ASTM A } 193 \text { Gr B8 and Galling } \\
\text { Resistant Nitronic } 60^{\mathrm{R}} \text { Nuts ASTM A194 Gr 8S (UNS } \\
\text { S21800). }\end{array}$ & $\begin{array}{l}\text { ASTM A193 Gr B8 ASTM A194 } \\
\text { GR 8S UNS S21800 }\end{array}$ \\
\hline \multicolumn{2}{|c|}{ Branches } & Use full or reducing tees or saddles. & \\
\hline \multirow[b]{3}{*}{ Fittings } & $1 / 8^{\prime \prime}-3 / 4 "$ & $\begin{array}{l}\text { Seamless Hastelloy C-4 } 4^{\mathrm{R}} \text { or C-22 } 2^{\mathrm{R}} \text { solution Annealed Butt } \\
\text { Welded Ends, Sch } 160 \text {. }\end{array}$ & $\begin{array}{l}\text { ASTM B366 WPHC4S UNS } \\
\text { NO6455 WPHC22S UNS NO6022 } \\
\text { Dimensions per ANSI B16.9 }\end{array}$ \\
\hline & $1^{\prime \prime}-2^{\prime \prime}$ & $\begin{array}{l}\text { Seamless Hastelloy C- } 4^{\mathrm{R}} \text { or C- } 22^{\mathrm{R}} \text { Solution Annealed Butt } \\
\text { Welded Ends, Sch } 80 \text {. }\end{array}$ & Same as above \\
\hline & $\begin{array}{l}2-1 / 2 " \text { and } \\
\text { Larger }\end{array}$ & $\begin{array}{l}\text { Seamless Hastelloy C- } 4^{\mathrm{R}} \text { or C- } 22^{\mathrm{R}} \text { Solution Annealed Butt } \\
\text { Weld Ends, Sch } 40 .\end{array}$ & Same as above \\
\hline \multirow[t]{2}{*}{$\begin{array}{c}\text { Gate } \\
\text { Valves } \\
\end{array}$} & $\begin{array}{l}1 / 2^{\prime \prime} \text { and } \\
\text { Larger }\end{array}$ & $\begin{array}{l}\text { 150\# Class, BW to Match Schedule of Pipe, OS\&Y, BB, } \\
\text { Hastelloy C- } 4^{\mathrm{R}} \text { or C- } 22^{\mathrm{R}} \text { Body, Bonnet and Disc, Integral } \\
\text { Seat, Grafoil }{ }^{\mathrm{R}} \text { Packing and Gasket. }\end{array}$ & $\begin{array}{l}\text { Same as above } \\
\text { ANSI B16.34 Note } 5\end{array}$ \\
\hline & $\begin{array}{l}\text { Same as } \\
\text { above } \\
\square\end{array}$ & $\begin{array}{l}\text { 150\# Class, RF, OS\&Y BB, Hastelloy } \mathrm{C}-4^{\mathrm{R}} \text { or } \mathrm{C}-22^{\mathrm{R}} \text { Body, } \\
\text { Bonnet and Disc, Integral Seat, Grafoil }{ }^{\mathrm{R}} \text { Packing and } \\
\text { Gasket. }\end{array}$ & $\begin{array}{l}\text { Same as above } \\
\text { ANSI B16.34 Note } 5\end{array}$ \\
\hline
\end{tabular}

5. All external components for valves shall be 304,316 or approved equal stainless steel. 
PIPING MATERIAL STANDARDS

Hastelloy C-4 ${ }^{R}$ or C-22 ${ }^{R}$, 150 psi Service Rating, Line Class HC

\begin{tabular}{|c|c|c|c|}
\hline TYPE & PIPE SIZE & MATERIAL DESCRIPTION & CODE \\
\hline \multirow[b]{3}{*}{$\begin{array}{l}\text { Globe } \\
\text { Valves }\end{array}$} & $\begin{array}{l}1 / 2^{\prime \prime} \text { and } \\
\text { Larger }\end{array}$ & $\begin{array}{l}\text { 150\# Class, BW to match Schedule of Pipe, OS\&Y, BB, } \\
\text { Bellows Seal, Hastelloy C- } 4^{\mathrm{R}} \text { or C- } 22^{\mathrm{R}} \text { Body, Bonnet, Disc } \\
\text { and Bellows, Grafoil }{ }^{\mathrm{R}} \text { Gasket, Integral Seat. }\end{array}$ & $\begin{array}{l}\text { Same as above } \\
\text { ANSI B16.34 Note } 5\end{array}$ \\
\hline & $\begin{array}{l}\text { Same as } \\
\text { above } \\
\square\end{array}$ & $\begin{array}{l}\text { 150\# Class, BW to Match Schedule of OS\&Y, BB, } \\
\text { Hastelloy C- } 4^{\mathrm{R}} \text { or C }-22^{\mathrm{R}} \text { Body, Bonnet and Disc, Integral } \\
\text { Seat, Grafoil }{ }^{\mathrm{R}} \text { Packing and Gasket. }\end{array}$ & $\begin{array}{l}\text { Same as above } \\
\text { ANSI B16.34 Note } 5\end{array}$ \\
\hline & $\begin{array}{l}\text { Same as } \\
\text { above } \\
\square\end{array}$ & $\begin{array}{l}150 \# \text { Class, RF, OS\&Y, BB, Hastelloy } \mathrm{C}-4^{\mathrm{R}} \text { or } \mathrm{C}-22^{\mathrm{R}} \text { Body, } \\
\text { Bonnet and Disc, Integral Seat, Grafoil }{ }^{\mathrm{R}} \text { Packing and } \\
\text { Gasket. }\end{array}$ & $\begin{array}{l}\text { Same as above } \\
\text { ANSI B16.34 Note } 5\end{array}$ \\
\hline \multirow[b]{2}{*}{$\begin{array}{l}\text { Check } \\
\text { Valves }\end{array}$} & $\begin{array}{l}1 / 2^{\prime \prime} \text { and } \\
\text { Larger }\end{array}$ & $\begin{array}{l}\text { 150\# Class, BB, BW to match Schedule of Pipe, Hastelloy } \\
\text { C- } 4^{\mathrm{R}} \text { or } \mathrm{C}-22^{\mathrm{R}} \text { Body, Bonnet and Disc, Grafoil }{ }^{\mathrm{R}} \text { Gasket. }\end{array}$ & $\begin{array}{l}\text { Same as above } \\
\text { ANSI B16.34 Note } 5\end{array}$ \\
\hline & $\begin{array}{l}\text { Same as } \\
\text { above } \\
\square\end{array}$ & $\begin{array}{l}\text { 150\# Class, RF, BB, Hastelloy C- } 4^{\mathrm{R}} \text { or } \mathrm{C}-22^{\mathrm{R}} \text { Body, Bonnet } \\
\text { and Disc, Grafoil }{ }^{\mathrm{R}} \text { Gasket. }\end{array}$ & $\begin{array}{l}\text { Same as above } \\
\text { ANSI B16.34 Note } 5\end{array}$ \\
\hline \multirow[b]{2}{*}{ Ball Valves } & $\begin{array}{l}1 / 2 " \text { and } \\
\text { Larger }\end{array}$ & $\begin{array}{l}150 \# \text { Class, BW to match Schedule of Pipe, Hastelloy C- } 4^{\mathrm{R}} \\
\text { or C- } 22^{\mathrm{R}} \text { Body, Ball and Stem, Lever Operated, Seats and } \\
\text { Seals to be determined for particular service. }\end{array}$ & $\begin{array}{l}\text { ASTM B366 WPHC4S UNS } \\
\text { NO6455 ASTM B366 WPHC22S } \\
\text { UNS NO6022 ANSI B16.34 Note } 5 \\
\end{array}$ \\
\hline & $\begin{array}{l}1 / 2^{\prime \prime} \text { and } \\
\text { Larger }\end{array}$ & $\begin{array}{l}\text { 150\# Class, RF, Hastelloy C- } 4^{\mathrm{R}} \text { or C }-22^{\mathrm{R}} \text {, Body, Stem and } \\
\text { Ball Lever Operated, Seats and Seals to be determined for } \\
\text { particular service. }\end{array}$ & Same as above \\
\hline \multicolumn{2}{|c|}{ Remote Connections } & $\begin{array}{l}\text { 150\#, Remotely operated R-Con }{ }^{\mathrm{R}} \text { Type Connector, } \\
\text { Hastelloy C- } 4^{\mathrm{R}} \text { or C- }-22^{\mathrm{R}} \text { Butt Weld Hubs and Seal Rings, } \\
316 \text { or } 304 \text { Stainless Steel Clamps, Nitronic } 60^{\mathrm{R}} \text { Screw. }\end{array}$ & $\begin{array}{l}\text { ASTM B } 574 \text { UNS NO6455 UNS } \\
\text { NO6022 Note } 3\end{array}$ \\
\hline \multirow{2}{*}{\multicolumn{2}{|c|}{ Flanges }} & $\begin{array}{l}\text { 150\# ANSI B16.5, Hastelloy C- } 4^{\mathrm{R}} \text { or C- } 22^{\mathrm{R}} \text { Stub End for } \\
\text { Use w/Slip-on Flanges to make a Lap Joint or Van Stone } \\
\text { Flanged Connection to Vessels, Valves, Pumps, or other } \\
\text { Flanged Equipment. }\end{array}$ & $\begin{array}{l}\text { ASTM B575 UNS NO6455 UNS } \\
\text { NO6022 ASTM B574 UNS } \\
\text { NO6455 UNS NO6022 Dimensions } \\
\text { per ANSI B16.5 }\end{array}$ \\
\hline & & $\begin{array}{l}150 \# \text { ANSI B16.5, Hastelloy C- } 4^{\mathrm{R}} \text { or } \mathrm{C}-22^{\mathrm{R}} \mathrm{RF} \text {, Weld Neck } \\
\text { to match Pipe Schedule. }\end{array}$ & $\begin{array}{l}\text { ASTM B575 UNS NO6455 UNS } \\
\text { NO6022 ASTM B574 UNS } \\
\text { NO6455 UNS NO6022 Dimensions } \\
\text { per ANSI B16.5 }\end{array}$ \\
\hline
\end{tabular}




\section{PIPING MATERIAL STANDARDS}

\section{Hastelloy G-30R, 150 psi Service Rating, Line Class HG}

\section{Design Notes}

1. These pipe wall thicknesses are suggested only. The designer shall choose specific pipe wall thicknesses for the particular corrosion service, piping stresses, and pressure rating.

2. Note that original installation on LET\&D project used Hastelloy G-30R welded seam pipe 1/2" thru 2-1/2" per ASTM B619 class II with 20\% minimum cold reduction and eddy-current testing and 3" thru 8" pipe per ASTM B619 Class I with eddy-current testing. Seamless pipe per ASTM B622 is now available up to 4".

3. Eddy-current testing or $100 \%$ weld $x$-ray required.

4. Liquid penetrant test on root and final pass of welds is required. $100 \% \mathrm{x}$-ray test on final weld is required.

5. Note that original installation on LET\&D project allowed either WPHG30S fittings made from ASTM B622 seamless pipe or WPHG30WX fittings made from ASTM B619 Class I welded seam pipe for 3" to 8" pipe. For future installations all fittings 1/4" to 4" shall be WPHG30S made from ASTM B622 seamless pipe.

6. Flange surfaces shall be finished to 125 microinches AARH for seating to spiral wound gaskets.

7. This gasket for use at LET\&D where concentrated nitric acid is encountered and radiation levels are negligible. For radiation service consult ICPP Design Engineering Organization for a more suitable material. When using spiral wound gaskets, see Rules for Bolted Flange Connections, ASME Section VIII Div. 1 Appendix 2 or ASME Section VIII Div 2 Appendix 3 and ASME Section III Div.1, Subsection ND, Section ND-3658 (for equivalent pressure approach) to ensure that the gaskets are applicable for the particular service intended. In cases where spiral wound gaskets are incompatible with the above requirements contact ICPP Design Engineering Organization for further guidance.

8. All external components for valves shall be 304,316 , or approved equal stainless steel.

9. Note that $316 \mathrm{~L} \mathrm{SST}$ and Carpenter $20 \mathrm{Cb}-3^{\mathrm{R}}$ ball valves were installed where the corrosion service allowed it as original equipment at LET\&D due to lack of availability of Hastelloy G-30 ${ }^{\mathrm{R}}$ valves. For all future work Hastelloy $\mathrm{G}-30^{\mathrm{R}}$ is required unless it can be demonstrated that the above materials are adequate for the particular corrosion service. Prior written approval is required from ICPP Design Engineering Organization.

\begin{tabular}{|c|c|c|c|}
\hline TYPE & PIPE SIZE & MATERIAL DESCRIPTION & CODE \\
\hline \multirow[b]{4}{*}{ Pipe } & $1 / 4^{\prime \prime}$ & $\begin{array}{l}\text { Hastelloy G-30 } 0^{\mathrm{R}} \text {, ASTM B622 UNS NO6030, Seamless, } \\
\text { Sch. } 80 \text { S, Heat Treated and Descaled. }\end{array}$ & ASTM B622 UNS NO6030 \\
\hline & $1 / 2 "$ - 4" & $\begin{array}{l}\text { Hastelloy G-30 } 0^{\mathrm{R}} \text {, ASTM B622 UNS NO6030 Seamless, } \\
\text { Sch. 40S, Heat Treated and Descaled. }\end{array}$ & ASTM B622 UNS NO6030 \\
\hline & $5 "-8 "$ & $\begin{array}{l}\text { Hastelloy G-30 }{ }^{\mathrm{R}} \text {, ASTM B619 UNS NO6030 Class I, } \\
\text { Welded Seam, Sch. 40S, Heat Treated and Descaled. }\end{array}$ & $\begin{array}{l}\text { ASTM B } 619 \text { UNS NO6030 See } \\
\text { Note } 3\end{array}$ \\
\hline & $10^{\prime \prime}-14 "$ & $\begin{array}{l}\text { Hastelloy G-30R }{ }^{\mathrm{R}} \text {, UNS NO6030, Welded Seam from ASTM } \\
\text { B582 material using Hastelloy G-30 } 0^{\mathrm{R}} \text { Weld Filler Material } \\
\text { Sch. 10S, Heated Treated and Descaled. Outside diameter } \\
\text { (O.D.) shall be within the Requirements of ASTM A530 } \\
\text { Table I "Permissible Variations of Outside Diameters" of } \\
\text { the O.D.'s Specified in ANSI B36.19. }\end{array}$ & $\begin{array}{l}\text { ASTM B582 UNS NO6030 See } \\
\text { Note } 4 \\
\text { ASTM A530 ASTM B36.19 }\end{array}$ \\
\hline Fittings & $1 / 4^{\prime \prime}$ & $\begin{array}{l}\text { Hastelloy G-30R }{ }^{\mathrm{R}} \text {, UNS NO6030, ASTM B366, Class } \\
\text { WPHG30S from ASTM B } 622 \text { Seamless Pipe, Sch. 80S, } \\
\text { Butt Weld Ends. }\end{array}$ & $\begin{array}{l}\text { ASTM B366 Class WPHG30S UNS } \\
\text { NO6030 ASTM B622 Dimensions } \\
\text { per ANSI B16.9 }\end{array}$ \\
\hline
\end{tabular}


PIPING MATERIAL STANDARDS

Hastelloy G-30R, 150 psi Service Rating, Line Class HG

\begin{tabular}{|c|c|c|c|}
\hline TYPE & PIPE SIZE & MATERIAL DESCRIPTION & CODE \\
\hline & $1 / 2^{\prime \prime}-4^{\prime \prime}$ & $\begin{array}{l}\text { Hastelloy G-30R }{ }^{\mathrm{R}} \text {, UNS NO6030, ASTM B366 Class } \\
\text { WPHG30S from ASTM B622 Seamless Pipe, Sch. 40S Butt } \\
\text { Weld Ends. }\end{array}$ & Same as above \\
\hline & $5^{\prime \prime}-8 "$ & $\begin{array}{l}\text { Hastelloy G-30 } 0^{\mathrm{R}} \text {, UNS NO6030, ASTM B } 366 \text { Class, } \\
\text { WPHG30WX from ASTM B619 Class I Welded Seam } \\
\text { Pipe, Sch. 40S, Butt Weld Ends. }\end{array}$ & $\begin{array}{l}\text { ASTM B366 Class WPHG30WX } \\
\text { UNS NO6030 ASTM B } 619 \\
\text { Dimensions per ANSI B16.9 }\end{array}$ \\
\hline & $10^{\prime \prime}-14 "$ & $\begin{array}{l}\text { Hastelloy G-30 } 0^{\mathrm{R}} \text {, UNS NO6030, ASTM B366 Class } \\
\text { WPHG30WX from ASTM B582 Material, Sch 10S, Butt } \\
\text { Weld Ends. }\end{array}$ & $\begin{array}{l}\text { ASTM B366 Class WPHG30WX } \\
\text { UNS NO6030 ASTM B582 } \\
\text { Dimensions per ANSI B16.9 }\end{array}$ \\
\hline & All Sizes & $\begin{array}{l}\text { Spiral Wound Metallic Type, Hastelloy G- } 30^{\mathrm{R}} \text { Spiral } \\
\text { Windings with TFE Filler, } 304 \text { SST Centering Guide. }\end{array}$ & $\begin{array}{l}\text { API-601 ASTM B582 UNS } \\
\text { NO6030 See Note } 7\end{array}$ \\
\hline Gaskets & All Sizes & Grafoil $^{\mathrm{R}}$ & ANSI B16.21 \\
\hline & Iting & $\begin{array}{l}\text { Use } 304 \text { SST Bolts ASTM A193 GR B8 and Galling } \\
\text { Resistant Nitronic } 60^{\mathrm{R}} \text { Nuts ASTM A194 GR 8S (UNS } \\
\text { S21800). }\end{array}$ & $\begin{array}{l}\text { ASTM A193 GR B8 ASTM A194 } \\
\text { GR 8S UNS S21800 }\end{array}$ \\
\hline $\begin{array}{l}\text { Gate } \\
\text { Valves }\end{array}$ & All Sizes & $\begin{array}{l}\text { 150\# Class R.F. Bonneted Knife Gate Bi-Direction, Close } \\
\text { Bonnet and Body Fabricated (not cast) Carpenter } 20 \mathrm{Cb}-3^{\mathrm{R}} \text {, } \\
\text { Internal Gate Wiper Between Body and Bonnet and } \\
\text { Multiple Rows of Stem Packing. Viton }{ }^{\mathrm{R}} \text { Steel Belted Radial } \\
\text { Seats. }\end{array}$ & $\begin{array}{l}\text { ASTM B462 UNS-08020 ASTM } \\
\text { B463 UNS-08020 ASTM B472 } \\
\text { UNS-08020 ANSI B16.34 See } \\
\text { Notes } 6 \text { \& } 8\end{array}$ \\
\hline $\begin{array}{l}\text { Globe } \\
\text { Valves }\end{array}$ & All Sizes & $\begin{array}{l}\text { 150\# Class, Body, Bonnet and Disc Fabricated (not case) } \\
\text { Carpenter } 20 \mathrm{Cb}-3^{\mathrm{R}} \text {, Flanged or B.W., Seats and Seals Shall } \\
\text { be determined for the service intended. }\end{array}$ & $\begin{array}{l}\text { ASTM B462 UNS-08020 ASTM } \\
\text { B463 UNS-08020 ASTM B472 } \\
\text { UNS-08020 See Notes } 6 \& 8\end{array}$ \\
\hline \multicolumn{2}{|c|}{ Ball Valves } & & $\begin{array}{l}\text { ASTM B581 UNS-NO6030 ASTM } \\
\text { B462 or } 472 \text { ANSI B16.34 Notes } 8 \\
\& 9\end{array}$ \\
\hline
\end{tabular}


PIPING MATERIAL STANDARDS

\section{PVC Pressure Pipe, Line NA Class 200}

\section{Design Notes}

1. To be used for underground pressurized fire water lines only.

2. Installation shall be per manufacturer's instructions.

3. PVC pipe shall be bedded all around with 4" to 6" of sand.

4. This line class shall not be used in contact with or within 10' of 300 series stainless steel line classes to avoid chloride contamination.

\begin{tabular}{|c|l|l|l|}
\hline TYPE & PIPE SIZE & \multicolumn{1}{|c|}{ MATERIAL DESCRIPTION } & \multicolumn{1}{c|}{ CODE } \\
\hline Pipe & $4 "-12 "$ & $\begin{array}{l}\text { Polyvinyl Chloride (PVC) Pressure Pipe, Class 200, Bell } \\
\text { and Spigot Ends. As manufactured by J-M Manufacturing } \\
\text { Co. Inc. Blue Brute }{ }^{\mathrm{R}} \text { or equal. }\end{array}$ & $\begin{array}{l}\text { AWWA C900 UL Listed and } \\
\text { Factory Mutual approved for fire } \\
\text { protection service. }\end{array}$ \\
\hline Fittings & $4 "-12^{\prime \prime}$ & Ductile Iron per Line Class NR. & \\
\hline
\end{tabular}


PIPING MATERIAL STANDARDS

\section{Carbon Steel - ECTFE Lined (HarlarR), 150 psi Service Rating, Line Class NB}

\section{Design Notes}

1. Manufacturer's suggested bolt torque shall be used in assembling flange joints.

2. No gaskets are normally required between flanges with molded raised faces. Before disconnecting flanges scribe match marks in flange sides to ensure exact line-up when reconnected.

3. Threaded flanges are to be used only on field fit spool pieces to make final connections to flanged pipe.

\begin{tabular}{|c|l|l|l|}
\hline TYPE & PIPE SIZE & \multicolumn{1}{|c|}{ MATERIAL DESCRIPTION } & \multicolumn{1}{c|}{ CODE } \\
\hline Pipe & $1 "-10^{\prime \prime}$ & $\begin{array}{l}\text { Carbon Steel Sch. 40 with ECTFE lining, 150\# ANSI B16.5 } \\
\text { wrought steel Van Stone (lap joint) flanged ends with } \\
\text { molded raised face. }\end{array}$ & $\begin{array}{l}\text { ASTM A587 ASTM A234 ANSI } \\
\text { B16.5 }\end{array}$ \\
\hline Fittings & $1 "-10 "$ & $\begin{array}{l}\text { Wrought steel Van Stone (lap joint) flanged fittings, 150\# } \\
\text { ANSI B16.5 lined with ECTFE, molded raised face. }\end{array}$ & ASTM A234 ANSI B16.5 \\
\hline $\begin{array}{c}\text { Flanges } \\
\text { Blind } \\
\text { Flanges \& } \\
\begin{array}{c}\text { Reducing } \\
\text { Flanges }\end{array}\end{array}$ & $1 "-10 "$ & $\begin{array}{l}\text { Forged Carbon Steel Van Stone (lap joint) and Threaded } \\
\text { Flanges with ECTFE Lining, 150\# ANSI B16.5, Molded } \\
\text { Raised Face. }\end{array}$ & $\begin{array}{l}\text { ASTM A105 or A181 ANSI B16.5 } \\
\text { See Note 3 }\end{array}$ \\
\hline $\begin{array}{c}\text { Plug } \\
\text { Valves }\end{array}$ & All Sizes & $\begin{array}{l}\text { 150\# Class Molded Raised Face Flanged Cast Steel with } \\
\text { ECTFE Lining, PFA Coated Plug (or other coating suitable } \\
\text { for service intended). }\end{array}$ & $\begin{array}{l}\text { ASTM A216 Grade WCB ANSI } \\
\text { B16.34 }\end{array}$ \\
\hline $\begin{array}{c}\text { 3-Way } \\
\text { Plug }\end{array}$ & All Sizes & $\begin{array}{l}\text { 150\# Class Molded Raised Face Flanged, Cast Steel or } \\
\text { Ductile Iron with ECTFE Lining, PTFE Coated Plug (or } \\
\text { other coating suitable for intended service). }\end{array}$ & ASTM A395 or A216 Grade WCB \\
\hline \multicolumn{2}{|c|}{ Bolting } & Hexhead Machine Bolt with Hex Nut, Chrome-moly Steel. & $\begin{array}{l}\text { ASTM A193 Grade B7 ASTM } \\
\text { A194 Grade 2H }\end{array}$ \\
\hline \multicolumn{2}{|c|}{ Branches } & Use Full or Reducing ECTFE Lined Flanged Fittings. & \\
\hline
\end{tabular}


PIPING MATERIAL STANDARDS

\section{Carbon Steel-Weld Fittings, 150 psi Service Rating, Line Class NC}

\section{Design Notes}

1. Line Class NC at FPR included only socket-weld fittings. This line class shall be used for future work.

\begin{tabular}{|c|c|c|c|c|}
\hline TYPE & PIPE SIZE & \multicolumn{2}{|c|}{ MATERIAL DESCRIPTION } & CODE \\
\hline Pipe & All Sizes & \multicolumn{2}{|c|}{ Seamless Carbon Steel, Sch. 40, Plain Ends. } & ASTM A106 Grade B \\
\hline & $\begin{array}{l}2 " \& \\
\text { Smaller }\end{array}$ & \multicolumn{2}{|c|}{$\begin{array}{l}\text { 3000\# Carbon Steel, Forged, Socket-Weld or Butt Weld } \\
\text { Ends. }\end{array}$} & ASTM A105 \\
\hline Fittings & $\begin{array}{l}2-1 / 2 " \text { and } \\
\text { Larger }\end{array}$ & \multicolumn{2}{|c|}{ Seamless Wrought Carbon Steel, Butt Weld Ends. } & ASTM A234 Grade WPB \\
\hline & All Sizes & \multicolumn{2}{|c|}{$\begin{array}{l}\text { 800\# Class, Forged Steel, SW or BW, OS\&Y, BB, Grafoil } \\
\text { Packing \& Gaskets, Trim for Steam Service. }\end{array}$} & ASTM A105 ANSI B16.34 \\
\hline & $\begin{array}{l}\text { Same as } \\
\text { above } \\
\square\end{array}$ & \multicolumn{2}{|c|}{$\begin{array}{l}\text { 150\# Class, Cast Steel, BW, OS\&Y, BB, Standard Trim, } \\
\text { Grafoil }^{\mathrm{R}} \text { Packing and Gaskets. }\end{array}$} & ASTM A216 ANSI B16.34 \\
\hline $\begin{array}{c}\text { Gate } \\
\text { Valves }\end{array}$ & 3" \& Larger & \multicolumn{2}{|c|}{$\begin{array}{l}\text { 150\# Class, Cast Steel, Flanged OS\&Y, BB, STD Trim, } \\
\text { Grafoil }^{\mathrm{R}} \text { Packing and Gaskets. }\end{array}$} & Same as above \\
\hline & All Sizes & \multicolumn{2}{|c|}{$\begin{array}{l}\text { 800\# Class, Forged Steel, SW or BW, OS\&Y, BB, } \\
\text { Renewable Disc \& Seat, Grafoil }{ }^{\mathrm{R}} \text { Packing and Gasket, Trim } \\
\text { for Steam Service. }\end{array}$} & ASTM A105 ANSI B16.34 \\
\hline & $\begin{array}{l}\text { Same as } \\
\text { above } \\
\square\end{array}$ & \multicolumn{2}{|c|}{$\begin{array}{l}\text { 150\# Class, Cast Steel, BW, OS\&Y, BB, Standard Trim, } \\
\text { Grafoil }^{\mathrm{R}} \text { Packing and Gaskets. }\end{array}$} & ASTM A216 ANSI B16.34 \\
\hline $\begin{array}{l}\text { Globe } \\
\text { Valves }\end{array}$ & 3" \& Larger & \multicolumn{2}{|c|}{$\begin{array}{l}\text { 150\# Class, Cast Steel, Flanged, OS\&Y, BB, Standard } \\
\text { Trim, Grafoil }{ }^{\mathrm{R}} \text { Packing and Gaskets. }\end{array}$} & Same as above \\
\hline & All Sizes & \multicolumn{2}{|c|}{ 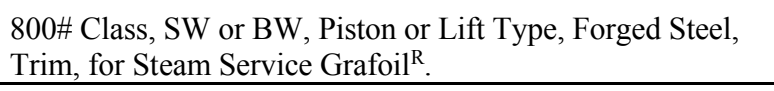 } & ASTM A105 ANSI B16.34 \\
\hline & $\begin{array}{l}\text { Same as } \\
\text { above } \\
\square\end{array}$ & \multicolumn{2}{|c|}{$\begin{array}{l}\text { 150\# Class, Cast Steel, BW, BB, Standard Trim, Swing } \\
\text { Type, Grafoil }{ }^{\mathrm{R}} \text { Gaskets. }\end{array}$} & ASTM A216 ANSI B16.34 \\
\hline $\begin{array}{l}\text { Check } \\
\text { Valves }\end{array}$ & 3" \& Larger & \multicolumn{2}{|c|}{$\begin{array}{l}\text { 150\# Class, Flanged, Cast Steel, Swing Type, Bolted Cap, } \\
\text { Swing Type, Grafoil }^{\mathrm{R}} \text { Gasket. }\end{array}$} & Same as above \\
\hline Flanges & All Sizes & \multicolumn{2}{|c|}{$\begin{array}{l}\text { 150\# ANSI B16.5, RF, Forged CS Weld Neck, All Sizes } \\
\text { Slip-on or SW. }\end{array}$} & $\begin{array}{l}\text { ASTM A181 ASTM A105 ANSI } \\
\text { B16.5 }\end{array}$ \\
\hline $\begin{array}{l}\text { Orifice } \\
\text { Flanges }\end{array}$ & $2 "-8 "$ & \multicolumn{2}{|c|}{$\begin{array}{l}\text { Forged Carbon Steel, 300\# Raised Face, Bored to match } \\
\text { pipe. }\end{array}$} & ASTM A105 ANSI B16.36 \\
\hline & $1 / 2 "-24 "$ & \multicolumn{2}{|c|}{ Grafoil $^{\mathrm{R}}$ Gaskets, 1/16" Thick. } & ANSI B16.21 \\
\hline Gaskets & & \multicolumn{2}{|c|}{ Note: Use 300\# Ring Type with Orifice Flanges. } & \\
\hline \multicolumn{2}{|c|}{ Bolting } & \multicolumn{2}{|c|}{$\begin{array}{l}\text { Chrome-moly, Stud Bolt Threaded Full Length Two } \\
\text { Semi-Finished Heavy Hex Nuts Each. }\end{array}$} & $\begin{array}{l}\text { ASTM A193 Grade B7 ASTM } \\
\text { A194 Grade 2H }\end{array}$ \\
\hline & $\underline{\text { Run Size }}$ & Branch Size & $\underline{\text { Use }}$ & \\
\hline $\begin{array}{l}\text { Branch } \\
\text { Connection }\end{array}$ & $\begin{array}{l}2 " \& \\
\text { Smaller }\end{array}$ & $\begin{array}{l}\text { Full Size or Reducing } \\
\text { Full }\end{array}$ & $\begin{array}{l}\text { Tee } \\
\text { Tee }\end{array}$ & \\
\hline
\end{tabular}


PIPING MATERIAL STANDARDS

Carbon Steel-Weld Fittings, 150 psi Service Rating, Line Class NC

\begin{tabular}{|c|c|c|c|c|}
\hline TYPE & PIPE SIZE & & ESCRIPTION & CODE \\
\hline $\mathrm{s}$ & $\begin{array}{l}\text { Larger than } \\
2^{2}\end{array}$ & $\begin{array}{l}\text { 2" and Smaller } \\
\text { Larger than 2" }\end{array}$ & $\begin{array}{l}\text { Saddle } \\
\text { Stub-in }\end{array}$ & \\
\hline
\end{tabular}


PIPING MATERIAL STANDARDS

\section{Chlorinated Polyvinyl Chloride (CPVC) CPVC (Temperature Limit 180EF) Line Class ND}

\section{$\underline{\text { Design Notes }}$}

1. When assembling nonmetallic flanges, flat washers shall be used under all bolt heads \& nuts.

2. When plastic pipe is used for potable water in buildings, CPVC shall be used on all hot and cold potable water piping.

3. Bronze or iron body valves may be used at designers options.

4. Use CPVC primer and cement for solvent welding of SW fittings.

5. See manufacturers recommendations for cleaning and joining CPVC pipe and fittings.

6. This line class shall not be used in contact with or within 10' of 300 series austenitic stainless steel line classes to avoid chloride contamination.

\begin{tabular}{|c|c|c|c|}
\hline TYPE & PIPE SIZE & MATERIAL DESCRIPTION & CODE \\
\hline Pipe & $1 / 2 "-6 "$ & $\begin{array}{l}\text { Hi Temperature CPVC, Plain Ends for SW (Preferred) or } \\
\text { SCRD. Schedule } 80 .\end{array}$ & ASTM F441 \\
\hline & $1 / 2^{\prime \prime}-3 "$ & CPVC Fittings, SW (Preferred) or SCRD, Schedule 80. & ASTM F437 or ASTM F439 \\
\hline Fittings & $1 "-6 "$ & $\begin{array}{l}\text { CPVC Fittings, 150\# ANSI B16.5 Flanged, Schedule } 80 \\
\text { SW (Preferred) or SCRD. }\end{array}$ & $\begin{array}{l}\text { Same as above } \\
\text { ANSI B } 16.5\end{array}$ \\
\hline Ball Valves & $1 / 2^{\prime \prime}-4^{\prime \prime}$ & CPVC, SCRD, SW or Flanged. True Union Type. & See Note 3 \\
\hline Flanges & $1 / 2 "-6 "$ & CPVC, SW (Preferred) or SCRD 150\# ANSI B16.5. & $\begin{array}{l}\text { ASTM F437 or ASTM F439 See } \\
\text { Note } 1 \text { ANSI B16.5 }\end{array}$ \\
\hline \multicolumn{2}{|c|}{ Gaskets } & TFE, Full Face - Non Radiation Use Only & \\
\hline \multicolumn{2}{|c|}{ Bolting } & Carbon Steel Bolts \& Nuts & See Note 1 ASTM A307 Grade B \\
\hline
\end{tabular}




\section{PIPING MATERIAL STANDARDS}

Vitrified Clay, Extra Strength Uniform Building Code, Line Class NE

\begin{tabular}{|c|l|l|l|}
\hline TYPE & PIPE SIZE & \multicolumn{1}{|c|}{ MATERIAL DESCRIPTION } & \multicolumn{1}{c|}{ CODE } \\
\hline Pipe & $2 "-4 "$ & Vitrified Clay Pipe, Extra Strength & ASTM C700 \\
\hline Fittings & $2 "-4 "$ & $\begin{array}{l}\text { Vitrified Clay, Extra Strength Molded Rubber Compression } \\
\text { Joints }\end{array}$ & ASTM C700 ASTM C425 \\
\hline
\end{tabular}


PIPING MATERIAL STANDARDS

\section{Carbon Steel - Polypropylene Lined (PPL), 150 psi Service Rating, Line Class NF}

\section{Design Notes}

1. All spool assemblies involving lined piping shall be fabricated from PPL lined carbon steel pipe w/150\# ductile iron flanges and flanged ductile iron, or cast steel PPL lined fittings.

2. Straight sections of pipe available in standard spool lengths of 10 feet and 20 feet.

3. Manufacturer's suggested bolt torque shall be used in assembling flange joints.

4. No gaskets required between flanges with molded raised faces.

5. The following pressure limitations shall be followed for diaphragm valves at $100^{\circ} \mathrm{F}$.

$$
\begin{array}{llll}
\frac{\text { Size }}{1 / 2 "-1 "} & \frac{\text { Max. }}{\text { Pressure }} & \frac{\text { Size }}{200 \text { psig }} & \begin{array}{r}
\text { Max. } \\
\text { Pressure }
\end{array} \\
1-1 / 2 "-2 " & \frac{175 \text { psig }}{150 \text { psig }} & \text { 6" only } & 125 \text { psig }
\end{array}
$$

\begin{tabular}{|c|c|c|c|}
\hline TYPE & PIPE SIZE & MATERIAL DESCRIPTION & CODE \\
\hline Pipe & $1 "-6 "$ & $\begin{array}{l}\text { Carbon Steel, Standard Weight, lined with Polypropylene, } \\
\text { 150\# ANSI B16.5 Screwed Ductile Iron Flange Ends. }\end{array}$ & $\begin{array}{l}\text { ASTM A } 53 \text { Grade B ASTM A } 395 \\
\text { ANSI B16.5 }\end{array}$ \\
\hline Fittings & $1 "-6 "$ & $\begin{array}{l}\text { Ductile Iron Flanged Fittings, 150\# ANSI B16.5 Lined } \\
\text { with Polypropylene, Molded Raised Face. }\end{array}$ & ASTM A395 ANSI B16.5 \\
\hline \multicolumn{2}{|c|}{ Check Valves } & 150\# Class, RF, Cast Steel, Polypropylene Lined. & $\begin{array}{l}\text { ASTM A216 Grade WCB } \\
\text { Dimensions per ANSI B16.34 }\end{array}$ \\
\hline & $1 "$ - 4" & $\begin{array}{l}\text { 150\# Class, RF, Cast Steel, Polypropylene Lined Furnished } \\
\text { with Wrench. }\end{array}$ & $\begin{array}{l}\text { ASTM A } 216 \text { Grade WCB } \\
\text { Dimensions per ANSI B16.34 }\end{array}$ \\
\hline $\begin{array}{l}\text { Plug } \\
\text { Valves } \\
\end{array}$ & 6" only & \multicolumn{2}{|l|}{$\begin{array}{l}\text { Same as above except furnished with enclosed worm gear } \\
\text { operator. }\end{array}$} \\
\hline $\begin{array}{l}\text { Diaphragm } \\
\text { Valves }\end{array}$ & $1 / 2 "-6 "$ & $\begin{array}{l}\text { 150\# Class, RF, Polypropylene Lined Cast Carbon Steel, } \\
\text { Hand Wheel Operated.1/2" - 6" }\end{array}$ & $\begin{array}{l}\text { ASTM A } 216 \text { Grade WCB } \\
\text { Dimensions per ANSI B16.34 }\end{array}$ \\
\hline & $1 "-6 "$ & $\begin{array}{l}\text { 150\# ANSI B16.5, Ductile Iron, Screwed, Chamfered } \\
\text { Acceptable: DOW } \# 520^{\mathrm{R}} \text { or equal. To be used with } \\
\text { Polypropylene Lining. }\end{array}$ & ASTM A395 ANSI B16.5 \\
\hline $\begin{array}{l}\text { Flanges } \\
\text { (Loose) }\end{array}$ & $1 "-6 "$ & $\begin{array}{l}\text { 150\# ANSI B16.5, Ductile Iron Blind Flanges, (use with } \\
\text { Polypropylene Full Face Blind Spacer) Acceptable: DOW } \\
\# 522^{\mathrm{R}} \text { or equal. }\end{array}$ & ASTM A395 ANSI B16.5 \\
\hline \multicolumn{2}{|c|}{ Orifice Flanges (Loose) } & $\begin{array}{l}\text { 300\# ANSI B16.5, Ductile Iron, Screwed, Chamfered with } \\
\text { 1/2" SCRD Taps. To be used with Polypropylene Lining. }\end{array}$ & ASTM A395 ANSI B16.36 \\
\hline \multicolumn{2}{|c|}{ Spacers } & $\begin{array}{l}\text { Use Standard Full-Face or Reducing Full Face PPL Spacer } \\
\text { when mating PPL lined piping items with all other types of } \\
\text { flanged piping. }\end{array}$ & \\
\hline \multicolumn{2}{|c|}{ Bolting } & Hex Head Machine Bolt with Hex Nut. & ASTM A307 Grade B \\
\hline Branches & $1 "-6 "$ & Use Full or Reducing PPL Lined Flanged Fittings. & \\
\hline
\end{tabular}


PIPING MATERIAL STANDARDS

\section{Polyethylene (Very High Molecular Weight High Density Polyethylene) Line Class NH}

\section{Design Notes}

1. VHMWHD Polyethylene used for service waste shall be used only in out-of-cell areas and only where compatible with the service waste material.

2. See ASME B31.3 Chapter VII "Nonmetallic Piping and Piping Lined with Non-metals" and ICPP Materials Development Organization for further direction on specifying material for the intended application and obtain prior written approval of ICPP Design Engineering Organization.

\begin{tabular}{|c|l|l|l|}
\hline TYPE & PIPE SIZE & \multicolumn{1}{|c|}{ MATERIAL DESCRIPTION } & \multicolumn{1}{c|}{ CODE } \\
\hline Pipe & $3 / 4 "$ - 14" & $\begin{array}{l}\text { VHMWHD Polyethylene as manufactured by Phillips } \\
\text { Driscopipe No. 8600 } \\
160 \text { psi or as required. }\end{array}$ & None \\
\hline Fittings & All Sizes & $\begin{array}{l}\text { VHMWHD Polyethylene as manufactured by Phillips } \\
\text { Driscopipe No. 8600 } 0^{\mathrm{R}} \text { or equal. Pressure Rating to match } \\
\text { pipe system rating. Ends suitable for butt-fusion. }\end{array}$ & None \\
\hline Special Fittings & $\begin{array}{l}\text { Molded Stub Ends of VHMWHD Polyethylene as } \\
\text { manufactured by Phillips - Driscopipe No. 8600 } \\
\text { shall or equal used in conjunction with Slip-on Flanges for } \\
\text { interface between VHMWHDPE Pipe and Metallic Pipe. } \\
\text { Pressure Rating to match pipe system rating. }\end{array}$ & None \\
\hline Flanges & $\begin{array}{l}\text { Slip-On (Flat Metal Plate) 304 SST 150 psi Driscopipe }{ }^{\mathrm{R}} \text { or } \\
\text { equal. }\end{array}$ & ANSI B16.21 \\
\hline Gaskets & $\begin{array}{l}\text { Gylon }{ }^{\mathrm{R}} \text { Ring Type, 1/16" Thick 150 psi } \\
\text { Bolts }\end{array}$ & $\begin{array}{l}\text { Use 304 SST Bolts ASTM A193 Gr B8 and Galling } \\
\text { Resistant Nitronic 60 } \text { Nuts ASTM A194 Gr 8S (UNS } \\
\text { S21800) }\end{array}$ & $\begin{array}{l}\text { ASTM A193 Gr B8 ASTM A194 } \\
\text { Gr 8S UNS S21800 }\end{array}$ \\
\hline
\end{tabular}




\section{PIPING MATERIAL STANDARDS}

Concrete, Line Class NI

\begin{tabular}{|c|l|l|l|}
\hline TYPE & PIPE SIZE & \multicolumn{1}{|c|}{ MATERIAL DESCRIPTION } & \multicolumn{1}{c|}{ CODE } \\
\hline Pipe & 4" \& Larger & Concrete Sewer, Storm Drain, and Culvert Pipe & ASTM C14 \\
\hline Joints & 4" \& Larger & $\begin{array}{l}\text { Joints for ASTM C443 Sewer, Storm drain and culvert pipe } \\
\text { shall be rubber gasketed belled ends. }\end{array}$ & ASTM C443 \\
\hline
\end{tabular}


PIPING MATERIAL STANDARDS

\section{Galvanized Carbon Steel, 125 - 150 psi Service Rating, Line Class NJ}

\section{Design Notes}

1. Fittings and equipment in this line class for firewater lines or raw water lines supplying fire water mains shall be specified per NFPA-13 and Factory Mutual Data Sheet 2-8N for above ground piping and NFPA 24 and Factory Mutual Data Sheet 3-10 for underground piping in lieu of the fittings and equipment specified herein.

2. For ASME B31.3 Base Category fluids see ASME B31.3 Table 314.2.1 for minimum pipe schedule requirements instead of those listed below. Match fitting class to pipe rating.

\begin{tabular}{|c|c|c|c|}
\hline TYPE & PIPE SIZE & MATERIAL DESCRIPTION & CODE \\
\hline Pipe & $1 / 2 "$ - 4" & $\begin{array}{l}\text { Carbon Steel, Galvanized, Sch 40, Threaded and Coupled } \\
\text { Ends. }\end{array}$ & ASTM A53 \\
\hline Fittings & All sizes & 150\# Class, Galvanized Malleable Iron Screwed. & ASTM A197 \\
\hline \multirow[b]{2}{*}{$\begin{array}{l}\text { Gate } \\
\text { Valves }\end{array}$} & All Sizes & 125\# Class Bronze, SCRD, Double Wedge, Rising Stem. & ASTM B62 \\
\hline & $\begin{array}{l}\text { Same as } \\
\text { above } \\
\square\end{array}$ & $\begin{array}{l}\text { 125\# Class, Iron Body, Flat-Faced Flanged, Double Wedge, } \\
\text { Non-Rising Stem, Bronze Trim. }\end{array}$ & ASTM A126 Class B \\
\hline \multirow[b]{4}{*}{$\begin{array}{l}\text { Globe } \\
\text { Valves }\end{array}$} & All Sizes & $\begin{array}{l}\text { 125\# Class, Bronze, SCRD, Rising Stem, Bronze Disc and } \\
\text { Seats, GrafoiL }{ }^{\mathrm{R}} \text { Packing and Gasket. }\end{array}$ & ASTM B62 \\
\hline & $\begin{array}{l}\text { Same as } \\
\text { above } \\
\square\end{array}$ & $\begin{array}{l}\text { 150\# Class, Bronze, SCRD, Rising Stem, Renewable } \\
\text { Composition Disc, Bronze Seat, Grafoil }{ }^{\mathrm{R}} \text { Packing and } \\
\text { Gasket. }\end{array}$ & ASTM B62 \\
\hline & $\begin{array}{l}\text { Same as } \\
\text { above } \\
\square\end{array}$ & $\begin{array}{l}\text { 125\# Class, Iron Body, Flat-Faced Flanged, OS\&Y Bolted } \\
\text { Bonnet, Rising Stem, Solid Bronze Disc, Trim and Seats. }\end{array}$ & ASTM A126 Class B \\
\hline & $\begin{array}{l}\text { Same as } \\
\text { above } \\
\square\end{array}$ & $\begin{array}{l}\text { 125\# Class, Iron Body, Raised Face Flanged, OS\&Y Bolted } \\
\text { Bonnet, Rising Stem, Composition Disc \& Seats. }\end{array}$ & ASTM B62 \\
\hline \multirow[b]{3}{*}{$\begin{array}{l}\text { Check } \\
\text { Valves }\end{array}$} & All Sizes & $\begin{array}{l}\text { 125\# Class, OS\&Y, Bronze, Screwed, Full Way Bronze } \\
\text { Discs and Seats. }\end{array}$ & ASTM B62 \\
\hline & $\begin{array}{l}\text { Same as } \\
\text { above } \\
\square\end{array}$ & $\begin{array}{l}\text { 150\# Class, Bronze, Screwed, Renewable Composition } \\
\text { Disc, Seats. }\end{array}$ & ASTM B62 \\
\hline & All Sizes & $\begin{array}{l}125 \# \text { Class, Iron Body, Bronze Mtd., Flanged, Horizontal } \\
\text { Swing. }\end{array}$ & ASTM B62 \\
\hline \multirow[t]{2}{*}{$\begin{array}{l}\text { Service } \\
\text { Stops }\end{array}$} & $\begin{array}{l}\text { Same as } \\
\text { above } \\
\square\end{array}$ & 125\# ANSI, Bronze, SCRD, Square or Flat Head. & ASTM B62 \\
\hline & & 125\# ANSI, Iron Body, SCRD, Square Head. & ASTM A126 Class B \\
\hline \multirow[b]{2}{*}{ Ball Valves } & $1 / 4^{\prime \prime}-2^{\prime \prime}$ & $\begin{array}{l}\text { 300\# Class WOG, SCRD, Bronze Body TFE Seats and } \\
\text { Seals, Lever Operated. }\end{array}$ & ASTM B62 \\
\hline & $3 "-4 "$ & $\begin{array}{l}\text { 150\# Class, Raised-Face Flange, Ductile Iron Body, TFE } \\
\text { Seat, Top Entry, Lever Operated. }\end{array}$ & ASTM A395 \\
\hline $\begin{array}{l}\text { Plug } \\
\text { Valves }\end{array}$ & $1 / 2^{\prime \prime}-2^{\prime \prime}$ & $\begin{array}{l}\text { 200\# Class, SCRD, Cast Iron Body and Plug, TFE Sleeve } \\
\text { and Diaphragm, Lever operated. }\end{array}$ & ASTM A126 Class B \\
\hline
\end{tabular}


PIPING MATERIAL STANDARDS

Galvanized Carbon Steel, 125 - 150 psi Service Rating, Line Class NJ

\begin{tabular}{|c|l|l|l|}
\hline TYPE & PIPE SIZE & \multicolumn{1}{|c|}{ MATERIAL DESCRIPTION } & \multicolumn{1}{c|}{ CODE } \\
\hline Flanges & $2^{\prime \prime}-4^{\prime \prime}$ & Galvanized Malleable Iron 150\# FF, Screwed Flange. & ASTM A105 \\
\hline $\begin{array}{c}\text { Orifice } \\
\text { Flanges }\end{array}$ & All Sizes & $\begin{array}{l}\text { Forged Carbon Steel, 300\# RF, Weld Neck Type, with 1/2" } \\
\text { SCRD Tops, with bore to match Pipe. }\end{array}$ & ASTM A105 \\
\hline \multirow{2}{*}{ Gaskets } & $1 / 4^{\prime \prime}-6^{\prime \prime}$ & Grafoil ${ }^{\mathrm{R}}$ flange gasket, flat ring, 1/16" Thick. & ANSI B16.21 \\
\hline \multicolumn{2}{|c|}{ Bolting } & Hex head machine bolt with hex nut. & ASTM A307 Grade B \\
\hline & $1 / 4^{\prime \prime}-2^{\prime \prime}$ & For full size or reducing branch, use full or reducing tee. & \\
\cline { 2 - 5 } Branches & $3^{\prime \prime}-6^{\prime \prime}$ & For full size branch, use full size tee. & \\
\hline
\end{tabular}


PIPING MATERIAL STANDARDS

\section{Copper Water Tubing Type L or K, Line Class NK}

\section{Design Notes}

1. Note restrictions on use of tubing in Section 5.6.2 of this A-E Standard.

\begin{tabular}{|c|l|l|l|}
\hline TYPE & PIPE SIZE & \multicolumn{1}{|c|}{ MATERIAL DESCRIPTION } & CODE \\
\hline Tubing & $1 / 4 "-2 "$ & $\begin{array}{l}\text { Seamless Copper Water Tubing, Hard Drawn or Soft } \\
\text { Drawn, Type L or K Straight Length or Coil. }\end{array}$ & ASTM B88 \\
\hline Fittings & $1 / 4 "$-2" & Wrought Copper or Bronze Solder - Type Pressure Fittings. & ANSI B16.22 \\
\hline \multicolumn{2}{|c|}{ Soldering Material } & Silver Brazing Wire. & \\
\hline $\begin{array}{c}\text { Gate } \\
\text { Valves }\end{array}$ & $1 / 4 "-2 "$ & $\begin{array}{l}\text { 125\# Class, Bronze, Double Wedge Rising Stem } \\
\text { Screwed-In Bonnet, Solder Joint Tubing End or SCRD. }\end{array}$ & ASTM B62 \\
\hline $\begin{array}{c}\text { Globe } \\
\text { Valves }\end{array}$ & $1 / 4 "-2 "$ & $\begin{array}{l}\text { 150\# Class, Bronze, Renewable Composition Disc, Rising } \\
\text { Stem, Union Bonnet, Solder Joint Tubing End or SCRD. }\end{array}$ & ASTM B62 \\
\hline $\begin{array}{c}\text { Check } \\
\text { Valves }\end{array}$ & $1 / 4 "-2 "$ & $\begin{array}{l}\text { 125\# Class, Bronze, Screwed-In Cap, Bronze Disc, Solder } \\
\text { Joint Tubing End or SCRD. }\end{array}$ & ASTM B62 \\
\hline
\end{tabular}




\section{PIPING MATERIAL STANDARDS}

\section{Polypropylene, Line Class NL}

\section{Design Notes}

1. Polypropylene piping systems shall be fabricated in strict accordance with the manufacturer's recommendations.

2. Installation practices, including flange bolt torque, support spacing, and expansion considerations shall be in compliance with the manufacturer's recommendations.

3. Joints in underground and trench piping shall be made by fusion. Break-out joints in above ground piping shall be made w/flanges.

4. Installed piping shall be pressure tested before putting it into service in accordance with the manufacturer's recommendations.

5. TFE not suitable for radiation area use. In radiation areas ring material shall be selected for particular radiation fields and chemical compatibility.

6. When assembling nonmetallic flanges, flat washers shall be used under all bolt heads and nuts.

7. Original installation in FDP was ASTM 2146 (replaced by D4101) Type 1-29209 with thickness and tolerance per ASTM D-1785. However, these are not specific PP pipe standards. See ASME B31.3 Chapter VII "Nonmetallic Piping and Piping Lined with Nonmetals" and ICPP Materials Development Organization for further application and obtain prior written approval of ICPP Design Engineering Organization.

\begin{tabular}{|c|c|c|c|}
\hline TYPE & PIPE SIZE & MATERIAL DESCRIPTION & CODE \\
\hline Pipe & $1 / 2^{\prime \prime}-6^{\prime \prime}$ & Polypropylene pipe, Schedule 80 & Note 7 \\
\hline Fittings & All Sizes & Polypropylene Fittings, Socket Ends, Schedule 80 & Note 7 \\
\hline Ball Valves & 1/2" - 6" & $\begin{array}{l}\text { Ball Valves, } 150 \text { psi, CWP, True Union, Polypropylene } \\
\text { Body with TFE Seats, and TEE O-Ring Seal, Socket Ends } \\
\text { with Socket Diameter compatible with Schedule } 80 \\
\text { Polypropylene Pipe for Fusion Joining. Handle Operated. }\end{array}$ & See Note 5 \\
\hline \multicolumn{2}{|c|}{ Flanges } & Polypropylene Flanges, Socket Ends, 150\# FF ANSI B16.5. & $\begin{array}{l}\text { ASTM D2146 Type 1-29209 ANSI } \\
\text { B16.5 See Note } 6\end{array}$ \\
\hline \multicolumn{2}{|c|}{ Gaskets } & $\begin{array}{l}\text { Hypalon }^{\mathrm{R}} \text { Gaskets for Polypropylene Flanges, Viton }{ }^{\mathrm{R}} \\
\text { Gaskets in Radiation Area, 150\# FF ANSI B16.5 Drilling } \\
\text { Full Face w/Bolt Holes, 1/8" Thick. }\end{array}$ & ANSI B16.5 \\
\hline \multicolumn{2}{|c|}{ Bolts } & Hex Head machine Bolts w/Hex Head Nuts. & ASTM A307 Grade B See Note 6 \\
\hline
\end{tabular}


PIPING MATERIAL STANDARDS

Copper Tubing (Refrigeration), Line Class NM

\begin{tabular}{|c|c|c|c|}
\hline TYPE & PIPE SIZE & MATERIAL DESCRIPTION & CODE \\
\hline Tube & $\begin{array}{l}2 / 8^{\prime \prime}-6-1 / 8^{\prime \prime} \\
\text { O. D. }\end{array}$ & $\begin{array}{l}\text { Seamless Refrigeration Tubing Hard Drawn, Straight } \\
\text { Length. }\end{array}$ & ASTM B280 UNS \#Cl2200 \\
\hline Fittings & $\begin{array}{l}3 / 8^{\prime \prime}-6-1 / 8^{\prime \prime} \\
\text { O. D }\end{array}$ & Wrought Copper Solder - Type Pressure Fittings. & ANSI B16.22 \\
\hline \multicolumn{2}{|c|}{ Soldering Material } & Silver Brazing Wire. & \\
\hline \multicolumn{2}{|c|}{ Gate Valves } & $\begin{array}{l}\text { 125\# Class, Bronze, Double Wedge, Rising Stem, Screwed } \\
\text { Bonnet, Solder Joint Tubing End or SCRD. }\end{array}$ & ASTM B62 \\
\hline \multicolumn{2}{|c|}{ Globe Valves } & $\begin{array}{l}\text { 150\# Class, Bronze, Renewable Composition Disc, Rising } \\
\text { Stem, Union Bonnet, Solder Joint Tubing End or SCRD. }\end{array}$ & ASTM B62 \\
\hline \multicolumn{2}{|c|}{ Check Valves } & $\begin{array}{l}\text { 125\# Class, Bronze, Screwed-In Cap, Bronze Disc, Solder } \\
\text { Joint Tubing End or SCRD. }\end{array}$ & ASTM B62 \\
\hline
\end{tabular}




\section{PIPING MATERIAL STANDARDS}

\section{Carbon Steel, 150 psi Service Rating, Line Class NN}

\section{Design Notes}

1. On all buried lines except firewater lines or raw water lines supplying fire mains, consideration should be given to welding all joints. See body of this standard for further direction.

2. Fittings and equipment in this line class for firewater lines or raw water lines supplying firewater mains shall be specified per NFPA-13 and Factory Mutual Data Sheet 2-8N for above ground piping and NFPA 24 and Factory Mutual Data Sheet 3-10 for underground piping in lieu of the fittings and equipment specified herein.

3. For high temperature service $\left(250^{\circ}\right)$.

\begin{tabular}{|c|c|c|c|}
\hline TYPE & PIPE SIZE & MATERIAL DESCRIPTION & CODE \\
\hline \multirow[b]{2}{*}{ Pipe } & $\begin{array}{l}2 " \text { and } \\
\text { Smaller }\end{array}$ & Seamless Black Carbon Steel, SCH 40 & ASTM A106 (3) Grade B \\
\hline & All Sizes & Carbon Steel Schedule 40 & ASTM A53 \\
\hline \multirow[b]{2}{*}{ Fittings } & $\begin{array}{l}2 " \& \\
\text { Smaller }\end{array}$ & $\begin{array}{l}\text { 150\# Class, Malleable Iron, SCRD or } 3000 \# \text { Class, Forged } \\
\text { Carbon Steel, SW or SCRD. }\end{array}$ & $\begin{array}{l}\text { ASTM A197 or ASTM A105 See } \\
\text { Note } 3\end{array}$ \\
\hline & All Sizes & Seamless Wrought Carbon Steel BWE, Sch 40. & ASTM A234 Grade WPB \\
\hline \multirow[b]{4}{*}{$\begin{array}{l}\text { Gate } \\
\text { Valves }\end{array}$} & $\begin{array}{l}2^{\prime \prime} \& \\
\text { Smaller }\end{array}$ & $\begin{array}{l}\text { 800\# Class, Forged Steel, SW or BW, OS\&Y, BB, Trim for } \\
\text { Steam Service, Grafoil }{ }^{\mathrm{R}} \text { Packing and Gaskets. }\end{array}$ & ASTM A 105 Note 3 \\
\hline & $\begin{array}{l}\text { Larger than } \\
2^{\prime \prime}\end{array}$ & $\begin{array}{l}\text { 150\# Class, Cast Steel, Flanged, OS\&Y, BB, STD Trim, } \\
\text { Grafoil }^{\mathrm{R}} \text { Packing and Gaskets. }\end{array}$ & ASTM A216 Class B \\
\hline & All Sizes & $\begin{array}{l}\text { 150\# Class, Bronze, Cast Iron or Malleable Iron, SCRD, } \\
\text { OS\&Y, BB Standard Trim, Grafoil }{ }^{\mathrm{R}} \text { Packing and Gaskets. }\end{array}$ & $\begin{array}{l}\text { ASTM A126 Class B ASTM B } 61 \\
\text { ASTM B } 62\end{array}$ \\
\hline & $\begin{array}{l}\text { Same as } \\
\text { above } \\
\square\end{array}$ & $\begin{array}{l}\text { 150\# Class, Cast Steel, BW, OS\&Y, BB Standard Trim, } \\
\text { Grafoil }^{\mathrm{R}} \text { Packing and Gaskets. }\end{array}$ & ASTM A216 \\
\hline \multirow[b]{4}{*}{$\begin{array}{l}\text { Globe } \\
\text { Valves }\end{array}$} & $\begin{array}{l}2^{\prime \prime} \& \\
\text { Smaller }\end{array}$ & $\begin{array}{l}\text { 800\# Class, Forged Steel, SW or BW, OS\&Y, BB, Trim for } \\
\text { Steam Service, Renewable Disc \& Seat Grafoil }{ }^{\mathrm{R}} \text { Packing } \\
\text { and Seat. }\end{array}$ & ASTM A105 Note 3 \\
\hline & All Sizes & $\begin{array}{l}\text { 150\# Class, Bronze, Cast Iron or Malleable Iron, SCRD, } \\
\text { OS\&Y, BB, Standard Trim, Grafoil }{ }^{\mathrm{R}} \text { Packing and Gaskets. }\end{array}$ & $\begin{array}{l}\text { ASTM A } 126 \text { Class B ASTM B } 61 \\
\text { ASTM B } 62\end{array}$ \\
\hline & $\begin{array}{l}\text { Same as } \\
\text { above } \\
\square\end{array}$ & $\begin{array}{l}\text { 150\# Class, Cast Steel, BW, OS\&Y, BB, Standard Trim, } \\
\text { Grafoil }^{\mathrm{R}} \text { Packing and Gaskets. }\end{array}$ & ASTM A216 \\
\hline & $\begin{array}{l}\text { Larger than } \\
2^{\prime \prime}\end{array}$ & $\begin{array}{l}\text { 150\# Class, Cast Steel, Flanged, OS\&Y, BB, Standard } \\
\text { Trim, Grafoil }{ }^{\mathrm{R}} \text { Packing and Gaskets. }\end{array}$ & ASTM A216 \\
\hline \multirow[b]{3}{*}{$\begin{array}{l}\text { Check } \\
\text { Valves }\end{array}$} & $\begin{array}{l}2^{\prime \prime} \& \\
\text { Smaller }\end{array}$ & $\begin{array}{l}\text { 800\# Class, SW or BW, Piston or Lift Type Forged Steel, } \\
\text { Trim for Steam Service, Grafoil }{ }^{\mathrm{R}} \text { Gasket. }\end{array}$ & ASTM A105 Note 3 \\
\hline & 3" \& Larger & $\begin{array}{l}\text { 150\# Class, Flanged, Cast Steel, Swing Type, Bolted Cap, } \\
\text { Swing Type, Grafoil }{ }^{\mathrm{R}} \text { Gasket. }\end{array}$ & ASTM A216 \\
\hline & All Sizes & $\begin{array}{l}\text { 150\# Class, Bronze, Cast Iron or Malleable Iron SCRD, BB, } \\
\text { Standard Trim, Grafoil }{ }^{\mathrm{R}} \text { Packing and Gaskets. }\end{array}$ & $\begin{array}{l}\text { ASTM A126 Class B ASTM B61 } \\
\text { ASTM B62 }\end{array}$ \\
\hline
\end{tabular}


PIPING MATERIAL STANDARDS

Carbon Steel, 150 psi Service Rating, Line Class NN

\begin{tabular}{|c|c|c|c|}
\hline TYPE & PIPE SIZE & MATERIAL DESCRIPTION & CODE \\
\hline & $\begin{array}{l}\text { Same as } \\
\text { above } \\
\square\end{array}$ & $\begin{array}{l}\text { 150\# Class, Cast Steel, BW, BB, Standard Trim, Grafoil } \\
\text { Packing and Gaskets. }\end{array}$ & ASTM A216 \\
\hline Ball Valves & $\begin{array}{l}2 " \& \\
\text { Smaller }\end{array}$ & $\begin{array}{l}\text { 300\# Class, Cast Steel Body, SCRD, or SW } 316 \text { SST Ball } \\
\text { \& Stem, CS Packing Retainer and Gland, TFE Ball Seat, } \\
\text { Grafoil }{ }^{\mathrm{R}} \text { Seal Ring \& Stem Packing. }\end{array}$ & ASTM A216 \\
\hline \multicolumn{2}{|c|}{ Butterfly Valves } & $\begin{array}{l}\text { 150\# Class, FF or Wafer-Type, Cast Iron, SST Shaft, Disc } \\
\text { and Seat to be determined for particular service. }\end{array}$ & ASTM A126 \\
\hline \multirow[b]{3}{*}{$\begin{array}{l}\text { Plug } \\
\text { Valves }\end{array}$} & $\begin{array}{l}2^{\prime \prime} \& \\
\text { Smaller }\end{array}$ & $\begin{array}{l}\text { 150\# Class, Malleable Iron, Screwed, TFE Sleeve and } \\
\text { Diaphragm. }\end{array}$ & ASTM A197 \\
\hline & 3" \& Larger & $\begin{array}{l}\text { 150\# Class, Cast Steel, Flanged, TFE Sleeve and } \\
\text { Diaphragm, Austenitic Stainless Steel Plug. }\end{array}$ & ASTM A216 \\
\hline & All Sizes & $\begin{array}{l}\text { 150\# Class, Cast Steel, BW, TFE Sleeve and Diaphragm, } \\
\text { Austenitic SST Plug. }\end{array}$ & ASTM A216 \\
\hline \multirow[b]{2}{*}{$\begin{array}{l}\text { Diaphragm } \\
\text { Valves }\end{array}$} & $1 / 2^{\prime \prime}-2^{\prime \prime}$ & $\begin{array}{l}\text { 125\# Class, WOG, Screwed Ends, Cast Iron, Ethylene } \\
\text { Propylene Diaphragm, Weir Type, Handwheel Operated } \\
\text { Indicating Stem. }\end{array}$ & ASTM A126 \\
\hline & $3 "-6 "$ & $\begin{array}{l}\text { 125\# Class, FF Flanged Ends, Cast Iron, Ethylene } \\
\text { Propylene Diaphragm, Weir Type, Handwheel Operated } \\
\text { Indicating Stem. }\end{array}$ & ASTM A126 \\
\hline \multirow[b]{2}{*}{ Flanges } & $\begin{array}{l}2 " \& \\
\text { Smaller }\end{array}$ & $\begin{array}{l}\text { 150\# ANSI B16.5, RF, Forged CS Weld Neck, Slip-on, } \\
\text { Threaded or SW. }\end{array}$ & $\begin{array}{l}\text { ASTM A105 ASTM A181 ANSI } \\
\text { B16.5 }\end{array}$ \\
\hline & 3" \& Larger & $\begin{array}{l}\text { 150\# ANSI B16.5, RF, Forged CS Weld Neck, Threaded or } \\
\text { Slip-on. }\end{array}$ & $\begin{array}{l}\text { ASTM A105 ASTM A181 ANSI } \\
\text { B16.5 }\end{array}$ \\
\hline $\begin{array}{l}\text { Orifice } \\
\text { Flanges }\end{array}$ & $2^{\prime \prime}-8 "$ & $\begin{array}{l}\text { Forged Carbon Steel, 300\# Raised Face, bored to match } \\
\text { pipe. }\end{array}$ & ASTM A105 ANSI B16.36 \\
\hline \multirow[t]{2}{*}{ Gaskets } & $1 / 2 "-24 "$ & 150\#, Grafoil ${ }^{\mathrm{R}}$ Gaskets, 1/16" Thick & ANSI B16.21 \\
\hline & & Note: Use $300 \#$ Configuration with Orifice Flanges. & \\
\hline \multicolumn{2}{|c|}{ Bolting } & $\begin{array}{l}\text { Chrome-moly, Stud bolt Threaded Full Length, Two Semi- } \\
\text { Finished Heavy Hex Nuts Each }\end{array}$ & $\begin{array}{l}\text { ASTM A193 Grade B7 ASTM } \\
\text { A194 Grade } 2 \mathrm{H}\end{array}$ \\
\hline
\end{tabular}




\section{PIPING MATERIAL STANDARDS}

\section{Polyvinyl Chloride - Drain, Waste and Vent (PVC-DWV) Uniform Plumbing Code PVC-DWV, Line Class NO}

\section{Design Notes}

1. Although referenced in the UPC, some of the fittings shown in ASTM D3311 are not acceptable under the UPC. See UPC for more details.

2. Use PVC cement for solvent welding of SW fittings. (ASTM D2564)

3. Use UPC for connections or transitions to bell and spigot pipe fittings.

4. See the UPC for installation standards for PVC-DWV.

\begin{tabular}{|c|l|l|l|}
\hline TYPE & PIPE SIZE & \multicolumn{1}{|c|}{ MATERIAL DESCRIPTION } & \multicolumn{1}{c|}{ CODE } \\
\hline Pipe & $2 "-4 "$ & $\begin{array}{l}\text { PVC-DWV, (SCH 40), Plain Ends for SW (Preferred) or } \\
\text { SCRD. }\end{array}$ & ASTM D2665 \\
\hline Fittings & $2 "$ - 4" & PVC-DWV SW (Preferred or SCRD. & $\begin{array}{l}\text { ASTM D2665 ASTM D3311 See } \\
\text { Note 1 See Note 3 }\end{array}$ \\
\hline
\end{tabular}




\section{PIPING MATERIAL STANDARDS}

\section{Copper Tubing, Line Class NP}

\section{Design Notes}

1. Note restrictions an use of tubing in Section 5.6.2 of the A-E Standard.

2. Where higher pressures are encountered, the designer has the option of selecting thicker-walled tubing.

\begin{tabular}{|c|c|c|c|}
\hline TYPE & PIPE SIZE & MATERIAL DESCRIPTION & CODE \\
\hline Tube & $1 / 8^{\prime \prime}-1 "$ & $\begin{array}{l}\text { Copper, } .035 " \text { Wall, Hard-drawn or soft-drawn Type K, } \\
\text { Straight Length or Coil. }\end{array}$ & ASTM B88 \\
\hline Fittings & $1 / 8^{\prime \prime}-1 "$ & Brass, Swagelok ${ }^{\mathrm{R}}$ Compression Type fittings & \\
\hline \multicolumn{2}{|c|}{ Valves } & $\begin{array}{l}\text { Brass, Whitey }{ }^{\mathrm{R}} \text { or Nupro }{ }^{\mathrm{R}} \text { with Swagelok }{ }^{\mathrm{R}} \text { Compression } \\
\text { Type Fittings, TFE Seats and Seals. }\end{array}$ & \\
\hline
\end{tabular}




\section{PIPING MATERIAL STANDARDS}

\section{Aluminum Tubing, Line Class NO}

\section{Design Notes}

1. Note restrictions an use of tubing in Section 5.6.2 of the A-E Standard.

2. Where higher pressures are encountered, the designer has the option of selecting thicker-walled tubing.

\begin{tabular}{|c|l|l|c|}
\hline TYPE & PIPE SIZE & \multicolumn{1}{|c|}{ MATERIAL DESCRIPTION } & CODE \\
\hline \multicolumn{2}{|c|}{ Tube } & Aluminum, Seamless, 0.035" Wall, Alloy 6061-T6. & Fed. WW-T-700/6 or ASTM B210 \\
\hline Fittings & $1 / 8^{\prime \prime}-1 "$ & Aluminum, Swagelok ${ }^{\mathrm{R}}$ Compression Type Fittings. $^{\prime \prime}$ & \\
\hline \multicolumn{2}{|c|}{ Valves } & $\begin{array}{l}\text { Aluminum, Whitey }{ }^{\mathrm{R}} \text { or Nupro }^{\mathrm{R}} \text { with Swagelok } \\
\text { Compression Type Fittings, TFE Seats and Seals. }\end{array}$ \\
\hline
\end{tabular}




\section{PIPING MATERIAL STANDARDS \\ Ductile Iron Water Pipe, Line Class NR}

\section{Design Notes}

1. Fittings and equipment in this line class for firewater lines or raw water lines supplying fire water mains shall be specified per NFPA-13 and Factory Mutual Data Sheet 2-8N for above ground piping and NFPA 24 and Factory Mutual Data Sheet 3-10 for underground piping in lieu of the fittings and equipment specified herein.

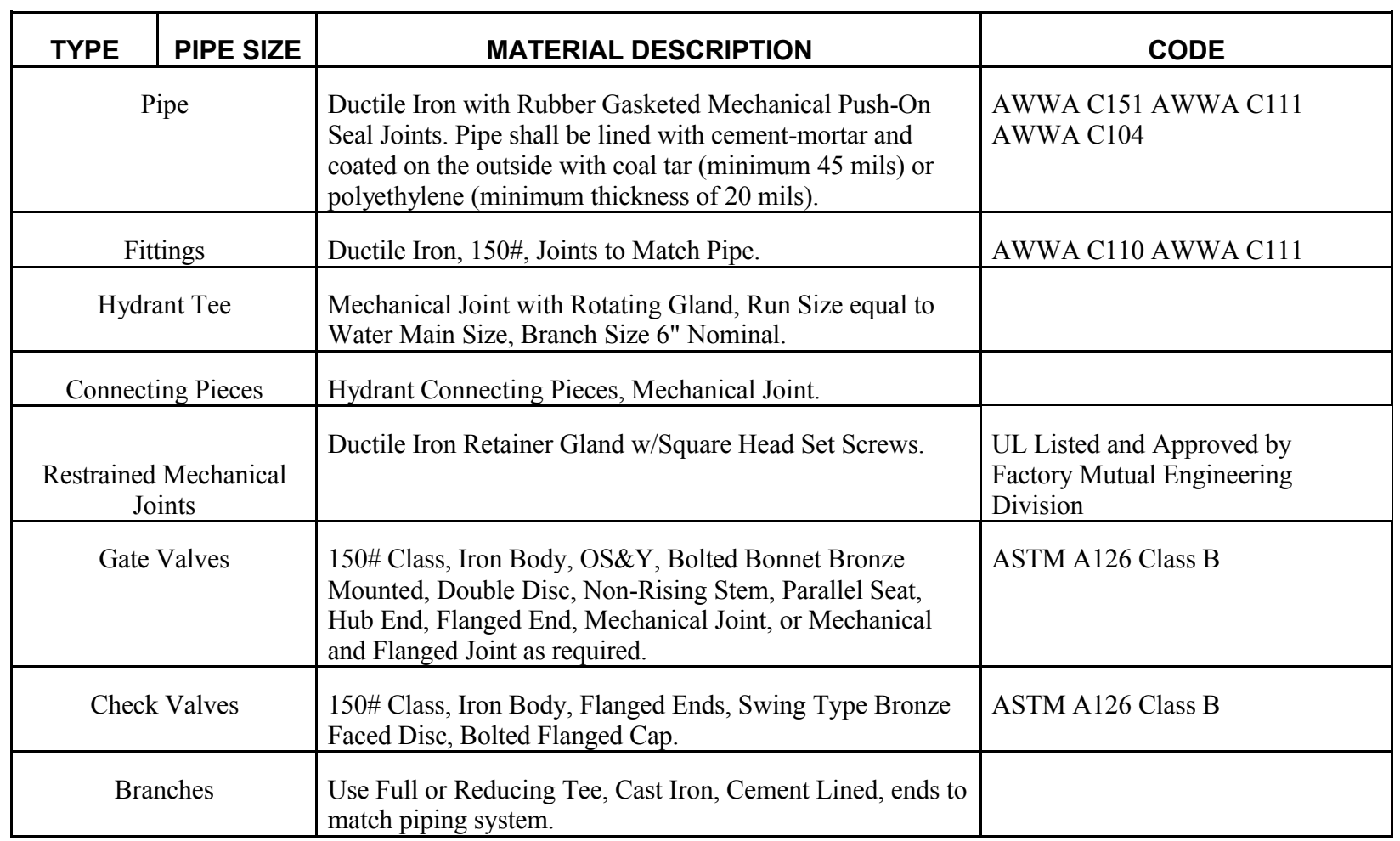


PIPING MATERIAL STANDARDS

\section{Fiberglass Reinforced Plastic (FRP), Line Class NS}

\section{Design Notes}

1. FRP type formulation and standard shall be determined for the particular service and associated corrosion and radiation requirements. Thermoplastic lined FRP shall be considered where corrosion is severe. See ASME B31.3 Chapter VII "Nonmetallic Piping and Piping Lined with Nonmetals" and ICPP Materials Development Organization for further direction on specifying material for the intended application and obtain prior written approval of ICPP Design Engineering Organization.

2. Valves for corrosion services shall be specified of materials suitable to withstand the particular corrosion service. 316 SST valves listed are for general use only.

3. TFE not for use in radiation areas.

4. When assembling nonmetallic flanges, flat washers shall be used under all bolt heads and nuts.

\begin{tabular}{|c|c|c|}
\hline PIPE SIZE & MATERIAL DESCRIPTION & CODE \\
\hline Pipe & 150\# Rated Fiberglass Reinforced Plastic. & Note 1 \\
\hline Fittings & $\begin{array}{l}\text { Bell \& Spigot or Flanged, Compatible w/Manufacturer's } \\
\text { System. Adapt to other systems w/Grooved, Threaded, or } \\
\text { Flange Adapters. }\end{array}$ & \\
\hline Gate Valves & $\begin{array}{l}\text { 150\# Class, RF, OS\&Y, Double Wedge, BB, } 316 \text { SST Body } \\
\& \text { Trim, Grafoil }{ }^{\mathrm{R}} \text { Packing and Gaskets. }\end{array}$ & $\begin{array}{l}\text { ASTM A351 Grade CF8M ASTM } \\
\text { A182 Grade F316 }\end{array}$ \\
\hline Globe Valves & $\begin{array}{l}\text { 150\# Class, RF, OS\&Y, BB, } 316 \text { SST Body and Trim, } \\
\text { Grafoil }^{\mathrm{R}} \text { Packing and Gasket }\end{array}$ & $\begin{array}{l}\text { ASTM A351 Grade CF8M ASTM } \\
\text { A182 Grade F316 }\end{array}$ \\
\hline Check Valves & $\begin{array}{l}\text { 150\# Class, RF, Wafer Check } 316 \text { SST Body, Plates and } \\
\text { Trim, Metal to Metal Seat, Inconel }{ }^{\mathrm{R}} \text { Spring. }\end{array}$ & $\begin{array}{l}\text { ASTM A351 Grade CF8M ASTM } \\
\text { A182 Grade F316 }\end{array}$ \\
\hline Ball Valves & $\begin{array}{l}\text { 150\# Class, RF, } 316 \text { SST Body, Ball \& Stem, TFE Seats \& } \\
\text { Body Seals, SST External Parts, Lever Operated. }\end{array}$ & $\begin{array}{l}\text { See Note } 3 \text { ASTM A351 Grade } \\
\text { CF8M ASTM A182 Grade F316 }\end{array}$ \\
\hline Butterfly Valves & $\begin{array}{l}\text { 150\# Class, Single Flange, Lug Type } 316 \text { SST Body \& } \\
\text { Disc, } 17-4 \text { pH Stem, Tefzel }{ }^{\mathrm{R}} \text { Seal Ring, EPR Back-up Ring, } \\
\text { Lever Lock Handle. }\end{array}$ & $\begin{array}{l}\text { See Note } 3 \text { ASTM A351 Grade } \\
\text { CF8M ASTM A182 Grade F316 }\end{array}$ \\
\hline Plug Valves & $\begin{array}{l}\text { 150\# Class, RF, } 316 \text { SST Body, Cover and Plug, UHMW } \\
\text { Polyethylene Sleeve and Diaphragm, Wrench Operated. }\end{array}$ & $\begin{array}{l}\text { See Note } 3 \text { ASTM A351 Grade } \\
\text { CF8M ASTM A182 Grade F316 }\end{array}$ \\
\hline Diaphragm Valves & $\begin{array}{l}\text { 150\# Class, RF, } 316 \text { SST Body, Bonnet and Stem, Viton }{ }^{\mathrm{R}} \\
\text { Rubber Diaphragm, Weir Type, Handwheel Operated. }\end{array}$ & $\begin{array}{l}\text { See Note } 3 \text { ASTM A } 351 \text { Grade } \\
\text { CF8M ASTM A182 Grade F316 }\end{array}$ \\
\hline Flanges & $\begin{array}{l}\text { 150\# ANSI B16.5, FRP Flanges, when mating to raised face } \\
\text { flanges or lug valves, use spacers to prevent damage to } \\
\text { fiberglass flanges. }\end{array}$ & See Note 4 ANSI B16.5 \\
\hline Gaskets & $\begin{array}{l}\text { Full Face, } 1 / 8^{\prime \prime} \text { Thick } 60 \text { Durometer. Torque as } \\
\text { recommended by manufacturer of flange. }\end{array}$ & \\
\hline Bolting & $\begin{array}{l}\text { Stud Bolts 18-8 SST ASTM A193, Grade B8, Class } 2 \text { with } \\
\text { Hex Nuts ASTM A194, Grade 8M }\end{array}$ & $\begin{array}{l}\text { ASTM A193 Grade B8 Class } 2 \\
\text { ASTM A194 Grade 8M See Note } 4\end{array}$ \\
\hline Line Reduction & Use Concentric Flanged or Bell \& Spigot Reducers. & \\
\hline Branches & $\begin{array}{l}\text { Full Size - Use Tee } \\
\text { Reducing - Use Saddle }\end{array}$ & \\
\hline
\end{tabular}




\section{PIPING MATERIAL STANDARDS \\ Fiberglass Reinforced Plastic (FRP), Line Class NS}

\section{Design Notes}

1. On the original NWCF specification SP-453504-50-3 cast iron was designated as class NE. All future installations throughout ICPP will be designated as class NT.

\begin{tabular}{|c|l|l|l|}
\hline TYPE & PIPE SIZE & \multicolumn{1}{|c|}{ MATERIAL DESCRIPTION } & CODE \\
\hline Pipe & $4 "-12 "$ & $\begin{array}{l}\text { Cast Iron Soil Type, Bell and Spigot, Extra Heavy, Five or } \\
\text { Ten Foot Lengths. (Nominal Sizes 4, 6, 8, 10 and 12.) }\end{array}$ & ASTM A74 \\
\hline Fittings & $4 "-12 "$ & Cast Iron Soil Type, Bell and Spigot, Extra Heavy. & ASTM A74 \\
\hline \multicolumn{2}{|c|}{ Clean Outs } & $\begin{array}{l}\text { Cast Iron Soil Type, with Cored Square Head Brass Plug, } \\
\text { Bell and Spigot Ends, Extra Heavy. }\end{array}$ & ASTM A74 \\
\hline \multicolumn{2}{|c|}{ Joints } & Bell and spigot joints shall be caulked with oakum and lead. & \\
\hline $\begin{array}{l}\text { Transitions to Existing } \\
\text { VC }\end{array}$ & $\begin{array}{l}\text { Cast iron soil pipe, spigot ends can be joined to the hubs of } \\
\text { the same size vitrified clay. }\end{array}$ \\
\hline
\end{tabular}




\section{PIPING MATERIAL STANDARDS}

\section{Solid PVDF (Kynar) $)^{R}$, Line Class NU}

\section{Design Notes}

1. PVDF piping system shall be fabricated in strict accordance with the manufacturers recommendations and approved ICPP PVDF fusion-weld procedures.

2. Installation practices including flange bolt torque, support spacing and pipe expansion considerations shall be in compliance with the manufacturers recommendations.

3. Installed piping shall be hydrostatically tested before putting it into service in accordance with the manufacturers recommendations.

4. When assembling nonmetallic flanges, flat washers shall be used under all bolt heads and nuts.

\begin{tabular}{|c|c|c|c|}
\hline TYPE & PIPE SIZE & MATERIAL DESCRIPTION & CODE \\
\hline \multirow[b]{3}{*}{ Pipe } & $1 / 2^{\prime \prime}-2^{\prime \prime}$ & $\begin{array}{l}\text { PVDF Pipe for Socket-Welding as manufactured by } \\
\text { Resistoflex (Fluoroflex-K) }{ }^{\mathrm{R}} \text { or equal. Sch } 80 \text {. }\end{array}$ & \\
\hline & $1 / 2^{\prime \prime}-3^{\prime \prime}$ & $\begin{array}{l}\text { PVDF Pipe for Butt-Welding as manufactured by } \\
\text { ASAHI/America (Superproline) }{ }^{\mathrm{R}}, 230 \mathrm{psi} \text { Pressure Rating } \\
\text { for Water at } 73.4^{\circ} \mathrm{F} \text {. }\end{array}$ & \\
\hline & 4" - 8" & $\begin{array}{l}\text { PVDF Pipe for Butt-Welding as manufactured by } \\
\text { ASAHI/America (Superproline) }{ }^{\mathrm{R}}, 160 \text { psi Pressure Rating } \\
\text { for Water at } 73.4^{\circ} \mathrm{F} \text {. }\end{array}$ & \\
\hline \multirow[b]{3}{*}{ Fittings } & $1 / 2^{\prime \prime}-2^{\prime \prime}$ & $\begin{array}{l}\text { PVDF Fittings for Socket-Welding as manufactured by } \\
\text { Resistofle }{ }^{R} \text { or equal. Sch } 80 \text {. }\end{array}$ & \\
\hline & $1 / 2^{\prime \prime}-3^{\prime \prime}$ & \multicolumn{2}{|l|}{$\begin{array}{l}\text { PVDF Fittings for Butt-Welding as manufactured by } \\
\text { ASAHI/America (Superproline) }{ }^{\mathrm{R}}, 230 \text { psi Pressure Rating } \\
\text { for Water at } 73.4^{\circ} \mathrm{F} \text {. }\end{array}$} \\
\hline & $4 "-8 "$ & $\begin{array}{l}\text { PVDF Fittings for Butt-Welding as manufactured by } \\
\text { ASAHI/America (Superproline) }{ }^{\mathrm{R}}, 160 \text { psi Pressure Rating } \\
\text { for Water at } 73.4^{\mathrm{O}} \mathrm{F} \text {. }\end{array}$ & \\
\hline \multirow[b]{3}{*}{ Flanges } & $1 / 2^{\prime \prime}-2^{\prime \prime}$ & $\begin{array}{l}\text { PVDF Flanges, Socket-Weld, 150\# FF ANSI B16.5 } \\
\text { Dimensions as manufactured by Resistoflex }{ }^{\mathrm{R}} \text { with SST } \\
\text { Back-up Washers. }\end{array}$ & ANSI B16.5 \\
\hline & $1 / 2^{\prime \prime}-3^{\prime \prime}$ & $\begin{array}{l}\text { PVDF Stub End Butt-Weld with SST Back-up Ring as } \\
\text { manufactured by ASAHI/America (Superproline) }{ }^{\mathrm{R}}, 230 \text { psi } \\
\text { Pressure Rating for Water at } 73.4^{\circ} \mathrm{F} \text { with SST Back-up } \\
\text { Washers. }\end{array}$ & \\
\hline & $4 "-8 "$ & $\begin{array}{l}\text { PVDF Stub-End Butt-Weld with SST Back-up Ring as } \\
\text { manufactured by ASAHI/America (Superproline) }{ }^{\mathrm{R}}, 160 \mathrm{psi} \\
\text { Pressure Rating for Water at } 73.4^{\circ} \mathrm{F} \text { with SST Back-up } \\
\text { Washers. }\end{array}$ & \\
\hline \multirow{2}{*}{\multicolumn{2}{|c|}{ Gaskets }} & $\begin{array}{l}\text { Viton } \mathrm{A}^{\mathrm{R}} \text { Gaskets for } 150 \# \text { PVDF Flange, 1/8" Thick, } 60 \\
\text { Durometer. }\end{array}$ & \\
\hline & & TFE Envelope Gasket for 150\# PVDF Flange. & \\
\hline \multicolumn{2}{|c|}{ Bolts } & $\begin{array}{l}\text { Use } 304 \text { SST Bolts ASTM A193 Gr B8 and Galling } \\
\text { Resistant Nitronic } 60^{\mathrm{R}} \text { Nuts ASTM A194 Gr 8S (UNS } \\
\text { S21800). }\end{array}$ & $\begin{array}{l}\text { See Note } 4 \text { ASTM A193 Gr B } 8 \\
\text { ASTM A194 Gr 8S UNS S21800 }\end{array}$ \\
\hline
\end{tabular}




\section{PIPING MATERIAL STANDARDS}

\section{Solid PVDF (Kynar) ${ }^{R}$, Line Class NU}

\begin{tabular}{|c|l|l|l|}
\hline TYPE & PIPE SIZE & \multicolumn{1}{|c|}{ MATERIAL DESCRIPTION } & CODE \\
\hline $\begin{array}{c}\text { Gate } \\
\text { Valves }\end{array}$ & $1-1 / 2^{\prime \prime}-8^{\prime \prime}$ & $\begin{array}{l}\text { Solid PVDF, 150\# Class FF Flanged, Non-Rising Stem, } \\
\text { Seal material to be specified for particular corrosion and } \\
\text { radiation service. }\end{array}$ & \\
\hline $\begin{array}{c}\text { Check } \\
\text { Valves }\end{array}$ & $3 / 4^{\prime \prime}-8^{\prime \prime}$ & $\begin{array}{l}\text { Solid PVDF, 150\# Class FF Flanged, Horizontal Swing, } \\
\text { Seat and Seal Material to be specified for particular } \\
\text { corrosion and radiation service. }\end{array}$ & \\
\hline & $1 / 2^{\prime \prime}-6^{\prime \prime}$ & $\begin{array}{l}\text { Solid PVDF, 150\# Class FF Flanged, True-Union Type, } \\
\text { Seat and Seal Material to be specified for particular } \\
\text { corrosion and radiation service. }\end{array}$ & \\
\cline { 2 - 5 } Ball Valves & $1 / 2 "-2 "$ & $\begin{array}{l}\text { Solid PVDF, 150\# Socket-Weld, True-Union, Seat and Seal } \\
\text { Material to be specified for particular service. }\end{array}$ & \\
\hline
\end{tabular}


PIPING MATERIAL STANDARDS

\section{Carbon Steel - PVDF (Kynar) ${ }^{R}$ Lined, 150 psi Service Rating, Line Class NV}

\section{Design Notes}

1. Manufacturer's suggested bolt torque shall be used in assembling flange joints.

2. All in-cell carbon steel and forged steel piping shall be protected from decontaminant piping corrosion by epoxy coating all exposed external surfaces.

3. No gaskets are normally required between flanges with molded raised faces. Before disconnecting flanges, scribe match marks in flange sides to ensure exact line-up when reconnected.

4. The following pressure limitations shall be followed for diaphragm valves at $100^{\circ} \mathrm{F}$.

$$
\begin{array}{ll}
\frac{\text { Size }}{1 "-4 "} & \frac{\text { Max. }}{\text { Pressure }} \\
\text { 6" only } & 125 \text { psig } \\
125 \text { psig }
\end{array}
$$

\begin{tabular}{|c|c|c|c|}
\hline TYPE & PIPE SIZE & MATERIAL DESCRIPTION & CODE \\
\hline Pipe & $1 "-5 "$ & $\begin{array}{l}\text { Carbon Steel, Schedule 40, Lined with PVDF 150\# ANSI } \\
\text { B16.5 Cast Steel Flange Ends. }\end{array}$ & ASTM A587 ANSI B16.5 \\
\hline Fittings & $1 "-6 "$ & $\begin{array}{l}\text { Cast Steel Flanged Fittings, 150\# ANSI B16.5, Lined with } \\
\text { PVDF, Molded Raised Face. }\end{array}$ & $\begin{array}{l}\text { ANSI B16.5 ASTM A216 Grade } \\
\text { WCB }\end{array}$ \\
\hline \multicolumn{2}{|c|}{ Diaphragm Valves } & $\begin{array}{l}\text { 150\# Class Molded Raised Face PVDF Lined Cast Carbon } \\
\text { Steel, Hand Wheel Operated with TFE Diaphragms } \\
\text { (Outside Cell Only.) Diaphragm Material shall be specified } \\
\text { for the particular service when in-cell. }\end{array}$ & $\begin{array}{l}\text { See Note } 4 \text { ANSI B16.5 ASTM } \\
\text { A216 Grade WCB }\end{array}$ \\
\hline $\begin{array}{l}\text { Check } \\
\text { Valve }\end{array}$ & $1 "-6 "$ & $\begin{array}{l}\text { 150\# Class Molded Raised Face Cast Steel, PVDF Lined, } \\
\text { Diaphragm material shall be specified for the particular } \\
\text { service when in-cell. }\end{array}$ & $\begin{array}{l}\text { ASTM A216 Grade WCB ANSI } \\
\text { B16.5 }\end{array}$ \\
\hline $\begin{array}{c}\text { Flanges } \\
\text { Companion }\end{array}$ & $1 "-6 "$ & $\begin{array}{l}\text { 150\# ANSI B16.5, Forged Steel or Ductile Iron, Screwed, } \\
\text { Chamfered. }\end{array}$ & $\begin{array}{l}\text { See Note } 6 \text { ASTM A105 ASTM } \\
\text { A } 395 \text { ANSI B16.5 }\end{array}$ \\
\hline $\begin{array}{c}\text { Flanges } \\
\text { (Blind) }\end{array}$ & 1" - 6" & $\begin{array}{l}\text { 150\# ANSI B16.5, Forged Steel or Ductile Iron Blind } \\
\text { Flanges, (use with PVDF Full Face Blind Spacer). }\end{array}$ & $\begin{array}{l}\text { ASTM A105 ASTM A395 ANSI } \\
\text { B16.5 }\end{array}$ \\
\hline \multicolumn{2}{|c|}{ Reducing Flanges (Filler) } & $\begin{array}{l}\text { PVDF Lined 150\# ANSI B16.5, Forged Steel or Ductile } \\
\text { Iron. }\end{array}$ & $\begin{array}{l}\text { ASTM A105 ASTM A395 ANSI } \\
\text { B16.5 }\end{array}$ \\
\hline \multicolumn{2}{|c|}{ Flanges } & $\begin{array}{l}\text { Solid PVDF 2" Thickness with Stainless Steel Back-up } \\
\text { Washers. }\end{array}$ & \\
\hline \multicolumn{2}{|c|}{ Gaskets } & Viton $\mathrm{A}^{\mathrm{R}}, 1 / 16^{\prime}$ Thickness 60 Durometer & \\
\hline \multicolumn{2}{|c|}{ Bolting } & $\begin{array}{l}\text { Use } 304 \text { SST Bolts ASTM A193 Gr B8 and Galling } \\
\text { Resistant Nitronic } 60^{\mathrm{R}} \text { Nuts ASTM A194 Gr 8S (UNS } \\
\text { S21800). }\end{array}$ & $\begin{array}{l}\text { ASTM A193 Grade B8 ASTM } \\
\text { A194 Grade 8S UNS S21800 }\end{array}$ \\
\hline Branches & $1 "-6 "$ & Use Full or Reducing PVDF Lined Flanged Fittings. & \\
\hline \multicolumn{2}{|c|}{ Spacers } & $\begin{array}{l}\text { Use Standard Full-Face or Reducing Full-Face PVDF } \\
\text { Spacer of } 1 / 2 \text { " Minimum Thickness when Mating PVDF } \\
\text { Lined Piping Items with all other type of Flanged Piping. }\end{array}$ & \\
\hline
\end{tabular}

5. Companion flanges are used for molded raised face and gasketed pipe joints with PVDF liner. 
PIPING MATERIAL STANDARDS

\section{Polyvinyl Chloride (PVC), Temperature Limit 140EF, Line Class NW}

\section{$\underline{\text { Design Notes }}$}

1. When assembling nonmetallic flanges, flat washers shall be used under all bolt heads and nuts.

2. For use on cold potable water outside buildings only. Note maximum temperature for $\mathrm{PVC}$ is $140^{\circ} \mathrm{F}$. For hot or cold potable water piping inside (or outside) buildings use CPVC (Line Class ND).

3. Bronze or iron body valves may be used at designers option where corrosion is not a problem.

4. Use PVC primer and cement for solvent Welding of SW fittings. (ASTM F656 ASTM D2564)

5. See manufacturers recommendations for cleaning and joining PVC pipe and fittings.

6. This line class shall not be used in contact with or within 10' of 300 series austenitic stainless steel line classes to avoid chloride contamination.

\begin{tabular}{|c|l|l|l|}
\hline TYPE & PIPE SIZE & \multicolumn{1}{|c|}{ MATERIAL DESCRIPTION } & \multicolumn{1}{c|}{ CODE } \\
\hline Pipe & $1 / 2 "-6 "$ & PVC, Plain Ends for SW (preferred) or SCRD Schedule 80. & ASTM D1785 \\
\hline & $1 / 2 "-3 "$ & PVC Fittings, SW (preferred) or SCRD. Schedule 80. & ASTM D2464 ASTM D2467 \\
\cline { 2 - 5 } Fittings & $1 "-6 "$ & PVC Fittings, 150\# ANSI B16.5 Flanged, Schedule 80. & $\begin{array}{l}\text { Same as above } \\
\text { ANSI B16.5 }\end{array}$ \\
\hline Ball Valves & $1 / 2 "-4 "$ & PVC, SCRD, SW or Flanged. True Union Type. & See Note 3 \\
\hline Flanges & $1 / 2 "-6 "$ & PVC SW (preferred) or SCRD, 150\# ANSI B16.5 & $\begin{array}{l}\text { ASTM A2464 ASTM D2467 See } \\
\text { Note 1 ANSI B16.5 }\end{array}$ \\
\hline \multicolumn{2}{|c|}{ Gaskets } & TFE, Full Face - Non-Radiation Use Only. & \\
\hline \multicolumn{2}{|c|}{ Bolting } & Carbon Steel Bolts and Nuts. & ASTM A307 Grade B See Note 1 \\
\hline
\end{tabular}


PIPING MATERIAL STANDARDS

\section{Asbestos - Cement Per AWWA C400, Line Class NX}

\section{Design Notes}

1. This specification describes existing piping only. On future installations this material specification shall be prohibited.

\begin{tabular}{|c|c|c|c|}
\hline TYPE & PIPE SIZE & MATERIAL DESCRIPTION & CODE \\
\hline \multicolumn{2}{|c|}{ Pipe } & Asbestos - cement for water and other. & C400 AWWA \\
\hline \multicolumn{2}{|c|}{ Fittings } & $\begin{array}{l}\text { Fitting shall be mechanical joint or taper fittings consistent } \\
\text { with the manufacturer's recommendation for the piping } \\
\text { used. }\end{array}$ & \\
\hline \multicolumn{2}{|c|}{ Valves } & Refer to piping manufacturers recommendation. & \\
\hline \multicolumn{2}{|c|}{ Flanges } & Same as above. & \\
\hline \multicolumn{2}{|c|}{ Gaskets } & Same as above. & \\
\hline \multicolumn{2}{|c|}{ Bolting } & Same as above. & \\
\hline
\end{tabular}


PIPING MATERIAL STANDARDS

\section{Asbestos - Cement Per AWWA C400, Line Class NX}

$\underline{\text { Design Notes }}$

1. Use ABS solvent cement for joining pipe and fittings made to this specification. (ASTM D2235)

\begin{tabular}{|c|l|l|c|}
\hline TYPE & PIPE SIZE & \multicolumn{1}{|c|}{ MATERIAL DESCRIPTION } & \multicolumn{1}{c|}{ CODE } \\
\hline Pipe & $1-1 / 4 "-4 "$ & $\begin{array}{l}\text { Acrylonitrile - Butadiene - Styrene (ABS) Plastic Drain, } \\
\text { Waste, and Vent Pipe or ABS Sch 40 Plastic Drain, Waste, } \\
\text { and Vent Pipe with a Cellular Core. }\end{array}$ & ASTM D2661 ASTM F628 \\
\hline \multicolumn{2}{|c|}{ Fittings } & $\begin{array}{l}\text { ABS Plastic DWV Fittings SCRD or Solvent Weld ABS } \\
\text { SCH 40 Plastic DWV Fittings with a Cellular Core, SCRD } \\
\text { or Solvent Weld. }\end{array}$ & ASTM D2661 ASTM F628 \\
\hline
\end{tabular}


Project Title: TMI-2 FUEL VACUUM DRYER SPECIFICATION

Document Type: Procurement Specification Project Number: N/A

SPC: 287 


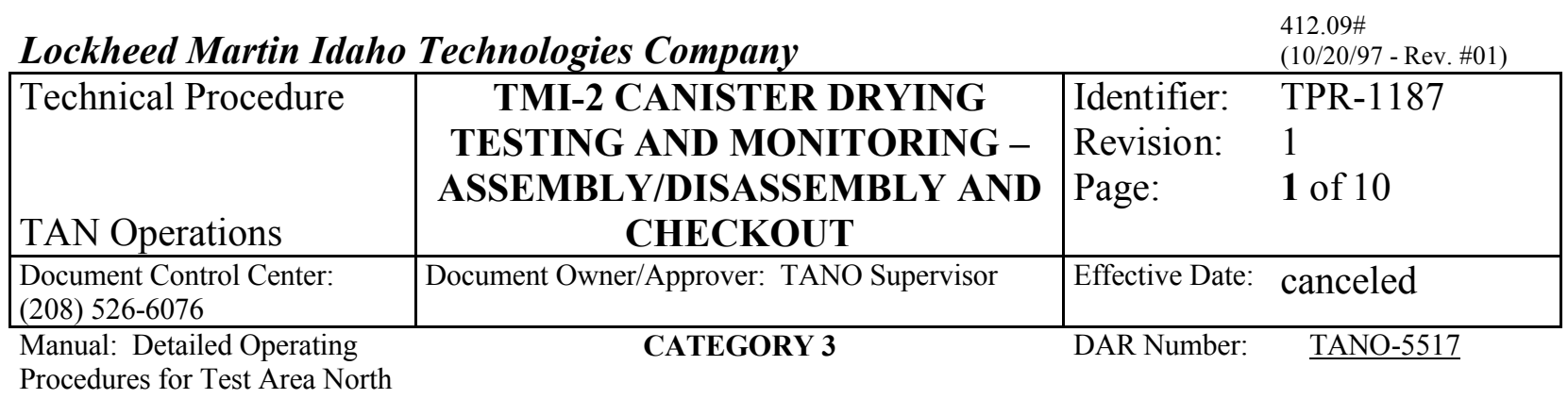

Validation - This document must be validated prior to first use by one of the following:

[ ] Walkthrough

[ ] Using mockup or simulators
[ ] Tabletop validation by safety committee and craftsmen

$[\sqrt{ }]$ First time use of procedure 


\begin{tabular}{|l|c|ll|}
\hline Technical Procedure & TMI-2 CANISTER DRYING & Identifier: & TPR-1187 \\
& TESTING AND MONITORING - & Revision: & 1 \\
PSSEMBLY/DISASSEMBLY AND & Page: & $\mathbf{2}$ of 10 \\
TAN Operations & CHECKOUT & & \\
\hline
\end{tabular}

REVISION LOG

\begin{tabular}{|c|c|c|c|}
\hline Rev. & Date & $\begin{array}{c}\text { Affected } \\
\text { Pages }\end{array}$ & Revision Descriptior \\
\hline 0 & 03/10/98 & All & Initial release. See DAR TANO-5451. \\
\hline 1 & $05 / 26 / 98$ & Various & See DAR TANO-5517. \\
\hline
\end{tabular}




\begin{tabular}{|l|c|ll|}
\hline Technical Procedure & $\begin{array}{c}\text { TMI-2 CANISTER DRYING } \\
\text { TESTING AND MONITORING - } \\
\text { TAN Operations }\end{array}$ & $\begin{array}{l}\text { Identifier: } \\
\text { Revision: }\end{array}$ & $\begin{array}{l}\text { TPR-1187 } \\
\text { ASSEMBLY/DISASSEMBLY AND } \\
\text { Page: }\end{array}$ \\
\hline
\end{tabular}

\section{INTRODUCTION}

\subsection{Purpose}

To provide instructions to assemble/disassemble and checkout the basic operation of the Heated Vacuum Drying System (HVDS) to be used to dry the TMI-2 canisters.

\subsection{Scope and Applicability}

The procedure provides HVDS testing operation within specified parameters including electrical, instrumentation, fluid transfer, and mechanical interfaces to existing systems and equipment. These interfaces include the existing dewatering system (DWS) and remote handling equipment. Checkout of the basic operation of the HVDS will ensure that all equipment is installed and aligned correctly such that testing and operation can be performed safely. The HVDS consists of the Vacuum Pump Skid (VPS), the Vacuum Condenser Skid (VCS), and the Vacuum Furnace (VF) (see Definitions).

This procedure applies only to those persons performing the HVDS assembly/disassembly or checkout.

\section{PRECAUTIONS AND LIMITATIONS}

2.1 All high temperature test equipment exposed to personnel during operations is clearly marked and proper precautions are taken when the equipment is approached.

2.2 The VF pressure relief valve (PRV3) and discharge line are clear of obstructions and connected to the DWS holding tank.

\section{PREREQUISITES}

\subsection{Planning and Coordination}

3.1.1 Hoisting and rigging equipment load test is current.

3.1.2 Equipment operators are qualified and current.

3.1.3 Personnel performing this procedure are HVDS trained.

\subsection{Performance Documents}

None

\subsection{Special Tools, Equipment, Parts, and Supplies}

\subsubsection{The equipment and materials given in Table 3.3.1 are available.}

Table 3.3.1. Equipment List

\begin{tabular}{|l|c|l|}
\hline Item & No. & Comments \\
\hline Dewatering Skid & 1 & \\
\hline $\begin{array}{l}\text { Heated Vacuum } \\
\text { Drying System }\end{array}$ & 1 & \\
\hline Vacuum Furnace & 1 & Positioned in the shielding cask \\
\hline
\end{tabular}




\begin{tabular}{|l|c|ll|}
\hline Technical Procedure & TMI-2 CANISTER DRYING & Identifier: & TPR-1187 \\
& TESTING AND MONITORING - & Revision: & 1 \\
Page: & $\mathbf{4}$ of 10 \\
TAN Operations & ASSEMBLY/DISASSEMBLY AND & CHECKOUT & \\
\hline
\end{tabular}

\begin{tabular}{|l|c|l|}
\hline Item & No. & Comments \\
\hline Shielding Cask & 1 & The internals are removed \\
\hline Bucket(s) & $1-4$ & With a total capacity of 20 gallons \\
\hline Hose Assemblies & 17 & The Hose Checklist is provided in Appendix B \\
\hline Helium Supply & 1 & To be used to leak test the system \\
\hline
\end{tabular}

3.3.2 The equipment is positioned as shown in the appropriate drawing listed below (obtained from the Test Engineer):

- Dwg. No. DWS SK-007, "Warm Shop Functional Test Layout."

- Dwg. No. DWS SK-011, "Layout Configuration for "Cold" S. O. Testing \& TAN Operator Training."

- Dwg. No. DWS SK-012, "Layout Configuration for Canister Drying Test TAN Operator Training."

\subsubsection{The instruments shown in Table 3.3.2 have a current calibration.}

Table 3.3.2 Instrument List

\begin{tabular}{|l|c|l|}
\hline Item & No. & Comments \\
\hline $\begin{array}{l}\text { Temperature Sensors - Two on the VF (TIT1 \& } \\
\text { TIT2) and one on the common drain line (TI1) }\end{array}$ & 3 & All remotely indicated except TI1 \\
\hline $\begin{array}{l}\text { Pressure Sensors - Two on the VF (PI5 \& PI6), } \\
\text { and one on the common drain line (PI3), and } \\
\text { one to provide system pressure (PI2) }\end{array}$ & 4 & $\begin{array}{l}\text { All but PI2 remotely indicated - PI5 \& PI6 provide input } \\
\text { for automatic shutoff based on over pressurization of the } \\
\text { VF }\end{array}$ \\
\hline He Detector $\left(10^{-7} \mathrm{~atm}-\mathrm{cc} / \mathrm{sec}\right)$ & 1 & Used to detect leakage of the HVDS \\
\hline
\end{tabular}

\section{Preparations}

Test Engineer: Conduct a briefing with the test personnel and complete the following items:

A. A discussion of safety precautions and emergency actions associated with the operation of the HVDS.

B. A review of Section 4 of this test procedure.

C. The table, Test Personnel Identification, in Appendix H.

Test Engineer

Date

\subsection{Approval and Notifications}

Review this procedure. Sign space indicated and enter date for approval of work to commence.

TANO Shift Supervisor: Date

\section{INSTRUCTIONS}

\subsection{Assembly}

4.1.1 Test Engineer: Ensure the prerequisites for this procedure have been completed.

4.1.2 Operations Technician (OP): Ensure the VF is seated completely into the shielding cask. 


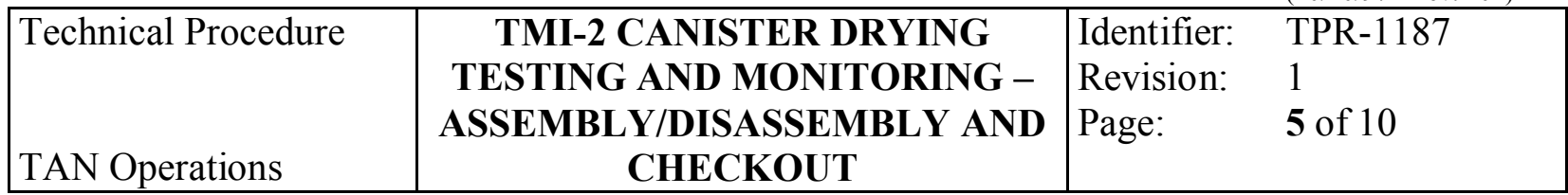

4.1.3 Complete hose connections as follows:

A. Complete the DWS Valve Checklist provided in Appendix G of this procedure.

B. Connect the 17 hoses identified in the Hose Checklist provided in Appendix B.

C. Connect Hose $1 / 2-H A D-03$ to a filtered shop air source of 80 psig or greater.

D. When Hose $1 \boldsymbol{H A D}-03$ is connected to DWS Line $3 / 4 \boldsymbol{T}$-CDS-012, open DWS V-34 (HVDS Condensate Interface Isolation Valve).

E. $\quad$ 4.1.3.1 Visually inspect all hose connections to confirm that the correct connection have been made; initial the Hose Checklist for each connection as it is verified.

4.1.4 Elect: Attach the following HVDS interconnecting electrical cables:

A. Attach $480 \mathrm{VAC}$ cables (2) from the VF to the VCS.

B. Attach the temperature probe cables from the VF to the appropriate connection in the VCS control panel.

C. Attach cables (3) from the VCS to the VPS.

4.1.5 Attach the cable from the VCS to its connection on the remote controller.

\section{CAUTION}

Check phases of 480 power to VPS and VCS to ensure proper pump and motor rotation.

4.1.6 Attach the electrical cables to the shop supply as follows:

A. Ensure proper voltage of the supply source ( 480 volt +0 to $-10 \%, 60 \mathrm{amp}$ ).

B. Ensure that cables 1 and 2 are connected to independent electrical sources.

C. Attach cable 1 to the electrical supply source (VPS cable).

D. Attach cable 2 to the electrical supply source (VCS cable).

E. Attach cable 3 to a $120 \mathrm{VAC}$ receptical (PLC power cord).

F. Open VPS and VCS control panel doors and energize panels by closing all circuit breakers.

4.1.7 Tech: Initiate the flow of regulated shop air to the HVDS by opening the Warm Shop or Hot Shop, as appropriate, air-supply valve.

4.1.8 Visually check the level of DWS Holding Tank; IF the level is not near the bottom of the sight glass, THEN use the procedure given in Appendix F, DWS Holding Tank Sampling and Pump Out, to transfer the tank contents.

4.1.9 Align switches (leave the vacuum pump switch in the Off position) to the manual operation position (Appendix D Switch Checklist). 


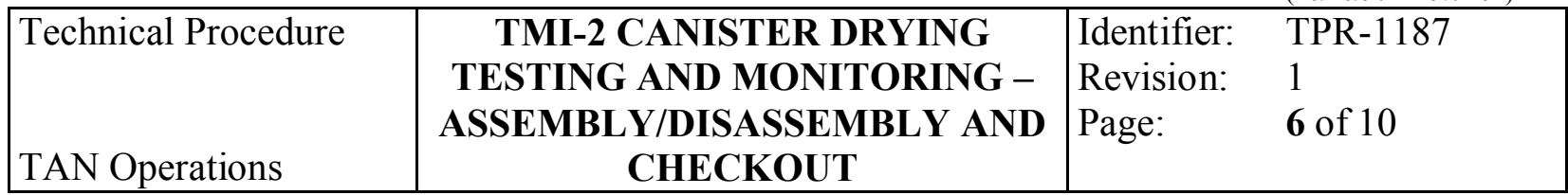

4.1.13 VF head sweep system checkout.

A. Start the head sweep system.

B. Measure airflow around the VF head (minimum of $120 \mathrm{ft} / \mathrm{min}$ ).

C. Stop the head sweep system.

4.1.14 Check instrument installation and readability of the following:

A. Pressure gauge PI1 (system pressure, 30-0-30 psig on VPS)

B. Pressure gauge PI2 (system pressure, 0-100 torr on VPS)

C. Pressure sensor PI3 (remote indication, 0-30 psig drain line pressure sensor is located on VCS)

D. Pressure sensor PI5 (remote indication, 100-0 torr VF vacuum on VCS)

E. Pressure sensor PI6 (remote indication, 100-0 torr VF vacuum on VCS)

F. Temperature sensor TIT1 (remote indication, top of canister in VCS)

G. Temperature sensor TIT2 (remote indication, bottom of canister in VCS)

H. Temperature sensor TI1 (common drain line on VCS)

I. Sight level gauge LI-1 (dewatering tank level on the DWS)

J. Programmable controller (remote installation)

\subsubsection{Acceptance Criteria}

A. The VF is seated completely into the shielding cask.

B. Hose and cable connections were correctly made to the proper locations and where applicable to the proper voltage.

C. Switches are aligned to the manual operation position.

D. Valves are aligned to the normal operation position (except V1 and V7 are in the $\boldsymbol{O} f \boldsymbol{f}$ position and V3 is opened).

E. At least one hour of vacuum pump warm-up operation has been completed.

F. Instruments are installed and readable.

G. The airflow around the VF head during head-sweep system operation was at least $120 \mathrm{ft} / \mathrm{min}$.

4.1.16 The assembly of the HVDS has been completed and met the acceptance criteria.

Test Engineer

Quality Inspector
Date

Date

\subsection{Helium Leak Test}

4.2.1 OP: Attach a suitable $\left(10^{-7} \mathrm{~atm}-\mathrm{cc} / \mathrm{sec}\right)$ helium detector to the $\mathrm{He} / \mathrm{N}_{2}$-supply port of V4 on the VPS.

4.2.2 Unseal and reseal the VF head as follows:

A. Open the VF head (press the remote Open/Close button).

B. Lift the VF head slightly.

C. Lower the VF head into position.

D. Close the VF head (press Open/Close button).

4.2.3 Manually evacuate the heated vacuum drying system as follows:

A. Open V1 on the VCS (V1 switch to the Manual position).

B. Open V7 on the VPS (V7 switch to the Manual position). 


\begin{tabular}{|l|c|ll|}
\hline Technical Procedure & $\begin{array}{c}\text { TMI-2 CANISTER DRYING } \\
\text { TESTING AND MONITORING - }\end{array}$ & $\begin{array}{ll}\text { Identifier: } \\
\text { Revision: }\end{array}$ & $\begin{array}{l}\text { TPR-1187 } \\
\text { TAge: }\end{array}$ \\
TSSEMBLY/DISASSEMBLY AND Operations & CHECKOUT & 7 of 10 \\
\hline
\end{tabular}

C. Evacuate the system until a vacuum of $<0.5$ torr is indicated on the VF gauges (PI5 and PI6) and the system pressure gauge (PI2).

4.2.4 Close V7 (V7 switch to the Off position).

\subsubsection{Open V4.}

4.2.6 Flood critical areas of the HVDS one at a time with helium [include at a minimum all hose connections (Appendix B), instrumentation to piping attachment points and closure of the VF head].

4.2.7 Use the helium detector connected to the helium supply port to monitor for helium leaks.

4.2.8 IF leakage is identified THEN, seal and/or repair all leaks and repeat steps 4.4.2 to 4.4.7.

4.2.9 Close V4 and remove the helium detector.

\subsubsection{Acceptance Criteria}

\subsubsection{No leakage is detected.}

4.2.11 The helium leak testing is completed without detecting leakage.

Test Engineer

Quality Inspector
Date

Date

\subsection{Vacuum Leak Test}

4.3.1 OP: Manually evacuate the heated vacuum drying system as follows:

A. Openlensure-open V1 on the VCS (V1 switch to the Manual position).

B. Open V7 (V7 switch to the Manual position).

C. WHEN the vacuum is $<0.5$ torr, THEN:

a. Close V7 (V7 switch to the Off position).

b. Close V1 (V1 switch to the $\boldsymbol{O} f \boldsymbol{f}$ position).

c. Monitor pressure from PI2, PI5, and PI6 to identify unacceptable leakage (inability to maintain at least 1.0 torr for 30 minutes).

D. IF unacceptable leakage occurs THEN, pressurize the system as given below, seal and/or repair all leaks, and repeat Step 4.5.1.

E. It is probable that some leakage will occur, if practicable determine the system leak rate $(\Delta \mathrm{P} /$ time $)$.

4.3.2 Pressurize the system as follows:

A. Open V1 (V1 switch to the Manual position).

B. Open V11.

C. WAIT until atmospheric pressure is restored THEN, unseal and reseal the VF head as follows:

a. Open the VF head (press the remote Open/Close button).

b. Lift the VF head slightly.

c. Lower the VF head into position.

d. Close the VF head (press the remote Open/Close button). 


\begin{tabular}{|l|c|ll|}
\hline Technical Procedure & $\begin{array}{c}\text { TMI-2 CANISTER DRYING } \\
\text { TESTING AND MONITORING - } \\
\text { TAN Operations }\end{array}$ & $\begin{array}{l}\text { Identifier: } \\
\text { Revision: }\end{array}$ & $\begin{array}{l}\text { TPR-1187 } \\
\text { ASSEMBLY/DISASSEMBLY AND } \\
\text { Page: }\end{array}$ \\
\hline
\end{tabular}

D. Close V11.

\subsubsection{Acceptance Criteria}

\subsubsection{HVDS maintains a vacuum of at least 1.0 torr for 30 minutes.}

4.3.4 The vacuum leak testing is completed and met the acceptance criteria.

Test Engineer

Quality Inspector
Date

Date

\subsection{Automatic (normal) Operation Test}

4.4.1 OP: Position the HVDS switches to the Normal Operation positions (Appendix D).

4.4.2 Align valves to the normal operation position (Appendix C Valve Checklist).

4.4.3 Press the remote Start button to begin automatic operation.

4.4.4 Record temperature and pressure data in Test Log (Appendix E) at 15-minute increments UNTIL the system shuts down and the PLC temperature indicated a decay.

\subsubsection{Acceptance Criteria}

When the remote Start button is pressed, the HVDS automatically begins the vacuum and heating process, senses drying completion, performs the Acceptance Test ( $<80$ torr for 30 minutes) and shuts down the system.

4.4.6 The automatic operation performed as expected and met the acceptance criteria.

Test Engineer

Quality Inspector
Date

Date

\subsection{High-Pressure Automatic-Shutdown Test}

4.5.1 OP: Pressurize the system and load with water as follows:

A. Open V1 (V1 switch to the Manual position).

B. Open V4.

C. WAIT until atmospheric pressure is restored THEN, unseal, add buckets, and reseal the VF head as follows:

a. Open the VF head (press the Open/Close button).

b. Lift the VF head until it rests against the hinge stops.

c. Place about 20 gallons (1 -4 buckets) of demineralized water into the VF.

d. Lower the VF head into position.

e. Close the VF head (press Open/Close the button).

D. Close V4.

E. Switch V1 to the Automatic position. 


\begin{tabular}{|l|c|ll|}
\hline Technical Procedure & $\begin{array}{c}\text { TMI-2 CANISTER DRYING } \\
\text { TESTING AND MONITORING - } \\
\text { TAN Operations }\end{array}$ & $\begin{array}{ll}\text { Identifier: } \\
\text { Revision: }\end{array}$ & $\begin{array}{l}\text { TPR-1187 } \\
\text { ASSEMBLY/DISASSEMBLY AND } \\
\text { Page: }\end{array}$ \\
\hline
\end{tabular}

4.5.2 Visually check the level of dewatering holding tank; $\underline{I F}$ the level is not at least seven inches below the Upper alarm sensor, THEN use the procedure given in Appendix F, DWS Holding Tank Sampling and Pump Out, to transfer the tank contents.

4.5.3 Press the remote Start Button.

4.5.4 Record temperature and pressure data in Test Log (Appendix E) at 15-minute increments UNTIL the PLC temperature reaches $>200^{\circ} \mathrm{F}$, THEN manually close V1 (V1 switch to the $\boldsymbol{O f f}$ position) to simulate the loss of vacuum.

4.5.5 CONTINUE to collect data UNTIL the system reduces temperature automatically when the upper pressure limit of 100 torr is reached (if possible).

4.5.6 IF the system does not automatically begin to reduce temperature prior to it reaching $900^{\circ} \mathrm{F}$, THEN press the remote Stop button.

4.5.7 Record temperature and pressure data in the Test Log at 15-minute increments UNTIL a positive indication of temperature decay is achieved.

4.5.8 Reset V1 to the automatic position and complete the drying process.

4.5.9 Pressurize the system and remove the buckets as follows:

A. Open V1 (V1 switch to the Manual position).

B. Open V4.

C. WAIT until atmospheric pressure is restored THEN, unseal, remove buckets, and reseal the VF head as follows:
a. Open the VF head (press the Open/Close button).
b. Lift the VF head until it rests against the hinge stops.
c. Remove the bucket(s)
d. Lower the VF head into position.
e. Close the VF head (press Open/Close the button).

D. Close V4.

E. Switch V1 to the Automatic position.

\subsubsection{Acceptance Criteria}

The system automatically reduces temperature when the upper pressure limit of 100 torr is reached.

4.5.11 The system operated as expected and met the acceptance criteria.

Test Engineer
Date

Date

\subsection{Disassembly}

4.6.1 OP: Shut off the flow of regulated shop air from the HVDS by closing the Warm Shop or Hot Shop, as appropriate, air-supply valve.

4.6.2 If the vacuum pump is running, stop it (Stop button).

4.6.3 EL: Complete the electrical detachments from the electrical supply as follows: 


\begin{tabular}{|c|c|c|c|}
\hline Гechnical Procedure & $\begin{array}{c}\text { TMI-2 CANISTER DRYING } \\
\text { TESTING AND MONITORING - } \\
\text { ASSEMBLY/DISASSEMBLY AND } \\
\text { CHFCKOUT }\end{array}$ & $\begin{array}{l}\text { Identifier: } \\
\text { Revision: } \\
\text { Page: }\end{array}$ & $\begin{array}{l}\text { TPR-1187 } \\
1 \\
\mathbf{1 0} \text { of } 10\end{array}$ \\
\hline
\end{tabular}
A. Detach cable 1 from the electrical supply source.
B. Detach cable 2 from the electrical supply source.
C. Detach cable 3 from electrical supply source.

4.6.4 Detach the remote controller cable from its connection on the VCS.

4.6.5 Detach the following HVDS interconnecting electrical cables:
A. Detach cables from the VF to the VCS.
B. Detach cables from the VF to the VPS.
C. Detach cables from the VCS to the VPS.

4.6.6 Tech: Detach hose connections as follows:

A. Disconnect the 17 hoses identified in the Hose Checklist (Appendix B).

B. WHEN Hose 1-HAD-03 is disconnected from DWS Line $3 / 4 \boldsymbol{T}$-CDS-012, THEN close DWS V-34 (HVDS Condensate Interface Isolation Valve).

\section{POST PERFORMANCE ACTIVITIES}

None

6. RECORDS

NOTE: Records will be kept in the project file.

\begin{tabular}{|c|c|c|}
\hline Record & Retention & Disposition \\
\hline $\begin{array}{c}\text { This procedure, including all data } \\
\text { sheets when completed. }\end{array}$ & Permanent & Destroy when no longer needed. \\
\hline
\end{tabular}

\section{SOURCE REQUIREMENTS}

See Appendix J for procedure basis.

\section{APPENDICES}

Appendix A, Figure

Appendix B, Hose Checklist

Appendix C, HVDS Valve Checklist

Appendix D, Switch Checklist

Appendix E, Drying Test Log

Appendix F, DWS Sampling and Pump Out

Appendix G, DWS Valve Checklist

Appendix H, Test Personnel Identification

Appendix I, Definitions

Appendix J, Procedure Basis 


\begin{tabular}{|c|c|c|c|}
\hline Technical Procedure & $\begin{array}{c}\text { TMI-2 CANISTER DRYING } \\
\text { TESTING AND MONITORING - } \\
\text { ASSEMBLY/DISASSEMBLY AND } \\
\text { CHECKOUT }\end{array}$ & $\begin{array}{l}\text { Identifier: } \\
\text { Revision: } \\
\text { Page: }\end{array}$ & $\begin{array}{l}\text { TPR-1187 } \\
1 \\
\text { A1 of A1 }\end{array}$ \\
\hline
\end{tabular}

\section{APPENDIX A}

\section{Figure}

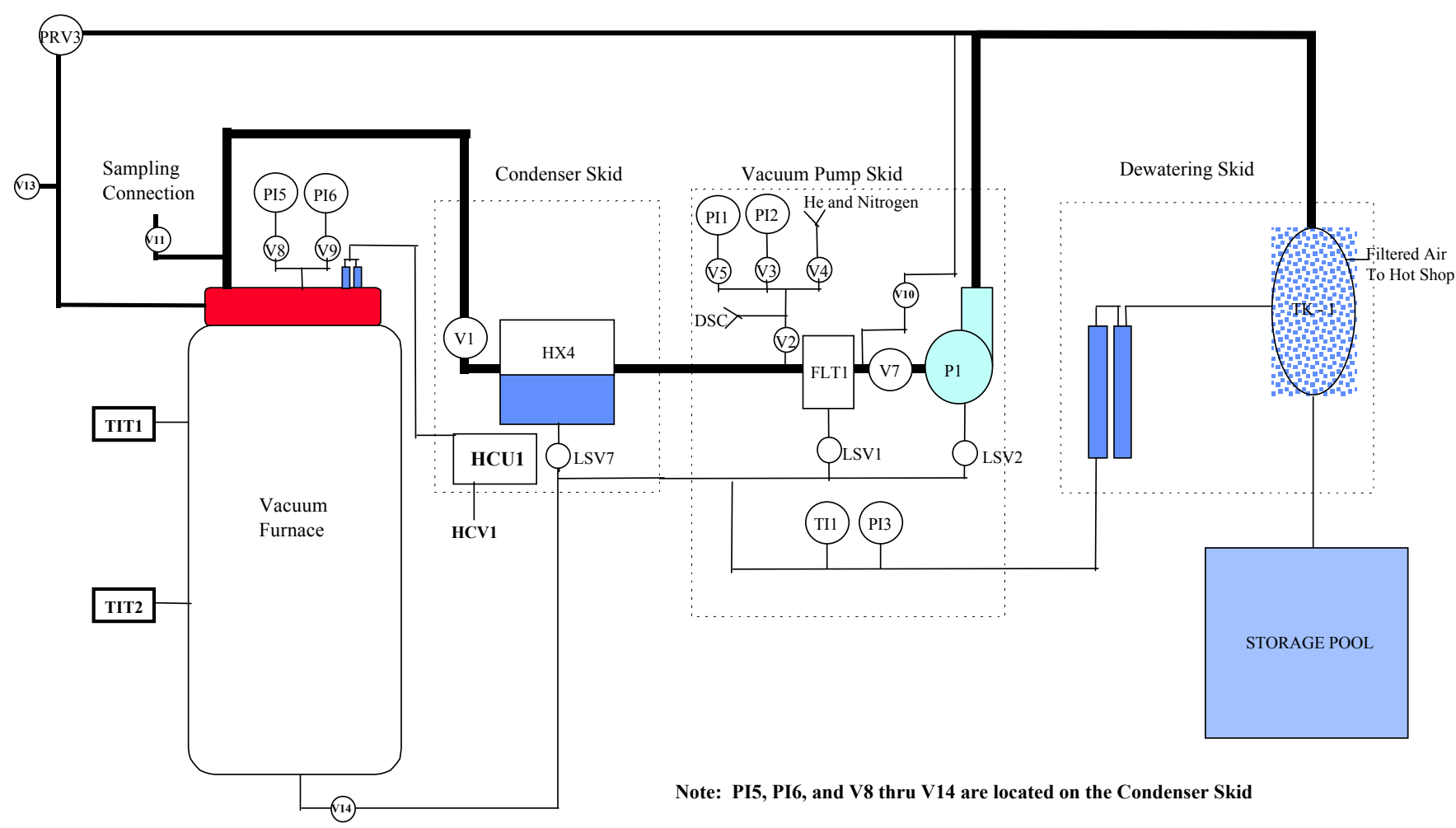

Figure 1. Heated Vacuum Drying System (HVDS) Schematic. 


\begin{tabular}{|c|c|c|c|}
\hline Technical Procedure & $\begin{array}{c}\text { TMI-2 CANISTER DRYING } \\
\text { TESTING AND MONITORING - } \\
\text { ASSEMBLY/DISASSEMBLY AND } \\
\text { CHECKOUT }\end{array}$ & $\begin{array}{l}\text { Identifier: } \\
\text { Revision: } \\
\text { Page: }\end{array}$ & $\begin{array}{l}\text { TPR-1187 } \\
1 \\
\text { B1 of B1 }\end{array}$ \\
\hline
\end{tabular}

\section{APPENDIX B}

Hose Checklist

Procedure Step

Assembly/Disassembly Dates(s)

Location

\begin{tabular}{|c|c|c|c|c|c|c|c|c|c|c|c|}
\hline \multirow[t]{2}{*}{ Order } & \multirow[t]{2}{*}{$\begin{array}{l}\text { Hose } \\
\text { Assembly } \\
\text { Diagram \# }\end{array}$} & \multicolumn{2}{|c|}{$\begin{array}{c}\text { Vacuum Furnace } \\
\text { Skid (VFS) }\end{array}$} & \multicolumn{2}{|c|}{$\begin{array}{c}\text { Vacuum } \\
\text { Condenser } \\
\text { Skid (VCS) } \\
\end{array}$} & \multicolumn{2}{|c|}{$\begin{array}{c}\text { Vacuum Pump } \\
\text { Skid (VPS) }\end{array}$} & \multicolumn{2}{|c|}{ Other Systems } & \multirow[t]{2}{*}{ Initials } & \multirow[t]{2}{*}{$\begin{array}{c}\text { Independent } \\
\text { Verification } \\
\end{array}$} \\
\hline & & $\begin{array}{l}\text { VFS- } \\
\text { CPL }\end{array}$ & $\begin{array}{l}\text { HAD- } \\
\text { CPL }\end{array}$ & $\begin{array}{l}\text { VCS- } \\
\text { CPL }\end{array}$ & $\begin{array}{l}\text { HAD- } \\
\text { CPL }\end{array}$ & $\begin{array}{l}\text { VPS- } \\
\text { CPL }\end{array}$ & $\begin{array}{l}\text { HAD- } \\
\text { CPL }\end{array}$ & SYSTEM & $\begin{array}{l}\text { HAD- } \\
\text { CPL }\end{array}$ & & \\
\hline 1 & 2-HAD-02 & -01 & -03 & -01 & -04 & & & & & & \\
\hline 2 & 3/4-HAD-01 & -04 & -09 & -06 & -10 & & & & & & \\
\hline 3 & 1/2-HAD-07 & -05 & -27 & -09 & -28 & & & & & & \\
\hline 4 & 1/2 HAD-06 & -06 & -26 & -10 & -25 & & & & & & \\
\hline 5 & 1/2-HAD-08 & -07 & -29 & -08 & -30 & & & & & & \\
\hline 6 & 1/2-HAD-09 & -08 & -31 & -12 & -32 & & & & & & \\
\hline 7 & 1/2-HAD-10 & -09 & -33 & -13 & -34 & & & & & & \\
\hline 8 & 1-HAD-01 & -02 & -08 & & & -01 & -07 & & & & \\
\hline 9 & 1/4-HAD-04 & & & -02 & -21 & -09 & -22 & & & & \\
\hline 10 & 2-HAD-01 & & & -03 & -01 & -04 & -02 & & & & \\
\hline 11 & 1/2-HAD-02 & & & -04 & -17 & -02 & -18 & & & & \\
\hline$* 12$ & 3/4-HAD-02 & & & -05 & -11 & -08 & -12 & & & & \\
\hline 13 & 1-HAD-02 & & & -14 & -13 & & & DWS & -14 & & \\
\hline 14 & 11/2-HAD-01 & & & & & -06 & -05 & DWS & -06 & & \\
\hline 15 & 1/2-HAD-03 & & & & & -03 & -20 & Shop Air & -19 & & \\
\hline 16 & 1/2-HAD-05 & & & & & -07 & -24 & $\mathrm{He} / \mathrm{N}_{2}$ & -23 & & \\
\hline 17 & 1-HAD-03 & & & & & -10 & -35 & DSC & -36 & & \\
\hline
\end{tabular}

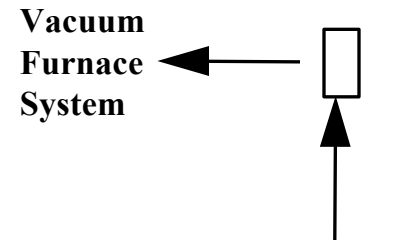

VFS-CPL-01 HAD-CPL-03

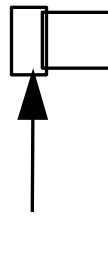

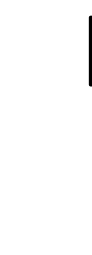

(a)

\section{CONNECTION EXAMPLE}

* Hose 12 it not to be connected. The condensate outlet at valve V15 is to be routed to an independent container to check for oily residue. 


\begin{tabular}{|c|c|c|c|}
\hline Technical Procedure & $\begin{array}{c}\text { TMI-2 CANISTER DRYING } \\
\text { TESTING AND MONITORING - } \\
\text { ASSEMBLY/DISASSEMBLY AND } \\
\text { CHECKOUT }\end{array}$ & $\begin{array}{l}\text { Identifier: } \\
\text { Revision: } \\
\text { Page: }\end{array}$ & $\begin{array}{l}\text { TPR-1187 } \\
1 \\
\text { C1 of } 11\end{array}$ \\
\hline
\end{tabular}

APPENDIX C

HVDS Valve Checklist

Procedure Step

Test Name Test Dates Test Number

\begin{tabular}{|c|c|c|c|c|c|}
\hline $\begin{array}{l}\text { Valve } \\
\text { Number }\end{array}$ & $\begin{array}{l}\text { Normal } \\
\text { Operation }\end{array}$ & $\begin{array}{l}\text { Acceptance } \\
\text { Testing }\end{array}$ & $\begin{array}{l}\text { Furnace } \\
\text { Pressurization }\end{array}$ & Vacuum Furnace Sampling & Initials \\
\hline $\mathrm{V} 1$ & Opened & Closed & Opened & Closed & \\
\hline V2 & Opened & Opened & Opened & Opened & \\
\hline $\mathrm{V} 3$ & Closed & Closed & Closed & Closed & \\
\hline $\mathrm{V} 4$ & Closed & Closed & Closed & Closed & \\
\hline V5 & Opened & Opened & Opened & Opened & \\
\hline $\mathrm{V7}$ & Opened & Closed & Closed & Closed & \\
\hline V8 & Opened & Opened & Closed & Closed & \\
\hline V9 & Opened & Opened & Closed & Closed & \\
\hline V10 & Closed & Closed & Closed & Closed & \\
\hline V11 & Closed & Closed & Closed & Opened & \\
\hline V14 & Closed & Closed & Closed & May be used to purge furnace & \\
\hline *V15 & Opened & Opened & Opened & Opened & \\
\hline V16 & Closed & Closed & Closed & $\begin{array}{l}\text { Normally Closed (Handle } \\
\text { Removed) }\end{array}$ & \\
\hline V17 & & & & Preset (Handle Removed) & \\
\hline V18 & Closed & Closed & Closed & Closed & \\
\hline \multicolumn{6}{|c|}{$\begin{array}{l}\text { Notes: } \\
\text { 1. Valves are identified in Appendix A Figure } 1 . \\
\text { 2. Ensure that the Air Sampler is installed prior to opening valve V11 when performing "Canister Testing" } \\
\text { 3. Valve indication lights are illuminated when valves are opened. }\end{array}$} \\
\hline $\begin{array}{l}\mathrm{V} \\
\mathrm{lo}\end{array}$ & \multicolumn{5}{|c|}{$\begin{array}{l}\text { Valve V15 is to be opened for this test. The condensate from the VPS is to be routed to a separate } \\
\text { location to check for oily residue. }\end{array}$} \\
\hline
\end{tabular}




\begin{tabular}{|l|c|ll|}
\hline Technical Procedure & $\begin{array}{c}\text { TMI-2 CANISTER DRYING } \\
\text { TESTING AND MONITORING - } \\
\text { TSSEMBLY/DISASSEMBLY AND } \\
\text { TAN Operations }\end{array}$ & $\begin{array}{l}\text { Identifier: } \\
\text { Revision: }\end{array}$ & $\begin{array}{l}\text { TPR-1187 } \\
\text { Page: }\end{array}$ \\
\hline
\end{tabular}

APPENDIX D

Switch Checklist

Procedure Step

Test Name Test Dates Test Number

\begin{tabular}{|l|l|l|l|l|l|l|}
\hline \multicolumn{1}{|c|}{$\begin{array}{l}\text { Switch } \\
\text { Name }\end{array}$} & $\begin{array}{l}\text { Switch } \\
\text { Location }\end{array}$ & $\begin{array}{l}\text { Switch } \\
\text { Label }\end{array}$ & $\begin{array}{l}\text { Normal } \\
\text { Operation } \\
\text { (Automatic) }\end{array}$ & $\begin{array}{l}\text { Manual } \\
\text { Operation }\end{array}$ & $\begin{array}{l}\text { Off } \\
\text { Position }\end{array}$ & Initial \\
\hline & & & & & & \\
\hline $\begin{array}{l}\text { Coolant Circulation } \\
\text { Motor }\end{array}$ & VCS & Coolant Pump & On & On & Off & \\
\hline $\begin{array}{l}\text { Refrigeration } \\
\text { System Motor }\end{array}$ & VCS & Refr System & Auto & Manual & Off & \\
\hline Valve V1 Operator & VCS & V1 & Auto & Manual & Off & \\
\hline & & & & & & \\
\hline Heater 1 & VCS & Heat Bank 1 & Auto & Off & NA $^{*}$ & \\
\hline Heater 2 & VCS & Heat Bank 2 & Auto & Off & NA $^{*}$ & \\
\hline $\begin{array}{l}\text { Hydraulic Closer } \\
\text { Operation }\end{array}$ & VCS & Vessel Open & $\begin{array}{l}\text { Open or Closed } \\
\text { as appropriate }\end{array}$ & $\begin{array}{l}\text { Open or Closed } \\
\text { as appropriate }\end{array}$ & Off & \\
\hline & --Closed & & & & \\
\hline Oil Pump & VPS & Oil Pump & Auto & Manual & Off & \\
\hline Vacuum Pump & VPS & Vacuum Pump & Auto & Manual & Off & \\
\hline Valve V7 Operator & VPS & V7 & Auto & Manual & Off & \\
\hline
\end{tabular}

${ }^{*} \mathrm{NA}=$ Not Applicable 


\begin{tabular}{|l|c|ll|}
\hline Technical Procedure & $\begin{array}{c}\text { TMI-2 CANISTER DRYING } \\
\text { TESTING AND MONITORING - } \\
\text { TAN Operations }\end{array}$ & $\begin{array}{ll}\text { Identifier: } \\
\text { Revision: } \\
\text { ASSEMBLY/DISASSEMBLY AND }\end{array}$ & $\begin{array}{l}\text { TPR-1187 } \\
\text { Page: }\end{array}$ \\
\hline
\end{tabular}

APPENDIX E

Drying Test Log

Procedure Step

Test Name

Test Dates Test Number

\begin{tabular}{|c|c|c|c|c|c|c|c|c|c|}
\hline $\begin{array}{l}\text { TIME } \\
(\mathrm{min})\end{array}$ & $\begin{array}{c}\text { PLC } \\
\text { TEMP } \\
\left({ }^{\mathbf{0}} \mathrm{F}\right) \\
\end{array}$ & $\begin{array}{l}\text { TT1 } \\
\left({ }^{\mathbf{0}} \mathbf{F}\right) \\
\end{array}$ & $\begin{array}{l}\text { TI1 } \\
\left({ }^{0} \mathbf{F}\right) \\
\end{array}$ & $\begin{array}{l}\text { PI3 } \\
\text { (psi) }\end{array}$ & $\begin{array}{c}\text { PI5 } \\
\text { (torr) }\end{array}$ & $\begin{array}{c}\text { PI6 } \\
\text { (torr) }\end{array}$ & $\mathrm{CV}$ & INITIAL & COMMENTS \\
\hline & & & & & & & & & \\
\hline & & & & & & & & & \\
\hline & & & & & & & & & \\
\hline & & & & & & & & & \\
\hline & & & & & & & & & \\
\hline & & & & & & & & & \\
\hline & & & & & & & & & \\
\hline & & & & & & & & & \\
\hline & & & & & & & & & \\
\hline & & & & & & & & & \\
\hline & & & & & & & & & \\
\hline & & & & & & & & & \\
\hline & & & & & & & & & \\
\hline & & & & & & & & & \\
\hline & & & & & & & & & \\
\hline & & & & & & & & & \\
\hline & & & & & & & & & \\
\hline & & & & & & & & & \\
\hline & & & & & & & & & \\
\hline & & & & & & & & & \\
\hline & & & & & & & & & \\
\hline & & & & & & & & & \\
\hline & & & & & & & & & \\
\hline & & & & & & & & & \\
\hline & & & & & & & & & \\
\hline
\end{tabular}

Page ___ of 


\begin{tabular}{|l|c|ll|}
\hline Technical Procedure & $\begin{array}{c}\text { TMI-2 CANISTER DRYING } \\
\text { TESTING AND MONITORING - } \\
\text { ASSEMBLY/DISASSEMBLY AND }\end{array}$ & $\begin{array}{l}\text { Identifier: } \\
\text { Revision: }\end{array}$ & $\begin{array}{l}\text { TPR-1187 } \\
\text { Page: }\end{array}$ \\
TAN Operations & F1 of F2 \\
\hline
\end{tabular}

\section{APPENDIX F \\ DWS Holding Tank Sampling and Pump Out}

NOTE: If uncertain about the TK-1 contents, it is necessary to obtain a sample and analyze it to determine the pump-out route. Water from the DWS that is planned for return to the TAN storage pool must meet the following criteria:

\begin{tabular}{|l|l|}
\hline Conductivity & $<120 \mu \mathrm{mhos} / \mathrm{cm}^{l}$ \\
\hline$p H$ & $6.0-8.5$ \\
\hline Specific Activity & $<0.001 \mu \mathrm{Ci} / \mathrm{ml}^{2}$ \\
\hline Chloride & $<10 \mathrm{ppm}$ \\
\hline Fluorides & $<1 \mathrm{ppm}$ \\
\hline Suspended Solids & $<100 \mathrm{ppm}$ \\
\hline (1) This value exceeds the present pool specification of $<60 \mu \mathrm{mhos} / \mathrm{cm}$ but is considered acceptable for \\
the TAN pool.
\end{tabular}

\section{Pumping out the DWS Holding Tank (TK-1) Contents}

1. If necessary, determine where TK-1 contents will be discharged based on the TK-1 sample results (Step Circulating and Sampling of the DWS Holding Tank (TK-1) Contents, given below).

2. Connect, or ensure connected, FH-9 to QC-13.

3. Depending on the discharge location complete one of the following for routing FH-9:

a. TAN Pool - route FH-9 to the pool vestibule.

b. Radioactive Waste Tanks - obtain approval and route to a waste collection system.

4. Record LI-1 level _ inch

5. Ensure open or open V-15 and open V-26.

6. Start P-1 (SW2, Pump On).

7. Monitor flow on FI-4.

8. P-1 will trip off when the Lower level sensor activates; record LI-1 level inch.

9. Close V-26.

10. Record the amount of water discharged [should be zero] times 4.25).

\section{Circulating and Sampling of the DWS Holding Tank (TK-1) Contents}

1. Ensure that TK-1 water level is visible in the sight glass LI-1.

2. Open or ensure DWS V-21 is $1 / 4$ turn open to establish a circulation flow back to the TK-1.

3. Shut or ensure shut DWS valves V-15, V-23, V-24 and V-26.

4. Start DWS pump P-1 (SW2, Pump On) and ensure circulation flow on DWS FI-5.

5. WAIT 15 minutes, THEN position a sample bottle at the outlet of V-16 to obtain a sample using V-16.

6. Shut off the DWS pump P-1 (SW1, Pump Off).

7. Have the TK-1 sample analyzed.

8. IF suspended solids and/or specific activity are too high,

THEN complete Step Circulating the DWS Holding Tank (TK-1) Contents Through FLT-1 and IX Module, given below. 


\begin{tabular}{|l|c|ll|}
\hline Technical Procedure & $\begin{array}{r}\text { TMI-2 CANISTER DRYING } \\
\text { TESTING AND MONITORING - } \\
\text { ASSEMBLY/DISASSEMBLY AND }\end{array}$ & $\begin{array}{ll}\text { Revision: } \\
\text { TAN Ope: }\end{array}$ & $\begin{array}{l}\text { TPR-1187 } \\
\text { C2 of F2 }\end{array}$ \\
\hline
\end{tabular}

\section{Circulating the DWS Holding Tank (TK- 1) Contents Through FLT-1 and IX Module}

1. Turn DWS V-10 to the $\boldsymbol{B}$ position.

2. Start DWS pump P-1 (SW2, Pump On).

3. Ensure flow of about one gpm on FI-5. If necessary, adjust V-21 to obtain one gpm flow.

4. Open V-23.

5. Adjust V-15 and/or V-21 to obtain a flow of 2.0 to 2.4 gpm as on FI-4.

6. Continue circulating for the period specified by the Test Engineer (Will depend on the amount of liquid and the amount of solids and activity).

7. Shut of P-1 (SW1, Pump Off),

8. Close V-23.

9. Adjust V-21 to $1 / 4$ turn open.

10. Fully open $\mathrm{V}-15$, if necessary.

11. Turn V-10 from position $\boldsymbol{B}$ to $\boldsymbol{A}$.

12. Resample per Step Circulating and Sampling of the DWS Holding Tank (TK-1) Contents. 


\begin{tabular}{|l|c|ll|}
\hline Technical Procedure & $\begin{array}{c}\text { TMI-2 CANISTER DRYING } \\
\text { TESTING AND MONITORING - } \\
\text { ASSEMBLY/DISASSEMBLY AND }\end{array}$ & $\begin{array}{l}\text { Identifier: } \\
\text { Revision: }\end{array}$ & $\begin{array}{l}\text { TPR-1187 } \\
\text { Page: }\end{array}$ \\
TAN Operations & GHECKOUT & & \\
\hline
\end{tabular}

\section{APPENDIX G DWS Valve Checklist}

Procedure Step

Test Name

\begin{tabular}{|c|c|c|c|}
\hline Valve \# & Function & Normal Position & Initial \\
\hline $\mathrm{V}-1$ & Air Supply Solenoid Isolation & Closed (De-Energized) & \\
\hline $\mathrm{V}-2$ & Demineralized Water Supply & Closed & \\
\hline $\mathrm{V}-3$ & Demineralized Water to TK-1 Spray & Closed & \\
\hline $\mathrm{V}-4$ & Canister Back-Flush & Closed & \\
\hline $\mathrm{V}-5$ & Canister Dewatering & Open & \\
\hline $\mathrm{V}-6$ & Back-Flush PI-2 Gauge Isolation & Open & \\
\hline $\mathrm{V}-7$ & Canister Depressurization & Closed & \\
\hline $\mathrm{V}-8$ & Dewatering PI-3 Gauge Isolation & Open & \\
\hline $\mathrm{V}-9$ & Canister Inlet 3-Way & Position $\boldsymbol{A}$ & \\
\hline $\mathrm{V}-10$ & Canister Outlet 3-way & Position $\boldsymbol{A}$ & \\
\hline $\mathrm{V}-11$ & Canister Outlet PI-4 Gauge Isolation & Open & \\
\hline $\mathrm{V}-12$ & Canister Outlet Sample & Closed & \\
\hline $\mathrm{V}-13$ & Filter FLT-1 Outlet & Open & \\
\hline V-14 & FLT-1 Outlet PI-5 Gauge Isolation & Open & \\
\hline $\mathrm{V}-15$ & Pump P-1 Discharge Throttle & Open (Throttled) & \\
\hline $\mathrm{V}-16$ & Pump P-1 Discharge Sample & Closed & \\
\hline $\mathrm{V}-17$ & Pump P-1 Discharge PI-6 Gauge Isolation & Open & \\
\hline $\mathrm{V}-18$ & Tank TK-1 Drain & Closed & \\
\hline $\mathrm{V}-19$ & Automatic Vent Valve VTV-1 Vent Loop Isolation & Open & \\
\hline $\mathrm{V}-20$ & IX-1 Outlet to Tank & Open & \\
\hline $\mathrm{V}-21$ & Pump Discharge to Tank Recirculation Throttle & Open (Throttled) & \\
\hline $\mathrm{V}-22$ & Pump Suction From Tank & Open & \\
\hline $\mathrm{V}-23$ & Pump/FLT-1/IX-1/TK-1 Recirculation Isolation & Closed & \\
\hline $\mathrm{V}-24$ & Pump/IX-1/TK-1 Recirculation Isolation & Closed & \\
\hline $\mathrm{V}-25$ & QC-15 Interface Line Isolation & Closed & \\
\hline $\mathrm{V}-26$ & Pump Discharge to QC-13 & Closed & \\
\hline $\mathrm{V}-27$ & HEPA Filter Housing Drain & Closed & \\
\hline $\mathrm{V}-28$ & Air Supply Manual Isolation & Closed & \\
\hline $\mathrm{V}-29$ & Liquid Drain Valve Bypass & Closed & \\
\hline $\mathrm{V}-30 \mathrm{~A}$ & Liquid Drain Valve DR-1A Isolation & Open & \\
\hline $\mathrm{V}-30 \mathrm{~B}$ & Liquid Drain Valve DR-1B Isolation & Open & \\
\hline $\mathrm{V}-30 \mathrm{C}$ & Liquid Drain Valve DR-1C Isolation & Open & \\
\hline V-30D & Liquid Drain Valve DR-1D Isolation & Open & \\
\hline $\mathrm{V}-31$ & Auto Vent Valve VTV-1 Vent Loop Isolation & Open & \\
\hline V-32 & VTV-2 Liquid Drain Valve DR-2 Isolation & Open & \\
\hline V-33 & VTV-2 Outlet Back pressure Throttle Valve & Open (Throttled-Do not close) & \\
\hline $\mathrm{V}-34$ & HVDS Condensate Interface Isolation & Closed & \\
\hline
\end{tabular}

Test Number 
Technical Procedure

TAN Operations
TMI-2 CANISTER DRYING TESTING AND MONITORING ASSEMBLY/DISASSEMBLY AND CHECKOUT
Identifier: TPR-1187

Revision: 1

Page: $\quad$ H1 of $\mathrm{H} 1$

\section{APPENDIX H}

Test Personnel Identification

\begin{tabular}{|l|l|l|l|}
\hline \multicolumn{2}{|c|}{ Name } & Initials & \multicolumn{1}{|}{ Assignment } \\
\hline & & & Test Engineer \\
\hline & & & \\
\hline & & & \\
\hline & & & Quality Inspector \\
\hline & & & \\
\hline & & & \\
\hline & & & Technician \\
\hline & & & \\
\hline & & & \\
\hline & & & \\
\hline & & & \\
\hline & & & \\
\hline & & & \\
\hline & & & Others \\
\hline & & & \\
\hline & & & \\
\hline & & & \\
\hline & & & \\
\hline
\end{tabular}




\begin{tabular}{|l|c|ll|}
\hline Technical Procedure & $\begin{array}{c}\text { TMI-2 CANISTER DRYING } \\
\text { TESTING AND MONITORING - } \\
\text { TAN Operations }\end{array}$ & $\begin{array}{l}\text { Identifier: } \\
\text { Revision: }\end{array}$ & $\begin{array}{l}\text { TPR-1187 } \\
\text { ASSEMBLY/DISASSEMBLY AND } \\
\text { Page: }\end{array}$ \\
\hline
\end{tabular}

\section{APPENDIX I \\ Definitions}

\section{Acceptance Criteria - A canister is defined to be dry at a moderator density $\left(\mathrm{H}_{2} \mathrm{O}\right)$ of $8.8 \times 10^{-5} \mathrm{~g} / \mathrm{cc}$ or less.}

Acceptance Test - Testing which allows for the inference that a canister is dry. For this procedure, the acceptance test for a canister to be inferred dry is the maintenance of $<80$ torr vacuum for 30 minutes at $120^{\circ} \mathrm{F}$.

Dewatering Skid - The equipment that removes water from the TMI-2 canisters; after removal the water is passed through a filter and ion exchanger to remove suspended solids and radioactivity.

Lower Explosive Limit (LEL) - The boundary mixture (minimum concentration of the material) of a gas (hydrogen) which, if ignited, will just propagate a flame. The lower explosive limit of hydrogen at atmospheric temperature and pressure is 4 percent by volume in air. The terms "flammable limits" and "explosive limits" are interchangeable.

Radiolysis - Chemical decomposition by the action of radiation; for these tests this is determined by the measurement of hydrogen evolution from the decomposition of residual water in a canister due to the radioactive energy emitted by the contents of a canister.

Vacuum Condenser Skid - Refrigeration equipment for removing water vapor from the TMI-2 canisters off gas from the Vacuum Furnace.

Vacuum Furnace - A vessel capable of heating one to four TMI-2 canisters in a vacuum environment.

Vacuum Pump Skid - Equipment capable of developing a vacuum on (or pressurizing) a closed system (Vacuum Furnace, shielding Cask, or Dry Shielded Canister. 


\begin{tabular}{|l|c|ll|}
\hline Technical Procedure & $\begin{array}{c}\text { TMI-2 CANISTER DRYING } \\
\text { TESTING AND MONITORING - } \\
\text { TAN Operations }\end{array}$ & $\begin{array}{ll}\text { Identifier: } \\
\text { Revision: } \\
\text { ASSEMBLY/DISASSEMBLY AND }\end{array}$ & $\begin{array}{l}\text { TPR-1187 } \\
\text { Page: }\end{array}$ \\
\hline
\end{tabular}

APPENDIX J

Procedure Basis

\begin{tabular}{|c|c|c|}
\hline Step Number & Step Basis & Information Source \\
\hline Entire procedure & Develop test procedures. & $\begin{array}{l}\text { PRD-101, Section 10.3.1.3 } \\
\text { PRD-101, Section 10.3.2.1 } \\
\text { PRD-101, Section 10.3.2.3 }\end{array}$ \\
\hline Entire procedure & $\begin{array}{l}\text { Test requirements and acceptance } \\
\text { criteria. }\end{array}$ & PRD-101, Section 10.3.1.2 \\
\hline
\end{tabular}




Lockheed Martin Idaho Technologies Company
\begin{tabular}{|l|r|ll|}
\hline Technical Procedure & TMI-2 CANISTER DRYING \\
TESTING AND MONITORING - & $\begin{array}{l}\text { Identifier: } \\
\text { Revision: } \\
\text { Page: }\end{array}$ & $\begin{array}{l}\text { TPR-12.09\# } \\
10 / 20 / 97-\text { Rev. } \# 01)\end{array}$ \\
TAN Operations & FUNCTIONAL TESTING 11 \\
\hline $\begin{array}{l}\text { Document Control Center: } \\
\text { (208) 526-6076 }\end{array}$ & Document Owner/Approver: TANO Supervisor & Effective Date: & canceled \\
\hline Manual: DOP & CATEGORY 3 & DAR Number: & $\underline{\text { TANO-5452 }}$ \\
\hline
\end{tabular}

Validation - This document must be validated prior to first use by one of the following:

[ ] Walkthrough

[ ] Using mockup or simulators

Signature
[ ] Tabletop validation by safety committee and craftsmen [ ] First time use of procedure
Date 


\begin{tabular}{|l|c|ll|}
\hline Technical Procedure & TMI-2 CANISTER DRYING & Identifier: & TPR-1188 \\
& TESTING AND MONITORING - & Revision: & 0 \\
Tage: & $\mathbf{2}$ of 11 \\
\hline
\end{tabular}

\section{REVISION LOG}

\begin{tabular}{|c|c|c|c|}
\hline Rev. & Date & $\begin{array}{c}\text { Affected } \\
\text { Pages }\end{array}$ & Revision Description \\
\hline 0 & 03/10/98 & All & Initial release. See DAR TANO-5452. \\
\hline
\end{tabular}




\begin{tabular}{|l|c|ll|}
\hline Technical Procedure & TMI-2 CANISTER DRYING & Identifier: & TPR-1188 \\
& TESTING AND MONITORING - & Revision: & 0 \\
Page: & $\mathbf{3}$ of 11 \\
\hline
\end{tabular}

\section{INTRODUCTION}

\subsection{Purpose}

Definition of the systematic actions required for performing Functional Testing of the heated vacuum drying system (HVDS) will ensure satisfactory completion of the testing program.

\subsection{Scope and Applicability}

Functional testing is performed to determine drying temperatures and times for various Vacuum Furnace operations and configurations (type and/or number of canisters). These tests are performed on "Cold" canisters filled with demineralized water plus simulated debris and then dewatered to assess the drying process. The HVDS consists of the Vacuum Pump Skid (VPS), the Vacuum Condenser Skid (VCS), and the Vacuum Furnace (VF) (see Definitions).

This procedure applies only to those persons performing the HVDS TMI-2 Canister Drying Testing And Monitoring - Functional Testing.

\section{PRECAUTIONS AND LIMITATIONS}

2.3 All high temperature test equipment exposed to personnel during operations is clearly marked and proper precautions are taken when the equipment is approached.

2.4 Personnel restrictions apply in the TAN Warm Shop while a TMI canister is being handled.

\section{PREREQUISITES}

\subsection{Planning and Coordination}

3.1.1 Hoisting and rigging equipment load test is current.

3.1.2 Equipment operators are qualified and current.

3.1.4 Personnel performing this procedure are trained.

3.1.5 The HVDS has been assembled in the Warm Shop (Procedure TPR-1187).

3.1.6 HVDS checkout (Procedure TPR-1187) has been successfully completed in the TAN Warm Shop. 


\begin{tabular}{|l|c|ll|}
\hline Technical Procedure & TMI-2 CANISTER DRYING & $\begin{array}{l}\text { Identifier: } \\
\text { Revision: }\end{array}$ & $\begin{array}{l}\text { TPR-1188 } \\
\text { TESTING AND MONITORING - } \\
\text { TAN Operations }\end{array}$ \\
\hline
\end{tabular}

\subsubsection{Instruments calibrated in Procedure TPR-1187 are still in calibration.}

\subsection{Performance Documents}

None

\subsection{Special Tools, Equipment, Parts, and Supplies}

The equipment and materials given in Table 3.3 are available.

Table 3.3 Equipment List

\begin{tabular}{|l|c|l|}
\hline Item & No. & Comments \\
\hline $\begin{array}{l}\text { "Cold" Fuel and Knockout } \\
\text { Canisters }\end{array}$ & 2 each & $\begin{array}{l}\text { Contains measured and recorded amounts of simulated debris and } \\
\text { demineralized water that have been allowed to sit for a minimum of 24 hours } \\
\text { prior to testing }\end{array}$ \\
\hline Nitrogen Supply & 1 & To be used to pressurize the system (minimum of 500 $\mathrm{ft}^{3}$ ) \\
\hline $\begin{array}{l}\text { TMI Canister short } \\
\text { grapple assembly }\end{array}$ & 1 & $\begin{array}{l}\text { Calibration Verification Expiration Date } \\
\text { Quality Inspector } \\
\text { Date }\end{array}$ \\
\hline Slings and shackles & 1 & Rigging for TMI grapples \\
\hline $\begin{array}{l}\text { TMI storage module } \\
\text { vertical lift rigging }\end{array}$ & $\begin{array}{l}\text { Calibration Verification Expiration Date } \\
\text { Quality Inspector } \\
\text { Date }\end{array}$ \\
\hline $\begin{array}{l}\text { Dynamometer to 5,000 lb. } \\
\text { S/N }\end{array}$ & $\begin{array}{l}\text { Calibration Verification Expiration Date } \\
\text { Quality Inspector } \\
\text { Date }\end{array}$ \\
\hline
\end{tabular}

\subsection{Preparations}

Test Engineer: Conduct a briefing with the test personnel and complete the following items:
A. A discussion of safety precautions and emergency actions associated with the operation of the HVDS.
B. A review of Section 4 of this test procedure.
C. The table, Test Personnel Identification, in Appendix F.

Test Engineer

Date

\subsection{Approval and Notifications}

Review this procedure. Sign space indicated and enter date for approval of work to commence. 


\begin{tabular}{|l|c|ll}
\hline Technical Procedure & TMI-2 CANISTER DRYING & Identifier: & TPR-1188 \\
& TESTING AND MONITORING - & Revision: & 0 \\
TAn Operations & FUNCTIONAL TESTING & $\mathbf{5}$ of 11 \\
\hline
\end{tabular}

TANO Shift Supervisor: Date

Operations Technician Date

\section{INSTRUCTIONS}

\subsection{Test Instructions}

\subsubsection{The Test Engineer:}

A. Verifies the test operation by collecting data and signing where called for.

B. Signs and dates the completion of test sub-sections; checks or signatures do not imply complete data evaluation.

C. May collect data generated for test sections.

D. Maintains the master copy of the procedure and logs for report generation.

E. May alter the sequence in which the test is conducted if needed.

F. Makes test procedure changes as needed.

G. Designates an alternate to act for him/her if desired.

H. Establishes authority by identifying the responsibility and initials of the test participants (Appendix F).

\subsubsection{The Quality Inspector:}

A. Verifies the data and performance of the test.

B. Verifies test procedure changes made by the Test Engineer.

C. Signs and dates the completion of test sub-sections.

\subsubsection{The Technician:}

A. Verifies the test operation by filling the blanks in the tables and by checking off at spaces provided at the left of procedure steps.

B. Prepares copies of appendixes as needed for test sections.

C. Collects data generated for test sections.

4.1.4 All test personnel check other non-specified items during the test, including but not limited to the labeling of piping, electrical conduits, and instruments and components.

\subsubsection{Test Description}

The HVDS is designed to be fully automatic using programmable control logic (PCL) technology. The sequence of operation is as follows: 


\begin{tabular}{|l|c|ll|}
\hline Technical Procedure & TMI-2 CANISTER DRYING & Identifier: & TPR-1188 \\
& TESTING AND MONITORING - & Revision: & 0 \\
Page: & $\mathbf{6}$ of 11 \\
\hline
\end{tabular}

1) The remote Start button is pushed.

2) V7 is opened.

3) Furnace heaters are controlled to $180^{\circ} \mathrm{F}$.

4) V1 is opened when a pump temperature of $150^{\circ} \mathrm{F}$ is reached.

5) Furnace heaters are allowed to increase when a vacuum pressure of 80 torr is reached.

6) A working temperature of $900^{\circ} \mathrm{F}$ is maintained using pressure compensated control heaters.

7) Completion is signaled when a vacuum pressure of $<1$ torr is achieved at $900^{\circ} \mathrm{F}$.

8) The heaters are shut off and the vacuum furnace is allowed to cool until the temperature is stabilized (about two hours with a furnace temperature of 700$800^{\circ} \mathrm{F}$ or the temperature change is $<20^{\circ} \mathrm{F} /$ hour).

9) V1 is closed.

10) If vacuum pressure is not maintained at $<3$ torr for 30 minutes, $V 1$ is opened and after stable temperature, the test is repeated; this is repeated two times, if necessary, before the process continues.

11) The system is allowed to cool to $120^{\circ} \mathrm{F}$.

12) Acceptance testing is performed $\left(120^{\circ} \mathrm{F}\right.$ and $<80$ torr for 30 minutes).

13) The system is shutdown whether or not acceptance criteria were met.

14) The design includes logic for performing manual acceptance testing.

This test consists of the following sections: Pretest Preparation, Vacuum Furnace Canister Loading, Canister Drying Operation, and Vacuum Furnace Canister Unloading. The test engineer determines the type of canisters to be used, combination, and number of drying operations.

Functional Testing is performed in the TAN Warm Shop using "Cold" canisters to demonstrate HVDS operation. Additionally, the testing provides a mechanism for hands-on operator training prior to testing "Hot" canisters. 


\begin{tabular}{|l|c|ll|}
\hline Technical Procedure & TMI-2 CANISTER DRYING & Identifier: & TPR-1188 \\
& TESTING AND MONITORING - & Revision: & 0 \\
Tage: & 7 of 11 \\
\hline
\end{tabular}

\subsection{Pretest Preparation}

4.1.17 Tech: Align switches (Appendix C Switch Checklist) and valves (Appendix B Valve Checklist) for Normal (Automatic) Operation.

4.1.18 Close isolation valves, V1 and V7, (switches in the $\boldsymbol{O} f \boldsymbol{f}$ position) for vacuum pump warm-up.

4.1.19 Start the vacuum pump (Manual button on the VPS) for warm-up (1-hour).

4.1.20 Visually check the level of DWS Holding Tank; IF the level is not near the bottom of the sight glass, THEN use the procedure given in Appendix E, DWS Holding Tank Sampling and Pump Out, to transfer the tank contents.

4.1.21 Connect a regulated nitrogen supply (at least $500 \mathrm{ft}^{3}$ ) to the V4 fitting location (V4 should be closed).

\subsection{Vacuum Furnace Canister Loading}

4.3.1 Tech: Prepare to open the VF head by pressing the remote Open/Close button.

4.3.2 EO: Lift the head until it rests against the hinge stops using the Warm Shop crane lift.

4.3.3 Attach the Warm Shop bridge crane hook to the 5,000-lb. dynamometer and the TMI short grapple.

NOTE: The maximum allowable weight of the loaded canister during handling operations is 2,940 lb.

4.3.4 Lift the TMI short grapple out of its support stand.

4.3.5 Tech: Record the grapple weight lbs.

4.3.6 Attach the TMI short grapple to a test canister to be loaded.

4.3.7 EO: Use an observer during lifting, lowering or moving the canister in the following steps to ensure all obstacles are cleared.

4.3.8 Carefully lift the canister while the technician accomplishes the following step.

4.3.9 Tech: Obtain the canister weight as follows: 


\begin{tabular}{|l|c|ll|}
\hline Technical Procedure & TMI-2 CANISTER DRYING & Identifier: & TPR-1188 \\
& TESTING AND MONITORING - & Revision: & 0 \\
Tage: & $\mathbf{8}$ of 11 \\
\hline
\end{tabular}
A. Observe the weight of canister as it is lifted.
B. IF weight exceeds $2,940 \mathrm{lb}$, THEN STOP, lower and secure canister, $\underline{\mathrm{AND}}$ correct the problem before proceeding.
C. Record the combined weight of canister and grapple assembly
D. Record the canister weight (combined weight minus grapple weight) lbs. lbs.

4.3.10 EO: After the canister is about 1-foot from the floor, move it near the VF prior to continuing the lift.

4.3.11 Lift the canister high enough to clear the top of the VF.

4.3.12 Move the canister over one of the receiving positions.

4.3.13 Carefully lower the canister into the VF.

4.3.14 Tech: Visually ensure that the canister is seated correctly into the VF (the canister should be less than 4 -inches above the top alignment-plate).

4.3.15 After the canister is properly seated, detach the TMI short grapple.

4.3.16 Repeat steps 4.3.4 through 4.3.15 until the desired number of canisters (up to four) have been loaded.

4.3.17 Remove the 1/4- and 3/8-inch Hansen couplings from each of the canisters (fittings must be saved for reinstallation).

4.3.18 EO: Lower the VF head to its closed position using the Warm Shop crane lift.

4.3.19 Tech: Complete the closure of the VF head by pressing the remote Open/Close button.

\subsection{Canister Drying Operation}

4.4.7 Tech: Align the HVDS switches for Normal Operation (Appendix C).

4.4.8 Press the remote Start button to begin automatic operation.

4.4.9 Record temperature and pressure data in Test Log (Appendix D) at 15-minute increments UNTIL the system shuts down and the PLC temperature is below $120^{\circ} \mathrm{F}$. 


\begin{tabular}{|l|c|ll|}
\hline Technical Procedure & TMI-2 CANISTER DRYING & Identifier: & TPR-1188 \\
& TESTING AND MONITORING - & Revision: & 0 \\
Page: & 9 of 11 \\
\hline
\end{tabular}

\subsubsection{Acceptance Criteria}

When the remote Start button is pressed, the HVDS automatically begins the vacuum and heating process, senses drying completion, performs the Acceptance Test ( $<80$ torr for 30 minutes) and shuts down the system.

4.4.11 The operation performed as expected and met the acceptance criteria.

Test Engineer Date

Quality Inspector Date

\subsection{Vacuum Furnace Canister Unloading}

4.5.1 Tech: Align valves for Furnace Pressurization (Appendix B).

Note: For V1 to be open, its switch is in the Manual position. For V7 to be closed, its switch is in the Off position.

4.5.2 Adjust V4 to slowly purge $\mathrm{N}_{2}$ to the system UNTIL PI1 reads about +2 psig, THEN close V4.

4.5.3 Obtain particulate sample (for training and checkout only).
A. Attach the sample apparatus to the sampling connection at V11.
B. Close V1 ( Off $)$.
C. Open V11 to obtain the sample, THEN close V11.
D. Open V1 (Manual).

4.5.4 Open V10 until the pressure at PI1 returns to atmospheric, THEN close V10.

4.5.5 Start the head sweep system.

4.5.6 Prepare to open the VF head by pressing the remote $\boldsymbol{O p e n / C l o s e}$ button.

4.5.7 EO: Lift the head until it rests against the hinge stops using the Warm Shop crane lift.

4.5.8 Tech: Stop the head sweep system.

4.5.9 Reinstall the 1/4- and 3/8-inch Hansen couplings in all the canisters. 


\begin{tabular}{|l|c|ll|}
\hline Technical Procedure & TMI-2 CANISTER DRYING & Identifier: & TPR-1188 \\
& TESTING AND MONITORING - & Revision: & 0 \\
Tane: & $\mathbf{1 0}$ of 11 \\
\hline
\end{tabular}

4.5.10 EO: Attach the Warm Shop bridge crane hook to the 5,000-lb. dynamometer and the TMI short grapple.

4.5.11 Lift the TMI short grapple out of its support stand.

4.5.12 Tech: Record the grapple weight lbs.

4.5.13 EO: Use an observer during lifting, lowering or moving the canister in the following steps to ensure all obstacles are cleared.

4.5.14 Move the grapple to a test canister to be removed and make the attachment.

4.5.15 Carefully lift the canister while the technician accomplishes the following step.

4.5.16 Tech: Obtain the canister weight as follows:

A. Observe the weight of canister as it is lifted.

B. IF weight exceeds 2,940 lb., THEN STOP, lower and secure canister, $\underline{\text { AND }}$ correct the problem before proceeding.

C. Record the combined weight of canister and grapple assembly lbs.

D. Record the canister weight (combined weight minus grapple weight) lbs.

4.5.17 EO: Lift the canister high enough to clear the top of the VF.

4.5.18 Lower the canister to about 1-foot from the floor as soon as it has cleared the VF.

4.5.19 Move the canister to a storage position (lift as necessary to place the canister in the storage position).

4.5.20 Tech: After the canister is properly secured, detach the TMI short grapple.

4.5.21 Repeat steps 4.5.9 through 4.5.15 until all the canisters have been removed.

4.5.22 EO: Lower the VF head to its closed position using the Warm Shop crane lift.

4.5.23 Tech: Complete the closure of the VF head by pressing the remote Open/Close button.

4.5.24 Realign valves (Appendix B) and switches (Appendix C) for Normal Operation. 


\begin{tabular}{|l|c|ll|}
\hline Technical Procedure & TMI-2 CANISTER DRYING & Identifier: & TPR-1188 \\
& TESTING AND MONITORING - & Revision: & 0 \\
Page: & $\mathbf{1 1}$ of 11 \\
\hline
\end{tabular}

\subsection{Additional Canister Drying (if called for by the Test Engineer)}

4.6.1 Tech: Use the procedure given in Appendix E, DWS Holding Tank Sampling and Pump Out, to transfer the tank contents.

4.6.2 If bottled $\mathrm{N}_{2}$ is used, check to verify the $\mathrm{N}_{2}$ supply is adequate $\left(500 \mathrm{ft}^{3}\right)$.

4.6.3 Repeat Sections 4.3 through 4.5 for each additional drying test.

\section{POST PERFORMANCE ACTIVITIES}

None

6. RECORDS

NOTE: $\quad$ Records shall be kept in the project file.

\begin{tabular}{|c|l|l|}
\hline \multicolumn{1}{|c|}{ Record } & Retention & \multicolumn{1}{c|}{ Disposition } \\
\hline This procedure, including all data sheets when completed & Permanent & Destroy when no longer needed \\
\hline
\end{tabular}

\section{SOURCE REQUIREMENTS}

None

\section{APPENDICES}

Appendix A, Figure 1

Appendix B, HVDS Valve Checklist

Appendix C, Switch Checklist

Appendix D, Drying Test Log

Appendix E, DWS Sampling and Pump Out

Appendix F, Test Personnel Identification

Appendix G, Definitions 


\begin{tabular}{|l|c|ll|}
\hline Technical Procedure & TMI-2 CANISTER DRYING & Identifier: & TPR-1188 \\
& TESTING AND MONITORING - & Revision: & 0 \\
TAN Operations & FUNCTIONAL TESTING & Page: & A1 of A1 \\
\hline
\end{tabular}

\section{APPENDIX A}

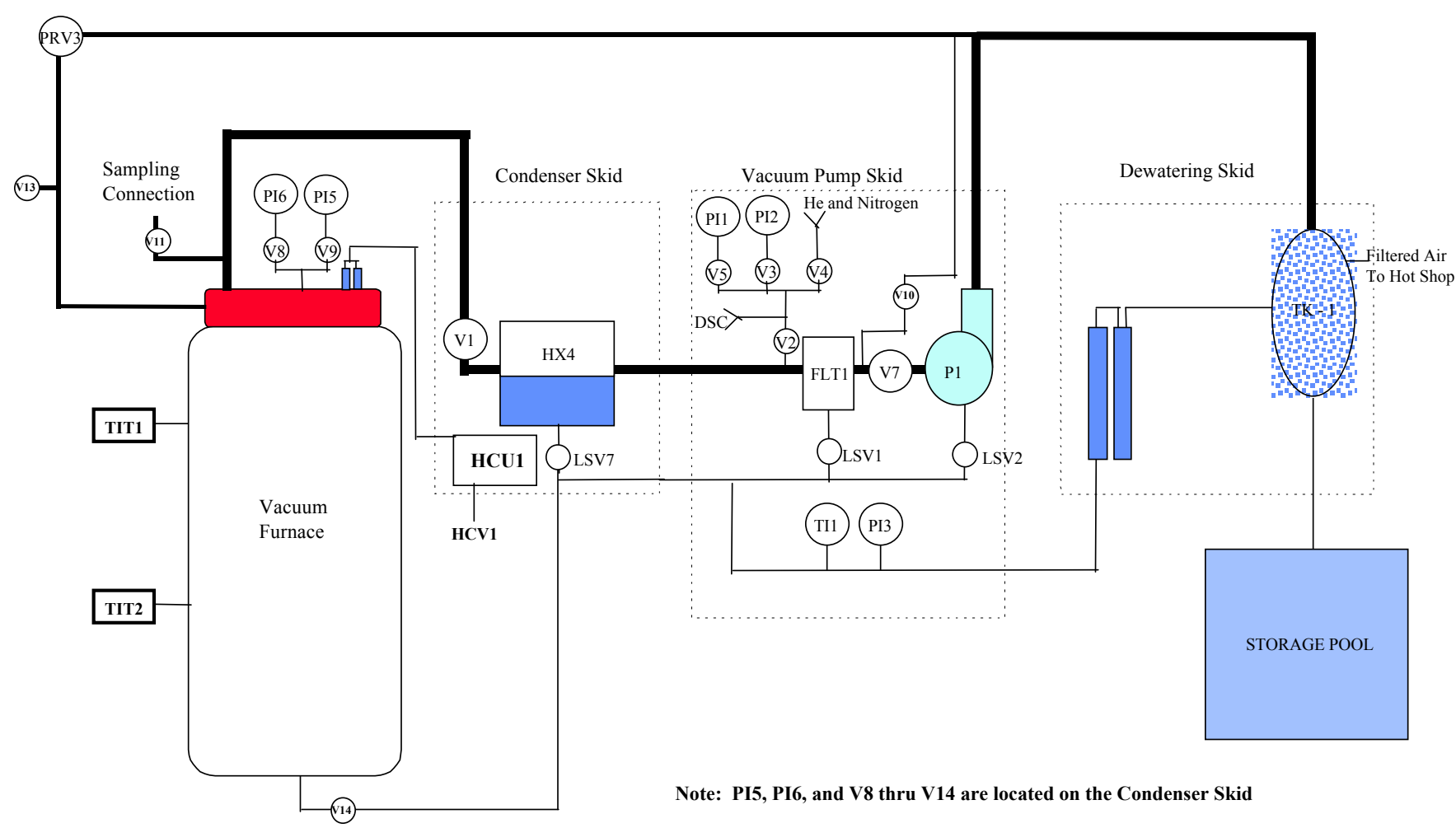

Figure 1 Heated Vacuum Drying System (HVDS) Schematic. 


\begin{tabular}{|l|c|ll|}
\hline Technical Procedure & TMI-2 CANISTER DRYING & Identifier: & TPR-1188 \\
& TESTING AND MONITORING - & Revision: & 0 \\
Tage: & B1 of B1 \\
\hline
\end{tabular}

\section{APPENDIX B}

\section{HVDS Valve Checklist}

Test Name

Test Dates

Test Number

\begin{tabular}{|c|c|c|c|c|c|}
\hline $\begin{array}{l}\text { Valve } \\
\text { Number } \\
\end{array}$ & $\begin{array}{l}\text { Normal } \\
\text { Operation }\end{array}$ & $\begin{array}{l}\text { Acceptance } \\
\text { Testing }\end{array}$ & $\begin{array}{l}\text { Furnace } \\
\text { Pressurization }\end{array}$ & Vacuum Furnace Sampling & Initials \\
\hline V1 & Opened & Closed & Opened & Closed & \\
\hline V2 & Opened & Opened & Opened & Opened & \\
\hline V3 & Closed & Closed & Closed & Closed & \\
\hline V4 & Closed & Closed & Closed & Closed & \\
\hline V5 & Opened & Opened & Opened & Opened & \\
\hline V7 & Opened & Closed & Closed & Closed & \\
\hline $\mathrm{V} 8$ & Opened & Opened & Closed & Closed & \\
\hline V9 & Opened & Opened & Closed & Closed & \\
\hline V10 & Closed & Closed & Closed & Closed & \\
\hline V11 & Closed & Closed & Closed & Opened & \\
\hline V14 & Closed & Closed & Closed & May be used to purge furnace & \\
\hline V15 & Closed & Closed & Closed & Closed & \\
\hline V16 & Closed & Closed & Closed & Normally Closed (Handle Removed) & \\
\hline V17 & & & & Preset (Handle Removed) & \\
\hline V18 & Closed & Closed & Closed & Closed & \\
\hline \multicolumn{6}{|c|}{$\begin{array}{l}\text { Notes: } \\
\text { 4. Valves are identified in Appendix A Figure } 1 . \\
\text { 5. Ensure that the Air Sampler is installed prior to opening valve V11 when performing "Canister Testing" } \\
6 . \quad \text { Valve indication lights are illuminated when valves are opened. }\end{array}$} \\
\hline
\end{tabular}




\begin{tabular}{|l|c|ll|}
\hline Technical Procedure & TMI-2 CANISTER DRYING & Identifier: & TPR-1188 \\
& TESTING AND MONITORING - & Revision: & 0 \\
Tane: & C1 of C1 \\
\hline
\end{tabular}

APPENDIX C

Switch Checklist

Test Name

Test Dates

Test Number

\begin{tabular}{|l|l|l|l|l|l|l|}
\hline \multicolumn{1}{|c|}{$\begin{array}{l}\text { Switch } \\
\text { Name }\end{array}$} & Location & Label & $\begin{array}{l}\text { Normal } \\
\text { Operation } \\
\text { (Automatic) }\end{array}$ & $\begin{array}{l}\text { Manual } \\
\text { Operation }\end{array}$ & $\begin{array}{l}\text { Off } \\
\text { Position }\end{array}$ & Initial \\
\hline $\begin{array}{l}\text { Coolant Circulation } \\
\text { Motor }\end{array}$ & VCS & Coolant Pum0 & On & On & Off & \\
\hline $\begin{array}{l}\text { Refrigeration } \\
\text { System Motor }\end{array}$ & VCS & Refr System & Auto & Manual & Off & \\
\hline Valve V1 Operator & VCS & V1 & Auto & Manual & Off & \\
\hline & & & & & & \\
\hline Heater 1 & VF & Heat Bank 1 & Auto & Off & NA $^{*}$ & \\
\hline Heater 2 & VF & Heat Bank 2 & Auto & Off & NA $^{*}$ & \\
\hline $\begin{array}{l}\text { Hydraulic Closer } \\
\text { Operation }\end{array}$ & VF & & $\begin{array}{l}\text { Open or Closed } \\
\text { as appropriate }\end{array}$ & $\begin{array}{l}\text { Open or Closed } \\
\text { as appropriate }\end{array}$ & Off & \\
\hline & & & & & & \\
\hline Oil Pump & VPS & Oil Pump & Auto & Manual & Off & \\
\hline Vacuum Pump & VPS & Vacuum Pump & Auto & Manual & Off & \\
\hline Valve V7 Operator & VPS & V7 & Auto & Manual & Off & \\
\hline
\end{tabular}

${ }^{*} \mathrm{NA}=$ Not Applicable 
Technical Procedure

TAN Operations
TMI-2 CANISTER DRYING TESTING AND MONITORING FUNCTIONAL TESTING
Identifier:

Revision

Page:
TPR-1188

0

D1 of D1

APPENDIX D

\section{Drying Test Log}

Test Name

Test Dates

Test Number

\begin{tabular}{|c|c|c|c|c|c|c|c|c|}
\hline $\begin{array}{l}\text { TIME } \\
\text { (min) }\end{array}$ & $\begin{array}{c}\text { PLC } \\
\text { TEMP }\left({ }^{\circ} \mathbf{F}\right)\end{array}$ & $\begin{array}{l}\text { PI1 } \\
\text { (psi) }\end{array}$ & $\begin{array}{l}\text { PI2 } \\
\text { (torr) }\end{array}$ & $\begin{array}{l}\text { PI3 } \\
\text { (psi) }\end{array}$ & $\begin{array}{l}\text { PI5 } \\
\text { (torr) }\end{array}$ & $\begin{array}{c}\text { PI6 } \\
\text { (torr) }\end{array}$ & INITIAL & COMMENTS \\
\hline & & & & & & & & \\
\hline & & & & & & & & \\
\hline & & & & & & & & \\
\hline & & & & & & & & \\
\hline & & & & & & & & \\
\hline & & & & & & & & \\
\hline & & & & & & & & \\
\hline & & & & & & & & \\
\hline & & & & & & & & \\
\hline & & & & & & & & \\
\hline & & & & & & & & \\
\hline & & & & & & & & \\
\hline & & & & & & & & \\
\hline & & & & & & & & \\
\hline & & & & & & & & \\
\hline & & & & & & & & \\
\hline & & & & & & & & \\
\hline & & & & & & & & \\
\hline & & & & & & & & \\
\hline & & & & & & & & \\
\hline & & & & & & & & \\
\hline & & & & & & & & \\
\hline & & & & & & & & \\
\hline & & & & & & & & \\
\hline & & & & & & & & \\
\hline & & & & & & & & \\
\hline
\end{tabular}




\begin{tabular}{|l|c|ll|}
\hline Technical Procedure & TMI-2 CANISTER DRYING & Identifier: & TPR-1188 \\
& TESTING AND MONITORING - & Revision: & 0 \\
Tage: & E1 of E2 \\
\hline
\end{tabular}

\section{APPENDIX E}

\section{DWS Holding Tank Sampling and Pump Out}

Note: If uncertain about the TK-1 contents, it is necessary to obtain a sample and analyze it to determine the pump-out route. Water from the DWS that is planned for return to the TAN storage pool must meet the following criteria:

\begin{tabular}{|l|l|}
\hline Conductivity & $<120 \mu \mathrm{mhos} / \mathrm{cm}^{l}$ \\
\hline$p H$ & $6.0-8.5$ \\
\hline Specific Activity & $<0.001 \mu \mathrm{Ci} / \mathrm{ml}^{2}$ \\
\hline Chloride & $<10 \mathrm{ppm}$ \\
\hline Fluorides & $<1 \mathrm{ppm}$ \\
\hline Suspended Solids & $<100 \mathrm{ppm}$ \\
\hline $\begin{array}{l}\text { (1) This value exceeds the present pool specification of }<60 \mu \mathrm{mhos} / \mathrm{cm} \text { but is considered acceptable for } \\
\text { the TAN pool. }\end{array}$ \\
\hline (2) This value does not include the activity due to Tritium \\
\hline
\end{tabular}

\section{Pumping out the DWS Holding Tank (TK-1) Contents}

1. If necessary, determine where TK-1 contents will be discharged based on the TK-1 sample results (Step Circulating and Sampling of the DWS Holding Tank (TK-1) Contents, given below).

2. Connect, or verify connected, FH-9 to QC-13.

3. Depending on the discharge location complete one of the following for routing FH-9:

a. TAN Pool - route FH-9 to the pool vestibule.

b. $\quad$ Radioactive Waste Tanks - obtain approval and route to a waste collection system.

4. Record LI-1 level __ inch

5. Verify open or open V-15 and open V-26.

6. Start P-1 (SW2, Pump On).

7. Monitor flow on FI-4.

8. P-1 will trip off when the Lower level sensor activates; record LI-1 level ___ inch.

9. Close V-26.

10. Record the amount of water discharged Step 6 [should be zero] times 4.25).

\section{Circulating and Sampling of the DWS Holding Tank (TK-1) Contents}

1. Verify that TK-1 water level is visible in the sight glass LI-1.

2. Open or verify DWS V-21 is $1 / 4$ turn open to establish a circulation flow back to the TK-1.

3. Shut or verify shut DWS valves V-15, V-23, V-24 and V-26.

4. Start the DWS pump P-1 (SW2, Pump On) and verify circulation flow on DWS FI-5.

5. WAIT 15 minutes, THEN position a sample bottle at the outlet of V-16 to obtain a sample using V-16. 


\begin{tabular}{|l|c|ll|}
\hline Technical Procedure & TMI-2 CANISTER DRYING & Identifier: & TPR-1188 \\
Revision: & 0 \\
TAN Operations & FUNCTIONAL TESTING & Page: & E2 of E2 \\
\hline
\end{tabular}

6. Shut off the DWS pump P-1 (SW1, Pump Off).

7. Have the TK-1 sample analyzed.

8. IF suspended solids and/or specific activity are too high, THEN complete Step Circulating the DWS Holding Tank (TK-1) Contents Through FLT-1 and IX Module, given below.

\section{Circulating the DWS Holding Tank (TK- 1) Contents Through FLT-1 and IX Module}

1. Turn DWS V-10 to the $\boldsymbol{B}$ position.

2. Start DWS pump P-1 (SW2, Pump On).

3. Verify flow of about 1-gpm on FI-5. If necessary, adjust V-21 to obtain 1-gpm flow.

4. Open V-23.

5. Adjust V-15 and/or V-21 to obtain a flow of 2.0 to 2.4 gpm as on FI-4.

6. Continue circulating for the period specified by the Test Engineer (Will depend on the amount of liquid and the amount of solids and activity).

7. Shut of P-1 (SW1, Pump Off),

8. Close V-23.

9. Adjust V-21 to $1 / 4$ turn open.

10. Fully open $\mathrm{V}-15$, if necessary.

11. Turn V-10 from position $\boldsymbol{B}$ to $\boldsymbol{A}$.

12. Resample per Step Circulating and Sampling of the DWS Holding Tank (TK-1) Contents. 
Technical Procedure

TAN Operations
TMI-2 CANISTER DRYING TESTING AND MONITORING FUNCTIONAL TESTING
Identifier:

Revision:

Page:
TPR-1188

0

F1 of F1

APPENDIX F

Test Personnel Identification

\begin{tabular}{|c|c|c|c|}
\hline \multicolumn{3}{|c|}{ Name } & \multirow[b]{2}{*}{ Assignment } \\
\hline Printed & Signature & Initials & \\
\hline & & & Test Engineer \\
\hline & & & \\
\hline & & & \\
\hline & & & Quality Inspector \\
\hline & & & \\
\hline & & & \\
\hline & & & Technician \\
\hline & & & \\
\hline & & & \\
\hline & & & \\
\hline & & & \\
\hline & & & \\
\hline & & & \\
\hline & & & Others \\
\hline & & & \\
\hline & & & \\
\hline & & & \\
\hline & & & \\
\hline & & & \\
\hline & & & \\
\hline
\end{tabular}




\begin{tabular}{|l|c|ll|}
\hline Technical Procedure & TMI-2 CANISTER DRYING & Identifier: & TPR-1188 \\
& TESTING AND MONITORING - & $\begin{array}{l}\text { Revision: } \\
\text { Page: }\end{array}$ & G1 of G1 \\
TAN Operations & FUNCTIONAL TESTING &
\end{tabular}

\section{APPENDIX G}

\section{Definitions}

Acceptance Criteria - A canister is defined to be dry at a moderator density $\left(\mathrm{H}_{2} \mathrm{O}\right)$ of $8.8 \times 10^{-5}$ $\mathrm{g} / \mathrm{cc}$ or less.

Acceptance Test - Testing which allows for the inference that a canister is dry. For this procedure, the acceptance test for a canister to be inferred dry is the maintenance of $<80$ torr vacuum for 30 minutes at $120^{\circ} \mathrm{F}$.

Dewatering Skid - The equipment that removes water from the TMI-2 canisters; after removal the water is passed through a filter and ion exchanger to remove suspended solids and radioactivity.

Lower Explosive Limit (LEL) - The boundary mixture (minimum concentration of the material) of a gas (hydrogen) which, if ignited, will just propagate a flame. The lower explosive limit of hydrogen at atmospheric temperature and pressure is 4 percent by volume in air. The terms "flammable limits' and "explosive limits" are interchangeable.

Radiolysis - Chemical decomposition by the action of radiation; for these tests this is determined by the measurement of hydrogen evolution from the decomposition of residual water in a canister due to the radioactive energy emitted by the contents of a canister.

Vacuum Condenser Skid - Refrigeration equipment for removing water vapor from the TMI-2 canisters off gas from the Vacuum Furnace.

Vacuum Furnace - A vessel capable of heating one to four TMI-2 canisters in a vacuum environment.

Vacuum Pump Skid - Equipment capable of developing a vacuum on (or pressurizing) a closed system (Vacuum Furnace, shielding Cask, or Dry Shielded Canister. 